


\section{APPROVAL SHEET}

Title of Dissertation: "Reversing Breast Cancer-Induced Immune Suppression"

Name of Candidate: Daniel W Beury

Doctor of Philosophy, 2015

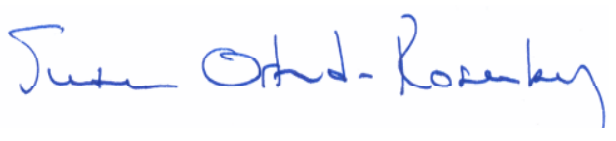

Dissertation and Abstract Approved:

Suzanne Ostrand-Rosenberg

Professor of Biological Sciences

Robert and Jane Meyerhoff

Professors of Biochemistry

Date Approved: $11 / 25 / 2015$ 


\begin{abstract}
Title of Document:

Reversing Breast Cancer-Induced Immune
Suppression

Daniel W Beury, Ph.D., 2015

Directed By:

Dr. Suzanne Ostrand-Rosenberg, Professor, Biological Sciences, Robert and Jane Meyerhoff, Professors of Biochemistry, Department of Biological Sciences

The immune system is capable of eradicating transformed cells. However, tumor cells and host cells present in the tumor secrete pro-inflammatory mediators that promote the accumulation and activity of myeloid-derived suppressor cells (MDSC), which potently suppress anti-tumor immunity. MDSC and macrophages are present in most solid tumors and it is established that cross-talk between MDSC and macrophages impacts anti-tumor immunity; however, interactions between tumor cells and MDSC or macrophages are less well studied. Using four murine tumor cell lines, we examined potential interactions between these cells in vitro and in vivo. In vitro studies demonstrated that MDSC-secreted IL-10 decreased macrophage-derived IL-6 and TNF $\alpha$, and increased nitric oxide (NO). IL-6 indirectly decreased MDSC IL-10. Tumor cells increased MDSC IL-6 and vice versa; and increased macrophage IL-6 and NO, and decreased macrophage TNF $\alpha$. Tumor-cell-driven macrophage IL-6 was reduced by
\end{abstract}


MDSC, and tumor cells and MDSC enhanced macrophage NO. In vivo studies identified that IL-6 and IL-10 were produced by stromal cells in the tumor. These results demonstrate that MDSC, macrophage, and tumor cell interactions potentially alter the inflammatory milieu within the tumor microenvironment and drive tumor growth. Release of reactive oxygen species (ROS) is one of the mechanisms used by MDSC to suppress anti-tumor immunity. Although ROS are toxic to most cells, MDSC survive despite their elevated content and release of ROS. Nuclear factor erythroid derived 2-like 2 (Nrf2) is a transcription factor that regulates a battery of genes which attenuate oxidative stress. Therefore, we hypothesized that MDSC resistance to ROS may be due to their up-regulation of Nrf2. Murine studies demonstrated that Nrf2 enhanced MDSC suppressive activity and increased the quantity of tumor-infiltrating MDSC by reducing their oxidative stress and rate of apoptosis. Nrf2 did not affect circulating levels of MDSC in tumor-bearing mice since the decreased apoptotic rate of tumor-infiltrating MDSC was balanced by a decreased rate of differentiation from bone marrow progenitor cells. These results demonstrate that Nrf2 regulates the generation, survival and suppressive potency of MDSC, and that a feedback homeostatic mechanism maintains a steady-state level of circulating MDSC in tumor-bearing individuals. 


\title{
REVERSING BREAST CANCER-INDUCED IMMUNE SUPPRESSION
}

\author{
By
}

Daniel Wirth Beury

Dissertation submitted to the Faculty of the Graduate School of the University of Maryland, Baltimore County, in partial fulfillment of the requirements for the degree of

Doctor of Philosophy 
(C) Copyright by

Daniel Wirth Beury

2015 



\section{Acknowledgments}

I would like begin by thanking my parents, Pete and Kathy Beury. You have given me unbridled emotional support, financial aid, and love throughout my life. I feel incredibly lucky to have such a strong relationship with you, and I cannot properly express how grateful I am for everything you have done for me.

To my sister Kate, thank you for lending me an ear, as well as your artistic inspirations and talents. You truly care about me and would do everything in your power to help me if I was in need. I value our friendship and look forward to growing our relationship throughout our lives.

To my significant other, Margarita, I am incredibly fortunate to have met you in my journey through graduate school. I met you at a very difficult time in my life, and you have always been completely supportive. Interacting with you has always been a bright spot in my day. I have enjoyed introducing you to life in the United States, and have appreciated being exposed to your culture. You have been a positive influence in my life and a source of constant source of encouragement. Thank you so much for the kind words and pampering while I worked on this dissertation. You have always been there when I needed you, and am looking forward to growing together and supporting you while you complete your dissertation.

To my friends, if it were not for you I would have completed this dissertation two years earlier. All joking aside, thank you very much for making me smile, providing a much needed outlet to vent frustration, and providing ample opportunities for me to relax when I needed it most. 
To my committee members, thank you for your insightful questions, critiques, and ideas throughout these years. You have provided me with guidance and insight that has been essential for completing this dissertation.

To my co-workers. Ginny, you are a wonderful person and an essential cog that keeps the lab running. Whenever I have trouble finding a reagent, you have an uncanny knack for finding exactly what I needed. Thank you very much for teaching me the essential lab techniques when I first joined the lab. I have enjoyed our conversations throughout the years and look forward to continuing them in the future. Pratima, thank you for the training and guidance. You were always the first person I would discuss new ideas with. I admire the way you develop and design experiments, and our conversations have fundamentally changed the way I view, approach, and design new projects.

Lucas and Kathy, you are both incredibly bright and driven individuals, and have made my time at UMBC very enjoyable. Lucas, you are one of the most genuine people that I know and have always been willing to go the extra mile for not only me, but everyone around you. Kathy, I have enjoyed working next to you throughout the years and developing a friendship outside the lab. I think it is a testament to your perseverance that you were able to work next to me throughout the years without being driven insane. Thank you so much for the scientific support, laughs, and friendship.

Lastly, to my mentor, Sue, thank you for your amazing mentorship and guidance. You have taught me how to think critically about every experiment I perform, document I write, and presentation I give. You are without a doubt the most influential person in my life over the last decade. You pushed me to become the scientist I am today, and for that I am forever grateful. 


\section{Table of Contents}

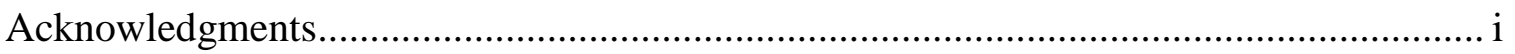

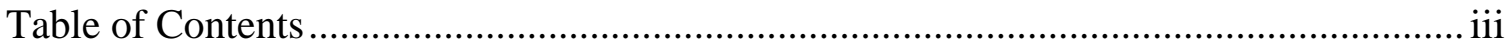

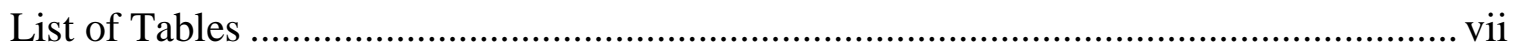

List of Figures .................................................................................................... vii

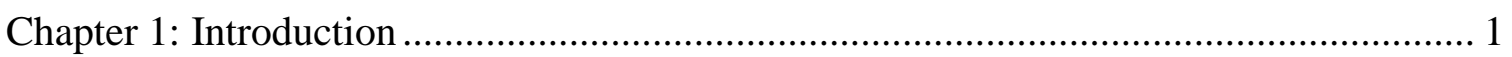

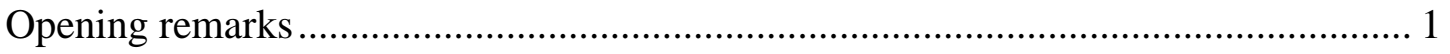

1: Cancer can be eradicated by the immune system …………………….................. 2

1.1: Immune surveillance protects us from cancer .............................................. 3

1.2: Cancer cells escape immune surveillance by immunoediting...................... 4

1.3: Significance of the preceding information to this dissertation.................... 7

2: Tumor cells express factors that suppress anti-tumor immunity and induce immunosuppressive cell populations. ............................................................. 8

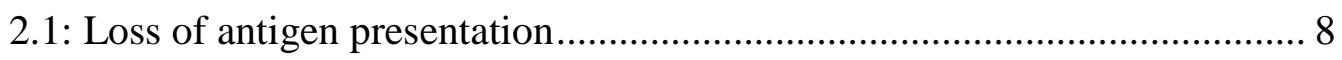

2.2: Manipulation of Immune checkpoints................................................... 9

2.3: Transforming growth factor beta (TGF $\beta)$.............................................. 10

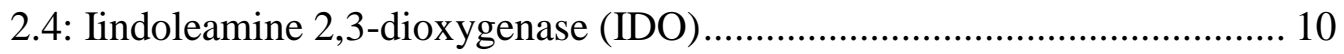

2.5: Regulatory T cells (Tregs)................................................................... 11

2.6: Immunosuppressive macrophages......................................................... 12

2.7: Myeloid-derived suppressor cells (MDSC)............................................ 13

2.8: Significance of the preceding information to this dissertation................... 13

3: Inflammation links immune suppression and cancer by inducing MDSC development and suppressive functions........................................................ 15

3.1: Vascular endothelial growth factor (VEGF) ............................................. 16

3.2: Granulocyte-macrophage colony stimulating factor (GM-CSF) and granulocyte colony stimulating factor (G-CSF) ....................................... 17

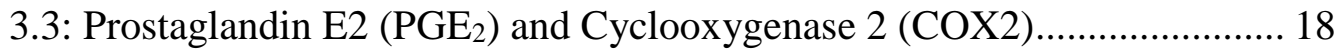

3.4: CCAAT/enhancer binding protein $\beta(\mathrm{C} / \mathrm{EBP} \beta)$ and $\mathrm{C} / \mathrm{EBP}$ homologous

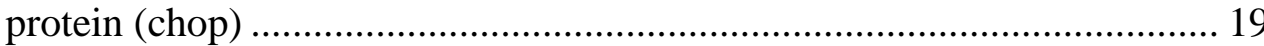

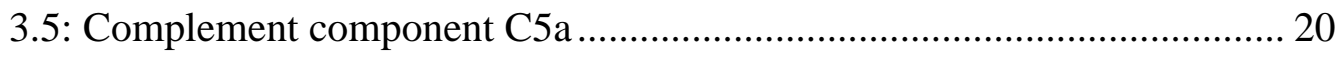

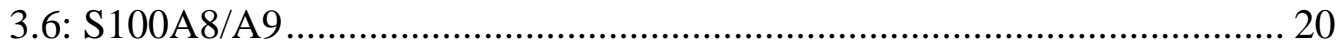

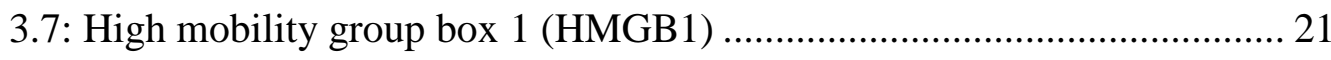

3.8: IL-1 $\beta$, IL-6, and indoleamine 2,3 dioxygenase (IDO) ............................... 21

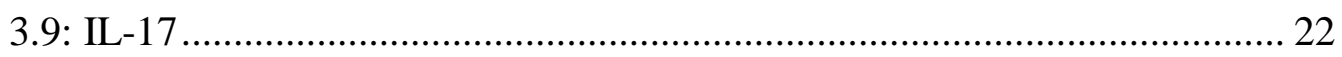

3.10: Significance of the preceding information to this dissertation................. 23

4: MDSC utilize a network of effector and signaling molecules to decrease immune surveillance. 


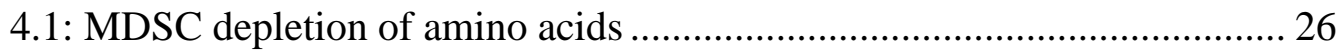

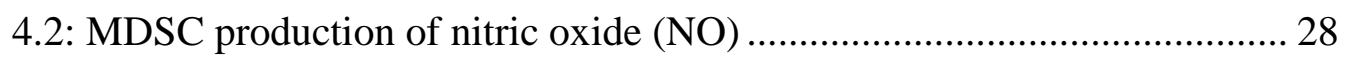

4.3: MDSC production of reactive oxygen species (ROS) ………………...... 29

4.4: MDSC inhibit $\mathrm{T}$ cell migration by down regulating L- and E- selectins. . 29

4.5: MDSC can express Programmed Death-Ligand 1 (PD-L1) ....................... 30

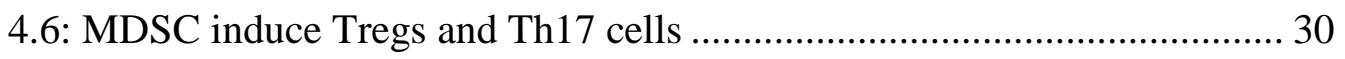

4.7: MDSC impair NK cell-mediated cytotoxicity .......................................... 31

4.8: Significance of the preceding information to this dissertation.................. 32

5: Cross-talk between MDSC, macrophages, tumor cells, and mast cells enhances

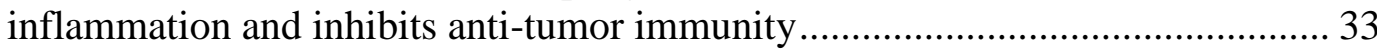

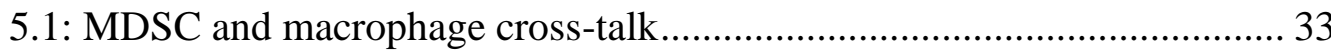

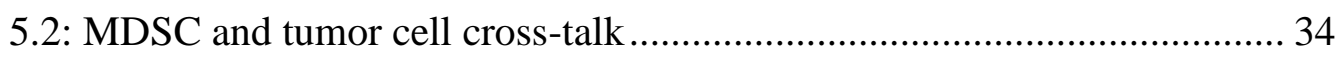

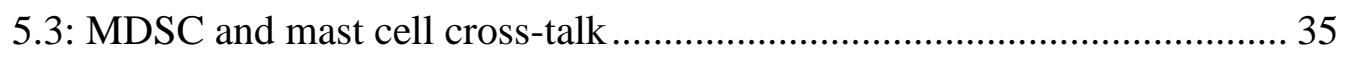

5.4: Significance of the preceding information to this dissertation................... 36

6: Nuclear factor erythroid 2 (NFE2)-related factor 2 (Nrf2) ..................................... 37

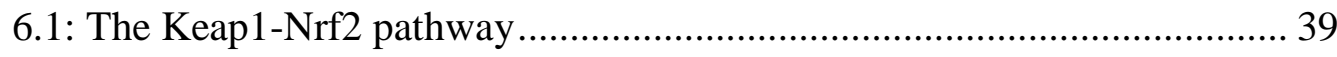

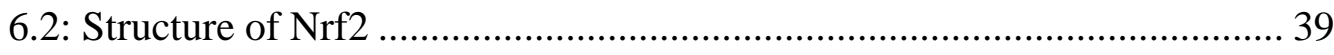

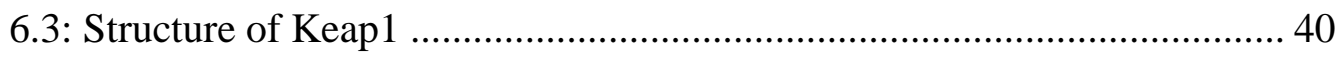

6.4: Mechanisms of Nrf2 activation ................................................................ 40

6.5: $\mathrm{Nrf} 2$ heterodimerizes with other transcription factors and mediates the

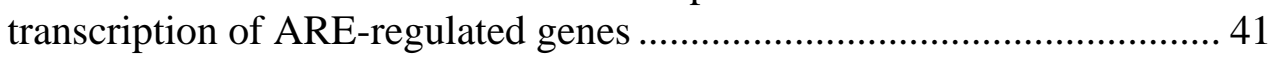

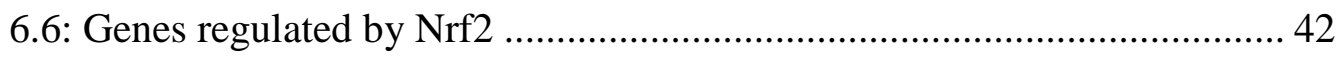

6.7 Significance of the preceding information to this dissertation.................... 42

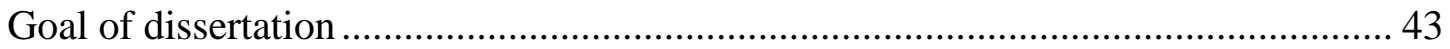

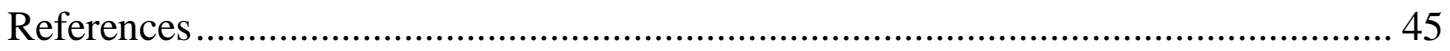

Chapter 2: Cross-talk among myeloid-derived suppressor cells, macrophages, and tumor cells impacts the inflammatory milieu of solid tumors........................................ 75

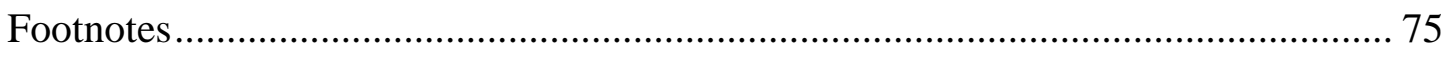

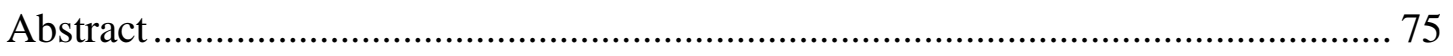

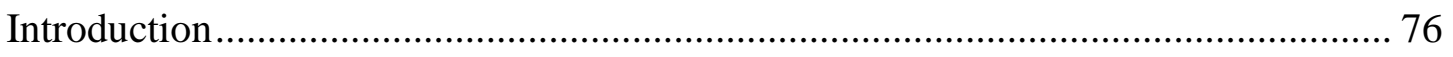

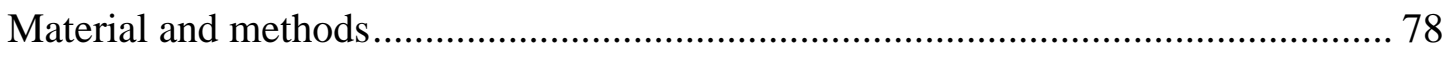

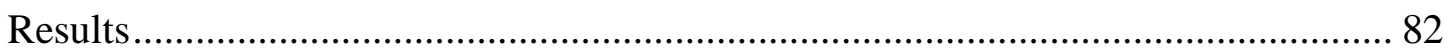

IL-6 and IL-10 promote tumor progression ................................................. 82

MDSC production of IL- 10 decreases macrophage IL- 6 and TNF $\alpha$, and increases NO. IL-6 indirectly regulates MDSC production of IL-10............... 83

Other cytokines are also impacted by interactions between MDSC and

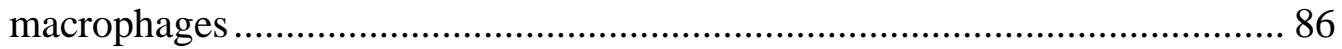

Tumor cells increase MDSC production of IL-6 and vice versa...................... 86 
Tumor cells increase macrophage IL-6 and NO, and decrease macrophage

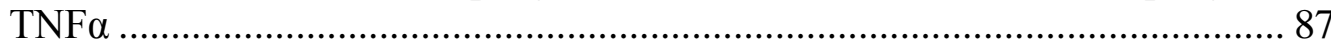

MDSC prevent most tumor cells from increasing macrophage IL-6 ............. 89

MDSC increase macrophage NO in the presence of tumor cells ...................8 89

Stromal cells are the dominant producers of IL-6 and IL-10 in the tumor

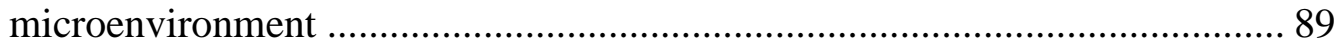

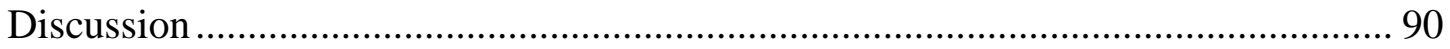

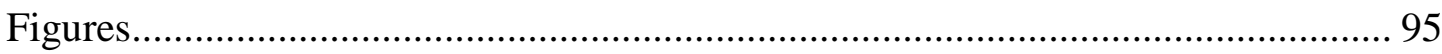

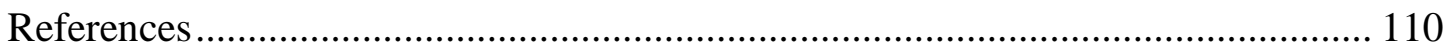

Chapter 3: Myeloid-derived suppressor cell survival and function are regulated by the transcription factor Nrf2 .................................................................. 118

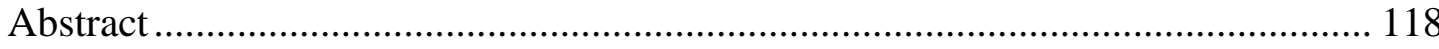

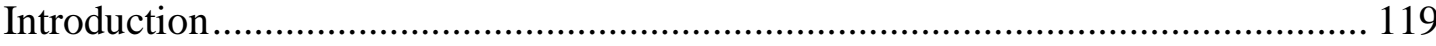

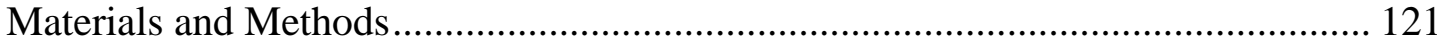

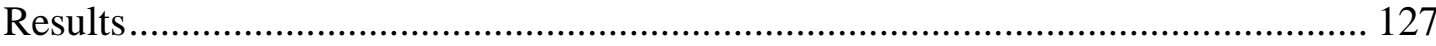

Host expression of Nrf2 enhances tumor progression.............................. 127

Host expression of Nrf2 enhances MDSC suppressive potency and the quantity of tumor-infiltrating MDSC. ..................................................................... 128

Nrf2 decreases MDSC oxidative stress and apoptosis ................................ 131

Nrf2-deficiency increases the rate of MDSC generation in the bone marrow 132

Discussion 133

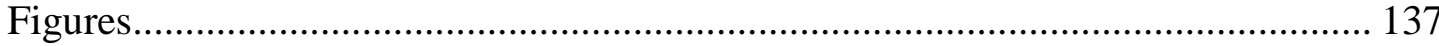

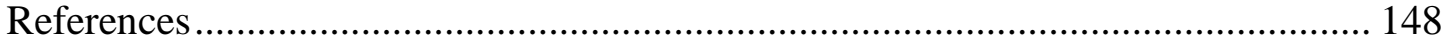

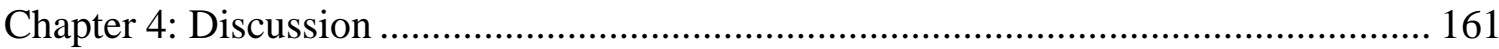

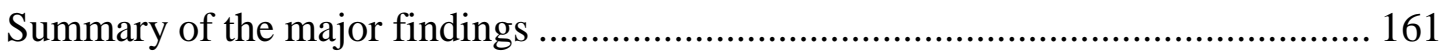

How does Nrf2 impact inflammation, tumor progression, and anti-tumor immunity? 162

How does MDSC production of IL-10 impact anti-tumor immunity? .................... 166

How does cross-talk impact other cells in the tumor microenvironment?............... 172

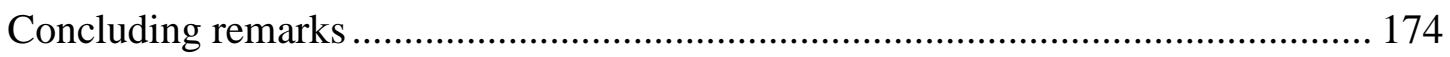

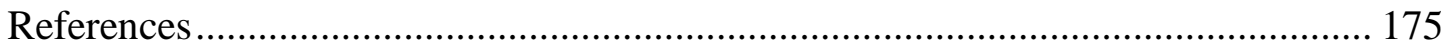

Appendix 1: Cross-talk among myeloid-derived suppressor cells, macrophages, and tumor cells impacts the inflammatory milieu of solid tumor. 
Appendix 2: IDO is a nodal pathogenic driver of lung cancer and metastasis development 202

Appendix 3: Myeloid-derived suppressor cells: critical cells driving immune suppression in the tumor microenvironment .................................................................. 217

Appendix 4: Cross-talk between myeloid-derived suppressor cells (MDSC), macrophages, and dendritic cells enhances tumor-induced immune suppression

Glossary ...... 272 


\section{List of Tables}

Chapter 2: Cross-talk between myeloid-derived suppressor cells, macrophages, and tumor cells impacts the inflammatory milieu of solid tumors

Supplemental Table 1: IL-10-deficiency and IL-6-deficiency modestly alter MDSC production of other cytokines and cross-talk with macrophages .........................108

Supplemental Table 2: NO has pro- and anti-tumor effects ..................................109 


\section{List of Figures}

Chapter1: Introduction

Figure 1: The concept of cancer immunoediting

Figure 2: Myeloid cell differentiation under normal and inflammatory conditions ....14

Figure 3: MDSC suppress T cells and regulate the inflammatory milieu by multiple mechanisms

Figure 4: Mechanism for activation of the Keap1-Nrf2 pathway .38

Chapter 2: Cross-talk between myeloid-derived suppressor cells, macrophages, and tumor cells impacts the inflammatory milieu of solid tumors

Figure 1: IL-6 and IL-10 produced by host cells enhance primary tumor growth and decrease survival time

Figure 2: Cross-talk between MDSC and macrophages regulates production of IL-10,

IL-6 and nitric oxide.

Figure 3: Tumor cells induce MDSC to produce IL-6 and vice versa.

Figure 4: Tumor cells induce macrophages to produce IL-6 and NO, but decrease macrophage TNF $\alpha$

Figure 5: MDSC decrease tumor cell-mediated enhancement of IL-6, and increase tumor cell-mediated enhancement of macrophage NO

Figure 6: Host cells are the dominant producers of IL-6 and IL-10 in the tumor microenvironment

Figure 7: Summary of cross-talk between MDSC, macrophages, and tumor cells ...105

Supplemental Figure 1A: Tumor progression, survival time, and engraftment are similar in BALB/c wild type and IL- $10^{-/-}$mice inoculated with $1 \times 10^{5}$ or $1 \times 10^{4}$ CT26 tumor cells.

Supplemental Figure 1B: Exogenous IL-6 does not impact MDSC production of IL10.

Supplemental Figure 1C: MDSC, but not tumor cells or macrophages, proliferate during co-culture

Supplemental Figure 2: Tumor cells do not affect macrophage and/or MDSC production of IL-12 or IL-10, and MDSC do not affect tumor cell and macrophage production of TNF $\alpha$. 
Chapter 3: Myeloid-derived suppressor cell survival and function are regulated by the transcription factor $\mathrm{Nrf} 2$

Figure 1: Nrf2 decreases survival time of tumor-bearing mice

Figure 2: Nrf2 enhances MDSC suppressive activity and the quantity of tumor-

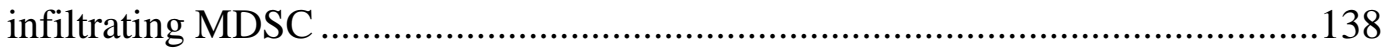

Figure 3: Nrf2 decreases intracellular MDSC oxidative stress ..............................140

Figure 4: Nrf2 protects MDSC from apoptosis........................................................141

Figure 5: Nrf2 deficiency enhances MDSC proliferation........................................142

Supplemental Figure 1: MDSC from Nrf2 ${ }^{-/}$mice are deficient for Nrf2 and do not upregulate Nrf2-regulated genes in response to stress.......................................144

Supplemental Figure 2: Nrf2 enhances MDSC-mediated suppression of T cells .....145

Supplemental Figure 3: Nrf2 does not impact MDSC production of NO, expression of Arg1 or xCT, or the down-regulation of L-selectin, but decreases MDSC production of IL-10

Supplemental Figure 4: Nrf2 does not alter the ratio of tumor-infiltrating PMNMDSC to M-MDSC or MDSC chemotaxis 


\section{Chapter 1: Introduction}

\section{Opening remarks}

In this thesis, I will discuss the research I have conducted and give an interpretation and explanation of the results. This dissertation is focused on obtaining a greater understanding of myeloid-derived suppressor cells (MDSC), an immature myeloid cell population that is induced by inflammation and cancer. MDSC are an important cell population in cancer patients because they suppress anti-tumor immunity through several mechanisms. Specifically, my work is focused on two separate aspects of MDSC: (i) how cross-talk between MDSC, macrophages, and tumor cells modulates the level of inflammatory mediators in the tumor microenvironment; and (ii) how MDSC induction, survival, and suppressive activity is regulated by the transcription factor nuclear factor (erythroid-derived 2)-like 2 (Nrf2). As such, this introduction will describe the relationship between the immune system and cancer, and how cancer escapes antitumor immunity though the activity of MDSC. This background will provide the reader with a general understanding of anti-tumor immunity, and provide sufficient background on MDSC in order to understand how this research fits into, and contributes to the global understanding of MDSC function and suppressive activity. The goal of this research was to strengthen the breadth of knowledge associated with MDSC in order to provide new insight for the development of future immunotherapies. 


\section{1: Cancer can be eradicated by the immune system}

Cancer is the second leading cause of death in the United States. It has been estimated by the American Cancer Society that in the United States in 2015, there will be over 1.6 million newly diagnosed cancer patients, and 589,000 deaths will be due to cancer (1). Most patients are treated by a combination of surgery, radiotherapy, and/ or chemotherapy. While standard therapies are efficient at treating the primary tumor, cancer is still the cause of roughly $25 \%$ of deaths in the United States. The primary reason for cancer-related deaths is because of metastatic spread of the disease, which is responsible for $90 \%$ of cancer deaths (2).

Utilization of the immune system to systematically target and destroy cancer is a promising approach for the treatment of metastatic cancer. T cells are capable of becoming activated against tumor specific antigens and tumor associated antigens, and can destroy tumor cells expressing those antigens. Antigen-specific stimulation is typically mediated by professional antigen presenting cells (APC) such as dendritic cells (DC) and macrophages. Classically, APC phagocytose and process tumor-derived proteins into short peptide sequences, and display those peptides on their surface bound to histocompatibility complex (MHC) proteins. Each T cell expresses a unique T cell receptor (TCR) that has specific reactivity toward a unique peptide bound to MHC. Helper $\mathrm{CD}^{+} \mathrm{T}$ cells are activated by peptides bound to MHC II, which is expressed by professional APC, while cytotoxic $\mathrm{CD}^{+} \mathrm{T}$ cells are activated by peptides bound to $\mathrm{MHC}$ I, which is expressed by most nucleated cells $(3,4)$. Activation of $T$ cells against tumor antigens can potentially establish long-term immunologic memory which can target latent 
metastasis. Activated $\mathrm{CD}^{+} \mathrm{T}$ cells mediate direct killing of tumor cells, while $\mathrm{CD}^{+}{ }^{+} \mathrm{T}$ cell activation results in the release of cytokines such as INF $\gamma$ and IL-2 $(5,6)$, which can further enhance anti-tumor immunity by promoting the activation of $\mathrm{T}$ cells in a positivefeedback manner, enhance MHC expression and antigen presentation on APC, increase direct tumor cell killing by macrophages and natural killer (NK) cells $(7,8)$, and induce the switching of B lymphocytes (B cells) to antibody-producing plasma B cells which can have long-term anti-tumor effects $(6,9)$.

\section{1: Immune surveillance protects us from cancer}

The idea that the ever-vigilant immune system is continually detecting and eradicating transformed cells from our bodies was originally proposed by Paul Ehrlich in 1909 (10), and the hypothesis of immune surveillance was further elaborated by Burnet and Thomas in 1957 (11). Indeed, athymic nude mice have severely diminished levels of T lymphocytes and display enhanced susceptibility to methylcholanthrene-induced sarcomas (12). Similar results were observed in recombination activating gene 2 (RAG-2) deficient mice, which cannot rearrange T nor B cell receptors and lack lymphocytes (13). Additional studies focusing on disruption of TCR signaling in T and natural killer T (NKT) cells (14), and the synthesis of $\operatorname{IFN} \gamma(15,16)$ and perforin $(17)$ in the regulation of tumor development highlight the necessity of lymphocytes for effective immune surveillance. 


\section{2: Cancer cells escape immune surveillance by immunoediting}

Despite the notion that our immune system is diligently protecting us from precancerous cells, some cells are able to evade detection and destruction by the immune system and form nascent malignancies (18). This process gave rise to the concept of immunoediting which consists of three phases: elimination, equilibrium, and escape (Figure 1) (19). Healthy cells can become transformed due to genome instability in the form of mutations, gene amplification, or chromosome rearrangements, which can cause oncogene activation, tumor suppressor deactivation, or the dysregulation of cell cycle genes (20). Transformed cells are typically highly immunogenic and frequently express tumor-specific and tumor-associated antigens, NK cell ligands, and damage-associated molecular pattern molecules (DAMPs) which facilitate an anti-tumor immune response that destroys these transformed cells during the elimination phase (19). However, some tumor cell populations are able to escape immediate destruction by the immune system. These populations can exist in equilibrium with the immune system as continued outgrowth of the tumor cell population is held in check by the immune system. The equilibrium phase is the longest stage of immunoediting; where tumor cell populations can become dormant, or tumor cell variants can acquire additional mutations that confer a selective advantage for continued growth. These advantages arise as an adaptation to selective pressures exerted by the immune system, which selects for immune-evasive and/or immunosuppressive tumor cell variants $(19,21)$. The escape phase represents the outgrowth of tumor cells that evade destruction by the immune system. The escape phase can also result from the establishment of MDSC, which are the primary focus of this 
dissertation. Other immunosuppressive cell mechanisms and cell populations can also facilitate immune escape and are further discussed in section 2. 


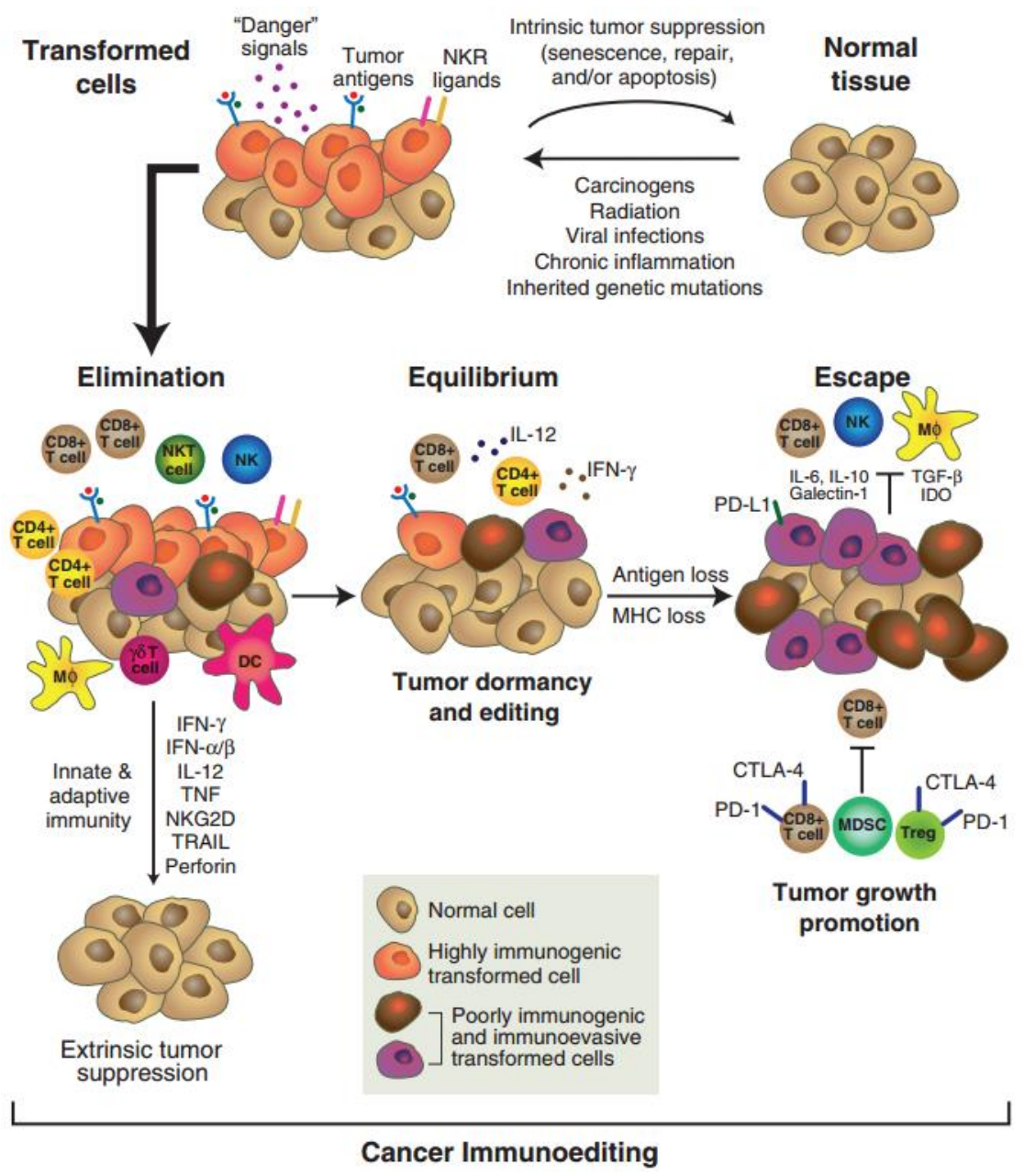

Figure 1: The concept of cancer immunoediting. Normal tissue can become transformed through somatic mutations that dysregulate the cell cycle. Mutations can be inherited, or be caused by exposure to carcinogens, radiation, inherited genetic defects, or chronic inflammation. Transformed cells release danger signals and express tumor antigens that designate themselves as tumor cells. During the elimination phase, these danger signals and tumor antigens are utilized by the immune system to target and destroy the transformed cells. However, random mutations can allow a tumor cell to become immune evasive which allows the transformed cells to enter the equilibrium phase. Due to selective pressure from the immune system, immune evasive and immune suppressive tumor cells are able to emerge out of the equilibrium phase and in into the escape phase. These immune evasive and immune suppressive tumor cells escape detection and destruction from the immune system by losing antigen presentation, express immunosuppressive molecules such as transforming growth factor beta (TGF $\beta$ ), indoleamine 2,3-dioxygenase (IDO), and programed death ligand 1 (PD-L1), and induce immunosuppressive cell populations such as T regulatory cells (Treg) and myeloidderived suppressor cells (MDSC). Figure adapted from $(19,22)$. 


\section{3: Significance of the preceding information to this dissertation}

The immune system is capable of destroying malignant cells. However, selective pressures exerted by the immune system on tumor cells selects for tumor cell variants that escape destruction by the immune system. One of the mechanisms that tumors use to facilitate immune escape is by inducing MDSC. Therefore, understanding mechanisms by which the immune system is capable of targeting and destroying malignant cells is essential for the reader to appreciate the negative effects MDSC have on anti-tumor immunity. 


\section{2: Tumor cells express factors that suppress anti-tumor immunity and induce immunosuppressive cell populations.}

As briefly discussed in the earlier section, tumor cells are capable of evading destruction by the immune system. Tumor-induced immune suppression is a major problem for cancer patients, and deficiencies in immune responses have been extensively characterized in tumor-bearing patients and animals. T cells become tolerant to tumor antigens, and tumor infiltrating lymphocytes and APC in tumor tissue are nonfunctional (23). These defects in the immune system occur through multiple mechanisms that are overlapping and redundant. Tumor cells can avoid antigen presentation, or directly suppress T cell activation by expressing programmed death ligand-1 (PD-L1), TGF $\beta$, or indoleamine 2,3-dioxygenase (IDO). Tumor cells can also induce immunosuppressive cell types such as regulatory T cells (Tregs), M2 macrophages, and MDSC (23, 24).

\section{1: Loss of antigen presentation}

Tumors frequently evade destruction by cytotoxic $\mathrm{CD}^{+} \mathrm{T}$ cells by downregulating antigen presentation machinery. $\mathrm{CD}^{+} \mathrm{T}$ cells can recognize tumor antigens bound to MHC I expressed on tumor cells, which induces $\mathrm{CD}^{+} \mathrm{T}$ cell-mediated killing of the tumor cell. Thus, decreased expression of MHC I, or the intracellular machinery involved in antigen presentation can help tumor cells avoid recognition by the immune system $(25,26)$. 


\section{2: Manipulation of Immune checkpoints}

For $\mathrm{T}$ cells to be activated against tumor antigens and be effective in an antitumor immune response, two signals are required for activation. The first signal is initiated by the binding of the TCR to a specific peptide-MHC complex, which induces a

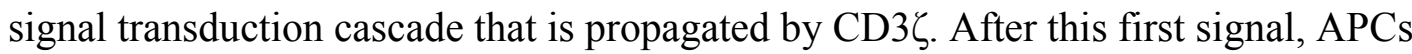
deliver a second, co-stimulatory signal which is essential for enhancing the survival and inducing the clonal expansion of activated T cells. One of the most studied forms of costimulation is via CD80 or CD86 binding to the co-stimulatory receptor CD28 on T cells (27). However, co-inhibitory molecules exist to quench immune activity and restore homeostasis (28). Cytotoxic T lymphocyte antigen-4 (CTLA4) is a well-characterized coinhibitory molecule $(29,30)$, which is expressed at high levels on activated $\mathrm{T}$ cells and $\mathrm{T}$ regulatory cells (Tregs). CTLA4 binds to CD80/CD86 with a higher affinity than CD28, which results in decreased IL-2R expression and IL-2 production by the T cell, and arrests the $\mathrm{T}$ cell in the G1 phase of the cell cycle (29). Additionally, CTLA4 can remove CD80/CD86 from APC through trans-endocytosis, which presumably results in less available CD80/CD86 to stimulate CD28 (31). Since tumor cells can induce Tregs which express CTL4 (discussed in section 2.5), CTLA4 is an important obstacle in anti-tumor immunity.

Another well characterized immune checkpoint molecule is the programmed death 1 (PD-1) pathway. Like CTLA4, PD-1 is expressed on activated T cells (28). PD-1 induces apoptosis in $\mathrm{T}$ cells when it is bound by programmed death ligand 1 or 2 (i.e. PDL1 and PD-L2, respectively) (28). Many tumor cells constitutively express or are induced by IFN $\gamma$ to express PD-L1 $(32,33)$, which is known to be an important protective 
mechanism that facilitates tumor growth (34). PD-L2 can also be expressed on tumor cells, however, PD-L2 expression in human malignancies has not been correlated with decreased survival outcome with statistical significance (35).

\section{3: Transforming growth factor beta (TGF $\beta)$}

TGF $\beta$ is another important molecule that is secreted by tumor cells or other host immune cells that promotes tumor progression and decreases anti-tumor immunity (36). TGF $\beta$ negatively impacts the cytolytic activity of $\mathrm{CD}^{+} \mathrm{T}$ cells and decreases their expression of perforin, granzyme A and B, Fas-ligand, and IFN $\gamma$ (37). TGF $\beta$ also induces immunosuppressive cell populations such as Tregs and M2 macropahges which are discussed in sections 2.5 and 2.6, respectively.

\section{4: Indoleamine 2,3-dioxygenase (IDO)}

Following initial contact with antigen, $\mathrm{T}$ cells undergo metabolic changes that are essential for their activation and clonal expansion. Limitation of essential amino acids such as L-Tryptophan (L-Trp) can limit T cell responses. During amino acid starvation, accumulation of empty aminoacyl tRNAs occurs, which activates serine-threonine kinase GCN2. GCN2 phosphorylates eIF2 $\alpha$, which binds eIF2B and suppresses the translation initiation complex from binding charged aminoacyl tRNA, thereby causing a global decrease in protein translation. Simultaneously, GCN2 enhances the translation of GCN4, which results in the transcription of genes required for the synthesis of amino acids (38). L-Trp metabolism by IDO, which degrades L-Trp into N-formylkynurenine, is an 
important regulatory mechanism for APC to modulate $\mathrm{T}$ cell functions during antigen presentation (39). Tumor cells and other immunosuppressive cells utilize this pathway to inhibit $\mathrm{T}$ cell activation and proliferation (40).

\section{5: Regulatory T cells (Tregs)}

Tregs naturally occur in the thymus and are an important $\mathrm{CD} 4^{+}$helper $\mathrm{T}$ cell subset that helps maintain tolerance to self-antigens and subvert autoimmunity (41). They are defined by the surface markers CD4 and CD25 (IL-2R $\alpha$ ), and expression of the transcription factor FoxP3 (42). Tregs are induced in response to the inflammatory mediators IL-10, TGF $\beta$, and $\mathrm{PGE}_{2}$, and multiple studies have demonstrated that Tregs are a barrier for anti-tumor immune responses (43). Tregs suppress $\mathrm{T}$ cell activation by several mechanisms: (i) induce apoptosis in $\mathrm{B}$ cells, $\mathrm{NK}$ cells, and $\mathrm{CD} 8^{+} \mathrm{T}$ cells in a granzyme and perforin-dependent manner $(44,45)$, and in $\mathrm{CD}^{+} \mathrm{T}$ cells via the TRAILDR5 (tumor-necrosis factor-related apoptosis-death receptor 5) pathway (46); (ii) induce DC to produce IDO (39); (iii) express CTLA4 (47), which removes CD80/CD86 from APC through trans-endocytosis (31); (iv) secrete immunosuppressive cytokines such as IL-10, IL-35, and TGF $\beta$ (42), and; (v) express high levels of the IL-2 receptor, which depletes the local environment of IL-2 available for effector T cells (42). Tregs are also induced by MDSC, which is discussed in section 5.6. 


\section{6: Immunosuppressive macrophages}

Macrophages are phagocytic, tissue-resident myeloid cells that are derived from circulating monocytes. They can exhibit a continuum of phenotypes and functions which can promote or deter tumor growth. Their status is determined by the stimuli in their microenvironment. M1-like macrophages are anti-tumor and are induced by IFN $\gamma$ and bacterial products such as LPS. They express high levels of reactive oxygen species, are IL-12 $2^{\text {high }}$ IL-6 ${ }^{\text {high }}$ TNF $\alpha^{\text {high }}$ MHCII $^{\text {high }}$ CD86 ${ }^{\text {high }}$ IL-10 ${ }^{\text {low/neg }}$ NOS2 ${ }^{\text {high }}$ ARG $1^{\text {low }}$, kill tumors by secreting NO, and are efficient activators of type 1 anti-tumor immune responses. M2like macrophages are pro-tumor and are induced by IL-4, IL-13, TGF $\beta$, and IL-10, are

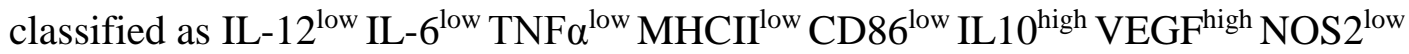
$\mathrm{ARG} 1^{\text {high }}$, and are responsible for tissue remodeling and angiogenesis in addition to their pro-tumor activity (48). Macrophages can suppress T cell activation by expressing PD-L1 and CTLA4, induce Tregs by secreting IL-10 and TGF $\beta$, and deplete the local environment of L-arginine through the activity of nitric oxide synthase (NOS) and arginase 1 (ARG1) (49). NOS and ARG1 are also utilized by MDSC to suppress T cell activation and proliferation, and are further discussed in sections 4.1 and 4.2. Cross-talk between macrophages and MDSC exacerbates the immune-suppressive, pro-tumor activity of each of these cell populations $(8,50,51)$. Understanding the role of cross-talk in regulating MDSC and macrophage activity was one of the goals of this dissertation and is introduced in section 5.1, and the results obtained during this dissertation are discussed in chapter 2. 


\section{7: Myeloid-derived suppressor cells (MDSC)}

MDSC are a heterogeneous population of immature myeloid cells that are induced by inflammation, and are widely accepted as one of the main obstacles for anti-tumor immune responses. MDSC are derived from common myeloid progenitor cells in the bone marrow, which also gives rise to other myeloid cells such as macrophages, dendritic cells, and neutrophils (Figure 2). MDSC are identified by the surface markers Gr1 and CD11b in mice, and CD14, CD15, CD33, and CD11b in humans (52-54). They are largely grouped into two distinct phenotypes, monocytic (M-MDSC) and granulocytic (PMN-MDSC). In mice, Gr1 exists as two isoforms, Ly6G and Ly6C. M-MDSC are

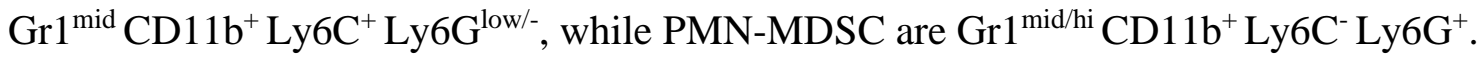
Since MDSC and neutrophils have similar morphology and surface markers, the defining characteristic of bona fide MDSC is their ability to suppress $\mathrm{T}$ cell function. The numerous suppressive mechanisms that MDSC use to inhibit anti-tumor immunity are discussed in sections 4 and 5 .

\section{8: Significance of the preceding information to this dissertation}

Tumors employ multiple mechanisms to escape destruction by the immune system. Understanding the mechanisms that tumors utilize to subvert anti-tumor immunity allows the reader to appreciate how MDSC fit into the context of tumorinduced immune suppression. 


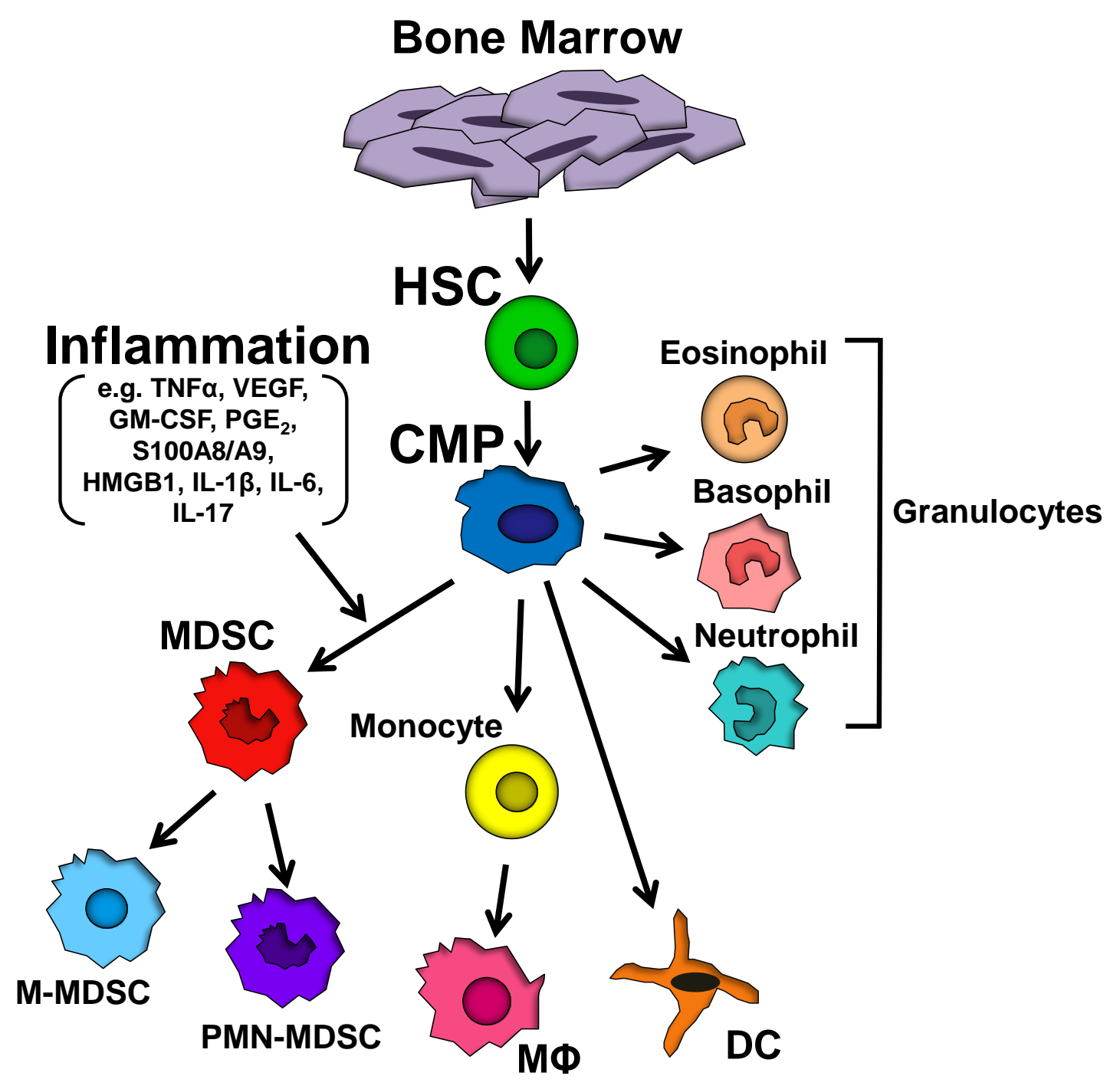

Figure 2: Myeloid cell differentiation under normal and inflammatory conditions. Myeloid cells originate from bone marrow-derived hematopoietic stem cells (HSCs) that differentiate into common myeloid progenitors (CMPs). During normal myelopoiesis, CMPs differentiate into granulocytes including eosinophils, basophils, and neutrophils, as well as monocytes, macrophages, and dendritic cells. MDSCs also differentiate from CMPs and are categorized as M-MDSCs or PMN-MDSCs. Inflammation perturbs normal myelopoiesis and drives CMPs to differentiate into MDSC. HSC, hematopoietic stem cell; CMP, common myeloid progenitor; DC, dendritic cell; MФ, macrophage; MMDSCs, monocytic myeloid-derived suppressor cells; PMN-MDSCs, granulocytic myeloid-derived suppressor cells. Figure adapted from (55). 


\section{3: Inflammation links immune suppression and cancer by inducing MDSC development and suppressive functions}

Studies evaluating patients on long-term use of nonsteroidal anti-inflammatory drugs (NSAIDS), epidemiological analyses, and trials involving blockade of inflammatory molecules have demonstrated that inflammation contributes to the onset of cancer (56). Inflammation contributes to tumor progression by supplying growth factors that sustain tumor cell proliferation, survival factors that limit tumor cell apoptosis, proangiogenic factors and extracellular matrix-modifying enzymes that facilitate tumor angiogenesis, invasion, and metastasis, and factors that promote the epithelialmesenchymal transition of tumor cells $(24,57-59)$. In addition, inflammation induces immune cells to release reactive oxygen species (ROS), which damage DNA and are mutagenic, thereby accelerating tumor cell acquisition of characteristics that facilitate tumor cell escape from the immune system via immunoediting (58).

Four main sources of inflammation promote carcinogenesis: (i) environmental inflammation such as tobacco smoke which is associated with lung cancer (60); (ii) therapy-induced inflammation (e.g. from radiation and chemotherapy) that causes necrosis of tumor and tumor stromal cells, which initiates an inflammatory response similar to wound healing and may enhance presentation of tumor antigens, but may also create tumor-promoting inflammation $(61,62)$; (iii) chronic inflammation or infection which increases the risk of developing cancer (e.g. patients with Crohn's disease have increased risk of developing small bowel, colorectal, or extra-intestinal cancer, and lymphoma, while patients infected with hepatitis B or C, Schistosoma, or Bacterocides 
have a higher incidence of developing hepatocellular carcinoma, bladder cancer, and colon cancer, respectively) (63-66), and; (vi) tumor-associated inflammation, which is discussed in further detail below.

Solid tumors are a complex and frequently inflamed microenvironment. The inflammation is driven by pro-inflammatory mediators which are secreted by tumor cells, various tumor-infiltrating T and B lymphocytes, NK cells, tumor-associated fibroblasts, and myeloid cells such as neutrophils, eosinophils, mast cells, macrophages, dendritic cells, and myeloid-derived suppressor cells (MDSC) (67-69). These cells produce a vast array of inflammatory mediators that include cytokines (e.g. IL-1, IL-6, IL-17, TNF $\alpha$ ), growth factors (e.g. TGF $\beta$, GM-CSF, VEGF), and other effector molecules (e.g. S100A8/A9, High Mobility Group Box 1), which differentially impact MDSC accumulation and their suppressive functions $(8,55,70)$. Since the tumor microenvironment varies between tumor types and individuals with cancer, as well as with stage of tumor progression, it is not surprising that MDSC are a heterogeneous population that may vary from individual to individual. The specific effects of individual inflammatory mediators on MDSC are discussed below.

\section{1: Vascular endothelial growth factor (VEGF)}

VEGF is a pro-inflammatory growth factor that stimulates angiogenesis, and tumors producing high levels of VEGF have a poor prognosis. VEGF inhibits NF-kB activation which blocks DC development while simultaneously driving MDSC accumulation (71). MDSC express the VEGF receptor, and VEGF is a chemoattractant for MDSC. ROS production by MDSC increases oxidative stress which upregulates 
MDSC expression of the VEGF receptor (72). Since other factors in solid tumors also contribute to oxidative stress, the tumor microenvironment is a critical factor in determining the responsiveness of MDSC to VEGF.

In addition to tumor cells, MDSC themselves produce VEGF, thereby creating an autocrine feed-back loop that sustains MDSC accumulation (73). VEGF has been shown to be released from the extracellular matrix by MMP9, a matrix degrading enzyme (74). Soluble MMP9 is produced by tumor cells and promotes MDSC accumulation and tumor angiogenesis (75). Therefore, MDSC have multiple modes of generating VEGF.

\section{2: Granulocyte-macrophage colony stimulating factor (GM-CSF) and granulocyte colony stimulating factor $(G-C S F)$}

GM-CSF is a growth factor for leukocytes. It is required for DC differentiation and is used to expand DC ex vivo. GM-CSF induces MDSC accumulation in vivo and in vitro, while in vivo knockdown of GM-CSF reduces MDSC expansion $(76,77)$. Inclusion of GM-CSF in cultures of bone marrow progenitor cells drives the differentiation of MDSC, demonstrating that GM-CSF is a growth factor for MDSC (78).

MDSC differentiation is also positively regulated by the growth factor G-CSF. GCSF plays a critical role in mobilizing bone marrow stem cells and is essential for differentiation of granulocytic lineages (79). Administration of G-CSF to tumor-bearing mice drives tumor growth and angiogenesis, while blockade of G-CSF reduces MDSC levels (80). G-CSF also pre-conditions metastatic sites by mobilizing MDSC (81). While the role of G-CSF in MDSC development is clear, the impact of G-CSF on MDSC function is more complicated. In mice bearing MCA203 sarcomas, G-CSF induced 
$\mathrm{Gr} 1{ }^{\text {hi }} \mathrm{CD} 11 \mathrm{~b}^{+}$cells that were less suppressive than $\mathrm{Gr} 1{ }^{\mathrm{int}} \mathrm{CD} 11 \mathrm{~b}^{+}$cells, while in MMTVPyMT transgenic mice with mammary carcinoma, G-CSF caused CD11b ${ }^{+} \mathrm{Ly}_{6} \mathrm{G}^{+} \mathrm{Ly} 6 \mathrm{C}^{+}$ cells to secret BV8. BV8 is an endocrine analogue of VEGF and functions as a proangiogenic protein that promotes hematopoiesis $(81,82)$. Therefore, G-CSF differentially affects MDSC function depending on the type of tumor.

\section{3: Prostaglandin E2 $\left(\mathrm{PGE}_{2}\right)$ and Cyclooxygenase 2 (COX2)}

$\mathrm{PGE}_{2}$ is a potent inflammatory mediator that is generated by COX2-mediated conversion of arachidonic acid. $\mathrm{PGE}_{2}$ supports tumor growth by promoting angiogenesis, stimulating tumor cell proliferation, protecting tumor cells from apoptosis, and by suppressing innate and adaptive immunity. Many human and mouse tumors as well as tumor-infiltrating cells produce $\mathrm{COX} 2$ and $\mathrm{PGE}_{2}$. $\mathrm{PGE}_{2}$ promotes MDSC differentiation at the expense of $\mathrm{DC}$, while inhibition of $\mathrm{COX} 2$ or $\mathrm{PGE}_{2}$ in tumor-bearing mice blocks MDSC differentiation and delays tumor progression $(83,84)$. In the tumor microenvironment $\mathrm{PGE}_{2}$ mediates its effects through four integral membrane G-protein coupled prostanoid receptors: EP1, EP2, EP3, and EP4. Mice deficient in EP2 display delayed tumor progression and reduced MDSC levels (83). Blockade of $\mathrm{PGE}_{2}$ or EP4 in tumor-bearing mice reduces MDSC production of ARG1 (85). In human blood progenitor cells, $\mathrm{PGE}_{2}$ promotes the propagation of $\mathrm{MDSC}\left(\mathrm{CD} 11 \mathrm{~b}^{+} \mathrm{CD} 33^{+}\right.$cells $)$that have elevated levels of NOS2, ARG1, IL-10, and IL-4R $\alpha$ (86). Therefore, in mouse and human MDSC, $\mathrm{PGE}_{2}$ not only regulates the differentiation of MDSC, but several suppressive mechanisms as well. 


\section{4: CCAAT/enhancer binding protein $\beta(C / E B P \beta)$ and $C / E B P$ homologous protein}

(chop)

C/EBP proteins are a family of leucine zipper transcription factors that regulate inflammation and myeloid cell differentiation. While there are various isoforms of C/EBP proteins, $\mathrm{C} / \mathrm{EBP} \beta$ acts during stress/inflammation-induced myelopoiesis. C/EBP $\beta$ has three isoforms: LAP* and LAP (liver-enriched activator proteins), and LIP (liverenriched inhibitory protein). LAP* and LIP are transcriptional activators that drive inflammatory myelopoiesis by inducing IL-6 and ARG1. In contrast, LIP inhibits LAP signaling promoting an anti-inflammatory response. In inflammatory settings such as the tumor microenvironment, LAP* and LAP are active and drive inflammation-induced myelopoiesis. $\mathrm{C} / \mathrm{EBP} \beta$ is also required for the ex vivo generation of immunosuppressive MDSC from bone marrow progenitor cells, via IL-6 and GM-CSF (87).

$\mathrm{C} / \mathrm{EBP}$ homologous protein (chop) regulates $\mathrm{C} / \mathrm{EBP} \beta$ expression, and is part of an integrated stress response system that is activated by ROS. Therefore, tumor-induced MDSC have increased chop expression (88). MDSC from chop-deficient tumor-bearing mice are less suppressive and exhibit reduced signaling through $\mathrm{C} / \mathrm{EBP} \beta$, which results in lower expression of IL-6 and phosphorylated STAT3. Over-expression of IL-6 in chopdeficient mice rescues MDSC suppressive activity (88). Therefore, chop regulates C/EBP $\beta$ which induces IL-6, activates STAT3, and drives the suppressive potency of MDSC. 


\section{5: Complement component $C 5$ a}

C5a (also known as anaphylatoxin) is a pro-inflammatory member of the complement and lectin pathway. When the complement pathway is activated, C5a in the blood becomes fixed in tissues. C5a triggers degranulation of mast cells, aids in vascular permeability, and stimulates smooth muscle contraction. In a tumor setting C5a increases MDSC-mediated immune suppression by chemoattracting C5a receptor ${ }^{+}$MDSC to tumor vasculature, and by increasing MDSC production of ROS and ARG1 (89).

\section{6: $S 100 A 8 / A 9$}

S100A8/A9 proteins are pro-inflammatory danger signals. They are calcium binding proteins that are localized in the cytoplasm or nucleus of myeloid cells, and are released in response to cell damage, infection, or inflammation. Mice deficient in S100A9 reject transplanted tumors, while elevated expression of S100A8/A9 in solid tumors perpetuates inflammation by chemoattracting leukocytes that produce additional inflammatory molecules $(90,91)$. MDSC are one of the leukocyte populations that are chemoattracted by S100A8/A9, and chemoattraction is dependent on signaling through receptor for advanced glycation endproducts (RAGE) (91). S100A8/A9 mediate their pro-inflammatory effects by binding to the plasma membrane receptors TLR4, carboxylated N-glycans, RAGE, or heparin sulfate (92). MDSC amplify their own

accumulation by secreting S100A8/A9, thus creating a self-sustained feedback loop (91). 


\section{7: High mobility group box 1 (HMGB1)}

HMGB1 is the second most abundant protein within a cell and is released from myeloid cells as a danger response to sepsis, infection, or arthritis. HMGB1 can signal through a number of receptors including thrombospondin, CD24, TLR2, 4, 7 and 9, as well as RAGE (93). HMGB1 is required for the differentiation of MDSC. Additionally, HMGB1 modulated MDSC-mediated down-regulation of T cell L-selectin (CD62L) via up-regulation of MDSC expression of extracellular A Disintegrin And Metalloproteinase 17 (ADAM17), a protease that cleaves L-selectin (94). Secretion of the pro-tumor cytokines IL-10 and IL-1 $\beta$ by MDSC is also increased by HMGB1 (94), and HMGB1driven MDSC accumulation facilitates metastasis (95). Preliminary studies indicate that HMGB1 mediates its effects on MDSC through RAGE and/or TLR4 (Parker and Ostrand-Rosenberg, unpublished). HMGB1 also binds to other receptors, but it is unknown if MDSC are activated through additional receptors.

\section{8: IL-1 $\beta$, IL-6, and indoleamine 2,3 dioxygenase (IDO)}

The causative relationship between inflammation, cancer, and immune suppression was first proposed following the finding that IL- $1 \beta$ was a potent inducer of MDSC accumulation and suppressive activity (24). Mice bearing 4T1/IL-1 $\beta$, which are 4T1 mammary tumor cells that were transfected to constitutively express high levels of IL-1 $\beta$, exhibit increased MDSC accumulation and more suppressive MDSC compared to mice bearing parental 4T1 tumors. 4T1 tumor-bearing mice that lack the IL-1 receptor antagonist, an inhibitor for IL-1 $\beta$, also develop elevated levels of MDSC that are more suppressive. Similarly, IL-1 $\mathrm{R}^{-/-}$mice display slower tumor growth and their MDSC are 
less suppressive (96-99). Since IL-1 $\beta$ induces the production of other mediators, including VEGF, IL-6, PGE 2 , and GM-CSF, some of the effects of IL-1 $\beta$ on MDSC may be indirect. 4T1 tumor cells transfected to constitutively express IL-6 induce elevated levels of MDSC and restore MDSC levels in tumor-bearing $\mathrm{IL}^{-1 \mathrm{R}^{-/}}$mice, indicating that IL-6 effects on MDSC are either down-stream of IL-1 $\beta$, or have an overlapping mechanism of action with IL-1 $\beta$ (99). Since MDSC produce IL- 6 and IL-1 $\beta$, these studies also raise the question of whether MDSC production of IL- 6 is regulated by IL-1 $\beta$, and if MDSC production of IL- $1 \beta$ enhances MDSC production of IL-6. IDO1, which is utilized by MDSC as an immune suppressive mechanism, also regulates IL-6, and tumor-bearing $\mathrm{IDO1}^{-/-}$mice have less suppressive MDSC, reduced levels of IL-6, and delayed primary tumor growth and metastatic disease (100). Provision of IL-6 to tumor-bearing IDO knockout mice restores MDSC levels and suppressive potency (100).

\section{9: $I L-17$}

IL-17 is a pro-inflammatory cytokine secreted by CD4 Th17 and CD8 Th17 cells. Tumor growth is suppressed and MDSC levels are decreased in IL-17 deficient mice, while administration of IL-17 raises MDSC levels (101, 102). Patients with gastrointestinal cancers show a strong positive correlation between serum IL-17 and MDSC levels, further supporting a role for IL-17 as an inducer of MDSC (103). The effects of IL-17 may be either direct or indirect. Most cells have IL-17 receptors so MDSC may be directly impacted. However, IL-17 triggers the production of IL-6 which in turn activates STAT3, so many effects on MDSC may be directly mediated by IL-6 and indirectly by IL-17 $(101,104)$. 


\subsection{0: Significance of the preceding information to this dissertation}

Since solid tumors are frequently inflamed, understanding how inflammation drives MDSC accumulation and suppressive potency allows the reader to appreciate how inflammation promotes tumor progression through MDSC-mediated suppression of anti-tumor immunity. Additionally, chapter 2 of this dissertation details how MDSC regulate inflammation in solid tumors by cross-talk with macrophages and tumor cells (background on MDSC cross-talk is detailed in section 5). Therefore, the information provided in this section allows the reader to appreciate how my research contributes to what is known about how inflammation is regulated in solid tumors. 


\section{4: MDSC utilize a network of effector and signaling molecules to decrease immune surveillance.}

MDSC utilize multiple suppressive mechanisms to induce a tolerogenic, tumorpromoting environment. MDSC directly suppress T cells by starving them of amino acids, inducing apoptosis, reducing homing to lymph nodes, or by inhibiting their intracellular signaling pathways required for activation. MDSC also indirectly suppress T cells by altering the ability of APC to activate T cells, and by inducing immunosuppressive Tregs. In addition, MDSC impact other cells involved in an antitumor response because they alter the inflammatory milieu in the tumor microenvironment (TME) by cross-talk with macrophages, tumor cells, and mast cells. This section is devoted to explaining suppressive mechanisms that MDSC exert on T cells, and direct signaling events that induce immunosuppressive cell populations or inhibit anti-tumor immunity. Cross-talk between MDSC, macrophages, tumor cells, and mast cells is discussed in section 5. Mechanisms of MDSC-mediated suppression of antitumor immunity are illustrated in Figure 3. 


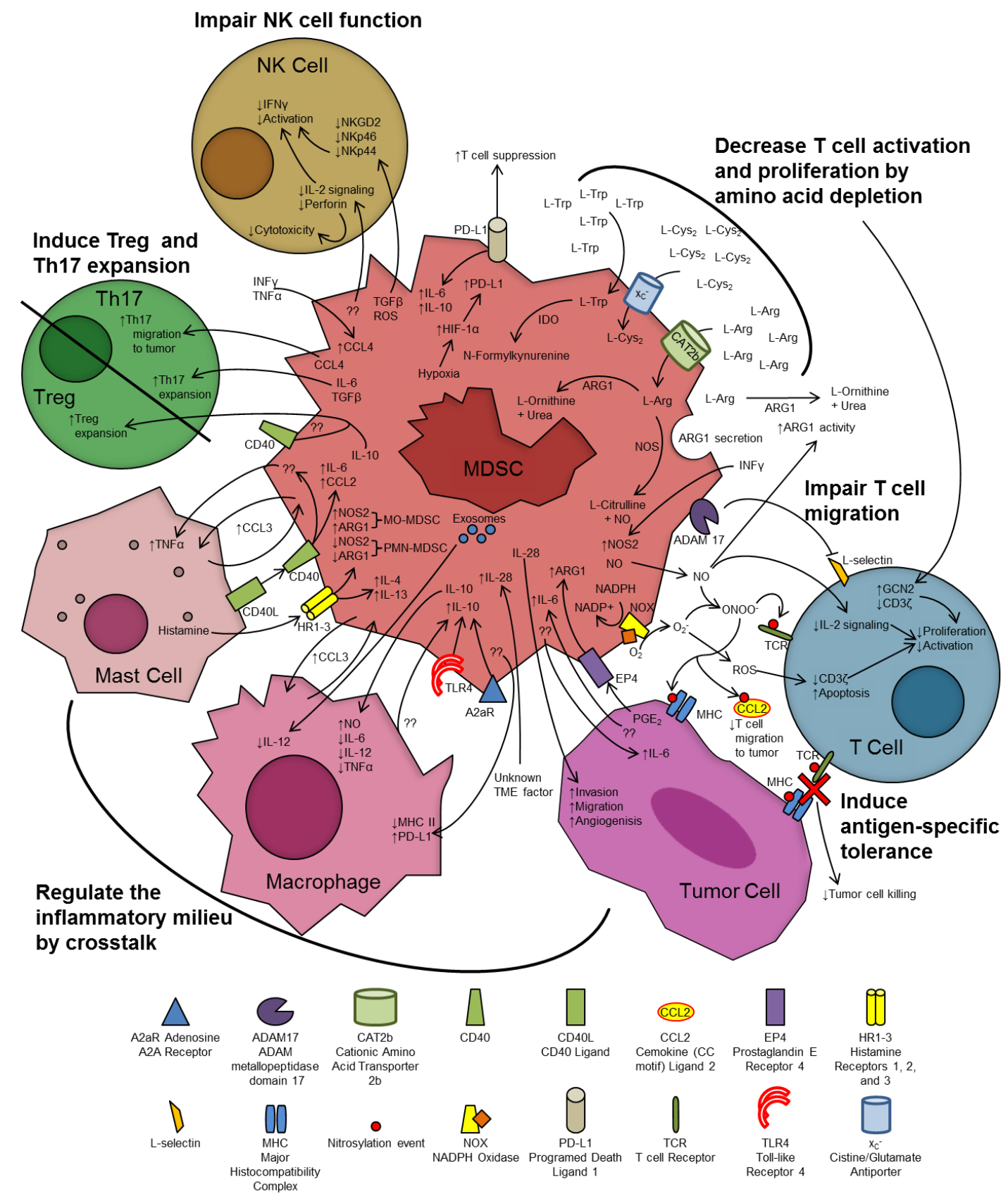

Figure 3: MDSC suppress T cells and regulate the inflammatory milieu by multiple mechanisms. MDSC regulate anti-tumor immunity by: (i) secretion of IL-10, which induces Tregs and drives macrophage polarization to an M2 tumor-promoting phenotype (ii) secretion of IL- 6 and TGF $\beta$, which induces Th17 cells; (iii) production of ROS and TGF $\beta$, which inhibit NK and T cell functions; (iv) degradation of amino acids essential for $\mathrm{T}$ cell activation and proliferation; ( $\mathrm{v}$ ) production of $\mathrm{NO}$ and superoxide $\left(\mathrm{O}_{2}-\right.$ ), which induces apoptosis and inhibits the activation and proliferation of $\mathrm{T}$ cells, and generates PNT that nitrates/nitrosylates MHC and TCR; and (vi) participation in cross-talk with macrophages, tumor cells, and mast cells to generate a pro-tumor environment. Question marks denote an unknown mechanism or signaling molecule. Figure adapted from (55). 


\section{1: MDSC depletion of amino acids}

As discussed earlier in section 2.4, depletion of essential amino acids can inhibit $\mathrm{T}$ cell activation and clonal expansion. MDSC suppress $\mathrm{T}$ cell functions by depleting the local environment of L-arginine (L-Arg), L-tryptophan (L-Trp), and L-cysteine (L-Cys) though different mechanisms.

One of the first suppressive mechanisms attributed to MDSC was the inhibition of T cell activation and proliferation by the depletion of L-Arg. L-Arg is a non-essential amino acid, and is a substrate for several enzymes: (i) nitric oxide synthase (NOS) 1, 2, and 3 which metabolize L-Arg into L-citrulline and nitric oxide (NO); (ii) arginase (ARG) 1 and 2 which convert L-Arg to L-ornithine and urea; (iii) arginine:glycine amidinotransferase which transfers the amidino group from L-Arg to L-glycine, yielding L-ornithine and glycocyamine; and (iv) arginine decarboxylase, which catalyzes the reaction of $\mathrm{L}-\mathrm{Arg}$ to agmatine and $\mathrm{CO}_{2}(105)$.

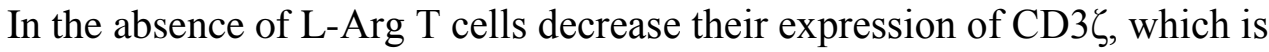
required for signal transduction through the antigen-specific TCR $(106,107)$. L-Argdepleted $T$ cells are arrested in $\mathrm{G}_{0}-\mathrm{G}_{1}$ due to the failure to upregulate cyclin D3 and cyclin-dependent kinase 4 (CDK4). Cyclin D3 and CDK4 are not upregulated due to decreased mRNA stability and lower translation rates (108). Despite their inability to proliferate, L-Arg starved T cells express early activation markers and secrete IL-2, indicating that the early events of T cell activation are not L-Arg dependent (109). In vivo studies confirmed the critical role of MDSC in L-Arg depletion since renal cell carcinoma patients and mice with chronic inflammation have elevated levels of MDSC and low levels of serum L-Arg, which is correlated with decreased T cell activation (110, 
111). Depletion of L-Arg is mediated by ARG1, and MDSC synthesis of ARG1 is regulated by $\mathrm{PGE}_{2}$ (85). Tumor-derived MDSC deplete their local environment of L-Arg by internalizing L-Arg through the cationic amino acid transporter 2B (CAT2B) (112), and by secreting ARG1 (113).

L-Tryptophan metabolism by MDSC also facilitates T cell suppression. MDSC express IDO, which causes T cell suppression by enhancing GCN2 kinase in a similar manner as L-Arg starvation (114). Expression of IDO in MDSC is regulated by STAT3 (115). However, not all MDSC express IDO (100), indicating that IDO is not a universal mechanism utilized by MDSC to suppress T cell activation.

MDSC also prevent T cell activation by sequestering L-Cys. In the extracellular oxidizing environment, L-Cys exists as the dipeptide cystine (L-Cys2). Naïve T cells must acquire L-Cys from APC because they lack the cystine transporter $\mathrm{x}_{\mathrm{c}}{ }^{-}$and therefore cannot import L-Cys2, and cannot de novo synthesize L-Cys because they lack cystathionase, the enzyme that converts methionine to L-Cys. MDSC also lack cystathionase and therefore must scavenge L-Cys2. Since MDSC do not export L-Cys due to their lack of the neutral amino acid transporter ASC, high levels of MDSC quickly deplete their local environment of L-Cys 2 thereby limiting the ability of APC to provide T cells with L-Cys. The role of MDSC and their biological relevance in L-Cys depletion is supported by the correlation between high levels of MDSC and reduced serum L-Cys 2 in tumor-bearing mice (116). Since activated $\mathrm{T}$ cells express $\mathrm{x}_{\mathrm{C}^{-}}$, theoretically they should be resistant to this suppressive mechanism (117). However, since ARG1 production by MDSC suppresses $\mathrm{T}$ cell activation, it is unclear if $\mathrm{T}$ cell up-regulation of $\mathrm{xC}_{\mathrm{C}}^{-}$is functionally relevant. 


\section{2: MDSC production of nitric oxide (NO)}

NOS also catabolizes L-Arg and contributes to MDSC-mediated immune suppression $(105,118)$. MDSC produce NO by the action of NOS2 and NOS3. PMNMDSC are NOS2 $2^{\text {low }} N O S 3^{\text {hi }}$, while M-MDSC are NOS2 $2^{\text {hi }} N O S 3^{\text {low }}(118)$. NOS2 generates more NO than NOS3, and is induced by pro-inflammatory cytokines, endotoxin, hypoxia, and oxidative stress, while NOS3 is constitutively expressed (119).

$\mathrm{NO}$ is labile and reacts with multiple compounds to produce many toxic and regulatory factors. For example, NO reacts with: (i) cysteine thiol groups on proteins and peptides, which form S-nitrosothiols, thereby altering a protein's tertiary structure; (ii) superoxide anions $\left(\mathrm{O}_{2}^{-}\right)$, which form peroxynitrate $\left(\mathrm{PNT}, \mathrm{ONOO}^{-}\right)$, a molecule that alters protein structure; (iii) divalent cations (e.g. $\mathrm{Fe}^{2+}$ and $\mathrm{Zn}^{2+}$ ), which regulate the function of various transcription factors and enzymes; (iv) nucleic acids, which cause mutagenesis; and (v) unsaturated lipids, which lead to the formation of nitrolipids that can have pro- or anti-inflammatory activity (120). Since NO influences many biological processes, it is not surprising that NO is capable of pro- and anti-tumor activity. NO can induce tumorcell apoptosis and inhibit metastasis, or enhance tumor-cell invasion, proliferation, and angiogenesis (119). However, MDSC-produced NO negatively impacts T cells. NO inhibits JAK3, STAT5, ERK, and AKT, which prevents IL-2 signaling, thereby impairing the generation of effector and memory T cells (121). NO directly inhibits these signaling proteins by S-nitrosothiolation, or indirectly by activating guanylate cyclase and cyclicGMP-dependent kinases (122). S-nitrosothiolation of ARG1 enhances ARG1 affinity for L-Arg which subsequently increases ARG1 activity, thereby establishing a synergistic relationship between ARG1- and NO- mediated immune suppression (123). 


\section{3: MDSC production of reactive oxygen species (ROS)}

The NAD $(\mathrm{P}) \mathrm{H}$ oxidase enzyme complex (NOX) is membrane-bound enzyme complex that is utilized by MDSC to suppress T cell activation. This complex catalyzes the production of superoxide through the reduction of oxygen, with $\mathrm{NAD}(\mathrm{P}) \mathrm{H}$ serving as the one electron donor. MDSC from tumor-bearing mice have enhanced expression of the NOX subunits gp91, p22, and p47, and produce more ROS than MDSC from tumor-free mice, and NOX expression is partially regulated by STAT3 (124). Superoxide spontaneously reacts with many molecules to produce a variety of ROS including hydrogen peroxide $\left(\mathrm{H}_{2} \mathrm{O}_{2}\right)$, hydroxyl radical $(\mathrm{OH}-)$, and hypochlorous acid $\left(\mathrm{HOCL}^{-}\right)$. These ROS damage proteins, lipids, and nucleic acids thereby enhancing inflammation and promoting apoptosis. For example, $\mathrm{H}_{2} \mathrm{O}_{2}$ production in cancer patients reduces $\mathrm{T}$ cell production of cytokines and expression of $\mathrm{CD} 3 \zeta$ (125). Superoxide also reacts with NO to form peroxynitrite (PNT), which is produced by PMN-MDSC through the action of gp91 and NOS3 (118). PNT nitrates/nitrosylates the TCR and MHC (126), thereby disrupting TCR-MHC I/peptide binding and rendering tumor cells resistant to CTLmediated apoptosis (127). Due to the short half-life of PNT these reactions are limited to short distances and require close cell-to-cell contact. PNT also reacts with the chemoattractant CCL2, thereby inhibiting T cell infiltration into tumors (128).

4.4: MDSC inhibit T cell migration by down regulating L-and E- selectins.

Activation of tumor-reactive $\mathrm{T}$ cells requires entry of naïve $\mathrm{T}$ cells into tumordraining lymph nodes or migration to the tumor microenvironment. L-selectin mediates the first step in extravasation by facilitating $\mathrm{T}$ cell adhesion to high endothelial venules 
(HEVs). Naïve T cells with low expression of L-selectin do not adhere efficiently to HEVs and fail to enter lymph nodes (Mihich, Evans, Abrams, and Ostrand-Rosenberg, unpublished data). In tumor-bearing mice, MDSC prevent T cell entry into lymph nodes by down-regulating L-selection through their extracellular expression of ADAM17, the enzyme that cleaves L-selectin on naïve T cells (94).

In squamous cell carcinoma patients MDSC also prevent the homing of $\mathrm{T}$ cells to tumor sites by down-regulating E-selectin on tumor vessels. In order for T cells to adhere to tumor vessels and subsequently enter the tumor mass, they must first bind to Eselectin. However, NO produced by MDSC decreases E-selectin levels thereby limiting T cell access to tumor (129).

\section{5: MDSC can express Programmed Death-Ligand 1 (PD-L1)}

As previously discussed in section 2.2, tumor cells escape anti-tumor immunity through their expression of PD-L1. When PD-L1 binds to its receptor PD-1 on T cells, it induces T cell exhaustion/apoptosis. MDSC from some tumor-bearing mice and patients express PD-L1 (130, 131). Some tumor-infiltrating MDSC have elevated expression of PD-L1 due to hypoxia-induced up-regulation of HIF-1 $\alpha$ (132). However, MDSC do not universally express PD-L1, and PD-L1 blockade does not always decrease MDSC suppressive activity (131).

\section{6: MDSC induce Tregs and Th17 cells}

As discussed in section 2.5, Tregs play an important role in the control of immune reactivity against self- and non-self-antigens, and in some animal models they protect 
tumors from anti-tumor immunity. MDSC induce/expand Tregs in vitro and in vivo in multiple tumor models (133-136). MDSC induce Tregs by secreting IL-10 and TGF $\beta$ $(134,137)$, and activate Tregs by presenting tumor-specific antigens in an ARGdependent and TGF $\beta$-independent manner (138). MDSC expression of CD40 is required for MDSC-mediated Treg induction, since CD40-deficient MDSC do not drive Treg expansion (139). Given the link between MDSC and Tregs, therapies targeting MDSC may also reduce Treg populations.

Th17 cells are a pro-inflammatory $\mathrm{CD} 4^{+} \mathrm{T}$ cell subset $\left(\mathrm{CD} 4^{+} \mathrm{ROR} \gamma \mathrm{t}^{+} \mathrm{IL}-17^{+}\right)$. Since they have both pro- and anti-tumor effects, their role in anti-tumor immunity is controversial (140). MDSC induce Th17 cells by producing IL-6 and TGF $\beta$ (104). IFN $\gamma$ or TNF $\alpha$-activated MDSC also recruit Th17 cells through their production of CCL4, which is a Th17 chemoattractant (141). As previously mentioned, IL-17 drives the accumulation of MDSC. Therefore, MDSC and Th17 cells may induce each other.

\section{7: MDSC impair NK cell-mediated cytotoxicity}

MDSC impair NK function via contact-dependent mechanisms. MDSC produce TGF $\beta$ and $\mathrm{H}_{2} \mathrm{O}_{2}$ which decrease NK cell expression of the activating receptors NKG2D, NKp46, and NKp44, thereby making NK cells more difficult to activate $(142,143)$.

MDSC also decrease the ability of NK cells to induce apoptosis in target cells by downregulating NK cell production of perforin which is essential for NK-mediated target cell lysis. In addition, MDSC suppress NK cells by limiting their response to IL-2, a growth factor that enhances NK cell proliferation and cytolytic activity (144). 


\section{8: Significance of the preceding information to this dissertation}

The goal of this dissertation is broaden the breadth of knowledge associated with MDSCmediated suppression of anti-tumor immunity. Therefore, understanding the various mechanisms utilized by MDSC to suppress anti-tumor immunity is necessary for the reader to appreciate how my research contributes to what is known about MDSC-mediated immune suppression. 


\section{5: Cross-talk between MDSC, macrophages, tumor cells, and mast cells enhances inflammation and inhibits anti-tumor immunity}

Both tumor and host (macrophages, dendritic cells, mast cells, MDSC, and fibroblasts) cells within solid tumors participate in cross-talk that regulates the release of pro- and anti-inflammatory cytokines and drive the accumulation and suppressive function of immune-suppressive cells such as Tregs, tumor associated macrophages (TAMs), and MDSC. Additionally, the large number of MDSC induced in tumor-bearing individuals provides ample opportunity for cross-talk to occur in other tissues. This section highlights cellular cross-talk mechanisms that alter the inflammatory milieu and decrease anti-tumor immunity.

\section{1: MDSC and macrophage cross-talk}

As discussed in section 2.6, macrophages can be skewed to a tumoricidal (M1like) phenotype by IFN $\gamma$ and bacterial products such as LPS, or a tumor-promoting (M2like) phenotype by IL-4, IL-13, TGF $\beta$ and IL-10 (48). M1 macrophages express high levels of IL-12, which is an important cytokine for anti-tumor immunity because it stimulates the production of IFN $\gamma$ from $\mathrm{T}$ and NK cells, promotes the development of naïve $\mathrm{CD} 4^{+} \mathrm{Th} 0$ cells to an anti-tumor $\mathrm{Th} 1^{+}$phenotype, and stimulates the growth and cytotoxicity of T cells and NK cells (145). When IFN $\gamma$ and LPS-activate peritoneal macrophages are cultured in vitro in the presence MDSC, MDSC produce high levels of IL-10 (50). 
IL-10 is an anti-inflammatory cytokine with pleiotropic effects on the immune system. It is known to inhibit NF- $\mathrm{kB}$ activity, the synthesis of pro-inflammatory cytokines such as IFN $\gamma$, IL-1, IL-6, and TNF $\alpha$, and APC expression of MHC II and CD86 which decreases T cell activation (146-150). MDSC stimulated with LPS and IFN $\gamma$ produce a basal level of IL-10, however, IL-10 production by MDSC is synergistically enhanced by direct cell-to-cell contact with macrophages (50). MDSC subvert macrophages towards an M2 phenotype through their production of IL-10 which downregulates macrophage production of IL-12 and TNF $\alpha$, while simultaneously enhancing macrophage production of $\mathrm{NO}$, a characteristic of M1 macrophages $(50,51)$. IL-12 down-regulation is mediated by both intact MDSC and MDSC-derived exosomes, which are small (20-100nm) cell-derived vesicles that contain RNA, proteins, lipids, and metabolites (151). MDSC production of IL-10 involves TLR4 signaling, as TLR4 $4^{--}$ MDSC produce low levels of IL-10 (152). MDSC induced in heightened inflammatory conditions (e.g. by 4T1/IL-1 $\beta$ ) have increased levels of IL-10 production (152). Additionally, MDSC also up-regulate PD-L1 on macrophages in the liver (153).

\section{2: MDSC and tumor cell cross-talk}

MDSC and tumor cells also participate in cross-talk. Tumor cells increase MDSC production of IL-6, and in turn, MDSC enhance tumor cell production of IL-6. IL-6 also increases MDSC suppressive activity, but inhibits MDSC production of IL-10 (51). IL-6 promotes tumor progression by enhancing tumor cell development, growth, metastasis, and inhibition of apoptosis, and enhances tumor vascularization (154-156). Therefore there exists a positive feedback loop wherein tumor cells and MDSC induce IL-6 from 
each other, which promotes tumor growth and induction of MDSC accumulation and suppressive activity. Additionally, tumor cells enhance MDSC production of IL-28, which facilitates tumor cell invasion, migration, and angiogenesis (157).

\section{3: MDSC and mast cell cross-talk}

Mast cells and MDSC also interact. MDSC and mast cell cross-talk drives inflammation by increasing production of TNF $\alpha$, CCL3, IL-4, IL-13, IL-6 and CCL2 (158-160). TNF $\alpha$ is a potent pro-inflammatory mediator which contributes to tumor progression in addition to enhancing MDSC accumulation and activity $(161,162)$. CCL3 enhances the migration of leukocytes and fibroblasts to the tumor which promote angiogenesis and metastasis (163), while CCL2 has been implicated in facilitating MDSC migration to tumor and promotes MDSC suppressive activity $(164,165)$ IL-6 and CCL2 production are regulated by ligation of mast cell CD40L to CD40 on MDSC. Activated mast cells release histamine which signals through histamine receptors 1,2 , and 3 on MDSC, and enhances MDSC expression of IL-4 and IL-13. IL-4 and IL-13 signal through STAT6 in MDSC which results in the up-regulation of ARG1 and TGF $\beta$, and enhances macrophage polarization to an M2 phenotype (166-169). Additionally, histamine up-regulates ARG1 and NOS2 in M-MDSC, and decreases ARG1 and NOS2 in PMN-MDSC (160). Since histamine increases M-MDSC production of NO and downregulates immune suppressive mediators of PMN-MDSC, the net effect of histamine is to increase M-MDSC suppressive activity (159). 


\section{4: Significance of the preceding information to this dissertation}

Chapter 2 is focused on how cross-talk between MDSC, macrophages, and tumor cells alters inflammation in the TME. Therefore, the purpose of this section was to provide the reader with a broader understanding of MDSC cross-talk in order to appreciate how my research contributes to what is known about how MDSC function in the TME. 


\section{6: Nuclear factor erythroid 2 (NFE2)-related factor 2 (Nrf2)}

MDSC are present in solid tumors, which is an oxidative environment. Additionally, MDSC utilize ROS to elicit suppression of T cells. However, ROS are nondiscriminatory and could be negatively impacting MDSC. Therefore, we hypothesized that MDSC are protecting themselves from oxidative stress by expressing Nrf2, a transcription factor that regulates the expression of a battery of antioxidant genes. The role of Nrf2 in MDSC survival, accumulation, and suppressive activity is explored in chapter 3. This section of the introduction will detail the structure of Nrf2 and its repressor, Keap1, the activation of Nrf2, Nrf2 binding to the antioxidant response element, and Nrf2 target genes and is illustrated in Figure 4. 
A

Keap1

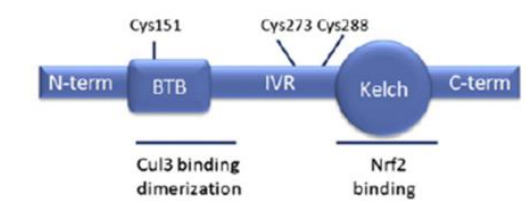

C

C

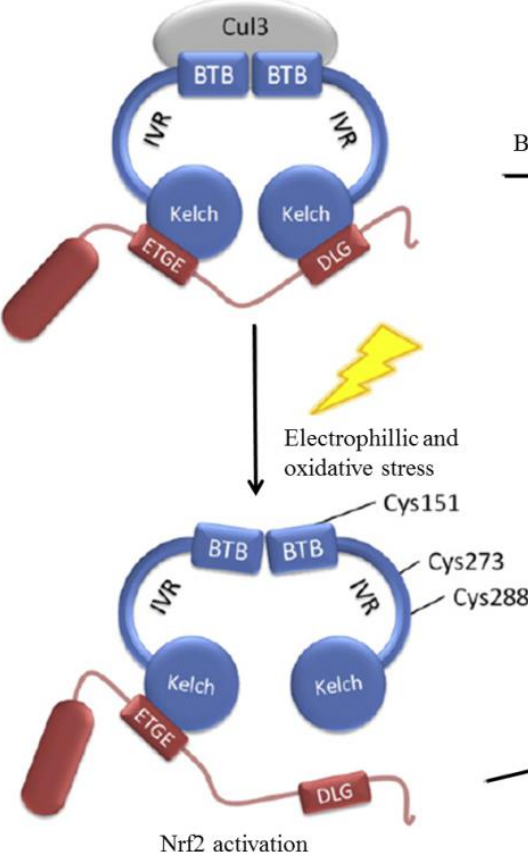

B

Nrf2
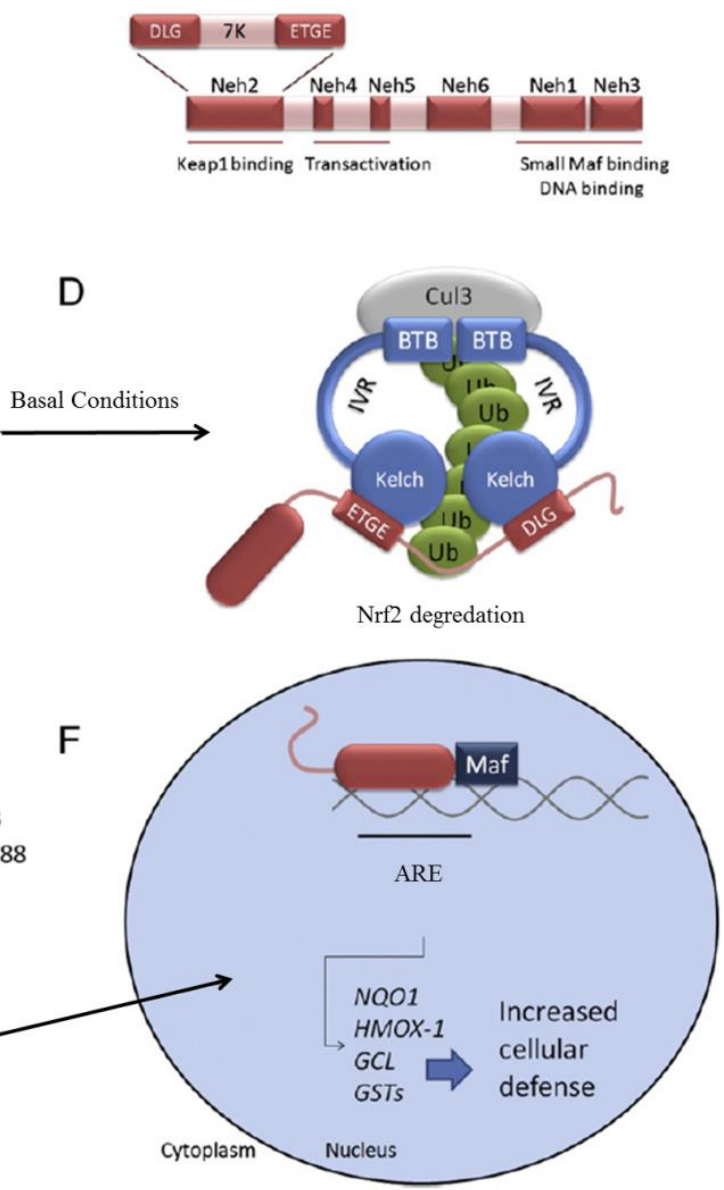

Figure 4: Mechanism for activation of the Keap1-Nrf2 pathway. (A) Keap1 has the following domains: N-terminal domain, BTB domain, IVR, Kelch domain, and Cterminal domain. (B) Nrf2 consists of six Neh domains. The Neh2 domain has two motifs, ETGE and DLG, that bind the Kelch domain on Keap1. (C) Keap1 forms a homodimer that results in a Nrf2-Keap1 complex with a 1:2 ratio. (D) Under basal conditions, Keap1 is an adapter protein for a Cul3-based E3 ubiquitin ligase complex that polyubiquitinates Nrf2. (E) Electrophilic and oxidative stress reacts with key cysteine residues on Keap1 which causes a conformational change that breaks the bond between the DLG domain on Nrf2 and the Kelch domain on Keap1. This change disturbs ubiquitination of Nrf2. (F) Newly synthesized Nrf2 is transported to the nucleus, heterodimerizes with Maf, and promotes transcription of genes regulated by an antioxidant response element. Figure adapted from (170). 


\section{1: The Keap1-Nrf2 pathway}

The primary function of Nrf2 is to mediate the transcription of enzymes that facilitate drug metabolism and deposition, and enzymes that protect against oxidative damage. Nrf2 activity is modulated by a "dedepression" regulatory mechanism where Nrf2 is suppressed under basal conditions by Keap1 (Kelch-like erythroid cell-derived protein with $\mathrm{CNC}$ homology-associated protein 1)-dependent ubiquitination and proteasomal degradation. Nrf2 is activated by oxidants and electrophiles that modify critical thiol residues on Keap1, or by upstream signaling pathways. Once activated, Nrf2 translocates to the nucleus, and binds to the antioxidant response element (ARE) of its target genes, and drives their transcription.

\section{2: Structure of Nrf2}

Nrf2 belongs to the cap 'n' collar (CNC) subfamily of basic leucine zipper (bZip) transcription factors. Nrf2 consists of 589 amino acids and has six evolutionarily conserved domains Neh (Nrf2-ECH homology) domains (Neh 1-6) (Figure 4B). Neh1 contains a bZip motif which consists of a basic region and leucine zipper (L-Zip) structure. The basic region is responsible for DNA binding, and the L-Zip mediates the heterodimerization with a small Maf (171). Neh3 (172), Neh4 (171), and Neh5 (173) are transactivation domains. Neh2 facilitates binding of Nrf2 to Keap1 (174). The Neh2 domain contains two different motifs that bind Keap1, ETGE and DLG, which results in a 1:2 ratio of Nrf2 to Keap1 molecules in the Nrf2:Keap1 complex (Figure 4C) (175). 


\section{3: Structure of Keap1}

Keap1 is a 626 amino acid protein that facilitates the suppression of Nrf2. Keap1 has 5 domains, the N-terminal domain, the Broad complex Tramtrack and Bric- à-Brac (BTB) domain, the intervening region (IVR), the Kelch domain which is also known as double glycine repeats (DGR), and the C-terminal domain (Figure 4A). The BTB domain mediates homodimerization and binding of Keap1 to cullin 3 (Cul3), which serves as a scaffold protein for E3 ubiquitin ligase. The Kelch domain binds actin which anchors Keap1 in the cytoplasm, and is also responsible for binding the Neh2 domain of Nrf2 (176-178). The intervening region is located between the BTB and Kelch domains, and is rich in cysteine residues which serve as a sensor for electrophilic and oxidative stress. Of the 27 cysteine residues that function as stress sensors, three have been deemed critical for mediating the inhibition of Nrf2: Cys151 which is located in the BTB domain, and Cys273 and Cys288 which are located in the IVR (170). Mutations in Cys273 or Cys288 inhibit the ability of Keap1 to repress Nrf2 in vivo under unstressed conditions which results in the accumulation of Nrf2 protein and increased expression of Nrf2 target genes (179). Cys151 plays a different role compared to Cys273 and Cys288, and is essential for detecting certain electrophiles. Sulforaphane, tert-butylhydroquinone, N-iodoacetyl-Nbiotinylhexylenediamine, diethylmaleate, and ebselen activate Nrf2 in a Keap1 Cys151dependent manner (170).

\section{4: Mechanisms of Nrf2 activation}

It is evident that modification of cysteine residues leads to the accumulation of $\mathrm{Nrf} 2$ in the nucleus and regulates expression of Nrf2 target genes. Under basal 
conditions, Keap1 functions as an adapter protein in the Cul3-based ubiquitin ligase complex, which rapidly ubiquitinates Nrf2 and subsequently targets it for proteasomal degradation (Figure 4C) $(180,181)$. During electrophilic or oxidative stress, modification of specific Keap1 cysteines leads to a conformational change in Keap1, resulting in the detachment of the DLG motif on Nrf2 from the Kelch domain on Keap1 (Figure 4E). The binding between ETGE motif and the second Kelch domain remains, and the result is the ubiquitination of $\operatorname{Nrf} 2$ is disturbed $(175,182,183)$. This process deactivates the Keap1 complex, and permits newly synthesized Nrf2 to translocate to the nucleus and modulate the transcription of ARE-regulated genes. Additionally, Nrf2 can also be stabilized through direct phosphorylation by kinases involved in inflammatory signaling cascades (e.g. KRAS, MYC, PKC, ERK, MAPK, and p38) (184-189).

\section{5: Nrf2 heterodimerizes with other transcription factors and mediates the transcription}

\section{of ARE-regulated genes}

The ARE is a 41-base-pair enhancer element in the promoter of genes that are regulated Nrf2. It contains a 16-base-pair consensus sequence that resembles the DNAbinding elements of several other bZip proteins (190-192). The ARE consensus sequence shares homology with the TPA (12-0-tetradecanoylphorbol-13-aceta) response element which is recognized by AP-1 proteins, the NFE2-binding motif, and the Maf protein recognition element (MARE) which is recognized by Maf dimers. bZip proteins including AP-1, NFE2, Nrf1, Nrf2, Nrf3, Bach1 and 2, small Mafs, and CREB/ATF exhibit overlapping binding activities of these elements, and cross-interactions of these bZip proteins through heterodimerization increases their gene target repertoire. Therefore 
the genes regulated by Nrf2 are mediated by the presence of Nrf2 binding partners. Known Nrf2 binding partners include Jun (c-Jun, Jun-D, and Jun-B) and small Maf (MafG, MafK, and MafF) (193-198).

\section{6: Genes regulated by Nrf2}

Target genes of Nrf2 have been identified by using gene expression profiling in $\mathrm{Nrf}^{-/-}$mice. These genes can be classified into several categories including xenobiotic metabolizing enzymes such as $\mathrm{NAD}(\mathrm{P}) \mathrm{H}$ :quinone oxidoreductase (NQO1) and glutathione S-transferases (GST), xenobiotic transport enzymes such as multidrug resistance-associated protein, glutathione synthesis genes such as glutamate-cysteine ligase and $\mathrm{xCT}$, and ROS catabolism genes such as superoxide dismutase, among others (199).

\section{7: Significance of the preceding information to this dissertation}

MDSC are present in the oxidative TME and utilize ROS to suppress T cell activation and proliferation. Since ROS are nondiscriminatory in their ability to damage multiple cell types, we hypothesized that MDSC utilize Nrf2 to protect themselves from oxidative stress. Therefore, the information provided in this section was provided as essential background information for the reader so they appreciate the role $\mathrm{Nrf} 2$ is playing in MDSC. 


\section{Goal of dissertation}

My research has been focused on understanding how tumor-induced immune suppression is modulated by MDSC. The TME is a complex and frequently inflamed microenvironment. The inflammation is driven by pro-inflammatory mediators which are secreted by tumor cells, various tumor-infiltrating lymphocytes, tumor-associated fibroblasts, and myeloid cells such as macrophages, dendritic cells, and MDSC (67). Some of these cells engage in cross-talk with each other resulting in the release of proinflammatory cytokines (e.g. IL-1, IL-6, IL-17, TNF $)$ ), chemokines (e.g. CCL2, CXCL5, CXCL12), growth factors (e.g. TGF $\beta$, GM-CSF, VEGF), and other effector molecules (e.g. S100A8/A9, High Mobility Group Box 1)(70, 200, 201). It is established that crosstalk between MDSC and macrophages impacts anti-tumor immunity (50); however, interactions between tumor cells and MDSC or macrophages are less well studied. Therefore, chapter 2 examines the impact of tumor cells, MDSC, and macrophages on each other and how their effects upon each other promote inflammation and tumor progression.

In addition to being highly inflamed, the TME is oxidatively stressed due to hypoxia, the presence of reactive oxygen species (ROS), and multiple pro-inflammatory mediators (202). MDSC are present in the TME, and also produce high of levels of ROS to facilitate T cell suppression. Although ROS are toxic to most cells, MDSC are able to survive and function despite their exposure to oxidative stress. Therefore we hypothesized that MDSC are protecting themselves from oxidative stress by expressing Nrf2, a transcription factor that responds to oxidative stress and regulates the expression 
of antioxidant genes (199). Chapter 3 focuses on how Nrf2 impacts MDSC suppressive activity, survival, and accumulation, and identifies Nrf2 as another molecule that aids in promoting MDSC suppressive activity and thereby enhances tumor progression. 


\section{References}

1. Society, A. C. 2015. Cancer Facts \& Figures. Atlanta: American Cancer Society.

2. Weigelt, B., J. L. Peterse, and L. J. van 't Veer. 2005. Breast cancer metastasis: markers and models. Nature reviews. Cancer 5: 591-602.

3. Burgdorf, S., A. Kautz, V. Bohnert, P. A. Knolle, and C. Kurts. 2007. Distinct pathways of antigen uptake and intracellular routing in CD4 and CD8 T cell activation. Science 316: 612-616.

4. Priyadharshini, B., D. L. Greiner, and M. A. Brehm. 2012. T-cell activation and transplantation tolerance. Transplant Reviews 26: 212-222.

5. Liao, W., J. X. Lin, and W. J. Leonard. 2011. IL-2 family cytokines: new insights into the complex roles of IL-2 as a broad regulator of T helper cell differentiation. Curr Opin Immunol 23: 598-604.

6. Schroder, K., P. J. Hertzog, T. Ravasi, and D. A. Hume. 2004. Interferon-gamma: an overview of signals, mechanisms and functions. J Leukoc Biol 75: 163-189.

7. Basse, P. H., T. L. Whiteside, and R. B. Herberman. 2002. Cancer immunotherapy with interleukin-2-activated natural killer cells. Mol Biotechnol 21: 161-170.

8. Ostrand-Rosenberg, S., P. Sinha, D. W. Beury, and V. K. Clements. 2012. Crosstalk between myeloid-derived suppressor cells (MDSC), macrophages, and dendritic cells enhances tumor-induced immune suppression. Semin Cancer Biol 22: $275-281$. 
9. Noelle, R. J., and E. C. Snow. 1991. T helper cell-dependent B cell activation. FASEB Journal 5: 2770-2776.

10. P, E. 1909. Über den jetzigen Stand der Karzinomforschung. Beiträge zur experimentellen Pathologie und Chemotherapie: 117-164.

11. Burnet, M. 1957. Cancer; a biological approach. I. The processes of control. British medical journal 1: 779-786.

12. Engel, A. M., I. M. Svane, S. Mouritsen, J. Rygaard, J. Clausen, and O. Werdelin. 1996. Methylcholanthrene-induced sarcomas in nude mice have short induction times and relatively low levels of surface MHC class I expression. Apmis 104: 629-639.

13. Shankaran, V., H. Ikeda, A. T. Bruce, J. M. White, P. E. Swanson, L. J. Old, and R. D. Schreiber. 2001. IFNgamma and lymphocytes prevent primary tumour development and shape tumour immunogenicity. Nature 410: 1107-1111.

14. Smyth, M. J., K. Y. Thia, S. E. Street, E. Cretney, J. A. Trapani, M. Taniguchi, T. Kawano, S. B. Pelikan, N. Y. Crowe, and D. I. Godfrey. 2000. Differential tumor surveillance by natural killer (NK) and NKT cells. The Journal of experimental medicine 191: 661-668.

15. Street, S. E., E. Cretney, and M. J. Smyth. 2001. Perforin and interferon-gamma activities independently control tumor initiation, growth, and metastasis. Blood 97: 192-197.

16. Street, S. E., J. A. Trapani, D. MacGregor, and M. J. Smyth. 2002. Suppression of lymphoma and epithelial malignancies effected by interferon gamma. The Journal of experimental medicine 196: 129-134. 
17. van den Broek, M. E., D. Kagi, F. Ossendorp, R. Toes, S. Vamvakas, W. K. Lutz, C. J. Melief, R. M. Zinkernagel, and H. Hengartner. 1996. Decreased tumor surveillance in perforin-deficient mice. The Journal of experimental medicine 184: $1781-1790$.

18. Ostrand-Rosenberg, S. 2008. Immune surveillance: a balance between protumor and antitumor immunity. Current opinion in genetics \& development 18: 11-18.

19. Dunn, G. P., L. J. Old, and R. D. Schreiber. 2004. The three Es of cancer immunoediting. Аnпu Rev Immunol 22: 329-360.

20. Pierotti MA, S. G., Croce CM., ed. 2003. Mechanisms of oncogene activation. Hamilton (ON): BC Decker.

21. Swann, J. B., and M. J. Smyth. 2007. Immune surveillance of tumors. The Journal of clinical investigation 117: 1137-1146.

22. Schreiber, R. D., L. J. Old, and M. J. Smyth. 2011. Cancer immunoediting: integrating immunity's roles in cancer suppression and promotion. Science 331: $1565-1570$.

23. Rabinovich, G. A., D. Gabrilovich, and E. M. Sotomayor. 2007. Immunosuppressive strategies that are mediated by tumor cells. Annu Rev Immunol 25: 267-296.

24. Ostrand-Rosenberg, S., and P. Sinha. 2009. Myeloid-derived suppressor cells: linking inflammation and cancer. J Immunol 182: 4499-4506.

25. Marincola, F. M., P. Shamamian, T. B. Simonis, A. Abati, J. Hackett, T. O'Dea, P. Fetsch, J. Yannelli, N. P. Restifo, J. J. Mule, and e. al. 1994. Locus-specific 
analysis of human leukocyte antigen class I expression in melanoma cell lines. $J$ Immunother Emphasis Tumor Immunol 16: 13-23.

26. Rivoltini, L., M. Carrabba, V. Huber, C. Castelli, L. Novellino, P. Dalerba, R. Mortarini, G. Arancia, A. Anichini, S. Fais, and G. Parmiani. 2002. Immunity to cancer: attack and escape in T lymphocyte-tumor cell interaction. Immunol Rev 188: 97-113.

27. Smith-Garvin, J. E., G. A. Koretzky, and M. S. Jordan. 2009. T cell activation. Annu Rev Immunol 27: 591-619.

28. Chen, L. 2004. Co-inhibitory molecules of the B7-CD28 family in the control of T-cell immunity. Nat Rev Immunol 4: 336-347.

29. Alegre, M. L., K. A. Frauwirth, and C. B. Thompson. 2001. T-cell regulation by CD28 and CTLA-4. Nat Rev Immunol 1: 220-228.

30. Walker, L. S., and D. M. Sansom. 2011. The emerging role of CTLA4 as a cellextrinsic regulator of T cell responses. Nat Rev Immunol 11: 852-863.

31. Qureshi, O. S., Y. Zheng, K. Nakamura, K. Attridge, C. Manzotti, E. M. Schmidt, J. Baker, L. E. Jeffery, S. Kaur, Z. Briggs, T. Z. Hou, C. E. Futter, G. Anderson, L. S. Walker, and D. M. Sansom. 2011. Trans-endocytosis of CD80 and CD86: a molecular basis for the cell-extrinsic function of CTLA-4. Science 332: 600-603.

32. Dong, H., S. E. Strome, D. R. Salomao, H. Tamura, F. Hirano, D. B. Flies, P. C. Roche, J. Lu, G. Zhu, K. Tamada, V. A. Lennon, E. Celis, and L. Chen. 2002. Tumor-associated B7-H1 promotes T-cell apoptosis: a potential mechanism of immune evasion. Nat Med 8: 793-800. 
33. Lee, S. J., B. C. Jang, S. W. Lee, Y. I. Yang, S. I. Suh, Y. M. Park, S. Oh, J. G. Shin, S. Yao, L. Chen, and I. H. Choi. 2006. Interferon regulatory factor-1 is prerequisite to the constitutive expression and IFN-gamma-induced upregulation of B7-H1 (CD274). FEBS Lett 580: 755-762.

34. Driessens, G., J. Kline, and T. F. Gajewski. 2009. Costimulatory and coinhibitory receptors in anti-tumor immunity. Immunol Rev 229: 126-144.

35. Rozali, E. N., S. V. Hato, B. W. Robinson, R. A. Lake, and W. J. Lesterhuis. 2012. Programmed death ligand 2 in cancer-induced immune suppression. Clin Dev Immunol 2012: 656340.

36. Massague, J. 2008. TGFbeta in Cancer. Cell 134: 215-230.

37. Thomas, D. A., and J. Massagué. 2005. TGF-beta directly targets cytotoxic T cell functions during tumor evasion of immune surveillance. Cancer Cell 8: 369-380.

38. Wang, R., and D. R. Green. 2012. Metabolic checkpoints in activated T cells. Nat Immunol 13: 907-915.

39. Mellor, A. L., and D. H. Munn. 2004. IDO expression by dendritic cells: tolerance and tryptophan catabolism. Nat Rev Immunol 4: 762-774.

40. Prendergast, G. C. 2008. Immune escape as a fundamental trait of cancer: focus on IDO. Oncogene 27: 3889-3900.

41. Sakaguchi, S., N. Sakaguchi, J. Shimizu, S. Yamazaki, T. Sakihama, M. Itoh, Y. Kuniyasu, T. Nomura, M. Toda, and T. Takahashi. 2001. Immunologic tolerance maintained by CD25+CD4+ regulatory T cells: their common role in controlling autoimmunity, tumor immunity, and transplantation tolerance. Immunol Rev 182: 18-32. 
42. Vignali, D. A., L. W. Collison, and C. J. Workman. 2008. How regulatory T cells work. Nat Rev Immunol 8: 523-532.

43. Yamaguchi, T., and S. Sakaguchi. 2006. Regulatory T cells in immune surveillance and treatment of cancer. Semin Cancer Biol 16: 115-123.

44. Zhao, D. M., A. M. Thornton, R. J. DiPaolo, and E. M. Shevach. 2006. Activated CD4+CD25+ T cells selectively kill B lymphocytes. Blood 107: 3925-3932.

45. Cao, X., S. F. Cai, T. A. Fehniger, J. Song, L. I. Collins, D. R. Piwnica-Worms, and T. J. Ley. 2007. Granzyme B and perforin are important for regulatory T cellmediated suppression of tumor clearance. Immunity 27: 635-646.

46. Ren, X., F. Ye, Z. Jiang, Y. Chu, S. Xiong, and Y. Wang. 2007. Involvement of cellular death in TRAIL/DR5-dependent suppression induced by CD4(+)CD25(+) regulatory T cells. Cell Death Differ 14: 2076-2084.

47. Read, S., V. Malmstrom, and F. Powrie. 2000. Cytotoxic T lymphocyteassociated antigen 4 plays an essential role in the function of CD25(+)CD4(+) regulatory cells that control intestinal inflammation. The Journal of experimental medicine 192: 295-302.

48. Sica, A., and A. Mantovani. 2012. Macrophage plasticity and polarization: in vivo veritas. J Clin Invest 122: 787-795.

49. Noy, R., and J. W. Pollard. 2014. Tumor-associated macrophages: from mechanisms to therapy. Immunity 41: 49-61.

50. Sinha, P., V. K. Clements, S. K. Bunt, S. M. Albelda, and S. Ostrand-Rosenberg. 2007. Cross-talk between myeloid-derived suppressor cells and macrophages subverts tumor immunity toward a type 2 response. J Immunol 179: 977-983. 
51. Beury, D. W., K. H. Parker, M. Nyandjo, P. Sinha, K. A. Carter, and S. OstrandRosenberg. 2014. Cross-talk among myeloid-derived suppressor cells, macrophages, and tumor cells impacts the inflammatory milieu of solid tumors. $J$ Leukoc Biol 96: 1109-1118.

52. Gabrilovich, D. I., M. P. Velders, E. M. Sotomayor, and W. M. Kast. 2001. Mechanism of immune dysfunction in cancer mediated by immature Gr-1+ myeloid cells. J Immunol 166: 5398-5406.

53. Bronte, V., E. Apolloni, A. Cabrelle, R. Ronca, P. Serafini, P. Zamboni, N. P. Restifo, and P. Zanovello. 2000. Identification of a CD11b(+)/Gr-1(+)/CD31(+) myeloid progenitor capable of activating or suppressing CD8(+) T cells. Blood 96: $3838-3846$.

54. Almand, B., J. I. Clark, E. Nikitina, J. van Beynen, N. R. English, S. C. Knight, D. P. Carbone, and D. I. Gabrilovich. 2001. Increased production of immature myeloid cells in cancer patients: a mechanism of immunosuppression in cancer. $J$ Immunol 166: 678-689.

55. Parker, K. H., D. W. Beury, and S. Ostrand-Rosenberg. 2015. Myeloid-Derived Suppressor Cells: Critical Cells Driving Immune Suppression in the Tumor Microenvironment. Adv Cancer Res 128: 95-139.

56. Balkwill, F., and A. Mantovani. 2001. Inflammation and cancer: back to Virchow? Lancet 357: 539-545.

57. DeNardo, D. G., P. Andreu, and L. M. Coussens. 2010. Interactions between lymphocytes and myeloid cells regulate pro- versus anti-tumor immunity. Cancer Metastasis Rev 29: 309-316. 
58. Grivennikov, S. I., F. R. Greten, and M. Karin. 2010. Immunity, inflammation, and cancer. Cell 140: 883-899.

59. Qian, B. Z., and J. W. Pollard. 2010. Macrophage diversity enhances tumor progression and metastasis. Cell 141: 39-51.

60. Punturieri, A., E. Szabo, T. L. Croxton, S. D. Shapiro, and S. M. Dubinett. 2009. Lung cancer and chronic obstructive pulmonary disease: needs and opportunities for integrated research. J Natl Cancer Inst 101: 554-559.

61. Zong, W. X., and C. B. Thompson. 2006. Necrotic death as a cell fate. Genes Dev 20: $1-15$.

62. Zitvogel, L., L. Apetoh, F. Ghiringhelli, and G. Kroemer. 2008. Immunological aspects of cancer chemotherapy. Nat Rev Immunol 8: 59-73.

63. von Roon, A. C., G. Reese, J. Teare, V. Constantinides, A. W. Darzi, and P. P. Tekkis. 2007. The risk of cancer in patients with Crohn's disease. Dis Colon Rectum 50: 839-855.

64. Karin, M. 2006. Nuclear factor-kappaB in cancer development and progression. Nature 441: 431-436.

65. Wu, S., K. J. Rhee, E. Albesiano, S. Rabizadeh, X. Wu, H. R. Yen, D. L. Huso, F. L. Brancati, E. Wick, F. McAllister, F. Housseau, D. M. Pardoll, and C. L. Sears. 2009. A human colonic commensal promotes colon tumorigenesis via activation of T helper type $17 \mathrm{~T}$ cell responses. Nat Med 15: 1016-1022.

66. Mostafa, M. H., S. A. Sheweita, and P. J. O'Connor. 1999. Relationship between schistosomiasis and bladder cancer. Clin Microbiol Rev 12: 97-111. 
67. Shiao, S. L., A. P. Ganesan, H. S. Rugo, and L. M. Coussens. 2011. Immune microenvironments in solid tumors: new targets for therapy. Genes Dev 25: 25592572.

68. Cao, C., Y. Gu, C. Zhu, T. Palmai-Pallag, F. Lan, Z. Chen, W. Li, H. Shen, and S. Ying. 2014. Potential roles of eosinophils in cancer therapy: epidemiological studies, experimental models, and clinical pathology. Recent Pat Anticancer Drug Discov 9: 241-248.

69. Khazaie, K., N. R. Blatner, M. W. Khan, F. Gounari, E. Gounaris, K. Dennis, A. Bonertz, F. N. Tsai, M. J. Strouch, E. Cheon, J. D. Phillips, P. Beckhove, and D. J. Bentrem. 2011. The significant role of mast cells in cancer. Cancer Metastasis Rev 30: 45-60.

70. Gabrilovich, D. I., S. Ostrand-Rosenberg, and V. Bronte. 2012. Coordinated regulation of myeloid cells by tumours. Nat Rev Immunol 12: 253-268.

71. Gabrilovich, D., T. Ishida, T. Oyama, S. Ran, V. Kravtsov, S. Nadaf, and D. P. Carbone. 1998. Vascular endothelial growth factor inhibits the development of dendritic cells and dramatically affects the differentiation of multiple hematopoietic lineages in vivo. Blood 92: 4150-4166.

72. Kusmartsev, S., E. Eruslanov, H. Kübler, T. Tseng, Y. Sakai, Z. Su, S. Kaliberov, A. Heiser, C. Rosser, P. Dahm, D. Siemann, and J. Vieweg. 2008. Oxidative stress regulates expression of VEGFR1 in myeloid cells: link to tumor-induced immune suppression in renal cell carcinoma. J Immunol 181: 346-353. 
73. Kujawski, M., M. Kortylewski, H. Lee, A. Herrmann, H. Kay, and H. Yu. 2008. Stat3 mediates myeloid cell-dependent tumor angiogenesis in mice. J Clin Invest 118: 3367-3377.

74. Bergers, G., R. Brekken, G. McMahon, T. H. Vu, T. Itoh, K. Tamaki, K. Tanzawa, P. Thorpe, S. Itohara, Z. Werb, and D. Hanahan. 2000. Matrix metalloproteinase-9 triggers the angiogenic switch during carcinogenesis. Nat Cell Biol 2: 737-744.

75. Melani, C., S. Sangaletti, F. M. Barazzetta, Z. Werb, and M. P. Colombo. 2007. Amino-biphosphonate-mediated MMP-9 inhibition breaks the tumor-bone marrow axis responsible for myeloid-derived suppressor cell expansion and macrophage infiltration in tumor stroma. Cancer Res 67: 11438-11446.

76. Serafini, P., R. Carbley, K. A. Noonan, G. Tan, V. Bronte, and I. Borrello. 2004. High-dose granulocyte-macrophage colony-stimulating factor-producing vaccines impair the immune response through the recruitment of myeloid suppressor cells. Cancer Res 64: 6337-6343.

77. Morales, J. K., M. Kmieciak, K. L. Knutson, H. D. Bear, and M. H. Manjili. 2010. GM-CSF is one of the main breast tumor-derived soluble factors involved in the differentiation of CD11b-Gr1- bone marrow progenitor cells into myeloid-derived suppressor cells. Breast Cancer Res Treat 123: 39-49.

78. Nefedova, Y., M. Huang, S. Kusmartsev, R. Bhattacharya, P. Cheng, R. Salup, R. Jove, and D. Gabrilovich. 2004. Hyperactivation of STAT3 is involved in abnormal differentiation of dendritic cells in cancer. J Immunol 172: 464-474. 
79. Lieschke, G. J., D. Grail, G. Hodgson, D. Metcalf, E. Stanley, C. Cheers, K. J. Fowler, S. Basu, Y. F. Zhan, and A. R. Dunn. 1994. Mice lacking granulocyte colony-stimulating factor have chronic neutropenia, granulocyte and macrophage progenitor cell deficiency, and impaired neutrophil mobilization. Blood 84: 17371746.

80. Okazaki, T., S. Ebihara, M. Asada, A. Kanda, H. Sasaki, and M. Yamaya. 2006. Granulocyte colony-stimulating factor promotes tumor angiogenesis via increasing circulating endothelial progenitor cells and Gr1+CD11b+ cells in cancer animal models. Int Immunol 18: 1-9.

81. Kowanetz, M., X. Wu, J. Lee, M. Tan, T. Hagenbeek, X. Qu, L. Yu, J. Ross, N. Korsisaari, T. Cao, H. Bou-Reslan, D. Kallop, R. Weimer, M. J. Ludlam, J. S. Kaminker, Z. Modrusan, N. van Bruggen, F. V. Peale, R. Carano, Y. G. Meng, and N. Ferrara. 2010. Granulocyte-colony stimulating factor promotes lung metastasis through mobilization of Ly6G+Ly6C+ granulocytes. Proc Natl Acad Sci U S A 107: 21248-21255.

82. Dolcetti, L., E. Peranzoni, S. Ugel, I. Marigo, A. Fernandez Gomez, C. Mesa, M. Geilich, G. Winkels, E. Traggiai, A. Casati, F. Grassi, and V. Bronte. 2010. Hierarchy of immunosuppressive strength among myeloid-derived suppressor cell subsets is determined by GM-CSF. Eur J Immunol 40: 22-35.

83. Sinha, P., V. K. Clements, A. M. Fulton, and S. Ostrand-Rosenberg. 2007. Prostaglandin E2 promotes tumor progression by inducing myeloid-derived suppressor cells. Cancer Res 67: 4507-4513. 
84. Eruslanov, E., I. Daurkin, J. Ortiz, J. Vieweg, and S. Kusmartsev. 2010. Pivotal Advance: Tumor-mediated induction of myeloid-derived suppressor cells and M2-polarized macrophages by altering intracellular $\mathrm{PGE}_{2}$ catabolism in myeloid cells. J Leukoc Biol 88: 839-848.

85. Rodriguez, P. C., C. P. Hernandez, D. Quiceno, S. M. Dubinett, J. Zabaleta, J. B. Ochoa, J. Gilbert, and A. C. Ochoa. 2005. Arginase I in myeloid suppressor cells is induced by COX-2 in lung carcinoma. $J$ Exp Med 202: 931-939.

86. Obermajer, N., R. Muthuswamy, J. Lesnock, R. P. Edwards, and P. Kalinski. 2011. Positive feedback between PGE2 and COX2 redirects the differentiation of human dendritic cells toward stable myeloid-derived suppressor cells. Blood 118: 5498-5505.

87. Marigo, I., E. Bosio, S. Solito, C. Mesa, A. Fernandez, L. Dolcetti, S. Ugel, N. Sonda, S. Bicciato, E. Falisi, F. Calabrese, G. Basso, P. Zanovello, E. Cozzi, S. Mandruzzato, and V. Bronte. 2010. Tumor-induced tolerance and immune suppression depend on the C/EBPbeta transcription factor. Immunity 32: 790-802.

88. Thevenot, P. T., R. A. Sierra, P. L. Raber, A. A. Al-Khami, J. Trillo-Tinoco, P. Zarreii, A. C. Ochoa, Y. Cui, L. Del Valle, and P. C. Rodriguez. 2014. The stressresponse sensor chop regulates the function and accumulation of myeloid-derived suppressor cells in tumors. Immunity 41: 389-401.

89. Markiewski, M. M., R. A. DeAngelis, F. Benencia, S. K. Ricklin-Lichtsteiner, A. Koutoulaki, C. Gerard, G. Coukos, and J. D. Lambris. 2008. Modulation of the antitumor immune response by complement. Nat Immunol 9: 1225-1235. 
90. Cheng, P., C. A. Corzo, N. Luetteke, B. Yu, S. Nagaraj, M. M. Bui, M. Ortiz, W. Nacken, C. Sorg, T. Vogl, J. Roth, and D. I. Gabrilovich. 2008. Inhibition of dendritic cell differentiation and accumulation of myeloid-derived suppressor cells in cancer is regulated by S100A9 protein. J Exp Med 205: 2235-2249.

91. Sinha, P., C. Okoro, D. Foell, H. H. Freeze, S. Ostrand-Rosenberg, and G. Srikrishna. 2008. Proinflammatory S100 proteins regulate the accumulation of myeloid-derived suppressor cells. J Immunol 181: 4666-4675.

92. Bresnick, A. R., D. J. Weber, and D. B. Zimmer. 2015. S100 proteins in cancer. Nat Rev Cancer 15: 96-109.

93. Bianchi, M. E., and A. A. Manfredi. 2007. High-mobility group box 1 (HMGB1) protein at the crossroads between innate and adaptive immunity. Immunol Rev 220: $35-46$.

94. Parker, K., P. Sinha, L. Horn, V. Clements, and S. Ostrand-Rosenberg. 2014. HMGB1 enhances immune suppression by facilitating the differentiation and suppressive activity of myeloid-derived suppressor cells. Cancer Res.

95. Li, W., K. Wu, E. Zhao, L. Shi, R. Li, P. Zhang, Y. Yin, X. Shuai, G. Wang, and K. Tao. 2013. HMGB1 recruits myeloid derived suppressor cells to promote peritoneal dissemination of colon cancer after resection. Biochem Biophys Res Commun 436: 156-161.

96. Elkabets, M., V. S. Ribeiro, C. A. Dinarello, S. Ostrand-Rosenberg, J. P. Di Santo, R. N. Apte, and C. A. Vosshenrich. 2010. IL-1 $\beta$ regulates a novel myeloidderived suppressor cell subset that impairs NK cell development and function. Eur J Immunol 40: 3347-3357. 
97. Bunt, S. K., P. Sinha, V. K. Clements, J. Leips, and S. Ostrand-Rosenberg. 2006. Inflammation induces myeloid-derived suppressor cells that facilitate tumor progression. J Immunol 176: 284-290.

98. Song, X., Y. Krelin, T. Dvorkin, O. Bjorkdahl, S. Segal, C. A. Dinarello, E. Voronov, and R. N. Apte. 2005. CD11b+/Gr-1+ immature myeloid cells mediate suppression of T cells in mice bearing tumors of IL-1beta-secreting cells. $J$ Immunol 175: 8200-8208.

99. Bunt, S. K., L. Yang, P. Sinha, V. K. Clements, J. Leips, and S. OstrandRosenberg. 2007. Reduced inflammation in the tumor microenvironment delays the accumulation of myeloid-derived suppressor cells and limits tumor progression. Cancer Res 67: 10019-10026.

100. Smith, C., M. Y. Chang, K. H. Parker, D. W. Beury, J. B. DuHadaway, H. E. Flick, J. Boulden, E. Sutanto-Ward, A. P. Soler, L. D. Laury-Kleintop, L. Mandik-Nayak, R. Metz, S. Ostrand-Rosenberg, G. C. Prendergast, and A. J. Muller. 2012. IDO is a nodal pathogenic driver of lung cancer and metastasis development. Cancer Discov 2: 722-735.

101. Wang, L., T. Yi, M. Kortylewski, D. M. Pardoll, D. Zeng, and H. Yu. 2009. IL-17 can promote tumor growth through an IL-6-Stat3 signaling pathway. J Exp Med 206: $1457-1464$.

102. He, D., H. Li, N. Yusuf, C. A. Elmets, J. Li, J. D. Mountz, and H. Xu. 2010. IL-17 promotes tumor development through the induction of tumor promoting microenvironments at tumor sites and myeloid-derived suppressor cells. $J$ Immunol 184: 2281-2288. 
103. Yazawa, T., M. Shibata, K. Gonda, T. Machida, S. Suzuki, A. Kenjo, I. Nakamura, T. Tsuchiya, Y. Koyama, K. Sakurai, T. Shimura, R. Tomita, H. Ohto, M. Gotoh, and S. Takenoshita. 2013. Increased IL-17 production correlates with immunosuppression involving myeloid-derived suppressor cells and nutritional impairment in patients with various gastrointestinal cancers. Mol Clin Oncol 1: 675-679.

104. Chatterjee, S., S. Das, P. Chakraborty, A. Manna, M. Chatterjee, and S. K. Choudhuri. 2013. Myeloid derived suppressor cells (MDSCs) can induce the generation of Th17 response from naive CD4+ T cells. Immunobiology 218: 718724.

105. Bronte, V., and P. Zanovello. 2005. Regulation of immune responses by Larginine metabolism. Nat Rev Immunol 5: 641-654.

106. Zea, A. H., P. C. Rodriguez, K. S. Culotta, C. P. Hernandez, J. DeSalvo, J. B. Ochoa, H. J. Park, J. Zabaleta, and A. C. Ochoa. 2004. L-Arginine modulates CD3zeta expression and $\mathrm{T}$ cell function in activated human $\mathrm{T}$ lymphocytes. Cell Immunol 232: 21-31.

107. Rodriguez, P. C., A. H. Zea, K. S. Culotta, J. Zabaleta, J. B. Ochoa, and A. C. Ochoa. 2002. Regulation of T cell receptor CD3zeta chain expression by Larginine. J Biol Chem 277: 21123-21129.

108. Rodriguez, P. C., C. P. Hernandez, K. Morrow, R. Sierra, J. Zabaleta, D. D. Wyczechowska, and A. C. Ochoa. 2010. L-arginine deprivation regulates cyclin D3 mRNA stability in human T cells by controlling HuR expression. J Immunol 185: 5198-5204. 
109. Fletcher, M., M. E. Ramirez, R. A. Sierra, P. Raber, P. Thevenot, A. A. AlKhami, D. Sanchez-Pino, C. Hernandez, D. D. Wyczechowska, A. C. Ochoa, and P. C. Rodriguez. 2015. 1-Arginine Depletion Blunts Antitumor T-cell Responses by Inducing Myeloid-Derived Suppressor Cells. Cancer Res 75: 275-283.

110. Ezernitchi, A. V., I. Vaknin, L. Cohen-Daniel, O. Levy, E. Manaster, A. Halabi, E. Pikarsky, L. Shapira, and M. Baniyash. 2006. TCR zeta down-regulation under chronic inflammation is mediated by myeloid suppressor cells differentially distributed between various lymphatic organs. J Immunol 177: 4763-4772.

111. Zea, A. H., P. C. Rodriguez, M. B. Atkins, C. Hernandez, S. Signoretti, J. Zabaleta, D. McDermott, D. Quiceno, A. Youmans, A. O'Neill, J. Mier, and A. C. Ochoa. 2005. Arginase-producing myeloid suppressor cells in renal cell carcinoma patients: a mechanism of tumor evasion. Cancer Res 65: 3044-3048.

112. Rodriguez, P. C., D. G. Quiceno, J. Zabaleta, B. Ortiz, A. H. Zea, M. B. Piazuelo, A. Delgado, P. Correa, J. Brayer, E. M. Sotomayor, S. Antonia, J. B. Ochoa, and A. C. Ochoa. 2004. Arginase I production in the tumor microenvironment by mature myeloid cells inhibits T-cell receptor expression and antigen-specific Tcell responses. Cancer Res 64: 5839-5849.

113. Rodriguez, P. C., M. S. Ernstoff, C. Hernandez, M. Atkins, J. Zabaleta, R. Sierra, and A. C. Ochoa. 2009. Arginase I-producing myeloid-derived suppressor cells in renal cell carcinoma are a subpopulation of activated granulocytes. Cancer Res 69: $1553-1560$. 
114. Munn, D. H., M. D. Sharma, B. Baban, H. P. Harding, Y. Zhang, D. Ron, and A. L. Mellor. 2005. GCN2 kinase in T cells mediates proliferative arrest and anergy induction in response to indoleamine 2,3-dioxygenase. Immunity 22: 633-642.

115. Yu, J., W. Du, F. Yan, Y. Wang, H. Li, S. Cao, W. Yu, C. Shen, J. Liu, and X. Ren. 2013. Myeloid-derived suppressor cells suppress antitumor immune responses through IDO expression and correlate with lymph node metastasis in patients with breast cancer. J Immunol 190: 3783-3797.

116. Srivastava, M. K., P. Sinha, V. K. Clements, P. Rodriguez, and S. OstrandRosenberg. 2010. Myeloid-derived suppressor cells inhibit T-cell activation by depleting cystine and cysteine. Cancer Res 70: 68-77.

117. Levring, T. B., A. K. Hansen, B. L. Nielsen, M. Kongsbak, M. R. von Essen, A. Woetmann, N. Odum, C. M. Bonefeld, and C. Geisler. 2012. Activated human CD4+ T cells express transporters for both cysteine and cystine. Sci Rep 2: 266.

118. Raber, P. L., P. Thevenot, R. Sierra, D. Wyczechowska, D. Halle, M. E. Ramirez, A. C. Ochoa, M. Fletcher, C. Velasco, A. Wilk, K. Reiss, and P. C. Rodriguez. 2014. Subpopulations of myeloid-derived suppressor cells impair T cell responses through independent nitric oxide-related pathways. Int J Cancer 134: 2853-2864.

119. Fukumura, D., S. Kashiwagi, and R. K. Jain. 2006. The role of nitric oxide in tumour progression. Nat Rev Cancer 6: 521-534.

120. Bogdan, C. 2015. Nitric oxide synthase in innate and adaptive immunity: an update. Trends Immunol. 
121. Mazzoni, A., V. Bronte, A. Visintin, J. H. Spitzer, E. Apolloni, P. Serafini, P. Zanovello, and D. M. Segal. 2002. Myeloid suppressor lines inhibit T cell responses by an NO-dependent mechanism. J Immunol 168: 689-695.

122. Serafini, P. 2013. Myeloid derived suppressor cells in physiological and pathological conditions: the good, the bad, and the ugly. Immunol Res 57: 172184.

123. Santhanam, L., H. K. Lim, V. Miriel, T. Brown, M. Patel, S. Balanson, S. Ryoo, M. Anderson, K. Irani, F. Khanday, L. Di Costanzo, D. Nyhan, J. M. Hare, D. W. Christianson, R. Rivers, A. Shoukas, and D. E. Berkowitz. 2007. Inducible NO synthase dependent S-nitrosylation and activation of arginase1 contribute to agerelated endothelial dysfunction. Circ Res 101: 692-702.

124. Corzo, C. A., M. J. Cotter, P. Cheng, F. Cheng, S. Kusmartsev, E. Sotomayor, T. Padhya, T. V. McCaffrey, J. C. McCaffrey, and D. I. Gabrilovich. 2009. Mechanism regulating reactive oxygen species in tumor-induced myeloid-derived suppressor cells. J Immunol 182: 5693-5701.

125. Schmielau, J., and O. J. Finn. 2001. Activated granulocytes and granulocytederived hydrogen peroxide are the underlying mechanism of suppression of t-cell function in advanced cancer patients. Cancer Res 61: 4756-4760.

126. Lu, T., and D. I. Gabrilovich. 2012. Molecular pathways: tumor-infiltrating myeloid cells and reactive oxygen species in regulation of tumor microenvironment. Clin Cancer Res 18: 4877-4882.

127. Lu, T., R. Ramakrishnan, S. Altiok, J. I. Youn, P. Cheng, E. Celis, V. Pisarev, S. Sherman, M. B. Sporn, and D. Gabrilovich. 2011. Tumor-infiltrating myeloid 
cells induce tumor cell resistance to cytotoxic T cells in mice. J Clin Invest 121: 4015-4029.

128. Molon, B., S. Ugel, F. Del Pozzo, C. Soldani, S. Zilio, D. Avella, A. De Palma, P. Mauri, A. Monegal, M. Rescigno, B. Savino, P. Colombo, N. Jonjic, S. Pecanic, L. Lazzarato, R. Fruttero, A. Gasco, V. Bronte, and A. Viola. 2011. Chemokine nitration prevents intratumoral infiltration of antigen-specific T cells. J Exp Med 208: 1949-1962.

129. Gehad, A. E., M. K. Lichtman, C. D. Schmults, J. E. Teague, A. W. Calarese, Y. Jiang, R. Watanabe, and R. A. Clark. 2012. Nitric oxide-producing myeloidderived suppressor cells inhibit vascular E-selectin expression in human squamous cell carcinomas. J Invest Dermatol 132: 2642-2651.

130. Zhang, B., Z. Wang, L. Wu, M. Zhang, W. Li, J. Ding, J. Zhu, H. Wei, and K. Zhao. 2013. Circulating and tumor-infiltrating myeloid-derived suppressor cells in patients with colorectal carcinoma. PLoS One 8: e57114.

131. Youn, J. I., S. Nagaraj, M. Collazo, and D. I. Gabrilovich. 2008. Subsets of myeloid-derived suppressor cells in tumor-bearing mice. J Immunol 181: 57915802.

132. Noman, M. Z., G. Desantis, B. Janji, M. Hasmim, S. Karray, P. Dessen, V. Bronte, and S. Chouaib. 2014. PD-L1 is a novel direct target of HIF-1alpha, and its blockade under hypoxia enhanced MDSC-mediated T cell activation. $J$ Exp Med 211: 781-790.

133. MacDonald, K. P., V. Rowe, A. D. Clouston, J. K. Welply, R. D. Kuns, J. L. Ferrara, R. Thomas, and G. R. Hill. 2005. Cytokine expanded myeloid precursors 
function as regulatory antigen-presenting cells and promote tolerance through IL10-producing regulatory T cells. J Immunol 174: 1841-1850.

134. Huang, B., P. Y. Pan, Q. Li, A. I. Sato, D. E. Levy, J. Bromberg, C. M. Divino, and S. H. Chen. 2006. Gr-1+CD115+ immature myeloid suppressor cells mediate the development of tumor-induced T regulatory cells and T-cell anergy in tumorbearing host. Cancer Res 66: 1123-1131.

135. Adeegbe, D., P. Serafini, V. Bronte, A. Zoso, C. Ricordi, and L. Inverardi. 2011. In vivo induction of myeloid suppressor cells and CD4(+)Foxp3(+) T regulatory cells prolongs skin allograft survival in mice. Cell Transplant 20: 941-954.

136. Zoso, A., E. M. Mazza, S. Bicciato, S. Mandruzzato, V. Bronte, P. Serafini, and L. Inverardi. 2014. Human fibrocytic myeloid-derived suppressor cells express IDO and promote tolerance via Treg-cell expansion. Eur J Immunol 44: 33073319.

137. Hoechst, B., L. A. Ormandy, M. Ballmaier, F. Lehner, C. Kruger, M. P. Manns, T. F. Greten, and F. Korangy. 2008. A new population of myeloid-derived suppressor cells in hepatocellular carcinoma patients induces CD4(+)CD25(+)Foxp3(+) T cells. Gastroenterology 135: 234-243.

138. Serafini, P., S. Mgebroff, K. Noonan, and I. Borrello. 2008. Myeloid-derived suppressor cells promote cross-tolerance in B-cell lymphoma by expanding regulatory T cells. Cancer Res 68: 5439-5449.

139. Pan, P. Y., G. Ma, K. J. Weber, J. Ozao-Choy, G. Wang, B. Yin, C. M. Divino, and S. H. Chen. 2010. Immune stimulatory receptor CD40 is required for T-cell 
suppression and $\mathrm{T}$ regulatory cell activation mediated by myeloid-derived suppressor cells in cancer. Cancer Res 70: 99-108.

140. Ye, J., R. S. Livergood, and G. Peng. 2013. The role and regulation of human Th17 cells in tumor immunity. Am J Pathol 182: 10-20.

141. Ortiz, M. L., V. Kumar, A. Martner, S. Mony, L. Donthireddy, T. Condamine, J. Seykora, S. C. Knight, G. Malietzis, G. H. Lee, M. Moorghen, B. Lenox, N. Luetteke, E. Celis, and D. Gabrilovich. 2015. Immature myeloid cells directly contribute to skin tumor development by recruiting IL-17-producing CD4+ T cells. J Exp Med.

142. Mao, Y., D. Sarhan, A. Steven, B. Seliger, R. Kiessling, and A. Lundqvist. 2014. Inhibition of tumor-derived prostaglandin-e2 blocks the induction of myeloidderived suppressor cells and recovers natural killer cell activity. Clin Cancer Res 20: 4096-4106.

143. Elkabets, M., V. S. Ribeiro, C. A. Dinarello, S. Ostrand-Rosenberg, J. P. Di Santo, R. N. Apte, and C. A. Vosshenrich. 2010. IL-1beta regulates a novel myeloid-derived suppressor cell subset that impairs NK cell development and function. Eur J Immunol 40: 3347-3357.

144. Liu, C., S. Yu, J. Kappes, J. Wang, W. E. Grizzle, K. R. Zinn, and H. G. Zhang. 2007. Expansion of spleen myeloid suppressor cells represses NK cell cytotoxicity in tumor-bearing host. Blood 109: 4336-4342.

145. Lasek, W., R. Zagozdzon, and M. Jakobisiak. 2014. Interleukin 12: still a promising candidate for tumor immunotherapy? Cancer immunology, immunotherapy : CII 63: 419-435. 
146. Fiorentino, D. F., A. Zlotnik, T. R. Mosmann, M. Howard, and A. O'Garra. 1991. IL-10 inhibits cytokine production by activated macrophages. J Immunol 147: $3815-3822$.

147. Wang, P., P. Wu, M. I. Siegel, R. W. Egan, and M. M. Billah. 1995. Interleukin (IL)-10 inhibits nuclear factor kappa B (NF kappa B) activation in human monocytes. IL-10 and IL-4 suppress cytokine synthesis by different mechanisms. J Biol Chem 270: 9558-9563.

148. Fiorentino, D. F., A. Zlotnik, P. Vieira, T. R. Mosmann, M. Howard, K. W. Moore, and A. O'Garra. 1991. IL-10 acts on the antigen-presenting cell to inhibit cytokine production by Th1 cells. J Immunol 146: 3444-3451.

149. Buelens, C., F. Willems, A. Delvaux, G. Pierard, J. P. Delville, T. Velu, and M. Goldman. 1995. Interleukin-10 differentially regulates B7-1 (CD80) and B7-2 (CD86) expression on human peripheral blood dendritic cells. Eur J Immunol 25: $2668-2672$.

150. Huang, C. J., B. R. Stevens, R. B. Nielsen, P. N. Slovin, X. Fang, D. R. Nelson, and J. W. Skimming. 2002. Interleukin-10 inhibition of nitric oxide biosynthesis involves suppression of CAT-2 transcription. Nitric Oxide 6: 79-84.

151. Burke, M., W. Choksawangkarn, N. Edwards, S. Ostrand-Rosenberg, and C. Fenselau. 2014. Exosomes from myeloid-derived suppressor cells carry biologically active proteins. J Proteome Res 13: 836-843.

152. Bunt, S. K., V. K. Clements, E. M. Hanson, P. Sinha, and S. Ostrand-Rosenberg. 2009. Inflammation enhances myeloid-derived suppressor cell cross-talk by signaling through Toll-like receptor 4. J Leukoc Biol 85: 996-1004. 
153. Ilkovitch, D., and D. M. Lopez. 2009. The liver is a site for tumor-induced myeloid-derived suppressor cell accumulation and immunosuppression. Cancer Res 69: 5514-5521.

154. Becker, C., M. C. Fantini, S. Wirtz, A. Nikolaev, H. A. Lehr, P. R. Galle, S. RoseJohn, and M. F. Neurath. 2005. IL-6 signaling promotes tumor growth in colorectal cancer. Cell Cycle 4: 217-220.

155. Su, Y. W., T. X. Xie, D. Sano, and J. N. Myers. 2011. IL-6 stabilizes Twist and enhances tumor cell motility in head and neck cancer cells through activation of casein kinase 2. PLoS One 6: e19412.

156. Santer, F. R., K. Malinowska, Z. Culig, and I. T. Cavarretta. 2010. Interleukin-6 trans-signalling differentially regulates proliferation, migration, adhesion and maspin expression in human prostate cancer cells. Endocr Relat Cancer 17: 241253.

157. Mucha, J., K. Majchrzak, B. Taciak, E. Hellmen, and M. Krol. 2014. MDSCs mediate angiogenesis and predispose canine mammary tumor cells for metastasis via IL-28/IL-28RA (IFN-lambda) signaling. PLoS One 9: e103249.

158. Saleem, S. J., R. K. Martin, J. K. Morales, J. L. Sturgill, D. R. Gibb, L. Graham, H. D. Bear, M. H. Manjili, J. J. Ryan, and D. H. Conrad. 2012. Cutting edge: mast cells critically augment myeloid-derived suppressor cell activity. J Immunol 189: $511-515$.

159. Danelli, L., B. Frossi, G. Gri, F. Mion, C. Guarnotta, L. Bongiovanni, C. Tripodo, L. Mariuzzi, S. Marzinotto, A. Rigoni, U. Blank, M. P. Colombo, and C. E. Pucillo. 2015. Mast cells boost myeloid-derived suppressor cell activity and 
contribute to the development of tumor-favoring microenvironment. Cancer Immunol Res 3: 85-95.

160. Martin, R. K., S. J. Saleem, L. Folgosa, H. B. Zellner, S. R. Damle, G. K. Nguyen, J. J. Ryan, H. D. Bear, A. M. Irani, and D. H. Conrad. 2014. Mast cell histamine promotes the immunoregulatory activity of myeloid-derived suppressor cells. J Leukoc Biol 96: 151-159.

161. Balkwill, F. 2009. Tumour necrosis factor and cancer. Nat Rev Cancer 9: 361371.

162. Zhao, X., L. Rong, X. Li, X. Liu, J. Deng, H. Wu, X. Xu, U. Erben, P. Wu, U. Syrbe, J. Sieper, and Z. Qin. 2012. TNF signaling drives myeloid-derived suppressor cell accumulation. J Clin Invest 122: 4094-4104.

163. Wu, Y., Y. Y. Li, K. Matsushima, T. Baba, and N. Mukaida. 2008. CCL3-CCR5 axis regulates intratumoral accumulation of leukocytes and fibroblasts and promotes angiogenesis in murine lung metastasis process. Journal of Immunology 181: 6384-6393.

164. Chun, E., S. Lavoie, M. Michaud, C. A. Gallini, J. Kim, G. Soucy, R. Odze, J. N. Glickman, and W. S. Garrett. 2015. CCL2 Promotes Colorectal Carcinogenesis by Enhancing Polymorphonuclear Myeloid-Derived Suppressor Cell Population and Function. Cell Rep 12: 244-257.

165. Lesokhin, A. M., T. M. Hohl, S. Kitano, C. Cortez, D. Hirschhorn-Cymerman, F. Avogadri, G. A. Rizzuto, J. J. Lazarus, E. G. Pamer, A. N. Houghton, T. Merghoub, and J. D. Wolchok. 2012. Monocytic CCR2(+) myeloid-derived 
suppressor cells promote immune escape by limiting activated CD8 T-cell infiltration into the tumor microenvironment. Cancer Res 72: 876-886.

166. Sinha, P., V. K. Clements, and S. Ostrand-Rosenberg. 2005. Reduction of myeloid-derived suppressor cells and induction of M1 macrophages facilitate the rejection of established metastatic disease. J Immunol 174: 636-645.

167. Sinha, P., V. K. Clements, and S. Ostrand-Rosenberg. 2005. Interleukin-13regulated M2 macrophages in combination with myeloid suppressor cells block immune surveillance against metastasis. Cancer Res 65: 11743-11751.

168. Bronte, V., P. Serafini, C. De Santo, I. Marigo, V. Tosello, A. Mazzoni, D. M. Segal, C. Staib, M. Lowel, G. Sutter, M. P. Colombo, and P. Zanovello. 2003. IL4-induced arginase 1 suppresses alloreactive T cells in tumor-bearing mice. $J$ Immunol 170: 270-278.

169. Terabe, M., S. Matsui, J. M. Park, M. Mamura, N. Noben-Trauth, D. D. Donaldson, W. Chen, S. M. Wahl, S. Ledbetter, B. Pratt, J. J. Letterio, W. E. Paul, and J. A. Berzofsky. 2003. Transforming growth factor-beta production and myeloid cells are an effector mechanism through which CD1d-restricted T cells block cytotoxic $\mathrm{T}$ lymphocyte-mediated tumor immunosurveillance: abrogation prevents tumor recurrence. J Exp Med 198: 1741-1752.

170. Kansanen, E., H. K. Jyrkkanen, and A. L. Levonen. 2012. Activation of stress signaling pathways by electrophilic oxidized and nitrated lipids. Free radical biology \& medicine 52: 973-982.

171. Itoh, K., T. Chiba, S. Takahashi, T. Ishii, K. Igarashi, Y. Katoh, T. Oyake, N. Hayashi, K. Satoh, I. Hatayama, M. Yamamoto, and Y. Nabeshima. 1997. An 
Nrf2/small Maf heterodimer mediates the induction of phase II detoxifying enzyme genes through antioxidant response elements. Biochemical and biophysical research communications 236: 313-322.

172. Nioi, P., T. Nguyen, P. J. Sherratt, and C. B. Pickett. 2005. The carboxy-terminal Neh3 domain of Nrf2 is required for transcriptional activation. Molecular and cellular biology 25: 10895-10906.

173. Zhang, J., T. Hosoya, A. Maruyama, K. Nishikawa, J. M. Maher, T. Ohta, H. Motohashi, A. Fukamizu, S. Shibahara, K. Itoh, and M. Yamamoto. 2007. Nrf2 Neh5 domain is differentially utilized in the transactivation of cytoprotective genes. Biochem J 404: 459-466.

174. Itoh, K., N. Wakabayashi, Y. Katoh, T. Ishii, K. Igarashi, J. D. Engel, and M. Yamamoto. 1999. Keap1 represses nuclear activation of antioxidant responsive elements by Nrf2 through binding to the amino-terminal Neh2 domain. Genes \& development 13: 76-86.

175. Tong, K. I., Y. Katoh, H. Kusunoki, K. Itoh, T. Tanaka, and M. Yamamoto. 2006. Keap1 recruits Neh2 through binding to ETGE and DLG motifs: characterization of the two-site molecular recognition model. Molecular and cellular biology 26: 2887-2900.

176. Li, X., D. Zhang, M. Hannink, and L. J. Beamer. 2004. Crystal structure of the Kelch domain of human Keap1. J Biol Chem 279: 54750-54758.

177. Ogura, T., K. I. Tong, K. Mio, Y. Maruyama, H. Kurokawa, C. Sato, and M. Yamamoto. 2010. Keap1 is a forked-stem dimer structure with two large spheres 
enclosing the intervening, double glycine repeat, and C-terminal domains. Proc Natl Acad Sci U S A 107: 2842-2847.

178. Padmanabhan, B., K. I. Tong, T. Ohta, Y. Nakamura, M. Scharlock, M. Ohtsuji, M. I. Kang, A. Kobayashi, S. Yokoyama, and M. Yamamoto. 2006. Structural basis for defects of Keap1 activity provoked by its point mutations in lung cancer. Mol Cell 21: 689-700.

179. Yamamoto, T., T. Suzuki, A. Kobayashi, J. Wakabayashi, J. Maher, H. Motohashi, and M. Yamamoto. 2008. Physiological significance of reactive cysteine residues of Keap1 in determining Nrf2 activity. Molecular and cellular biology 28: 2758-2770.

180. Cullinan, S. B., J. D. Gordan, J. Jin, J. W. Harper, and J. A. Diehl. 2004. The Keap1-BTB protein is an adaptor that bridges Nrf2 to a Cul3-based E3 ligase: oxidative stress sensing by a Cul3-Keap1 ligase. Molecular and cellular biology 24: $8477-8486$.

181. Kobayashi, A., M. I. Kang, H. Okawa, M. Ohtsuji, Y. Zenke, T. Chiba, K. Igarashi, and M. Yamamoto. 2004. Oxidative stress sensor Keap1 functions as an adaptor for Cul3-based E3 ligase to regulate proteasomal degradation of Nrf2. Molecular and cellular biology 24: 7130-7139.

182. Tong, K. I., A. Kobayashi, F. Katsuoka, and M. Yamamoto. 2006. Two-site substrate recognition model for the Keap1-Nrf2 system: a hinge and latch mechanism. Biol Chem 387: 1311-1320.

183. Tong, K. I., B. Padmanabhan, A. Kobayashi, C. Shang, Y. Hirotsu, S. Yokoyama, and M. Yamamoto. 2007. Different electrostatic potentials define ETGE and DLG 
motifs as hinge and latch in oxidative stress response. Molecular and cellular biology 27: 7511-7521.

184. Bloom, D. A., and A. K. Jaiswal. 2003. Phosphorylation of Nrf2 at Ser40 by protein kinase $\mathrm{C}$ in response to antioxidants leads to the release of $\mathrm{Nrf} 2$ from INrf2, but is not required for Nrf2 stabilization/accumulation in the nucleus and transcriptional activation of antioxidant response element-mediated NAD(P)H:quinone oxidoreductase-1 gene expression. J Biol Chem 278: 4467544682.

185. Buckley, B. J., Z. M. Marshall, and A. R. Whorton. 2003. Nitric oxide stimulates Nrf2 nuclear translocation in vascular endothelium. Biochem Biophys Res Commun 307: 973-979.

186. Cullinan, S. B., D. Zhang, M. Hannink, E. Arvisais, R. J. Kaufman, and J. A. Diehl. 2003. Nrf2 is a direct PERK substrate and effector of PERK-dependent cell survival. Mol Cell Biol 23: 7198-7209.

187. Huang, H. C., T. Nguyen, and C. B. Pickett. 2002. Phosphorylation of Nrf2 at Ser-40 by protein kinase $\mathrm{C}$ regulates antioxidant response element-mediated transcription. J Biol Chem 277: 42769-42774.

188. Yu, R., W. Lei, S. Mandlekar, M. J. Weber, C. J. Der, J. Wu, and A. N. Kong. 1999. Role of a mitogen-activated protein kinase pathway in the induction of phase II detoxifying enzymes by chemicals. J Biol Chem 274: 27545-27552.

189. Zipper, L. M., and R. T. Mulcahy. 2000. Inhibition of ERK and p38 MAP kinases inhibits binding of Nrf2 and induction of GCS genes. Biochem Biophys Res Commun 278: 484-492. 
190. Ma, Q., and X. He. 2012. Molecular basis of electrophilic and oxidative defense: promises and perils of Nrf2. Pharmacol Rev 64: 1055-1081.

191. Nioi, P., M. McMahon, K. Itoh, M. Yamamoto, and J. D. Hayes. 2003. Identification of a novel Nrf2-regulated antioxidant response element (ARE) in the mouse $\mathrm{NAD}(\mathrm{P}) \mathrm{H}$ :quinone oxidoreductase 1 gene: reassessment of the $\mathrm{ARE}$ consensus sequence. Biochem J 374: 337-348.

192. Wasserman, W. W., and W. E. Fahl. 1997. Functional antioxidant responsive elements. Proc Natl Acad Sci U S A 94: 5361-5366.

193. Itoh, K., T. Chiba, S. Takahashi, T. Ishii, K. Igarashi, Y. Katoh, T. Oyake, N. Hayashi, K. Satoh, I. Hatayama, M. Yamamoto, and Y. Nabeshima. 1997. An Nrf2/small Maf heterodimer mediates the induction of phase II detoxifying enzyme genes through antioxidant response elements. Biochem Biophys Res Commun 236: 313-322.

194. Kwak, M. K., J. M. Cho, B. Huang, S. Shin, and T. W. Kensler. 2007. Role of increased expression of the proteasome in the protective effects of sulforaphane against hydrogen peroxide-mediated cytotoxicity in murine neuroblastoma cells. Free Radic Biol Med 43: 809-817.

195. Alam, J., D. Stewart, C. Touchard, S. Boinapally, A. M. Choi, and J. L. Cook. 1999. Nrf2, a Cap'n'Collar transcription factor, regulates induction of the heme oxygenase-1 gene. J Biol Chem 274: 26071-26078.

196. Nguyen, T., H. C. Huang, and C. B. Pickett. 2000. Transcriptional regulation of the antioxidant response element. Activation by Nrf2 and repression by MafK. $J$ Biol Chem 275: 15466-15473. 
197. Wild, A. C., H. R. Moinova, and R. T. Mulcahy. 1999. Regulation of gammaglutamylcysteine synthetase subunit gene expression by the transcription factor Nrf2. J Biol Chem 274: 33627-33636.

198. Venugopal, R., and A. K. Jaiswal. 1998. Nrf2 and Nrf1 in association with Jun proteins regulate antioxidant response element-mediated expression and coordinated induction of genes encoding detoxifying enzymes. Oncogene 17: 3145-3156.

199. Ma, Q. 2013. Role of nrf2 in oxidative stress and toxicity. Annu Rev Pharmacol Toxicol 53: 401-426.

200. Yang, L., Y. Pang, and H. L. Moses. 2010. TGF-beta and immune cells: an important regulatory axis in the tumor microenvironment and progression. Trends Immunol 31: 220-227.

201. Viola, A., A. Sarukhan, V. Bronte, and B. Molon. 2012. The pros and cons of chemokines in tumor immunology. Trends Immunol 33: 496-504.

202. Liou, G. Y., and P. Storz. 2010. Reactive oxygen species in cancer. Free Radic Res 44: 479-496. 


\title{
Chapter 2: Cross-talk among myeloid-derived suppressor cells, macrophages, and tumor cells impacts the inflammatory milieu of solid tumors ${ }^{1}$
}

\section{Footnotes}

1. This chapter was published in Journal of Leukocyte Biology. The published version is Appendix 1.

\begin{abstract}
Myeloid-derived suppressor cells (MDSC) and macrophages are present in most solid tumors and are important drivers of immune suppression and inflammation. It is established that cross-talk between MDSC and macrophages impacts anti-tumor immunity; however, interactions between tumor cells and MDSC or macrophages are less well studied. To examine potential interactions between these cells we studied the impact of MDSC, macrophages, and four murine tumor cell lines on each other, both in vitro and in vivo. We focused on IL-6, IL-10, IL-12, TNF $\alpha$, and nitric oxide (NO) because these molecules are produced by macrophages, MDSC, and many tumor cells, are present in most solid tumors, and regulate inflammation. In vitro studies demonstrated that MDSCproduced IL-10 decreased macrophage IL- 6 and TNF $\alpha$, and increased nitric oxide (NO). IL-6 indirectly regulated MDSC IL-10. Tumor cells increased MDSC IL-6 and vice versa. Tumor cells also increased macrophage IL-6 and NO, and decreased macrophage TNF $\alpha$. Tumor-cell-driven macrophage IL-6 was reduced by MDSC, and tumor cells and
\end{abstract}


MDSC enhanced macrophage NO. In vivo analysis of solid tumors identified IL-6 and IL-10 as the dominant cytokines and demonstrated that these molecules were predominantly produced by stromal cells. These results suggest that inflammation within solid tumors is regulated by the ratio of tumor cells to MDSC and macrophages, and that interactions of these cells have the potential to significantly alter the inflammatory milieu within the tumor microenvironment.

\section{Introduction}

Solid tumors are a complex and frequently inflamed environment. The inflammation is driven by pro-inflammatory mediators which are secreted by tumor cells, various tumorinfiltrating lymphocytes, tumor-associated fibroblasts, and myeloid cells such as macrophages, dendritic cells, and myeloid-derived suppressor cells (MDSC) (1). Some of these cells engage in cross-talk with each other resulting in the release of proinflammatory cytokines (e.g. IL-1, IL-6, IL-17, TNF $\alpha$ ), chemokines (e.g. CCL2, CXCL5, CXCL12), growth factors (e.g. TGF $\beta$, GM-CSF, VEGF), and other effector molecules (e.g. S100A8/A9, High Mobility Group Box 1)(2-4). These factors, in turn, induce the accumulation and enhance the function of immune-suppressive cells such as regulatory $\mathrm{T}$ cells, plasmacytoid dendritic cells, tumor-associated macrophages (TAMs), and MDSC $(3,5,6)$. Although the cellular interactions contributing to some of the pro-tumor factors present in the tumor microenvironment have been identified, the etiology of others remains unknown. 
Macrophages and MDSC are present within most solid tumors where they are major drivers of immune suppression and inflammation (3). We have previously reported that these cells participate in cross-talk with each other that results in increased MDSC production of IL-10 and decreased macrophage production of IL-12, thereby polarizing the immune system towards a pro-tumor type 2 environment $(7,8)$. Additional factors are also likely to be impacted by cross-talk between MDSC and macrophages, as well as by interactions with tumor cells. Therefore, we have investigated how tumor cells, macrophages, and MDSC interact with respect to IL-6, TNF $\alpha$, IL-10, and nitric oxide (NO). We have focused on these four molecules because they are chronically present in many solid tumors and play important roles in tumor progression. IL-6 promotes tumor progression by enhancing tumor cell development, growth, and metastasis, and by inhibiting apoptosis and enhancing tumor vascularization (9-11). TNF $\alpha$ causes DNA damage, inhibits apoptosis, and induces the production of matrix metalloproteases, cytokines, and chemokines that facilitate tumor cell invasion and metastasis (12). In contrast to IL-6 and TNF $\alpha$ which when chronically present are exclusively pro-tumor, NO can have both pro- and anti-tumor activity. When produced by M1-like macrophages, NO induces tumor cell apoptosis (13). However, when produced by MDSC, NO drives immune suppression (14). IL-10 has also been associated with both pro- and anti-tumor activity (15). Here we report that macrophages, MDSC, and tumor cells participate in a network of cross-talk resulting in differential production of IL-6, IL-10, TNF $\alpha$, and NO, suggesting that the interaction of these cells has the potential to significantly alter the inflammatory milieu within the tumor microenvironment. 


\section{Material and methods}

\section{Mice, tumor cells, tumor growth}

$\mathrm{BALB} / \mathrm{c}, \mathrm{C} 57 \mathrm{BL} / 6, \mathrm{BALB} / \mathrm{c}$ IL- $6^{-/-}$, and BALB/c IL-10 ${ }^{-/-}$mice were bred in the UMBC animal facility from stock obtained from The Jackson Laboratory (Bar Harbor, ME; C57BL/6 and BALB/c) or provided by Dr. Manfred Kopf (Zürich, Switzerland; IL-

$\left.6^{-/-}\right)$. BALB/c-derived 4T1 and TS/A mammary carcinomas, CT26 colon carcinoma, and C57BL/6-derived MC38 colon carcinoma were maintained as described (16). Mice were inoculated in the abdominal mammary gland with $100 \mu \mathrm{L}$ DMEM containing $7 \times 10^{3}$ (wild type and IL-10 $10^{-/}$mice) or $10^{5}$ (wild type and IL- $6^{-/-}$mice) $4 \mathrm{~T} 1$ cells, or $10^{6} \mathrm{TS} / \mathrm{A}$ cells; or s.c. in the flank with $5 \times 10^{5}, 1 \times 10^{5}$, or $1 \times 10^{4}$ CT26 cells. Primary tumors were measured as described (17). Survival time was recorded when mice became moribund and were euthanized. All animal procedures were approved by the UMBC Institutional Animal Care and Use Committee.

\section{Flow cytometry and antibodies}

Gr1-FITC, Gr1-APC, Ly6C-FITC, Ly6G-PB, CD11b-PE, CD11b-PB, F4/80APC, F4/80-PB, pSTAT3-PB, IL-6R-PE, IL-10R-PE mAbs, and rat IgG1-PE and IgG2bPE isotype were from BD Pharmingen (San Diego, CA) or BioLegend (San Diego, CA). Cells were stained for surface markers as described (18). For phosphoflow experiments, cells were stimulated with $50 \mathrm{ng} / \mathrm{mL}$ recombinant IL-10 (BioLegend) or supernatants from MDSC and macrophage co-cultures, fixed with Lyse/Fix Buffer (BD Bioscience), permeabilized with Perm Buffer III (BD Bioscience), and stained with antibodies diluted 
in Stain Buffer (BD Bioscience). Samples were analyzed on a Beckman/Coulter Cyan ADP flow cytometer using Summit software.

\section{$T$ cell proliferation assays}

$\mathrm{CD}^{+}$and $\mathrm{CD} 8^{+} \mathrm{T}$ cell proliferation assays were performed as described (18). Briefly, DO11.10 (ovalbumin peptide $323-339$-specific, I-A ${ }^{\mathrm{d}}$-restricted) or Clone4 (hemagglutinin peptide $518-526$-specific, $\mathrm{H}-2 \mathrm{~K}^{\mathrm{d}}$-restricted) splenocytes were cultured with their respective cognate peptides and irradiated blood MDSC from 4T1-bearing wild type, IL- $6^{--}$, or IL- $10^{-/-}$mice. Cultures were pulsed with ${ }^{3} \mathrm{H}$-thymidine on day 4 and harvested on day 5. Peptides were synthesized at the University of Maryland Baltimore (UMB) Biopolymer Core Facility.

\section{MDSC, macrophage, MDSC-macrophage-tumor cell cross-talk}

MDSC were isolated from the peripheral blood of 4T1 tumor-bearing mice (16). Peritoneal macrophages were prepared from tumor-free mice (8). MDSC and macrophages in all experiments were $>90 \% \mathrm{Gr}^{+} \mathrm{CD} 11 \mathrm{~b}^{+}$cells and $>95 \% \mathrm{CD} 11 \mathrm{~b}^{+} \mathrm{F} 4 / 80^{+}$ cells, respectively, as assessed by flow cytometry. MDSC and macrophage cross-talk experiments were performed as described (7) with the following modifications. 4T1, MC38, TS/A, or CT26 tumor cells ( $1 \times 10^{5}$ cells) were cultured with or without $7.5 \times 10^{5}$ MDSC and/or macrophages in $500 \mu \mathrm{L}$ macrophage media ( $5 \%$ fetal calf serum in DMEM, $1 \%$ penicillin-streptomycin, $1 \%$ glutamax, $0.1 \%$ gentamycin) for $16 \mathrm{hr}$ at $37^{\circ} \mathrm{C}$ with 100ng/mL LPS (Difco) and 20U/mL IFN $\gamma$ (R\&D Systems). In some experiments macrophages and/or MDSC were cultured with LPS and IFN $\gamma$ and either recombinant IL- 
6 (Biolegend), recombinant IL-10 (Biolegend), IL-10 that was denatured by boiling at $95^{\circ} \mathrm{C}$ for 15 minutes, or in the presence of neutralizing antibodies to IL-10 $(1 \mu \mathrm{g} / \mathrm{ml}$; clone JES5-2A5, eBioscience). Cells were harvested by scraping, and analyzed by flow cytometry. Supernatants were analyzed for IL-10, IL-6, and TNF $\alpha$ using ELISA kits (R\&D systems and eBioscience, San Diego, CA) per the manufacture's protocol, or by multiplex analysis in the UMB Cytokine Core Facility. NO production was quantified by Griess assay (18). Values were normalized between experiments using the following formulas:

Production of IL- 6 by MDSC or macrophages in response to tumor cells $=($ IL- 6 from wild type MDSC or macrophages with tumor cells)-(IL-6 from IL-6 ${ }^{-/-}$MDSC or macrophages with tumor cells).

$\%$ increase in IL-6 or NO by MDSC or macrophages in response to tumor cells $=\{[(\mathrm{IL}-6$ or NO from macrophages and MDSC \pm tumor cells)/(IL-6 or NO from macrophages)] $\mathrm{x}$ $100 \%\}-100 \%$.

$\%$ decrease in IL-6 or TNF $\alpha$ by macrophages in response to tumor cells and/or MDSC $=$ $1-[($ IL-6 or TNF $\alpha$ from macrophages \pm tumor cells)/(IL-6 or TNF $\alpha$ from wild type macrophages \pm tumor cells \pm MDSC)] x $100 \%$.

$\%$ increase in IL-10 by MDSC in response to macrophages $=\{[(\mathrm{IL}-10$ from macrophages + MDSC)/(IL-10 from MDSC)] x 100\% $-100 \%$. If IL-6 was not detected, then the lowest value detectable on the standard curve was utilized for the calculations. Macrophages and MDSC were stained with 5 $\mu \mathrm{M}$ CellTrace Violet (Life Technologies) and 4T1 tumor cells with $1 \mu \mathrm{M}$ CFSE (Life Technologies). MDSC or macrophages were cultured for 16 hours in macrophage media with 100ng/mL LPS and 20U/mL IFN $\gamma$ in a 6 
well dish at $3 \times 10^{6}$ cells/well $/ 2 \mathrm{~mL}$ either with or without $4 \times 10^{5} 4 \mathrm{~T} 1$ cells. Cells were then harvested using Detachin (Genlantis, San Diego, CA) and scraping, washed, and stained for Gr1, CD11b, and with 7AAD, and analyzed by flow cytometry.

\section{Ex vivo tumor cultures}

4T1, CT26, and TS/A tumors $>8 \mathrm{~mm}$ in diameter were surgically resected from euthanized mice and placed on sterile \#50 Whatman filter paper to remove excess liquid. The tumors were then transferred to $6 \mathrm{~cm}$ culture dishes, finely minced using a sterile scalpel, and the resulting pieces weighed. 4T1 and TS/A pieces were re-suspended in $5 \mathrm{~mL}$ of pre-warmed $4 \mathrm{~T} 1$ media (10\% fetal clone I in IMDM, $1 \%$ penicillin-streptomycin, $1 \%$ glutamax, $0.1 \%$ gentamycin) containing $100 \mathrm{ng} / \mathrm{mL}$ LPS and $20 \mathrm{U} / \mathrm{mL}$ IFN $\gamma$ for IL-10 studies, or without LPS and IFN $\gamma$ for IL-6 studies. Re-suspended tumor pieces were incubated for 16 hours at $37^{\circ} \mathrm{C}, 5 \% \mathrm{CO}_{2}$, and supernatants were analyzed for cytokine production by ELISA. Cytokine levels were normalized per gram of tumor tissue per $\mathrm{mL}$ of media using the following formula: cytokine production (normalized) $=$ cytokine $(\mathrm{pg} / \mathrm{mL}) \times[($ tumor weight $/ 1 \mathrm{~g}) \times 5 \mathrm{~mL}]$.

\section{Statistical Analyses}

Student's $t$-test and Tukey's Honestly Significant Difference (HSD) test were performed using Microsoft Excel 2013. Values denoted with different letters (e.g. a, b, c, etc.) are significantly different from each other; values with the same letter are not significantly different. Tumor growth and exogenous IL-10 data were analyzed using the Mann-Whitney test on the www.VassarStats.net website. Survival data were analyzed 
using the log-rank test from the Walter and Eliza Hall Institute of Medical Research Bioinformatics webpage (http://bioinf.wehi.edu.au/software/russell/logrank/). Values of $\mathrm{p}<0.05$ were considered statistically significant.

\section{Results}

\section{IL-6 and IL-10 promote tumor progression}

Increased levels of serum IL-6 are correlated with chronic inflammation, increased tumor burden, and poor prognosis in some human and mouse systems (19). IL6 also promotes MDSC-mediated inhibition of Th1 responses in mice (20). In contrast, IL-10 correlates with tumor progression in some systems, but with tumor regression in other systems $(15,21-25)$. To determine if IL-6 and/or IL-10 contribute to progression of the 4T1 mammary carcinoma or CT26 colon carcinoma, we inoculated syngeneic wild type, IL-6 ${ }^{-1-}$, and IL-10 $0^{-/-}$mice with 4T1 (Figure 1A) or CT26 (Figure 1B) tumor cells and followed the mice for tumor onset, growth, and engraftment. In the absence of hostproduced IL-6, 4T1 tumor progression was delayed and survival time was increased. IL$10^{-/-}$mice showed a similar, although less dramatic delay in tumor progression and extension of survival time. $4 \mathrm{~T} 1$ tumor engraftment in wild type BALB/c and IL-10-- mice was $90-100 \%$, whereas only $40 \%$ of IL- $6^{-/-}$mice developed tumor. Tumor progression was also delayed and survival time increased in IL- $6^{-1-}$ mice with CT26 tumors. In contrast, IL-10 $0^{-/-}$mice inoculated with $5 \times 10^{5} \mathrm{CT} 26$ tumor cells had similar tumor progression, survival time, and percent engraftment as wild type mice. Tumor progression, survival time, and engraftment were also similar in wild type and IL-10 ${ }^{-/-}$ 
BALB/c mice inoculated with $1 \times 10^{5}$ or $1 \times 10^{4}$ cells (Supplemental Figure 1A). These results demonstrate that stromal cell-derived IL-6 and IL-10 facilitate progression of 4T1 and CT26 tumors in their syngeneic hosts.

\section{MDSC production of IL-10 decreases macrophage IL-6 and TNFa, and increases} NO. IL-6 indirectly regulates MDSC production of IL-10.

We have previously shown that MDSC production of IL-10 is enhanced by crosstalk with macrophages and polarizes macrophages towards a tumor-promoting phenotype by inhibiting macrophage production of IL-12 $(7,8)$. To determine if IL-10 produced by MDSC impacts the production of additional pro-inflammatory mediators, we co-cultured $\mathrm{CD} 11 \mathrm{~b}^{+} \mathrm{F} 4 / 80^{+}$peritoneal macrophages and $4 \mathrm{~T} 1$-induced $\mathrm{Gr}^{+} \mathrm{CD} 11 \mathrm{~b}^{+}$immune suppressive MDSC (Figure 2A), and assayed the supernatants for IL-10 and the proinflammatory cytokine IL-6 (Figure 2B). Consistent with our previous reports, production of IL-10 was significantly increased in the presence of macrophages (average increase in IL-10 of $116 \% \pm 19.4 \%$ for 30 experiments). IL-10 was produced exclusively by MDSC since macrophage cultures containing IL-10-- MDSC produced no IL-10. In the same co-cultures, macrophages were the sole producers of IL-6, and MDSC decreased macrophage IL-6 (average decrease in IL-6 of $24 \% \pm 3.8 \%$ for 30 experiments).

To determine if IL-6 regulates MDSC production of IL-10, we co-cultured wild type or IL-6 $6^{-/-}$macrophages with wild type or IL-6 ${ }^{-/-}$MDSC (Figure 2C). IL-6 $6^{-/-}$MDSC produced significantly more IL-10 than wild type MDSC. Macrophage co-cultures with IL-6 ${ }^{-/-}$MDSC had significantly more IL-10 than co-cultures with wild type MDSC. 
Macrophage IL-6 had no effect on MDSC IL-10, since wild type MDSC co-cultured with either wild type or IL-6 ${ }^{-/-}$macrophages produced similar amounts of IL-10. The lack of a direct effect by IL-6 on MDSC IL-10 was confirmed by incubation of MDSC with exogenous IL-6 (Supplemental Figure 1B). These results indicate that MDSC do not produce IL-6 in the co-culture setting; however, their development in vivo in the presence of IL-6 down-regulates their production of IL-10.

To determine if IL-10 produced by MDSC decreased macrophage IL-6, or regulated other molecules characteristic of tumor-rejecting M1 macrophages, wild type or IL-10 ${ }^{-/}$MDSC were co-cultured with wild type macrophages (Figure 2D). There was no decrease in IL-6 in the presence of IL-10 ${ }^{-/-}$MDSC, suggesting that IL-10 from wild type MDSC reduced macrophage IL-6. To confirm the role of IL-10, neutralizing antibodies to IL-10 were added to MDSC-macrophage co-cultures. Since previous studies demonstrated that MDSC IL-10 also decreases macrophage IL-12 (8), IL-12 levels served as a positive control (Figure 2E). IL-10 neutralizing antibodies reduced the MDSCmediated decrease of both IL-6 and IL-12. Thus, a feedback loop exists between macrophages and MDSC in which macrophages increase MDSC production of IL-10, and MDSC IL-10 regulates macrophage synthesis of IL-6.

We also assessed the role of MDSC IL-10 on macrophage NO and TNF $\alpha$ production (Figure 2D). MDSC IL-10 decreased TNF $\alpha$ in the co-cultures; however, this decrease was minimal. In contrast, macrophage production of NO was increased by coculture with MDSC. The increase was predominantly due to MDSC IL-10 since only a minimal increase in NO was observed in the presence of IL-10 ${ }^{-/-}$MDSC. 
To further confirm that IL-10 regulated macrophage production of IL-6 and NO, and macrophage and MDSC production of TNF $\alpha$, macrophages or MDSC were cultured in the presence of exogenous IL-10, and culture supernatants were assessed for TNF $\alpha$, IL-6, and NO (Figure 2F). Exogenous IL-10 reduced MDSC and macrophage TNFa, and macrophage IL-6, but increased macrophage NO. Since STAT3 is activated by signaling through the IL-10 receptor, macrophages were cultured with exogenous IL-10, or with supernatants from MDSC-macrophage co-cultures, and subsequently stained for phosphorylated STAT3 (Figure 2G). STAT3 was phosphorylated under both conditions, further confirming the regulatory role of IL-10 produced by MDSC.

MDSC and macrophages express IL-6R and IL-10R, respectively (Figure 2H) so these cells have the potential to respond directly to these cytokines. The results of Figure 2F suggest that IL-10 directly impacts macrophages. However, IL-10-deficiency and IL6-deficiency could also cause other changes in MDSC and/or macrophages, so that the effects are only indirectly mediated by IL-10 or IL-6. To distinguish these possibilities, we compared cytokine/chemokine production by wild type, IL-10 $10^{-/}$, and IL- $6^{-/-}$MDSC to determine if gene deficiency impacts MDSC phenotype (Supplemental Table 1). TGFß3, GM-CSF, IL-4, IL-13, and IL-23 were not detectable in wild type MDSC. TGF$\beta 2$, IL-1 $\beta$, CCL2, and VEGF production were similar for wild type, IL-10 ${ }^{-1}$, and IL-6 ${ }^{-1-}$ MDSC. TGF- $\beta 1$ trended higher in IL- $10^{-/-}$and IL- $6^{-/-}$MDSC, and MIP- $1 \alpha$ trended lower in IL-10 $0^{-/-}$and IL-6 $6^{-/-}$MDSC as compared to wild type MDSC. These results suggest that IL-10-deficiency and IL-6-deficiency may alter the phenotype of MDSC. 
These results together with our earlier studies on $\operatorname{IL}-12(7,8)$ demonstrate that MDSC production of IL-10 increases some M2-like characteristics of macrophages (ie. IL-12 $\left.{ }^{\text {low }} \mathrm{IL}-6^{\text {low }}\right)$, but also increases some M1-like properties $\left(\mathrm{NO}^{\text {high }}\right)$.

\section{Other cytokines are also impacted by interactions between MDSC and macrophages}

In addition to IL-10, TNF $\alpha$, IL-12, NO, and IL-6, other immune regulatory molecules are present in solid tumors. Of particular note are cytokines that drive effector and regulatory T cells (e.g. IL-23, IL-27, IL-4, and IL-13), growth factors that regulate neovascularization (e.g. VEGF) and myeloid cell differentiation (e.g. GM-CSF), proinflammatory mediators (e.g. IL-1 $\beta$ ), and immune suppressive molecules (e.g. TGF- $\beta$ ). To determine if any of these molecules are affected by cross-talk between MDSC and macrophages, supernatants from co-cultures of 4T1-induced wild type MDSC and wild type BALB/c macrophages were assayed by multiplex analysis (Supplemental Table 1). Neither MDSC nor macrophages produced TGF- $\beta 3$, GM-CSF, IL-4, IL-13, or IL-23, while both cell types produced TGF- $\beta 1$, TGF- $\beta 2$, IL- $1 \beta$, CCL2, MIP- $1 \alpha$, and VEGF. Cocultures utilizing wild type MDSC reduced the production of TGF- $\beta 1$, TGF- $\beta 2$, and MIP$1 \alpha$, and modestly increased the production of VEGF. Co-cultures of wild type macrophages with IL-10 $0^{-/-}$or IL-6 ${ }^{-/-}$MDSC displayed similar trends except for CCL2, where we observed a decrease in CCL2 production.

\section{Tumor cells increase MDSC production of IL-6 and vice versa}

Tumor cells produce pro-inflammatory mediators and therefore may contribute to the polarization of myeloid cells in the tumor microenvironment. To assess if there is 
cross-talk between MDSC and tumor cells, 4T1, CT26, TS/A, or MC38 murine tumor cells were cultured by themselves or co-cultured with MDSC (Figure 3). When cultured alone, 4T1 and CT26 cells produced IL-6, and TS/A, MC38, and MDSC produced no detectable IL-6. Cultures containing wild type MDSC plus 4T1, CT26, TS/A, or MC38 tumor cells contained more IL-6 than cultures of tumor cells alone, while cultures of 4T1, CT26, and TS/A tumor cells plus IL-6-deficient MDSC produced intermediate levels of IL-6. Cultures of MC38 tumor cells plus IL-6 ${ }^{-/-}$MDSC produced very low levels of IL-6. Increases in IL-6 production in the presence of IL- $6^{-/-}$MDSC indicate that in vitro, MDSC enhanced tumor cell production of IL-6. However, since IL-6 levels in co-cultures of wild type MDSC plus tumor cells were even higher than IL-6 production in co-cultures with IL-6-deficient MDSC, MDSC may also be induced by tumor cells to synthesize IL6. Interestingly, the MDSC, but not the tumor cells, proliferated during the overnight culture (Supplemental Figure 1C), so the increase in IL-6 in this setting could be due to higher numbers of MDSC. In contrast, tumor cells did not impact MDSC production of TNFa, IL-12, or IL-10 (Supplemental Figure 2). These results demonstrate that in vitro, reciprocal cross-talk between MDSC and most tumor cells increases IL-6 production and there is no cross-talk between MDSC and tumor cells with respect to IL-10, IL-12, or TNF $\alpha$.

\section{Tumor cells increase macrophage IL-6 and NO, and decrease macrophage TNFa}

To assess if there is cross-talk between macrophages and tumor cells, 4T1, CT26, TS/A, or MC38 tumor cells were cultured with macrophages and the culture supernatants assayed for IL-6, NO, and TNFa (Figure 4). All four tumor lines increased macrophage 
production of IL-6. Macrophages also increased IL-6 produced by 4T1, CT26 and TS/A tumor cells because cultures containing tumor cells plus IL- $6^{-/-}$macrophages produced more IL-6 than tumor cells alone. In co-cultures of wildtype or IL- $6^{-/-}$macrophages with 4T1, TS/A, or MC38 tumor cells, macrophages were the dominant produces of IL-6 (Figure 4A). In contrast, tumor cells were the dominant producers of IL-6 in cultures of macrophages plus CT26 tumor cells, indicating that some tumor cells have a greater response to macrophages. Cross-talk-induced increases in IL-6 ranged from $43 \%$ to $230 \%$. These results indicate that tumor cell production of IL-6 is differentially affected by macrophages and that macrophages produce IL-6 in response to tumor cells.

4T1, CT26, and TS/A cells also increased macrophage production of NO, and increases in NO ranged from $36 \%$ to $72 \%$ (Figure 4B). In contrast, macrophage production of TNF $\alpha$ was significantly decreased in the presence of the four tumors because cultures of macrophages plus tumor cells produced significantly less TNF $\alpha$ as compared to macrophages cultured alone. Tumor-cell mediated decreases in TNF $\alpha$ ranged from $24 \%$ to $53 \%$ (Figure 4C). Macrophage production of IL-10 and IL-12 was not affected by tumor cells (Supplemental Figure 2). Increases in macrophage NO and IL-6 were due to increased production by individual macrophages since the macrophages did not proliferate during the overnight culture period (Supplemental Figure 1C). These results show that macrophages and tumor cells participate in cross-talk with each other resulting in differential production of pro-inflammatory mediators which are characteristic of both $\mathrm{M} 1\left(\mathrm{NO}^{\text {hi }}\right)$ and $\mathrm{M} 2\left(\mathrm{TNF} \alpha^{\text {low }}\right)$ macrophages. 


\section{MDSC prevent most tumor cells from increasing macrophage IL-6}

Since MDSC and macrophages are present in the tumor microenvironment, we next tested if MDSC alter cross-talk between tumor cells and macrophages. MDSC, macrophages, and/or tumor cells were co-cultured and IL-6 levels were assessed (Figure 5A). Cultures containing 4T1, TS/A, or MC38 tumor cells plus MDSC and macrophages produced less IL-6 than cultures without MDSC. MDSC -mediated decreases of IL-6 ranged from $0 \%$ to $37 \%$. In contrast, MDSC did not decrease IL-6 in cultures of macrophages and CT26 tumor cells. These results demonstrate that in the presence of most tumors, MDSC modestly reduce macrophage IL-6.

\section{MDSC increase macrophage $\mathrm{NO}$ in the presence of tumor cells}

To determine if MDSC affect the tumor-driven increase in macrophage NO, tumor cells, macrophages, and MDSC were co-cultured (Figure 5B). Cultures of 4T1 or CT26 tumor cells with macrophages and MDSC contained more NO than cultures without MDSC. MDSC-mediated increases in NO ranged from $0 \%$ to $30 \%$. In contrast, TNF $\alpha$, IL-10, and IL-12 were not affected by MDSC (Supplemental Figure 2). These results indicate that MDSC alter the dynamic of tumor cell and macrophage cross-talk by enhancing NO production.

\section{Stromal cells are the dominant producers of IL-6 and IL-10 in the tumor microenvironment}

Our in vitro findings suggest that tumor-infiltrating cells and not tumor cells are the dominant producers of IL- 6 and IL-10. To determine if this in vitro finding occurs in 
vivo, we harvested 4T1, CT26, and TS/A tumors from wild type, IL-6 $6^{-1-}$, and IL-10 $10^{-1-}$ mice, and assayed the tumors for IL-6 and IL-10 (Figure 6). Tumors in all wild type mice contained both IL- 6 and IL-10, whereas all tumors from IL-10 $10^{-/}$mice contained very little or no IL-10. With the exception of one mouse with CT26 tumor, tumors from IL-6 $6^{-1-}$ mice did not have IL-6. Isolated tumors did not contain detectable levels of TNF $\alpha$ or NO. These results demonstrate that in vivo in the tumor microenvironment stromal cells and not tumor cells are the dominant sources of IL-6 and IL-10.

\section{Discussion}

Solid tumors include multiple, diverse host cells that contribute to an inflammatory tumor microenvironment and facilitate tumor progression. Because macrophages and MDSC are present in most solid tumors, we have examined the interplay of these cells to determine if and how their interactions may influence the intratumor environment. The studies reported here on IL-6, IL-10, TNF $\alpha$, and NO, plus our previous reports on IL-12 address some of the most common molecules produced by MDSC and macrophages that contribute to tumor progression. Our findings are summarized in Figure 7A. Collectively, our results indicate that the levels of IL-6, IL-10, IL-12, TNF $\alpha$, and NO are modulated by interactions between MDSC, macrophages, and tumor cells. MDSC induce some M2 macrophage characteristics (IL-6 $6^{\text {low }} \mathrm{IL}-$ $\left.12^{\text {low }} \mathrm{TNF}^{\text {low }}\right)$, but simultaneously induce NO which is characteristic of M1 macrophages. These apparently opposing activities are both regulated by MDSC production of IL-10. Tumor cells also regulate macrophage expression of molecules characteristic of both M1 (IL- $\left.6^{\text {hi }} \mathrm{NO}^{\text {hi }}\right)$ and M2 $\left(\mathrm{TNF}^{\text {low }}\right)$ phenotypes, while tumor cells 
and macrophages enhance MDSC production of IL-6 and IL-10, respectively. Since stromal cells are the dominant producers in vivo of several of these cytokines, the complex pattern of cross-talk between MDSC, macrophages, and tumor cells is likely to have profound effects on tumor progression.

Nitric oxide is an important effector molecule that is differentially impacted by IL-10 and either promotes or inhibits tumor progression depending on the tumor model. NO is produced by endothelial nitric oxide synthase (NOS3) and inducible nitric oxide synthase (NOS2) which are up-regulated (26) and down-regulated (27), respectively, by macrophage-produced IL-10. Both pro and anti-tumor roles have been attributed to NO/NOS2 in multiple tumor systems (Supplemental Table 2). It is likely that the apparent conflicting effects of NO are due to many variables, including, but not limited to the production of NO by different types of cells, the location of the producer cells, neighboring cells that might be altered by the released NO, and the concentration of NO. Due to the complexity of NO on tumor progression and the presence of multiple cell types in the tumor micro-environment that may participate in cross-talk, elucidating the role of NO in tumor progression will be challenging.

IL-6 is a pivotal cytokine that directly promotes tumor progression by enhancing tumor cell development, growth, metastasis, vascularization, and inhibiting apoptosis (911). MDSC were reported to be a primary producer of IL-6 in the tumor microenvironment (20). This observation is consistent with our finding that stromal cells and not tumor cells are the major producer of IL-6 in vivo, and that tumor cells drive MDSC IL-6 production. IL-6 also enhances MDSC accumulation and suppressive activity (28-30) and decreases MDSC production of IL-10, an anti-inflammatory cytokine 
(31). Therefore, positive feedback between MDSC and tumor cells will potentially maintain chronic inflammation and promote tumor progression through the cycle shown in Figure 7B.

Both pro- and anti-tumor roles have been attributed to IL-10. It down-regulates numerous immune modulatory molecules which are essential for an anti-tumor immune response and is considered an anti-inflammatory cytokine $(15,31)$. For example, IL-10 impairs antigen presentation by dendritic cells and macrophages by down-regulating expression of MHC class II, CD80, and CD86. IL-10 also decreases production of IFN $\gamma$ and IL-12, cytokines that are characteristic of and facilitate the development of type I anti-tumor effector and helper cells, and IL-10 over-expressing tumor cells have increased growth rates in vivo (32). In cancer patients, secretion of IL-10 from basal or squamous cell carcinoma cells prevents in vitro lysis of tumor cells by tumor-infiltrating lymphocytes. In vitro, pretreatment of tumor cells (e.g. melanoma, lymphoma) with IL10 confers resistance to CTL-mediated lysis by decreasing expression of transporter associated with antigen processing (TAP) -1 and -2 and subsequent surface expression of MHC I. IL-10 also contributes to tumor progression by enhancing angiogenesis and tumor cell proliferation. Since MDSC IL-10 is enhanced by macrophage cross-talk, and IL-10 is produced predominantly by tumor-infiltrating stromal cells, cross-talk by macrophages and MDSC is most likely a source of IL-10 in the tumor microenvironment.

However, IL-10 has also been linked to enhancing anti-tumor immunity (15). For example, the reduction in MHC I by IL-10 renders tumor cells more susceptible to NKmediated killing, and a tumor cell-based glioma vaccine induced more effective antitumor immunity in wild type mice than in IL-10-- mice. IL-10 also directly activated 
tumor-resident $\mathrm{CD} 8^{+} \mathrm{T}$ cells and facilitated tumor rejection of PDV6 squamous carcinoma (23), and served as an adjuvant in immunotherapy. Treatment of mice with pegylated IL-10, a form of IL-10 that has increased serum half-life, induces IFN $\gamma$ and granzyme-B production by tumor-infiltrating $\mathrm{CD} 8^{+} \mathrm{T}$ cells in an MMTV tumor model (24). IL-10 also inhibited tumorigenesis in mice with colon carcinoma and patients with B cell lymphoma $(21,22)$. Ablation of IL-10 from $\mathrm{CD}^{+} \mathrm{T}$ cells enhanced tumor burden in $\mathrm{APC}^{\Delta 468}$ mice (33), while IL-10 ${ }^{-/-}$mice bearing MC38 tumors displayed increased tumor growth, metastasis, MDSC accumulation and enhanced susceptibility to chemical carcinogenesis (34). Therefore, as reported in the literature and shown in this report, the role of IL-10 in the promotion of tumor progression is dependent on the tumor model. STAT3 is activated by both IL-6 and IL-10; however, the two cytokines can result in different biological effects due to the complexity of the STAT3 pathway (35). There are $1.3 \times 10^{6}$ potential binding sites for STAT3 in the mouse genome (36); however, STAT3 only binds a few thousand sites in a given cell type (37). STAT3 is a pleiotropic transcription factor which regulates target genes by acting in conjunction with a variety of transcriptional co-activators. The expression of these co-activators is dependent on the cell type and signaling events that occur in a cell's lifetime. Many of these co-activators are pre-bound to STAT3 target sites (reviewed in (37). Therefore, a cell's phenotype following STAT3 signaling depends on it's previous history with respect to STAT3 activation. The tumor microenvironment is a complex milieu, so differential expression of transcriptional co-activators is likely. Since MDSC-macrophage-tumor cell cross-talk involves activation of STAT3 via both IL-6 and IL-10, and the relative amounts of these 
cytokines differ depending on the type of tumor, cross-talk is likely to contribute to the differential effects of IL-10 on tumor progression.

In addition to the cells examined here, other stromal cells also contribute to inflammation within the tumor microenvironment through their cross-talk with MDSC $(38,39)$. However, MDSC and macrophages are present at significant levels in most solid tumors, and therefore their contributions to the inflammatory milieu are likely to be important. 


\section{Figures}

Figure 1
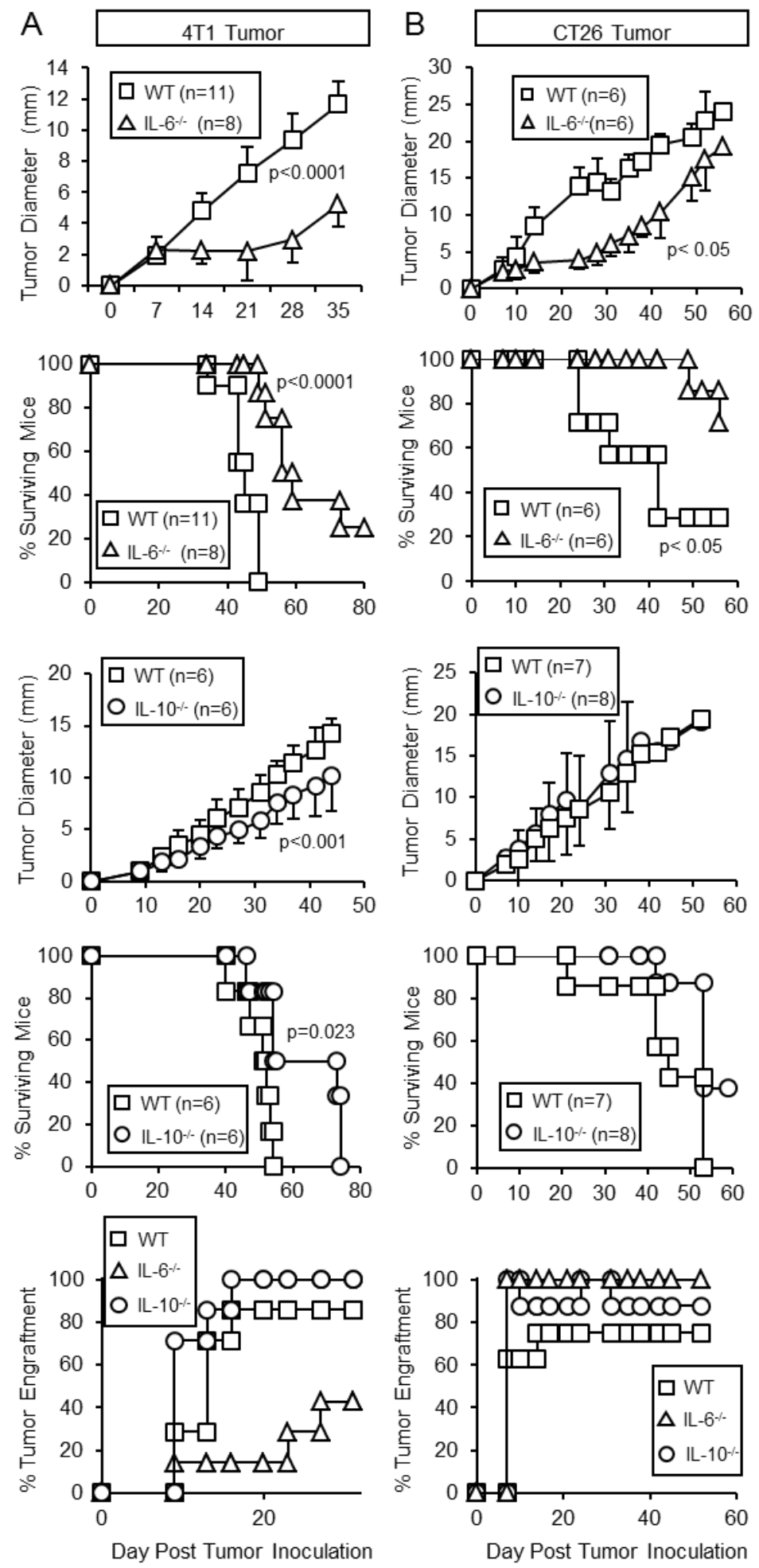
Figure 1: IL-6 and IL-10 produced by host cells enhance primary tumor growth and decrease survival time. Wild type, IL-6-/-, and IL-10-/- BALB/c mice were inoculated with (A) 4T1 or (B) CT26 tumor cells and monitored for tumor diameter, survival, and tumor incidence. Mice in the wild type vs. IL-6-/- graphs and wild type vs. IL-10-/graphs (tumor diameter and \% survival) were inoculated with 1x105 and 7000 4T1 cells, respectively. Mice in the engraftment graph were inoculated with 1x105 4T1 cells. All CT26 inoculations were 5x105 cells. Statistical significance was tested by Mann-Whitney (tumor growth) or log-rank test (survival). Data are pooled from three independent experiments. 
Figure 2

A
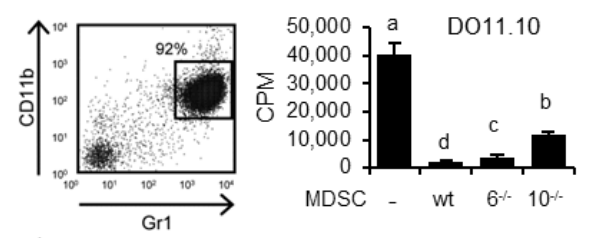

B
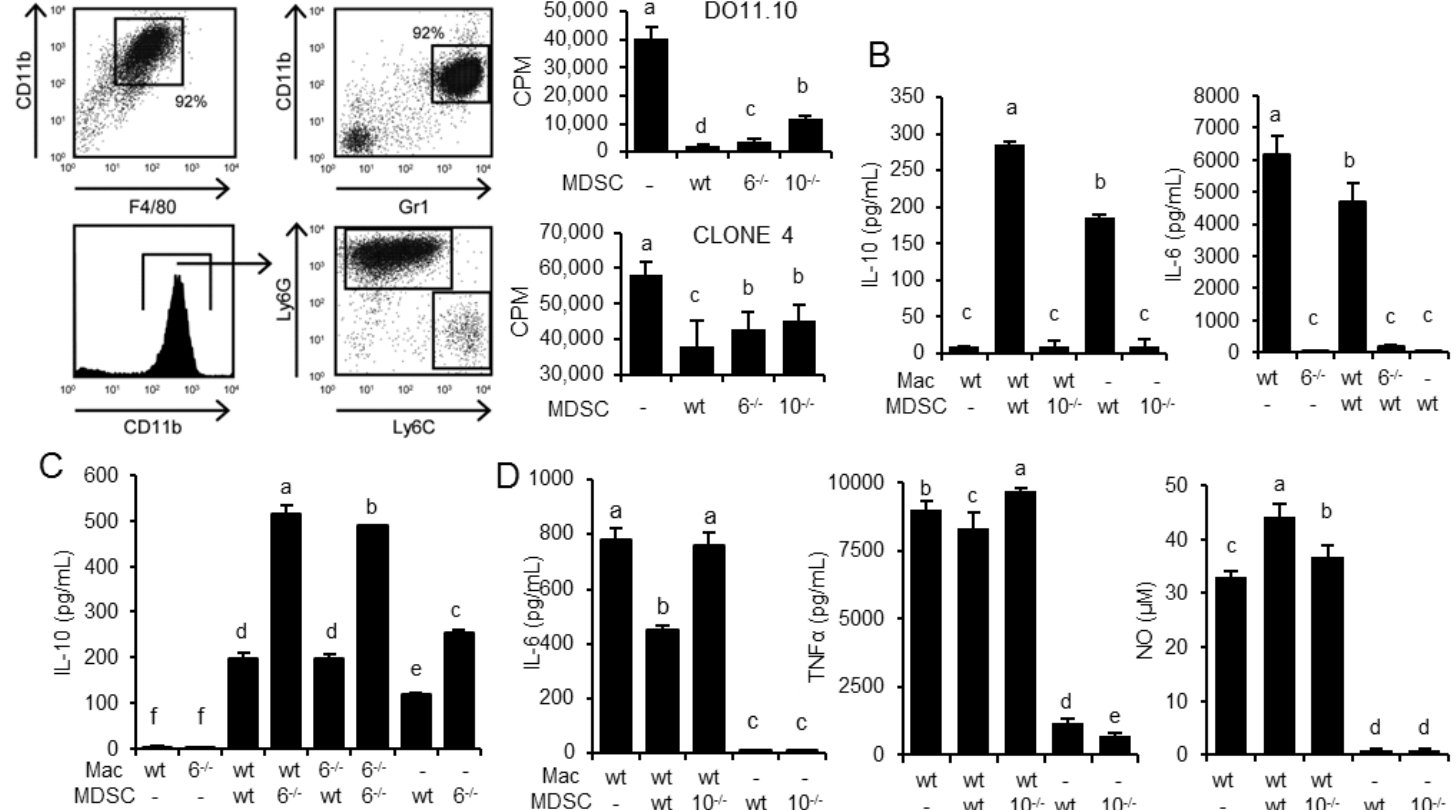

MDSC

MDSC - wt $10^{-}$wt 10

wt wt wt

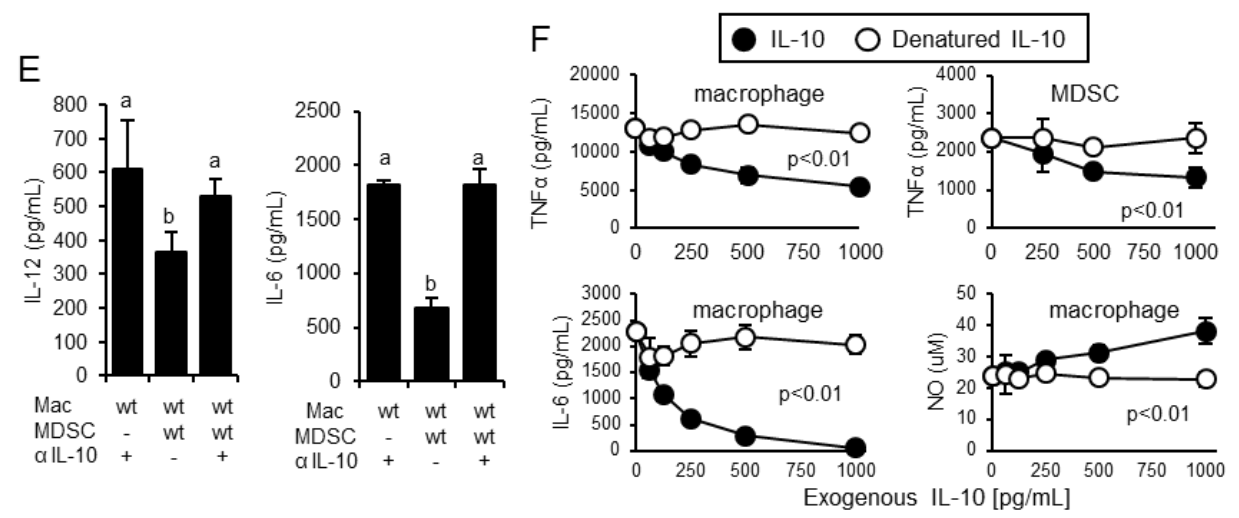

G
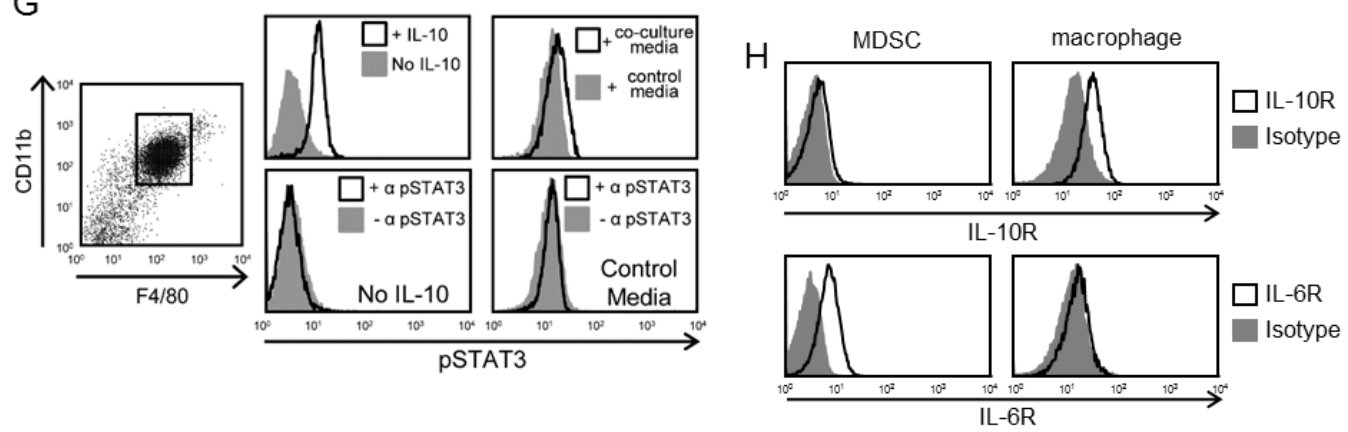
Figure 2: Cross-talk between MDSC and macrophages regulates production of IL-10, IL6 and nitric oxide. (A) Peritoneal macrophages from healthy mice and MDSC from tumor-bearing mice were stained with mAbs to CD11b, F4/80, Gr1, Ly6C, and/or Ly6G and analyzed by flow cytometry. MDSC from wild type, IL-10-/-, and IL-6-/- BALB/c mice with 4T1-tumors were assayed for their ability to suppress the antigen-driven activation of peptide-specific, MHC-restricted, transgenic CD4+ (DO11.10) and CD8+ (Clone4) T cells. (B-D) 4T1-induced MDSC and peritoneal macrophages from wild type, IL-10-/-, or IL-6-/- BALB/c mice were co-cultured and supernatants were assayed for IL10, IL-6, and NO. (B) Macrophages enhance MDSC IL-10 and MDSC decrease macrophage IL-6. (C) IL-6 decreases MDSC IL-10. (D) IL-10 production by MDSC decreases macrophage IL-6 and TNF $\alpha$, and increases macrophage NO. (E) Neutralizing antibodies to IL-10 prevent the down-regulation of macrophage IL-6 and IL-12. (F) Exogenous IL-10 decreases MDSC and macrophage TNF $\alpha$, decreases macrophage IL-6, and enhances macrophage NO. Macrophages or MDSC were cultured with either IL-10 or denatured IL-10. (G) Macrophages activate STAT3 in response to IL-10. Macrophages (left-hand panel) were cultured for $5 \mathrm{~min}$ in the presence or absence of exogenous IL-10 (middle two panels) or with supernatants (media) from MDSC-macrophage co-cultures (right-hand two panels), subsequently fixed and permeabilized, and then stained for F4/80, CD11b, and pSTAT3. (H) Macrophages and MDSC express the receptors for IL10 and IL-6, respectively. Data for panels A-H are from one of 2, 30, 3, 5, 4, 3, 2, and 2 experiments, respectively. Statistical significance for panels A-E and F were determined by Tukey's HSD test and the Mann-Whitney test, respectively; panels E. Different lower case letters above each value indicate that those values are statistically significantly 
different; values that share the same lower case letter are not statistically significantly different. 


\section{Figure 3}

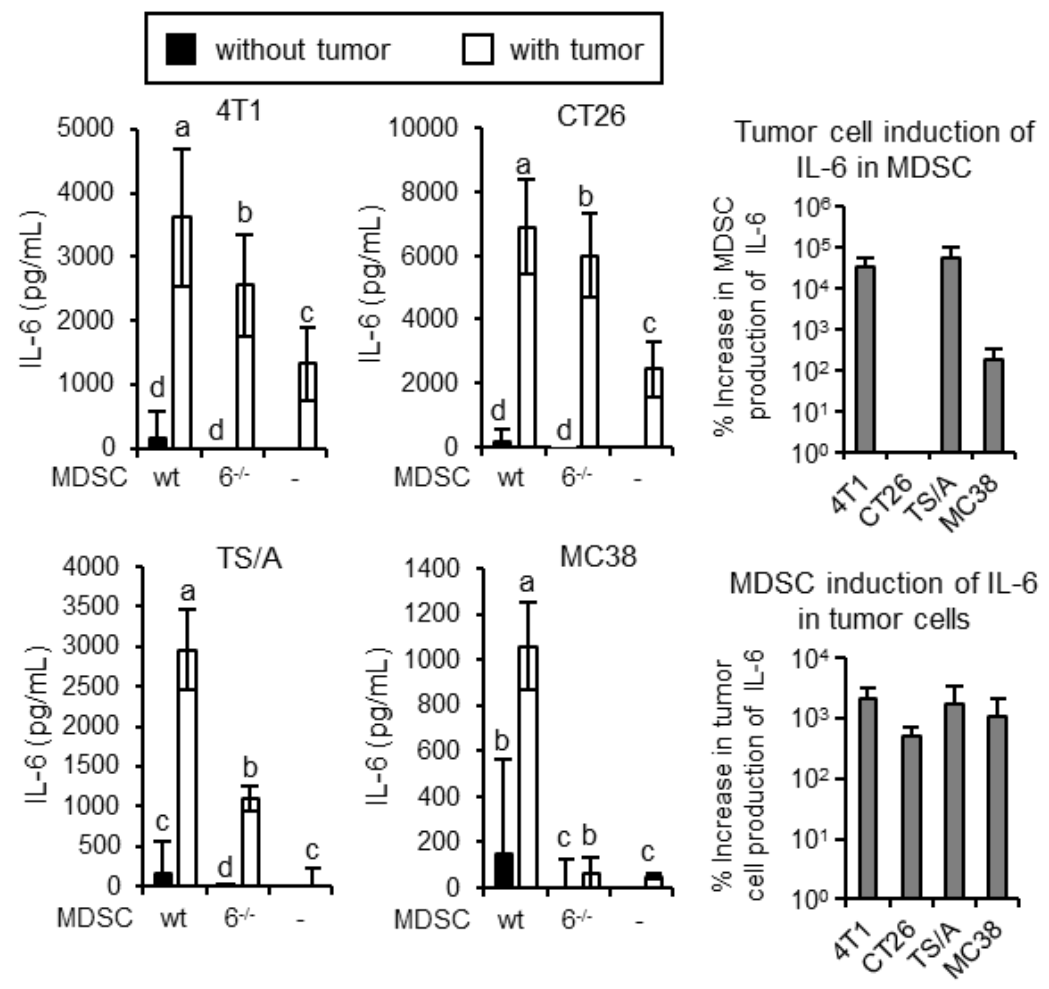

Figure 3: Tumor cells induce MDSC to produce IL-6 and vice versa. Wild type or IL-6-/4T1-induced MDSC were cultured with or without 4T1, CT26, TS/A, or MC38 tumor cells and the supernatants were assayed for IL- 6 by ELISA. One of three independent experiments (left-hand four graphs); average percent increase of three independent experiments comparing tumor cells with IL-6-/- and wild type MDSC (right-hand two graphs). Statistical significance for the independent experiments was determined by Tukey's HSD test. 
Figure 4

without tumor $\square$ with tumor

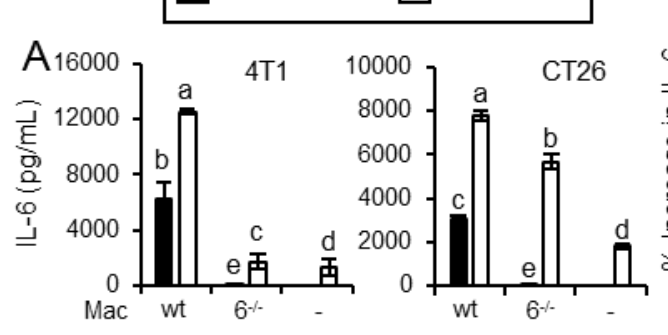

Tumor cell induction of 200 IL-6 in macrophages i 160.

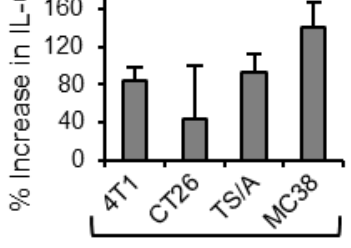

+ Macrophages
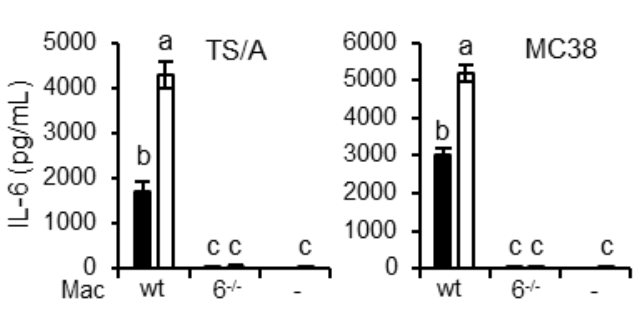

Macrophage induction

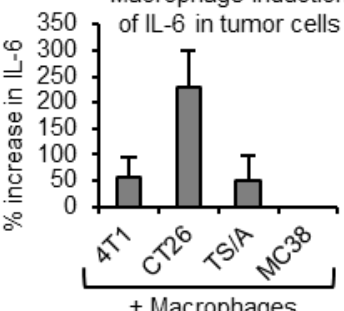

B
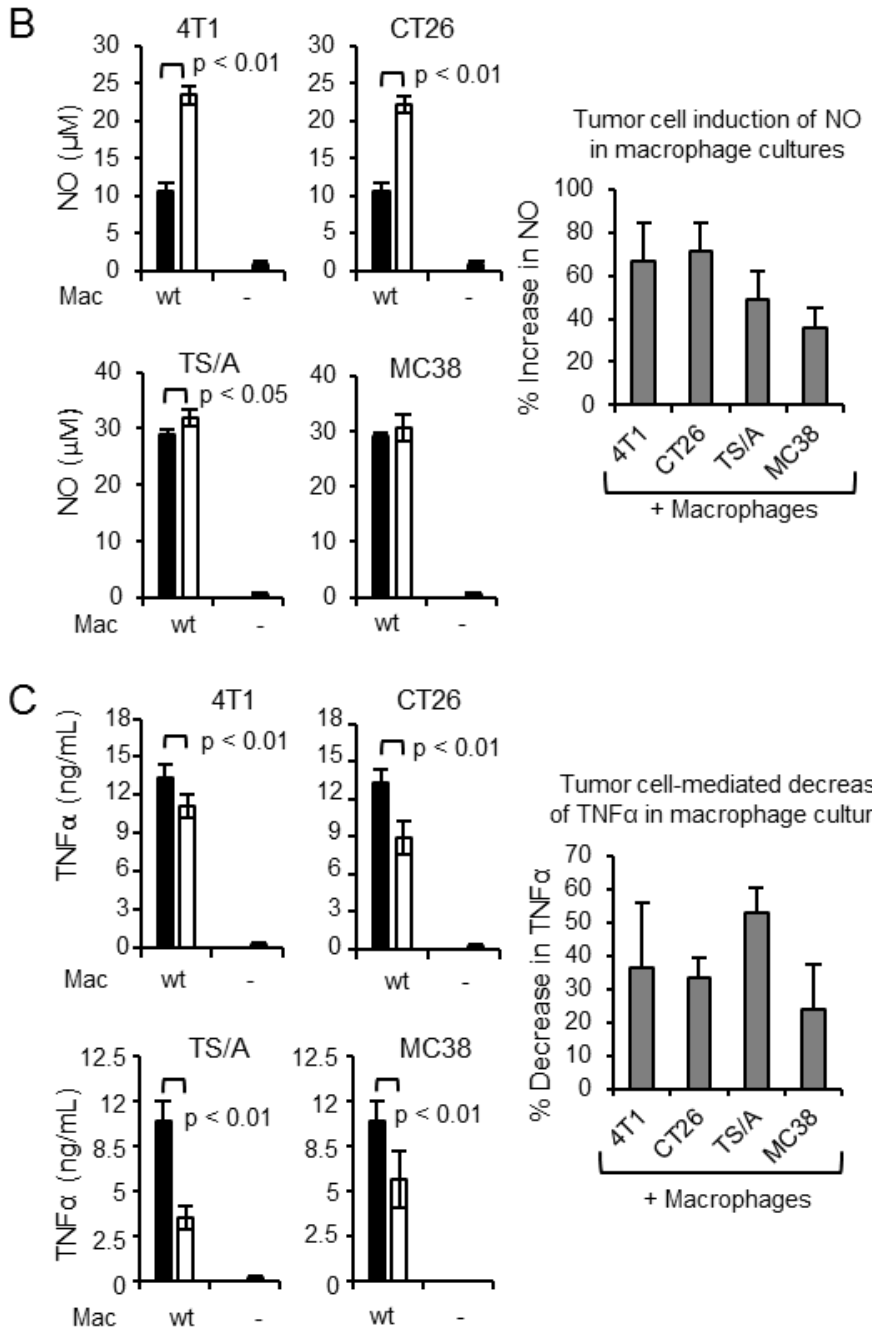

Tumor cell-mediated decrease of TNFa in macrophage cultures

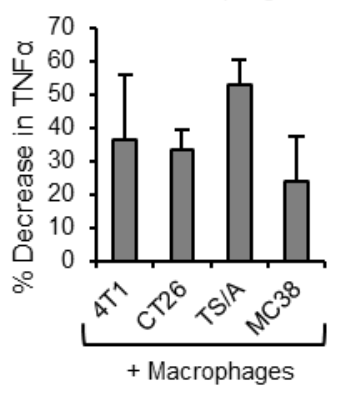


Figure 4: Tumor cells induce macrophages to produce IL-6 and NO, but decrease macrophage TNF $\alpha$. Wild type or IL-6 ${ }^{-/-}$macrophages were cultured with or without 4T1, CT26, TS/A, or MC38 tumor cells and the supernatants were assayed for (A) IL-6, (B) NO, or (C) TNF $\alpha$. Left-hand graphs show representative data from one of four, three, and four independent experiments, respectively. Right-hand graphs show average percent change of pooled data from all experiments. (A) Comparison of tumor cells with IL- $6^{-/-}$ and wild type macrophages. (B and C) Comparison of tumor cells with wild type macrophages. Statistical significance for panels A, B and C was determined by $t$-test. 


\section{Figure 5}

without tumor $\square$ with tumor
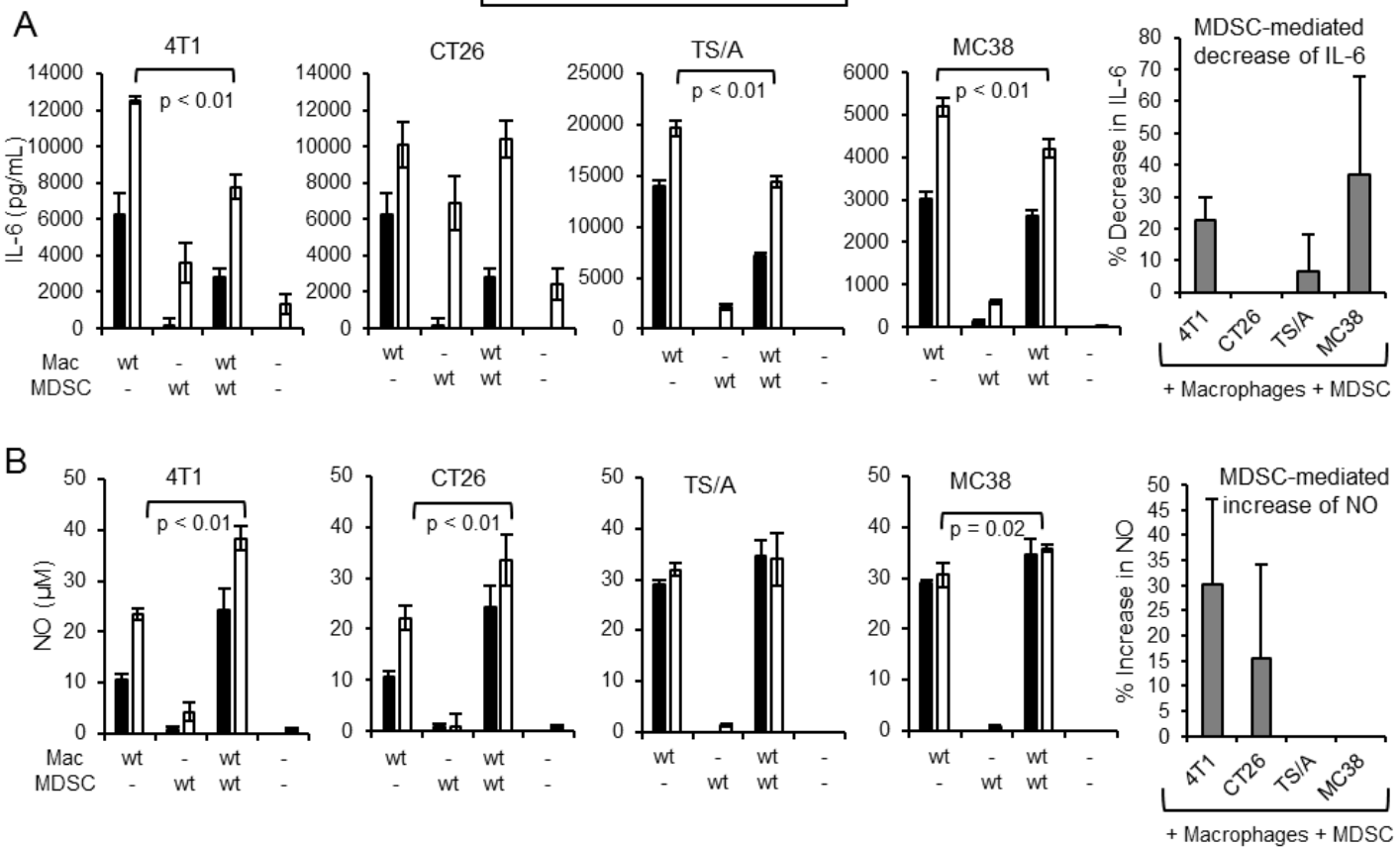

Figure 5: MDSC decrease tumor cell-mediated enhancement of IL-6, and increase tumor cell-mediated enhancement of macrophage NO. Wild type macrophages were cultured with or without 4T1-induced MDSC and/or 4T1, CT26, TS/A, or MC38 tumor cells. Supernatants were assayed for (A) IL-6 and (B) NO. Left-hand four graphs of panels A and $\mathrm{B}$ are one of three independent experiments. Right-hand graphs show the average of the three independent experiments. Statistical significance for panels A and B was determined by $t$-test. 


\section{Figure 6}
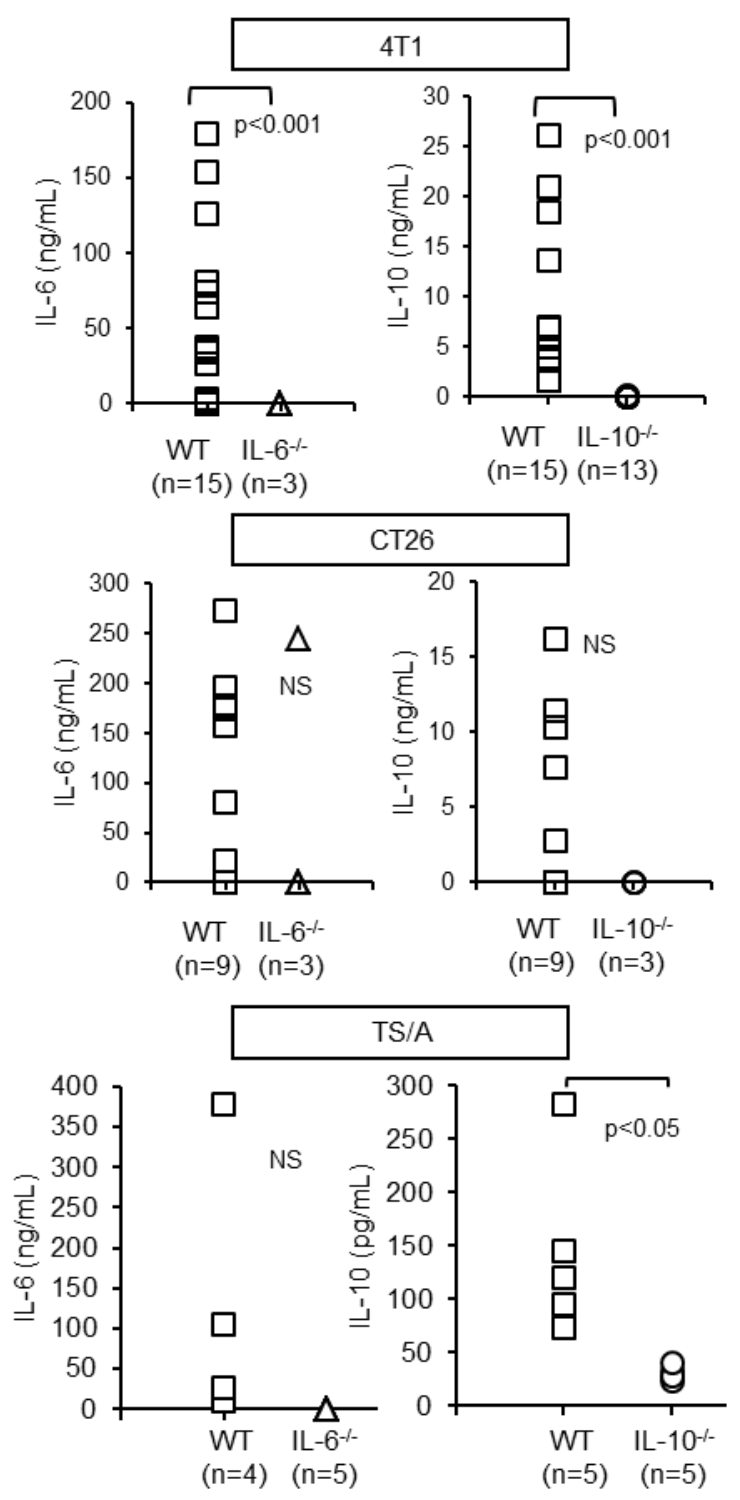

Figure 6: Host cells are the dominant producers of IL-6 and IL-10 in the tumor microenvironment. Eight to $10 \mathrm{~mm}$ diameter (A) 4T1, (B) CT26, and (C) TS/A tumors were excised from wild type, IL- $6^{-/-}$, and IL- $10^{-/-}$BALB/c mice, manually teased into small pieces, incubated overnight, and the supernatants analyzed by ELISA for IL-6 and IL-10. Cytokine levels were normalized to $1 \mathrm{~g}$ of tumor tissue per $\mathrm{mL}$ of media. Data are pooled from four independent experiments. Statistical significance was assessed by $t$-test. 


\section{Figure 7}

A
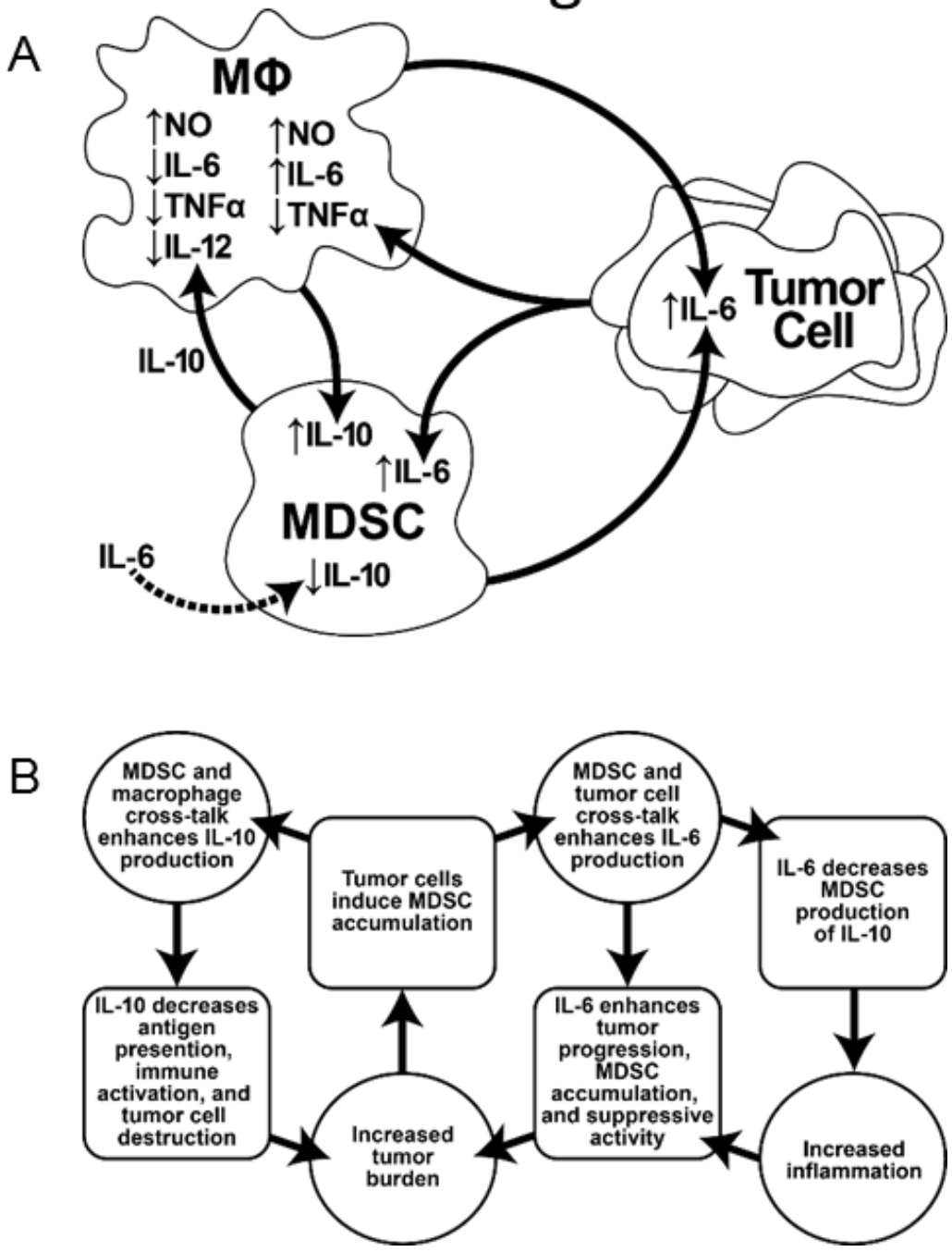

Figure 7: Summary of cross-talk between MDSC, macrophages, and tumor cells. (A) Cross-talk with respect to IL-10, IL-6, IL-12, TNF $\alpha$, and NO. Solid arrows indicate direct effects mediated by the cell type or IL-10. Dashed arrow indicates an indirect effect by IL-6. (B) Potential cycle by IL-6, IL-10, and MDSC cross-talk promotes inflammation and tumor progression. 


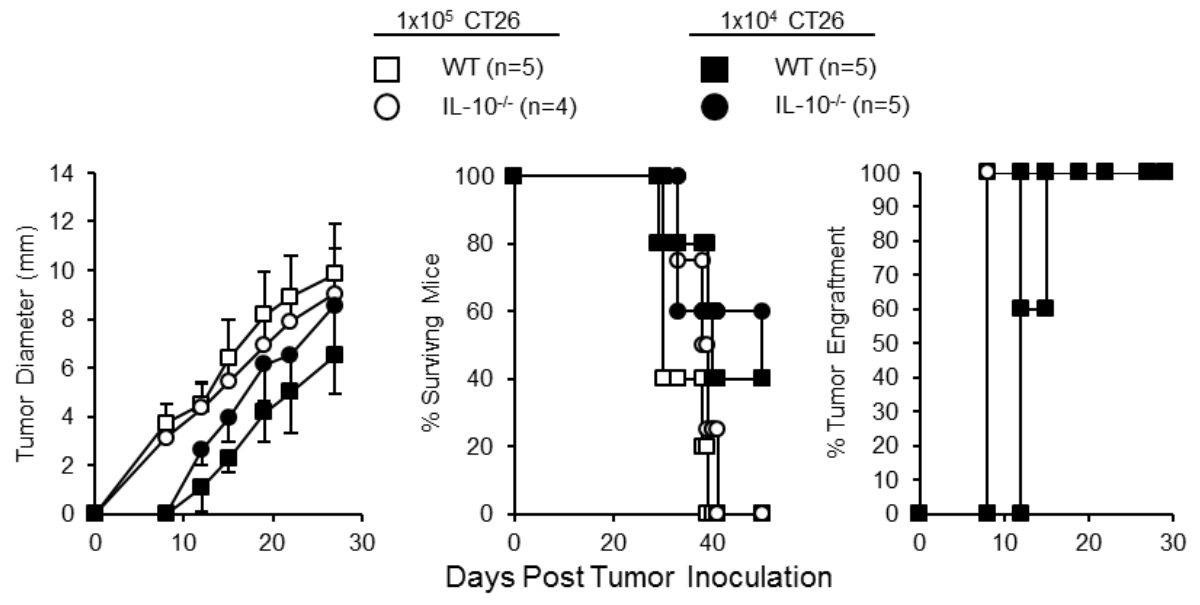

Supplemental Figure 1A: Tumor progression, survival time, and engraftment are similar in $\mathrm{BALB} / \mathrm{c}$ wild type and IL-10/- mice inoculated with $1 \times 10^{5}$ or $1 \times 10^{4} \mathrm{CT} 26$ tumor cells.

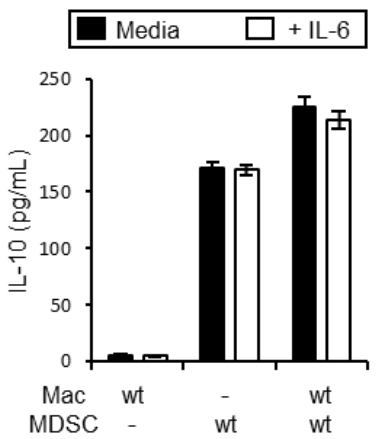

Supplemental Figure 1B: Exogenous IL-6 does not impact MDSC production of IL-10. Macrophages and /or MDSC from wild type or IL-10-/ BALB/c mice with 4T1 tumors were cultured overnight in the presence of $5 \mathrm{ng} / \mathrm{mL}$ recombinant IL-6 and the supernatants were assayed for IL-10. Data are from one of three independent experiments. Statistical significance was assessed by $t$-test.
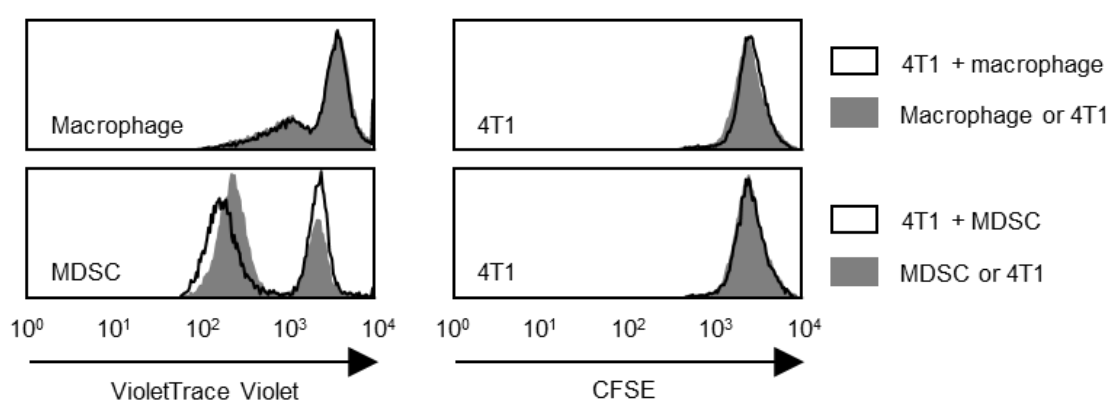

Supplemental Figure 1C: MDSC, but not tumor cells or macrophages, proliferate during coculture. MDSC and macrophages were labeled with VioletTracer and tumor cells were labeled with CFSE. Cells were either cultured alone, or MDSC or macrophages were cultured with 4T1 tumor cells. Cultures were incubated overnight and cell proliferation was assessed the following day by flow cytometry. Data are from one of three independent experiments. 

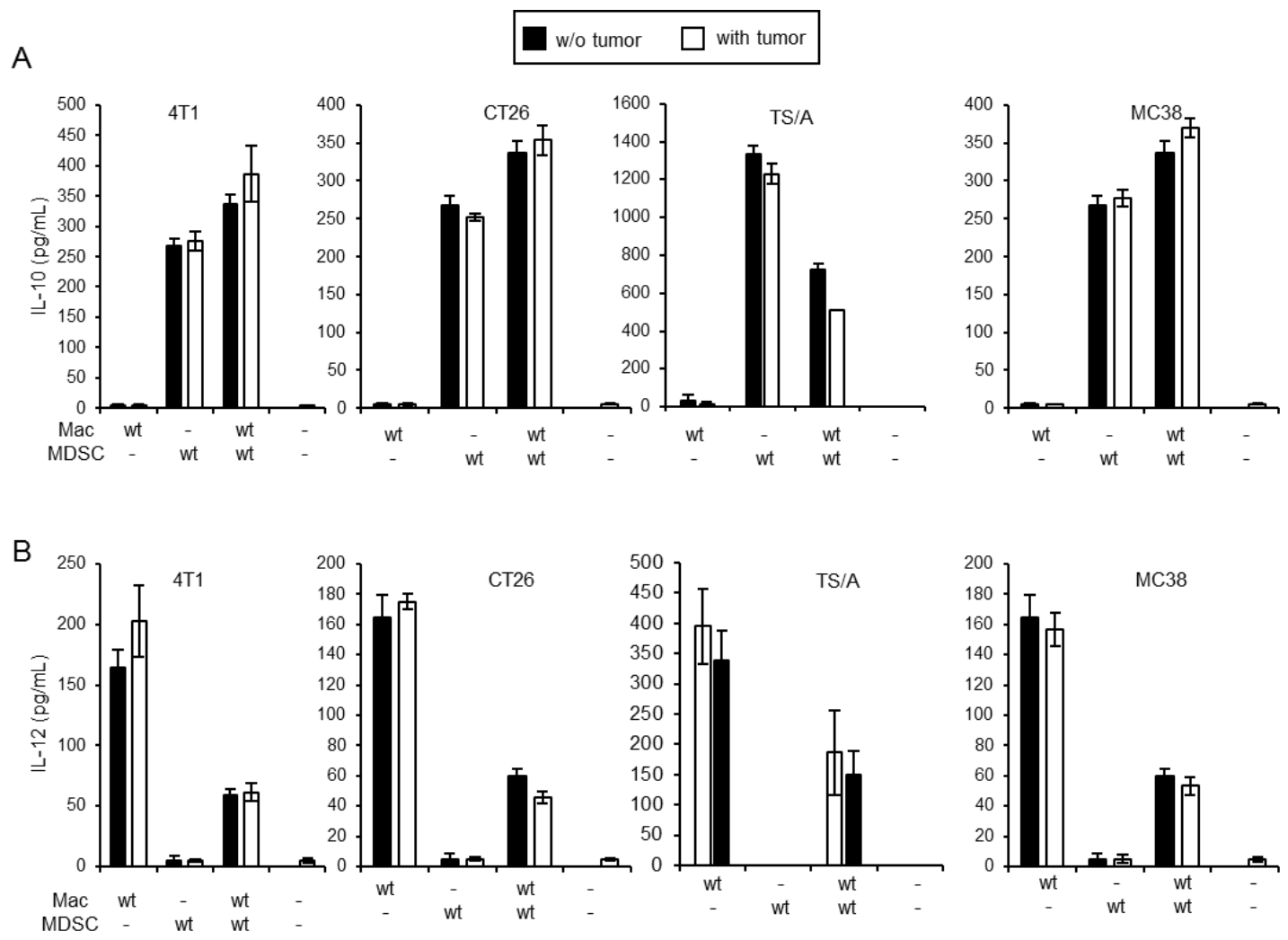

C
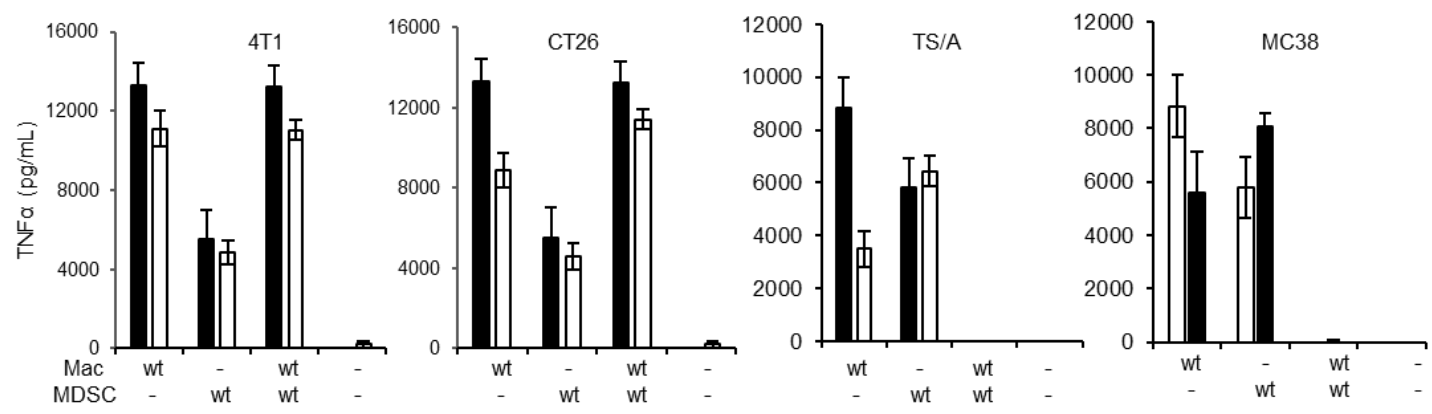

Supplemental Figure 2: Tumor cells do not affect macrophage and/or MDSC production of IL-12 or IL-10, and MDSC do not affect tumor cell and macrophage production of TNFa. 4T1, CT26, TS/A, or MC38 tumor cells were cultured with or without macrophages and/or MDSC from 4T1-tumor-bearing mice. Supernatants were analyzed for (A) IL-10, (B) IL-12, and (C) TNFa. Data are from one of five independent experiments. Statistical significance was assessed by $t$-test. 
Supplemental Table 1: IL-10-deficiency and IL-6-deficiency modestly alter MDSC production of other cytokines and cross-talk with macrophages

\begin{tabular}{|c|c|c|c|c|c|c|c|}
\hline \multirow[t]{2}{*}{ Cells in Culture } & \multicolumn{7}{|c|}{ Cytokine $(\mathrm{pg} / \mathrm{mL})$ or Nitric Oxide $(\mu \mathrm{M})^{\mathrm{a}}$} \\
\hline & TGF- $\beta 1$ & TGF- $\beta 2$ & IL-1 $\beta$ & CCL2 & MIP- $1 \alpha$ & VEGF & NO \\
\hline Macrophages & $386.7 \pm 20.9$ & $122.1 \pm 3.71$ & $18.40 \pm 2.89$ & $2414 \pm 473$ & $9410 \pm 131$ & $8.760 \pm 1.70$ & $10.80 \pm 0.49$ \\
\hline WT MDSC & $294.6 \pm 36.2$ & $72.64 \pm 9.24$ & $88.16 \pm 13.8$ & $35.17 \pm 3.77$ & $6978 \pm 2.79$ & $2.690 \pm 0.17$ & 0 \\
\hline $\mathrm{IL}-10^{-/} \mathrm{MDSC}$ & $369.6 \pm 29.8$ & $83.25 \pm 5.33$ & $86.28 \pm 2.15$ & $47.35 \pm 3.21$ & $6108 \pm 25.0$ & $2.680 \pm 0.01$ & 0 \\
\hline $\mathrm{IL}-6 * \mathrm{MDSC}$ & $475.0 \pm 15.1$ & $125.0 \pm 15.7$ & $128.0 \pm 12.0$ & $59.90 \pm 3.76$ & $6144 \pm 157$ & $6.730 \pm 0.73$ & 0 \\
\hline $\mathrm{Mac}+\mathrm{WT}$ MDSC & $435.9 \pm 7.89^{b}$ & $108.3 \pm 8.51^{\mathrm{b}}$ & $114.3 \pm 14.3^{\mathrm{d}}$ & $2680 \pm 166^{\mathrm{d}}$ & $9804 \pm 117^{b}$ & $26.31 \pm 4.40^{c}$ & $13.34 \pm 0.09^{c}$ \\
\hline $\mathrm{Mac}+\mathrm{IL}-10^{-} \mathrm{MDSC}$ & $501.7 \pm 65.8^{b}$ & $110.9 \pm 9.89^{b}$ & $131.2 \pm 5.31^{\mathrm{d}}$ & $1738 \pm 244^{b}$ & $9215 \pm 24.0^{\mathrm{b}}$ & $22.48 \pm 3.76^{c}$ & $8.140 \pm 0.35^{b}$ \\
\hline $\mathrm{Mac}+\mathrm{IL}-6^{-/} \mathrm{MDSC}$ & $320.7 \pm 26.7^{b}$ & $82.27 \pm 1.94^{b}$ & $98.83 \pm 8.01^{\mathrm{d}}$ & $1777 \pm 100^{\mathrm{b}}$ & $8459 \pm 101^{b}$ & $18.59 \pm 0.81^{c}$ & $12.37 \pm 1.08^{c}$ \\
\hline
\end{tabular}

${ }^{a} 7.5 \times 10^{5}$ wild type BALB/c macrophages and/or MDSC from wild type, IL-10 ${ }^{-/}$, or IL$6^{-1-} \mathrm{BALB} / \mathrm{c}$ mice with $4 \mathrm{~T} 1$ tumors were cultured for $16 \mathrm{hr}$ with $100 \mathrm{ng} / \mathrm{mL}$ LPS and $20 \mathrm{U} / \mathrm{mL}$ IFN $\gamma$, and supernatants were assayed for TGF $\beta 1$, TGF $\beta 2$, TGF $\beta 3$, GM-CSF, IL$1 \beta$, CCL2, MIP-1 $\alpha$, IL-4, IL-13, IL-23, and VEGF. Cytokine values are the average of two measurements of pooled supernatants from three individual cultures \pm standard deviation. Nitric oxide values are averaged from three individual cultures \pm standard deviation. TGF 33 , GM-CSF, IL-4, IL-13, and IL-23 were not detected in supernatants of wild type MDSC and macrophage monocultures or co-cultures.

${ }^{\mathrm{b}}$ level is decreased by co-culture

${ }^{\mathrm{c}}$ level is increased by co-culture

${ }^{\mathrm{d}}$ level is not changed by co-culture 
Supplemental Table 2: NO has pro- and anti-tumor effects.

\begin{tabular}{|c|c|}
\hline Pro-tumor effects of NO/NOS2 & References \\
\hline $\begin{array}{l}\text { - C57BL/6 mice bearing NOS2 } 2^{-/-} \mathrm{C} 6 \text { gliomas have reduced tumor } \\
\text { growth compared to mice with } \mathrm{NOS} 2^{+/+} \text {parental C6 tumors }\end{array}$ & $(40)$ \\
\hline $\begin{array}{l}\text { NOS2 } 2^{-/-} \text {mice treated with urethane developed more lung } \\
\text { carcinomas }\end{array}$ & $(41)$ \\
\hline $\begin{array}{l}\text { - } \mathrm{NOS}^{-/-} \text {mice with B16 melanomas developed larger metastases } \\
\text { than wild type mice }\end{array}$ & $(42)$ \\
\hline $\begin{array}{l}\text { - NOS } 2^{+/+} \text {murine colorectal carcinoma cells are three-fold more } \\
\text { invasive than their NOS } 2^{-/-} \text {counterparts. Treatment of the NOS2- } \\
\text { /- or NOS } 2^{+/+} \text {cells with a NO-donor or NOS } 2 \text { inhibitor increased } \\
\text { and decreased invasiveness, respectively. }\end{array}$ & $(43)$ \\
\hline $\begin{array}{l}\text { - Stimulation of EMT-6 breast carcinoma cells to produce NO } \\
\text { increased tumor growth and metastasis, while inhibition of NO } \\
\text { reversed the effects }\end{array}$ & $(44)$ \\
\hline $\begin{array}{l}\text { - NO produced by monocytic MDSC inhibited T cell activation } \\
\text { and caused immune suppression that promotes tumor growth }\end{array}$ & $(45)$ \\
\hline Anti-tumor effects of NO/NOS2 & References \\
\hline $\begin{array}{l}\text { - Transgenic NOS2 }{ }^{-/-} \mathrm{APC}^{\mathrm{Min}} \text { mice develop more spontaneous } \\
\text { intestinal adenomas }\end{array}$ & $(13,46)$ \\
\hline $\begin{array}{l}\text { - } \mathrm{NOS}^{-/-} \mathrm{Py}-\mathrm{MT} \text { and } \mathrm{APC} \\
\text { compared to } \mathrm{NOS} 2^{+/+} \text {mice. }\end{array}$ & $(47,48)$ \\
\hline $\begin{array}{l}\text { - NOS } 2^{-/-} \text {mice with transplanted or chemically induced tumors } \\
\text { have increased vascularization. }\end{array}$ & $(41,49)$ \\
\hline $\begin{array}{l}\text { - Host-derived NO inhibits the growth of methlcholanthrene- } \\
\text { induced fibrosarcomas. }\end{array}$ & $(50)$ \\
\hline $\begin{array}{l}\text { - NO is cytotoxic for tumor cells when produced by M1 } \\
\text { macrophages. }\end{array}$ & $(51,52)$ \\
\hline
\end{tabular}




\section{References}

1. Shiao, S. L., A. P. Ganesan, H. S. Rugo, and L. M. Coussens. 2011. Immune microenvironments in solid tumors: new targets for therapy. Genes Dev 25: 25592572.

2. Yang, H., and K. J. Tracey. 2010. Targeting HMGB1 in inflammation. Biochim Biophys Acta 1799: 149-156.

3. Gabrilovich, D. I., S. Ostrand-Rosenberg, and V. Bronte. 2012. Coordinated regulation of myeloid cells by tumours. Nat Rev Immunol 12: 253-268.

4. Viola, A., A. Sarukhan, V. Bronte, and B. Molon. 2012. The pros and cons of chemokines in tumor immunology. Trends Immunol 33: 496-504.

5. Sica, A., and A. Mantovani. 2012. Macrophage plasticity and polarization: in vivo veritas. J Clin Invest 122: 787-795.

6. Ostrand-Rosenberg, S., and P. Sinha. 2009. Myeloid-derived suppressor cells: linking inflammation and cancer. J Immunol 182: 4499-4506.

7. Bunt, S. K., V. K. Clements, E. M. Hanson, P. Sinha, and S. Ostrand-Rosenberg. 2009. Inflammation enhances myeloid-derived suppressor cell cross-talk by signaling through Toll-like receptor 4. J Leukoc Biol 85: 996-1004.

8. Sinha, P., V. K. Clements, S. K. Bunt, S. M. Albelda, and S. Ostrand-Rosenberg. 2007. Cross-talk between myeloid-derived suppressor cells and macrophages subverts tumor immunity toward a type 2 response. J Immunol 179: 977-983. 
9. Becker, C., M. C. Fantini, S. Wirtz, A. Nikolaev, H. A. Lehr, P. R. Galle, S. RoseJohn, and M. F. Neurath. 2005. IL-6 signaling promotes tumor growth in colorectal cancer. Cell Cycle 4: 217-220.

10. Su, Y. W., T. X. Xie, D. Sano, and J. N. Myers. 2011. IL-6 stabilizes Twist and enhances tumor cell motility in head and neck cancer cells through activation of casein kinase 2. PLoS One 6: e19412.

11. Santer, F. R., K. Malinowska, Z. Culig, and I. T. Cavarretta. 2010. Interleukin-6 trans-signalling differentially regulates proliferation, migration, adhesion and maspin expression in human prostate cancer cells. Endocr Relat Cancer 17: 241253.

12. Balkwill, F. 2006. TNF-alpha in promotion and progression of cancer. Cancer Metastasis Rev 25: 409-416.

13. Hussain, S. P., G. E. Trivers, L. J. Hofseth, P. He, I. Shaikh, L. E. Mechanic, S. Doja, W. Jiang, J. Subleski, L. Shorts, D. Haines, V. E. Laubach, R. H. Wiltrout, D. Djurickovic, and C. C. Harris. 2004. Nitric oxide, a mediator of inflammation, suppresses tumorigenesis. Cancer Res 64: 6849-6853.

14. Bronte, V., and P. Zanovello. 2005. Regulation of immune responses by Larginine metabolism. Nat Rev Immunol 5: 641-654.

15. Mocellin, S., F. M. Marincola, and H. A. Young. 2005. Interleukin-10 and the immune response against cancer: a counterpoint. J Leukoc Biol 78: 1043-1051.

16. Sinha, P., K. H. Parker, L. Horn, and S. Ostrand-Rosenberg. 2012. Tumorinduced myeloid-derived suppressor cell function is independent of IFN-gamma and IL-4Ralpha. Eur J Immunol 42: 2052-2059. 
17. Bunt, S. K., P. Sinha, V. K. Clements, J. Leips, and S. Ostrand-Rosenberg. 2006. Inflammation induces myeloid-derived suppressor cells that facilitate tumor progression. J Immunol 176: 284-290.

18. Sinha, P., V. K. Clements, and S. Ostrand-Rosenberg. 2005. Reduction of myeloid-derived suppressor cells and induction of M1 macrophages facilitate the rejection of established metastatic disease. J Immunol 174: 636-645.

19. Lippitz, B. E. 2013. Cytokine patterns in patients with cancer: a systematic review. Lancet Oncol 14: e218-228.

20. Hirotake Tsukamoto, R. N., Satoru Senju, and Yasuharu Nishimura. 2013. Myeloid-Derived Suppressor Cells Attenuate TH1 Development through IL-6 Production to Promote Tumor Progression. Cancer Immunol Res: 13.

21. Berg, D. J., N. Davidson, R. Kuhn, W. Muller, S. Menon, G. Holland, L. Thompson-Snipes, M. W. Leach, and D. Rennick. 1996. Enterocolitis and colon cancer in interleukin-10-deficient mice are associated with aberrant cytokine production and CD4(+) TH1-like responses. J Clin Invest 98: 1010-1020.

22. Neven, B., E. Mamessier, J. Bruneau, S. Kaltenbach, D. Kotlarz, F. Suarez, J. Masliah-Planchon, K. Billot, D. Canioni, P. Frange, I. Radford-Weiss, V. Asnafi, D. Murugan, C. Bole, P. Nitschke, O. Goulet, J. L. Casanova, S. Blanche, C. Picard, O. Hermine, F. Rieux-Laucat, N. Brousse, F. Davi, V. Baud, C. Klein, B. Nadel, F. Ruemmele, and A. Fischer. 2013. A Mendelian predisposition to B-cell lymphoma caused by IL-10R deficiency. Blood 122: 3713-3722.

23. Emmerich, J., J. B. Mumm, I. H. Chan, D. LaFace, H. Truong, T. McClanahan, D. M. Gorman, and M. Oft. 2012. IL-10 directly activates and expands tumor- 
resident CD8(+) T cells without de novo infiltration from secondary lymphoid organs. Cancer Res 72: 3570-3581.

24. Mumm, J. B., J. Emmerich, X. Zhang, I. Chan, L. Wu, S. Mauze, S. Blaisdell, B. Basham, J. Dai, J. Grein, C. Sheppard, K. Hong, C. Cutler, S. Turner, D. LaFace, M. Kleinschek, M. Judo, G. Ayanoglu, J. Langowski, D. Gu, B. Paporello, E. Murphy, V. Sriram, S. Naravula, B. Desai, S. Medicherla, W. Seghezzi, T. McClanahan, S. Cannon-Carlson, A. M. Beebe, and M. Oft. 2011. IL-10 elicits IFNgamma-dependent tumor immune surveillance. Cancer Cell 20: 781-796.

25. Sato, T., M. Terai, Y. Tamura, V. Alexeev, M. J. Mastrangelo, and S. R. Selvan. 2011. Interleukin 10 in the tumor microenvironment: a target for anticancer immunotherapy. Immunol Res 51: 170-182.

26. Jacobs, F., D. Chaussabel, C. Truyens, V. Leclerq, Y. Carlier, M. Goldman, and B. Vray. 1998. IL-10 up-regulates nitric oxide (NO) synthesis by lipopolysaccharide (LPS)-activated macrophages: improved control of Trypanosoma cruzi infection. Clin Exp Immunol 113: 59-64.

27. Qasimi, P., A. Ming-Lum, A. Ghanipour, C. J. Ong, M. E. Cox, J. Ihle, N. Cacalano, A. Yoshimura, and A. L. Mui. 2006. Divergent mechanisms utilized by SOCS3 to mediate interleukin-10 inhibition of tumor necrosis factor alpha and nitric oxide production by macrophages. J Biol Chem 281: 6316-6324.

28. Smith, C., M. Y. Chang, K. H. Parker, D. W. Beury, J. B. DuHadaway, H. E. Flick, J. Boulden, E. Sutanto-Ward, A. P. Soler, L. D. Laury-Kleintop, L. Mandik-Nayak, R. Metz, S. Ostrand-Rosenberg, G. C. Prendergast, and A. J. 
Muller. 2012. IDO is a nodal pathogenic driver of lung cancer and metastasis development. Cancer Discov 2: 722-735.

29. Marigo, I., E. Bosio, S. Solito, C. Mesa, A. Fernandez, L. Dolcetti, S. Ugel, N. Sonda, S. Bicciato, E. Falisi, F. Calabrese, G. Basso, P. Zanovello, E. Cozzi, S. Mandruzzato, and V. Bronte. 2010. Tumor-induced tolerance and immune suppression depend on the C/EBPbeta transcription factor. Immunity 32: 790-802.

30. Bunt, S. K., L. Yang, P. Sinha, V. K. Clements, J. Leips, and S. OstrandRosenberg. 2007. Reduced inflammation in the tumor microenvironment delays the accumulation of myeloid-derived suppressor cells and limits tumor progression. Cancer Res 67: 10019-10026.

31. Ouyang, W., S. Rutz, N. K. Crellin, P. A. Valdez, and S. G. Hymowitz. 2011. Regulation and functions of the IL-10 family of cytokines in inflammation and disease. Annu Rev Immunol 29: 71-109.

32. Lee, J. H., G. T. Lee, S. H. Woo, Y. S. Ha, S. J. Kwon, W. J. Kim, and I. Y. Kim. 2013. BMP-6 in renal cell carcinoma promotes tumor proliferation through IL-10dependent M2 polarization of tumor-associated macrophages. Cancer Res 73: 3604-3614.

33. Dennis, K. L., Y. Wang, N. R. Blatner, S. Wang, A. Saadalla, E. Trudeau, A. Roers, C. T. Weaver, J. J. Lee, J. A. Gilbert, E. B. Chang, and K. Khazaie. 2013. Adenomatous polyps are driven by microbe-instigated focal inflammation and are controlled by IL-10-producing T cells. Cancer Res 73: 5905-5913. 
34. Tanikawa, T., C. M. Wilke, I. Kryczek, G. Y. Chen, J. Kao, G. Nunez, and W. Zou. 2012. Interleukin-10 ablation promotes tumor development, growth, and metastasis. Cancer Res 72: 420-429.

35. Niemand, C., A. Nimmesgern, S. Haan, P. Fischer, F. Schaper, R. Rossaint, P. C. Heinrich, and G. Muller-Newen. 2003. Activation of STAT3 by IL-6 and IL-10 in primary human macrophages is differentially modulated by suppressor of cytokine signaling 3. Journal of immunology 170: 3263-3272.

36. Vallania, F., D. Schiavone, S. Dewilde, E. Pupo, S. Garbay, R. Calogero, M. Pontoglio, P. Provero, and V. Poli. 2009. Genome-wide discovery of functional transcription factor binding sites by comparative genomics: the case of Stat3. Proceedings of the National Academy of Sciences of the United States of America 106: $5117-5122$.

37. Hutchins, A. P., D. Diez, and D. Miranda-Saavedra. 2013. The IL-10/STAT3mediated anti-inflammatory response: recent developments and future challenges. Brief Funct Genomics 12: 489-498.

38. Morales, J. K., S. J. Saleem, R. K. Martin, B. L. Saunders, B. O. Barnstein, T. W. Faber, N. A. Pullen, E. M. Kolawole, K. B. Brooks, S. K. Norton, J. Sturgill, L. Graham, H. D. Bear, J. F. Urban, Jr., C. S. Lantz, D. H. Conrad, and J. J. Ryan. 2014. Myeloid-derived suppressor cells enhance IgE-mediated mast cell responses. J Leukoc Biol 95: 643-650.

39. Saleem, S. J., R. K. Martin, J. K. Morales, J. L. Sturgill, D. R. Gibb, L. Graham, H. D. Bear, M. H. Manjili, J. J. Ryan, and D. H. Conrad. 2012. Cutting edge: mast 
cells critically augment myeloid-derived suppressor cell activity. J Immunol 189: 511-515.

40. Yamaguchi, S., H. S. Bell, J. Shinoda, M. C. Holmes, S. B. Wharton, and I. R. Whittle. 2002. Glioma tumourgenicity is decreased by iNOS knockout: experimental studies using the C6 striatal implantation glioma model. Br J Neurosurg 16: 567-572.

41. Kisley, L. R., B. S. Barrett, A. K. Bauer, L. D. Dwyer-Nield, B. Barthel, A. M. Meyer, D. C. Thompson, and A. M. Malkinson. 2002. Genetic ablation of inducible nitric oxide synthase decreases mouse lung tumorigenesis. Cancer Res 62: 6850-6856.

42. Shi, Q., Q. Xiong, B. Wang, X. Le, N. A. Khan, and K. Xie. 2000. Influence of nitric oxide synthase II gene disruption on tumor growth and metastasis. Cancer research 60: 2579-2583.

43. Siegert, A., C. Rosenberg, W. D. Schmitt, C. Denkert, and S. Hauptmann. 2002. Nitric oxide of human colorectal adenocarcinoma cell lines promotes tumour cell invasion. Brit J Cancer 86: 1310-1315.

44. Edwards, P., J. C. Cendan, D. B. Topping, L. L. Moldawer, S. MacKay, E. Copeland, and D. S. Lind. 1996. Tumor cell nitric oxide inhibits cell growth in vitro, but stimulates tumorigenesis and experimental lung metastasis in vivo. The Journal of surgical research 63: 49-52.

45. Youn, J. I., S. Nagaraj, M. Collazo, and D. I. Gabrilovich. 2008. Subsets of myeloid-derived suppressor cells in tumor-bearing mice. J Immunol 181: 57915802. 
46. Scott, D. J., M. A. Hull, E. J. Cartwright, W. K. Lam, A. Tisbury, R. Poulsom, A. F. Markham, C. Bonifer, and P. L. Coletta. 2001. Lack of inducible nitric oxide synthase promotes intestinal tumorigenesis in the $\operatorname{Apc}(\mathrm{Min} /+)$ mouse. Gastroenterology 121: 889-899.

47. Ellies, L. G., M. Fishman, J. Hardison, J. Kleeman, J. E. Maglione, C. K. Manner, R. D. Cardiff, and C. L. MacLeod. 2003. Mammary tumor latency is increased in mice lacking the inducible nitric oxide synthase. Int J Cancer 106: 1-7.

48. Ahn, B., and H. Ohshima. 2001. Suppression of intestinal polyposis in Apc(Min/+) mice by inhibiting nitric oxide production. Cancer Res 61: 83578360.

49. Konopka, T. E., J. E. Barker, T. L. Bamford, E. Guida, R. L. Anderson, and A. G. Stewart. 2001. Nitric oxide synthase II gene disruption: implications for tumor growth and vascular endothelial growth factor production. Cancer Res 61: 31823187.

50. Wei, D., E. L. Richardson, K. Zhu, L. Wang, X. Le, Y. He, S. Huang, and K. Xie. 2003. Direct demonstration of negative regulation of tumor growth and metastasis by host-inducible nitric oxide synthase. Cancer Res 63: 3855-3859.

51. Nathan, C. F., and J. B. Hibbs, Jr. 1991. Role of nitric oxide synthesis in macrophage antimicrobial activity. Curr Opin Immunol 3: 65-70.

52. Farias-Eisner, R., M. P. Sherman, E. Aeberhard, and G. Chaudhuri. 1994. Nitric oxide is an important mediator for tumoricidal activity in vivo. Proc Natl Acad Sci U S A 91: 9407-9411. 


\title{
Chapter 3: Myeloid-derived suppressor cell survival and function are regulated by the transcription factor $\mathrm{Nrf} 2$
}

\begin{abstract}
Tumor-induced myeloid-derived suppressor cells (MDSC) contribute to immune suppression in tumor-bearing individuals and are a major obstacle to effective immunotherapy. Reactive oxygen species (ROS) are one of the mechanisms used by MDSC to suppress T cell activation. Although ROS are toxic to most cells, MDSC survive despite their elevated content and release of ROS. Nuclear factor erythroid derived 2-like $2(\mathrm{Nrf} 2)$ is a transcription factor that regulates a battery of genes which attenuates oxidative stress. Therefore, we hypothesized that MDSC resistance to ROS may be due to their up-regulation of Nrf2. To test this hypothesis, we utilized BALB/c and C57BL/6 mice bearing 4T1 mammary carcinoma and MC38 colon carcinoma, respectively. Nrf2 enhanced MDSC suppressive activity by increasing MDSC production of $\mathrm{H}_{2} \mathrm{O}_{2}$, and increased the quantity of tumor-infiltrating MDSC by reducing their oxidative stress and rate of apoptosis. Nrf2 did not affect circulating levels of MDSC in tumor-bearing mice since the decreased apoptotic rate of tumor-infiltrating MDSC was balanced by a decreased rate of differentiation from bone marrow progenitor cells. These results demonstrate that $\mathrm{Nrf} 2$ regulates the generation, survival and suppressive potency of MDSC, and that a feedback homeostatic mechanism maintains a steady-state level of circulating MDSC in tumor-bearing individuals.
\end{abstract}




\section{Introduction}

The microenvironment of solid tumors (tumor microenvironment; TME) is frequently inflamed and oxidatively stressed due to hypoxia, the presence of reactive oxygen species (ROS), and multiple pro-inflammatory mediators (1). Therefore, cells in this environment must mitigate oxidative radicals in order to survive. Tumor cells survive this environment by having elevated levels of stabilized nuclear factor (erythroid-2)related factor 2 (Nrf2), which enhances tumor cell proliferation and resistance to chemotherapy, and promotes tumor growth (2-4). Nrf2 is a basic-leucine-zipper (bZIP) transcription factor that is ubiquitously expressed in many tissues and cells $(2,5-7)$. Under normal redox conditions, Nrf2 is restricted to the cytoplasm by Kelch-like ECHassociated protein 1 (Keap1), which promotes the polyubiquitination of Nrf2, leading to its destruction by the 26s proteasome (8-12). Oxidative stress stabilizes Nrf2 by oxidizing key thiol residues on Keap1, which causes conformational changes of Keap1 that prevent Nrf2 polyubiquitination and subsequent degradation (13-15). Nrf2 can also be stabilized through direct phosphorylation by kinases involved in inflammatory signaling cascades (e.g. KRAS, MYC, PKC, ERK, MAPK, and p38) (16-21). Once stabilized, Nrf2 translocates to the nucleus where it heterodimerizes with other bZIP transcription factors including Jun (c-Jun, Jun-D, and Jun-B) and small Maf (MafG, MafK, and MafF) (22-27) and up-regulates genes containing an antioxidant response element (ARE) in their promoter (28-30). Activation of these antioxidant genes quenches oxidative stress and promotes detoxification, thereby protecting cells from oxidative toxicity.

Cells of the immune system are also present in the TME and must protect 
themselves against oxidative radicals. Myeloid-derived suppressor cells (MDSC) are immature myeloid cells that suppress T cell activation and proliferation (31), perturb naïve $\mathrm{T}$ cell trafficking to lymph nodes (32), impair NK cell cytotoxicity (33), induce T regulatory cells (34), and skew macrophages to a tumor promoting (type II) phenotype (35). MDSC are present in most solid tumors where they contribute to oxidative stress by their production of superoxide (36). Superoxide produced by MDSC rapidly reacts with a large number of molecules to form ROS such as hydrogen peroxide $\left(\mathrm{H}_{2} \mathrm{O}_{2}\right)$, hydroxyl radical, hypochlorous acid, and peroxynitrate (ONOO-; e.g. PNT), which damage proteins, lipids, and nucleic acids, enhance inflammation, and promote apoptosis. $\mathrm{H}_{2} \mathrm{O}_{2}$ reduces $\mathrm{T}$ cell expression of $\mathrm{CD} 3 \zeta$ chain thereby limiting their ability to become activated and mediate anti-tumor immunity $(37,38)$. Nitration/nitrosylation of T cell receptors (39) and MHC class I molecules (40) by PNT disrupts T cell-tumor antigen interactions and renders tumor cells resistant to CTL-mediated lysis. Despite their high intracellular content of ROS and their secretion of ROS, which constantly exposes them to oxidative stress, MDSC accumulate and function in tumor-bearing patients and animals. Given that tumor cells are protected from oxidative stress by Nrf2, we hypothesized that Nrf2 may also protect MDSC from oxidative stress.

To elucidate the role of Nrf2 in MDSC, we examined the survival time of tumorbearing BALB/c and C57BL/6 $\mathrm{Nrf}^{+/+}$and $\mathrm{Nrf2}^{-/-}$mice, and the generation, survival, suppressive potency, and tumor-infiltration of MDSC in these mice. Wild type tumorbearing mice have a decreased survival time and have more tumor-infiltrating and more suppressive MDSC compared to Nrf2-deficient mice. The increase in tumor-infiltrating MDSC is the result of a reduced rate of MDSC apoptosis. However, Nrf2 does not affect 
the level of MDSC in the periphery because a homeostatic regulatory mechanism increases MDSC generation from bone marrow progenitor cells.

\section{Materials and Methods}

Mice

BALB/c $\left(\mathrm{Nrf}^{+/+}\right), \mathrm{BALB} / \mathrm{c} \mathrm{Nrf2} 2^{-/}, \mathrm{C} 57 \mathrm{BL} / 6\left(\mathrm{Nrf}^{+/+}\right)$, and C57BL/6 Nrf2 ${ }^{-/-}$mice were bred in the UMBC animal facility from stock obtained from The Jackson Laboratory (C57BL/6 and BALB/c) or provided by Dr. Masayuki Yamamoto (RIKEN, Japan; BALB/c Nrf2 ${ }^{+-}$) and Dr. Shyam Biswal (Johns Hopkins School of Public Health; $\left.\mathrm{C} 57 \mathrm{BL} / 6 \mathrm{Nrf2}{ }^{---}\right)$. BALB/c Nrf2 $2^{-{ }^{-}}$mice were generated by mating BALB/c Nrf2 ${ }^{+/-} \mathrm{x}$ $\mathrm{BALB} / \mathrm{c} \mathrm{Nrf2}{ }^{+/-}$mice. BALB/c Nrf2 $2^{-/}$and $\mathrm{Nrf2}^{+/+}$offspring were identified by PCR typing (Supplemental Figure 1A, 1B). DNA was isolated from pups (Qiagen, QIAamp DNA blood mini kit, per the manufacturer's protocol), and amplified using primers specific to $\mathrm{Nrf} 2$ and lac $\mathrm{Z}$ under the following conditions: $94^{\circ} \mathrm{C}$ melting for 30 seconds, $56^{\circ} \mathrm{C}$ annealing for 30 seconds, $72^{\circ} \mathrm{C}$ extension for one minute, for 30 cycles.

Homozygous knockouts have a 400kb band; heterozygotes 400 and 734kb bands; and homozygous wild type mice a $734 \mathrm{~kb}$ band. BALB/c Nrf2 ${ }^{+/+}$littermates from these matings served as controls. C57BL/6 $\mathrm{Nrf}^{-/-}$mice were generated by crossing $\mathrm{C} 57 \mathrm{BL} / 6$ $\mathrm{Nrf2}^{-/-}$x C57BL/6 Nrf2 ${ }^{-/-}$mice. Nrf2-deficiency was further verified by qPCR analysis glutamate-cysteine ligase, modifier subunit (GCLM), heme oxygenase 1 (HO-1), catalase, and $\mathrm{NAD}(\mathrm{P}) \mathrm{H}$ dehydrogenase, quinone 1(NQO1), genes that are regulated by Nrf2 (Supplemental Figure 1B, 1C). $10^{7}$ MDSC from 4T1-bearing Nrf2 ${ }^{+/+}$or Nrf2 ${ }^{-/}$ 
mice were suspended in IMDM supplemented with 10\% Fetal Clone I (Thermo Scientific, Waltham, MA), $1 \%$ penicillin-streptomycin, $1 \%$ glutamax, and $0.1 \%$ gentamycin, and plated in $35 \mathrm{~mm}$ petri dishes in the presence of tert-butylhydroquinone (tbHQ, $50 \mu \mathrm{M}$; Sigma Aldrich, St. Louis, MO) or vehicle control (DMSO). Cultures were incubated for 6 hours $\left(37^{\circ} \mathrm{C}, 5 \% \mathrm{CO}_{2}\right)$ and harvested. RNA was isolated with TRIzol reagent (ThermoFisher, Grand Island, NY) and chloroform extraction. cDNA was synthesized using a Maxima First Strand cDNA Synthesis Kit (ThermoFisher) as per the manufacture's protocol. Quantitative PCR was performed using KiCqStart SYBR Green qPCR ReadyMix (Sigma Aldrich) with $100 \mathrm{nM}$ of forward and reverse primers (Supplemental Figure 1B) on a CFX96 Real-Time System (Bio-Rad, Hercules, CA) under the following conditions: $95^{\circ} \mathrm{C}$ melting for 10 seconds, $57^{\circ} \mathrm{C}$ annealing/extension for 30 seconds, for 40 cycles. Data were analyzed using CFX Manager (Bio-Rad), and Nrf2-regulated genes were normalized to the housekeeping gene L32 by the $\Delta \mathrm{C}_{\mathrm{T}}$ method.

\section{Tumor cells and tumor growth}

BALB/c-derived 4T1 mammary carcinoma and C57BL/6-derived MC38 colon carcinoma were maintained as described (41), and have been in the authors' lab for more than 15 and 8 years, respectively. 4T1 cells were originally obtained from Dr. Fred Miller (Karmanos Cancer Center) and MC38 cells from Dr. Dmitry Gabrilovich (Wistar

Institute). Cell lines were routinely checked for mycoplasma and early freeze-downs were preferentially used. Mice were inoculated in the abdominal mammary gland with $100 \mu \mathrm{L}$ DMEM containing $7 \times 10^{3}$ or $10^{5} 4 \mathrm{~T} 1$ cells, or in the flank with $5 \times 10^{5}$ MC38 cells. Primary tumors were measured as described (42). Survival time was recorded when mice 
became moribund and were euthanized. All animal procedures were approved by the UMBC Institutional Animal Care and Use Committee.

In vivo generation of MDSC

MDSC were harvested from the peripheral blood of tumor-bearing mice as described (41). Briefly, 4T1 tumor-bearing Nrf2 ${ }^{+/+}$and $\mathrm{Nrf2}^{-/-}$mice with tumors $>10 \mathrm{~mm}$ in diameter were bled by submandibular venipuncture into $500 \mu \mathrm{L}$ of a $0.008 \%$ heparin solution. Red blood cells were removed by lysis, and the remaining cells were analyzed by flow cytometry for MDSC. Cell populations containing $>90 \% \mathrm{Gr} 1^{+} \mathrm{CD} 11 \mathrm{~b}^{+}$cells were utilized in all functional assays.

\section{Flow cytometry reagents and antibodies}

Monoclonal antibodies rat anti-mouse CCR2-PE, CD3-FITC, CD4-APC-Cy7, CD8-APC, CD11b-APC, CD11b-APC-Cy7, CD11c-FITC, CD45-PB, CD45R-PE, CD62L-PE, CXCR4-PE, Ly6C-PE, Gr1-APC-Cy7, Ly6G-Alexa 647, isotype rat IgG2bPE, dichlorofluorescein diacetate (DCFDA), propidium iodide (PI), Annexin V, and 7AAD were from BD Pharmingen (San Diego, CA) or BioLegend (San Diego, CA). Cells were stained with antibodies, with Annexin V and PI, or with DCFDA as described $(43,44)$. Samples were analyzed on a Beckman/Coulter Cyan ADP flow cytometer using Summit software.

\section{Tumor infiltrating cells}

Tumors were dissociated using a modified protocol from the Tissue Dissociation 
Kit (protocol 2.2.1; Miltenyi Biotech, Bergisch Gladbach, Germany) with a GentleMACS Dissociator. Tumors 8 to $12 \mathrm{~mm}$ in diameter were resected from 4T1-bearing mice, cut in half, and each half placed into a GentleMACS C tube containing $5 \mathrm{~mL}$ of dissociation medium (DMEM with 300U/mL collagenase IV, $0.1 \%$ hyaluronidase, and $2 \mathrm{kU} / \mathrm{mL}$

DNase I). Tumors were then minced with scissors into $2-4 \mathrm{~mm}$ pieces, processed on the GentleMACS Dissociator with the program m_impTumor_02, and then rotated (10 rpm; Glas-Col Rotator) at $37^{\circ} \mathrm{C}$ for 40 minutes. Samples were then processed twice on the GentleMACS Dissociator using the program m_impTumor_03. The resulting material was filtered through a $70 \mu \mathrm{M}$ mesh filter and the cells that passed through the filter were washed twice with $10 \mathrm{~mL}$ DMEM (Beckman Allegra 6R centrifuge, 500g for 3 minutes), resuspended in 4mL DMEM, and subjected to ficoll-plaque density gradient centrifugation (Beckman Allegra 6R centrifuge, $1400 \mathrm{~g}$ for $20 \mathrm{~min}$ at $20^{\circ} \mathrm{C}$ ). Live cells were isolated from the ficoll-aqueous interface, washed twice with DMEM, stained with 7AAD and for F4/80, Gr1 or Ly6G and Ly6C, CD3, CD4, CD8, CD11b, CD11c, CD45, and CD45R (B220), and assessed by flow cytometry. Cell percentages were calculated as a percentage of $7 \mathrm{AAD}^{-} \mathrm{CD} 45^{+}$cells.

\section{MDSC differentiation from bone marrow}

MDSCs were generated from bone marrow progenitors as described (45). Briefly, bone marrow was flushed aseptically from the femurs of naïve mice. RBCs were lysed with Gey's solution and the resulting cells were assayed for the percentage of $\mathrm{Gr}^{+} \mathrm{CD} 11 \mathrm{~b}^{+}$cells, and cultured for four days $\left(37^{\circ} \mathrm{C}, 5 \% \mathrm{CO}_{2}\right)$ at $4.2 \times 10^{5}$ cells $/ 2 \mathrm{~mL}$ RPMI medium supplemented with 10\% FCS, $80 \mathrm{ng} / \mathrm{mL}$ IL-6, and $80 \mathrm{ng} / \mathrm{mL}$ GM-CSF/2 
$\mathrm{ml} /$ well in 6 well plates. At the end of culture, the total number of cells was determined, and the percentage of granulocytic $\left(\mathrm{Ly} 6 \mathrm{G}^{\mathrm{hi}} \mathrm{Ly} 6 \mathrm{C}^{\mathrm{lo}} \mathrm{CD} 11 \mathrm{~b}^{+}\right.$; PMN-MDSC) and monocytic $\left(\right.$ Ly6G $^{\text {lo }}$ Ly6C ${ }^{\text {hi }}$ CD11b ; M-MDSC) MDSC was determined by flow cytometry. Number of MDSC $=[($ total number of cells $) \times(\%$ M-MDSC and/or PMN-MDSC $)]$. Ratio Nrf2 $2^{-/-}$ to $\mathrm{Nrf}^{+/+} \mathrm{MDSC}=\left(\right.$ Total Nrf2 ${ }^{-/-}$MDSC $) /\left(\right.$Total Nrf2 $2^{+/+}$MDSC $)$. Ratio PMN-MDSC to M-MDSC $=($ Total PMN-MDSC $) /($ Total M-MDSC $)$

\section{Apoptosis Assay}

Live MDSC were identified as $7 \mathrm{AAD}^{-}$or $\mathrm{PI}^{-}$and $\mathrm{Gr}^{+} \mathrm{CD}^{1} 1 \mathrm{~b}^{+}$or $\mathrm{CD} 11 \mathrm{~b}^{+} \mathrm{Ly}_{6 \mathrm{G}}^{+}$, or $\mathrm{CD} 11 \mathrm{~b}^{+}$Ly6C $\mathrm{C}^{+}$cells. The percent decrease in apoptosis $=100 \mathrm{x}\left[1-\left(\%\right.\right.$ Annexin $\mathrm{V}^{+}$ $\mathrm{Nrf}^{+/+} \mathrm{MDSC} / \%$ Annexin $\left.\left.\mathrm{V}^{+} \mathrm{Nrf2}^{-/-} \mathrm{MDSC}\right)\right]$. For some experiments, MDSC were harvested from the blood of 4T1-tumor bearing mice, re-suspended in HL-1 media (supplemented with $1 \%$ penicillin-streptomycin, $1 \%$ glutamax, and $0.1 \%$ gentamycin), and plated in $6 \mathrm{~cm}$ petri dishes. $\mathrm{Gr} 1^{+} \mathrm{CD} 11 \mathrm{~b}^{+}$cells were assessed for viability by PI staining.

\section{ROS detection}

Reactive oxygen species (ROS) were measured by $\mathrm{H}_{2} \mathrm{O}_{2}$ detection using an Amplex Red Hydrogen Peroxide Assay Kit (Invitrogen) as described (46). Briefly, MDSC were suspended in Dulbecco's PBS at $2.5 \times 10^{6} / \mathrm{mL}$ and $5 \times 10^{4}$ cells $/ 50 \mu 1$ were plated per well in 96 well black, flat-bottom plates (Greiner Bio-One, Monroe, NC). Thirty ng/ml PMA and $50 \mu 1$ Amplex Red Reagent were added to each well. Plates were incubated at $37^{\circ} \mathrm{C}$ and fluorescence (excitation at $530 \mathrm{~nm}$, emission at $590 \mathrm{~nm}$ ) was 
measured for one hour at 5-minute intervals using a Biotek Synergy 2 microplate plate reader (Winooski, VT, USA). A standard curve was generated by serial dilutions of 20 $\mu \mathrm{M} \mathrm{H}_{2} \mathrm{O}_{2}$.

\section{$T$ cell activation}

T cell activation was measured as described (44). Briefly, $10^{5}$ splenocytes from DO11.10 (ovalbumin 323-339-specific, $I-A^{d}$-restricted), TS1 (hemagglutinin $110-119$-specific, $I-E^{d}$-restricted), Clone4 (hemagglutinin $518-526$-specific, $H$ - $2 K^{d}$-restricted), or OT1 (ovalbumin 257-264-specific, $H$-2 $K^{b}$-restricted) transgenic mice were cultured with their respective cognate peptides and varying concentrations of irradiated (20Gy) MDSC from the blood of 4T1 tumor-bearing mice. Catalase (1000 or $500 \mathrm{U} / \mathrm{ml}$; Sigma Aldrich), sodium pyruvate (5 or $2.5 \mu \mathrm{M}$; Sigma Aldrich), nor-NOHA (500 $\mu \mathrm{M}$; Calbiochem, CA), or L-NMMA (500 $\mu \mathrm{M}$; Calbiochem) were included in some assays. Reversal of suppression $=-100 \% \times\left[1-\left(\mathrm{CPM}_{\text {No Inhibitor }} / \mathrm{CPM}_{\text {Inhibitor }}\right)\right]$. For some experiments, $\mathrm{Nrf} 2^{+/+}$ MDSC were either initially cultured with splenocytes, or added after the addition of cognate peptide. Fresh MDSC were used for experiments in which MDSC were added to overnight splenocyte cultures. Cultures were pulsed with ${ }^{3} \mathrm{H}$-thymidine $(1 \mathrm{nCu} / 250 \mu \mathrm{L})$ on day 4 and harvested on day 5. Peptides were synthesized at the University of Maryland Baltimore (UMB) Biopolymer Core Facility.

\section{MDSC-macrophage cross-talk}

Peritoneal macrophages were prepared from tumor-free mice as described (35) and were $>95 \% \mathrm{CD} 11 \mathrm{~b}^{+} \mathrm{F} 4 / 80^{+}$cells as assessed by flow cytometry. MDSC and 
macrophage cross-talk experiments were performed as described (47). Supernatants were analyzed for IL-10 using ELISA kits (R\&D Systems and eBioscience, San Diego, CA) per the manufacture's protocol. NO was assayed by Griess assay as described (47).

\section{Statistical Analyses}

Student's $t$-test and Tukey's Honestly Significant Difference (HSD) test were performed using Microsoft Excel 2013. Values denoted with different letters (e.g. a, b, c, etc.) are significantly different from each other; values with the same letter are not significantly different. Tumor growth and ROS data were analyzed using the MannWhitney test on the www.VassarStats.net website. Survival data were analyzed using the log-rank test from the Walter and Eliza Hall Institute of Medical Research Bioinformatics webpage (http://bioinf.wehi.edu.au/software/russell/logrank/). Values of $\mathrm{p}<0.05$ were considered statistically significant. Values are \pm SD.

\section{Results}

\section{Host expression of $\mathrm{Nrf} 2$ enhances tumor progression}

The role of host-derived Nrf2 in tumor progression has been controversial. Some studies indicate that Nrf2 supports tumor growth (48-53), while other studies suggest it deters carcinogenesis (54-58). To determine whether host Nrf2 contributes to or deters tumor growth in BALB/c and C57BL/6 mice, $\mathrm{Nrf}^{+/+}$and $\mathrm{Nrf} 2^{-/-} \mathrm{BALB} / \mathrm{c}$ and $\mathrm{C} 57 \mathrm{BL} / 6$ mice were injected with syngeneic 4T1 mammary carcinoma or MC38 colon carcinoma, respectively, and followed for primary tumor growth (Figure 1A) and survival (Figure 1B). Nrf2 did not impact the growth rate of either primary tumor. However, tumor- 
bearing $\mathrm{Nrf}^{+/+}$mice had decreased mean survival times compared to $\mathrm{Nrf2}^{-/-}$mice (BALB/c: 42.2 vs 50.8 days; $0 \%$ and $27.27 \%$ of mice survived >100 days, respectively; C57BL/6: 35.8 vs. 43 days; $12.5 \%$ and $77.78 \%$ of mice survived $>50$ days, respectively), indicating that Nrf2 supports tumor progression in these mouse strains.

\section{Host expression of Nrf2 enhances MDSC suppressive potency and the quantity of tumor-infiltrating MDSC.}

If Nrf2 mediates its effects by increasing MDSC suppressive potency, then MDSC from tumor-bearing $\mathrm{Nrf}^{+/+}$mice will be more suppressive than MDSC from $\mathrm{Nrf}^{-/-}$mice. To test this possibility, titered quantities of MDSC from tumor-bearing $\mathrm{BALB} / \mathrm{c} \mathrm{Nrf2} 2^{+++}$and $\mathrm{Nrf2}^{-{ }^{-}}$mice were co-cultured with $\mathrm{T}$ cells from $\mathrm{TcR}$ transgenic mice plus cognate peptide, and the cultures assayed for T cell activation (Figure 2A, Supplemental Figure 2A). Both $\mathrm{CD} 4^{+}$and $\mathrm{CD} 8^{+} \mathrm{T}$ cells were suppressed more efficiently by $\mathrm{Nrf}^{+/+}$MDSC than by Nrf2 $2^{-/-}$MDSC, suggesting that Nrf2 drives the suppressive potency of MDSC. Since differences in MDSC viability may impact suppressive potency, we determined the kinetics of MDSC-mediated suppression (Supplemental Figure 2B) and then assessed the viability of MDSC at the time they would be active (Supplemental Figure 2C). Addition of MDSC to splenocyte plus cognate peptide cultures at or after $19 \mathrm{hrs}$ did not result in suppression, indicating that MDSC viability was only relevant at $\leq 16 \mathrm{hrs}$, although viability did not differ up to 24 hrs in culture. These data demonstrate that $\mathrm{Nrf2}^{+/+} \mathrm{MDSC}$ are more suppressive than $\mathrm{Nrf}^{---}$MDSC and that the difference is not due to differences in MDSC viability. MDSC use several mechanisms to inhibit T cells, such as the secretion of ROS, 
including $\mathrm{H}_{2} \mathrm{O}_{2}$, which decreases T cell expression of IL-2, IFN $\gamma$, and $\mathrm{CD} 3 \zeta(36,59)$, and the production of arginase which deprives $\mathrm{T}$ cells of the amino acid arginine, leading to $\mathrm{CD} 3 \zeta$ synthesis arrest (60). MDSC also sequester cysteine which is an essential amino acid for T cell activation (61). $\mathrm{Nrf}^{+/+}$MDSC secrete more $\mathrm{H}_{2} \mathrm{O}_{2}$ than $\mathrm{Nrf}^{-/-}$MDSC (Figure 2B). $\mathrm{H}_{2} \mathrm{O}_{2}$ contributes to the suppressive potency of MDSC since inclusion of the $\mathrm{H}_{2} \mathrm{O}_{2}$ scavengers catalase or sodium pyruvate in cultures of MDSC plus transgenic T cells plus cognate peptide, significantly increased T cell activation (Figure 2C, Supplemental Figure 2D). Neither $\mathrm{Nrf}^{+/+}$nor $\mathrm{Nrf}^{-/-}$MDSC produce nitric oxide (NO; Supplemental Figure 3A), and the NOS2 inhibitor L-NMMA did not rescue T cell activation in the presence of MDSC (Figure 2C, Supplemental Figure 2D). $\mathrm{Nrf}^{+/+}$and $\mathrm{Nrf}^{-/-}$MDSC both use arginase to suppress $\mathrm{T}$ cell activation since inclusion of the arginase inhibitor nor-NOHA restores T cell activation (Figure 2C, Supplemental Figure 2D), and there is no difference in their content of arginase (Supplemental Figure 3B). MDSC from both wild type and knockout mice express similar levels of $\mathrm{xCT}$, the chain of the dimeric $\mathrm{x}_{\mathrm{c}}{ }^{-}$transporter that regulates the uptake of cystine (Supplemental Figure 3C). These results indicate that $\mathrm{Nrf}_{2}^{+/+}$and $\mathrm{Nrf}^{-/-}$MDSC utilize similar mechanisms to suppress $\mathrm{T}$ cell activation and proliferation, but $\mathrm{Nrf}^{+/+} \mathrm{MDSC}$ are more suppressive because they produce more $\mathrm{H}_{2} \mathrm{O}_{2}$.

MDSC also promote tumor progression by down-regulating L-selectin on naïve $\mathrm{T}$ cells thereby preventing naïve $\mathrm{T}$ cell trafficking into lymph nodes (32), and they produce IL-10 which polarizes macrophages towards a tumor-promoting phenotype (35). MDSC from $\mathrm{Nrf}^{+/+}$and $\mathrm{Nrf}^{-/-}$tumor-bearing BALB/c mice equally down-regulated $\mathrm{T}$ cell $\mathrm{L}$ selectin (Supplemental Figure 3D) demonstrating that Nrf2 does not affect naïve T cell 
entry into lymph nodes. Using MDSC and macrophages from BALB/c IL- $10^{+/+}$and IL$10^{-/-}$mice, we previously demonstrated that in co-cultures of MDSC and macrophages, MDSC are the sole producers of IL-10, and that MDSC production of IL-10 is enhanced by macrophages $(35,47,62)$. Surprisingly, Nrf2 decreased MDSC production of IL-10, thus reducing the ability of MDSC to polarize macrophages towards a type 2 phenotype (Supplemental Figure 3E). These results indicate that Nrf2 does not impact the ability of MDSC to decrease T cell homing to lymph nodes, but does reduce the ability of MDSC to polarize macrophages towards a tumor-promoting phenotype.

Since MDSC are present in most solid tumors where they can exert pro-tumor activity (63), we assessed the proportion of MDSC in 4T1 primary tumors resected from BALB/c Nrf2 ${ }^{+/+}$and $\mathrm{Nrf}^{-{ }^{--}}$mice (Figure 2D). Tumors derived from Nrf2 $2^{-/-}$mice had significantly fewer MDSC compared to tumors from wild type littermates. However, the ratio of tumor-infiltrating PMN-MDSC to M-MDSC was the same in $\mathrm{Nrf}^{+/+}$and $\mathrm{Nrf2}^{-{ }^{--}}$ mice (Supplemental Figure 4A). The proportion of tumor-infiltrating CD11 $\mathrm{c}^{+}, \mathrm{CD} 4^{+}$, and $\mathrm{CD}^{+}$cells did not differ between the $\mathrm{Nrf}^{+/+}$and $\mathrm{Nrf}^{-/-}$mice, but tumors from $\mathrm{Nrf2}^{-}$ I- mice contained significantly more $\mathrm{F} 4 / 80^{+}$and B220 ${ }^{+}$cells (Figure 2D).

To determine if the differences in tumor-infiltrating MDSC were due to differences in MDSC trafficking, MDSC were isolated from the peripheral blood, bone marrow, and tumor of 4T1-bearing $\mathrm{Nrf}^{+/+}$and $\mathrm{Nrf2}^{-/}$mice and assayed for CCR2 and CXCR4, chemokine receptors that regulate MDSC migration $(64,65)$ (Supplemental Figure 4B). CCR2 and CXCR4 expression did not differ between $\mathrm{Nrf}^{+/+}$and $\mathrm{Nrf}^{-/-}$ MDSC. Likewise, $\mathrm{Nrf2}^{+/+}$and $\mathrm{Nrf2}^{-/-}$MDSC migrated at the same rate in a transwell chemotaxis assay (Supplemental Figure 4C), indicating that Nrf2 does not influence 
MDSC trafficking.

Collectively, these data suggest that Nrf2 decreases the survival time of tumorbearing mice by enhancing MDSC suppressive activity and by increasing the accessibility of MDSC to the TME.

\section{Nrf2 decreases MDSC oxidative stress and apoptosis}

Since the increased number of tumor-infiltrating MDSC in $\mathrm{Nrf}_{2}^{+/+}$mice was not due to enhanced MDSC trafficking, we speculated that MDSC in $\mathrm{Nrf}^{+/+}$mice had lower levels of intracellular ROS and therefore were less oxidatively stressed. Therefore, we assessed intracellular ROS levels in MDSC from 4T1-bearing $\mathrm{Nrf}^{+/+}$and $\mathrm{Nrf2}^{-{ }^{-}}$mice and in MDSC differentiated in vitro from bone marrow progenitor cells. MDSC in the blood of $\mathrm{Nrf}^{+/+} 4 \mathrm{~T} 1$-bearing mice had significantly lower levels of intracellular ROS compared to $\mathrm{Nrf2}^{-/-}$MDSC as measured by DCFDA fluorescence (Figure 3A), as did MDSC differentiated from bone marrow progenitor cells of $\mathrm{Nrf}^{+/+}$mice (Figure 3B). Therefore, Nrf2 reduces intracellular ROS, consistent with the concept that MDSC in $\mathrm{Nrf}^{+/+}$mice survive longer due to reduced oxidative stress.

To determine if reduced levels of oxidative stress in $\mathrm{Nrf}^{+/+} \mathrm{MDSC}$ correlate with reduced apoptosis, in vivo tumor-induced and in vitro differentiated MDSC were examined for apoptosis. Circulating MDSC from 4T1 tumor-bearing $\mathrm{Nrf}^{+/+}$and $\mathrm{Nrf} 2^{-/-}$ mice were stained for Gr1 and CD11b, and with Annexin V (Figure 4A). MDSC from $\mathrm{Nrf}^{+/+}$mice were $53 \%$ less apoptotic than MDSC from $\mathrm{Nrf}^{-/-}$mice. MDSC differentiated in vitro in bone marrow cultures were similarly analyzed except dead cells were excluded by 7AAD staining. In vitro differentiated MDSC from $\mathrm{Nrf} 2^{+/+}$mice were 
$29 \%$ less apoptotic compared to MDSC from Nrf2 ${ }^{-/-}$mice (Figure 4B), confirming the concept that reducing oxidative stress decreases apoptosis.

A decrease in apoptotic rate could result in an increase in circulating MDSC. This possibility was tested by comparing the levels of MDSC in the blood of tumor-bearing $\mathrm{Nrf}^{+/+}$vs. Nrf2 ${ }^{-/}$mice. Mice with the same tumor diameters were compared to eliminate MDSC differences due to different tumor burdens (Supplemental Figure 4D). There was no difference in the level of circulating MDSC in tumor-bearing $\mathrm{Nrf2}^{+/+}$and $\mathrm{Nrf2}^{-/-}$mice, indicating that the differential apoptotic rates did not impact MDSC accumulation in blood.

\section{Nrf2-deficiency increases the rate of MDSC generation in the bone marrow}

Since $\mathrm{Nrf2}^{-/-}$MDSC are more apoptotic than $\mathrm{Nrf}^{+/+}$MDSC, yet tumor-bearing $\mathrm{Nrf}_{2}^{+/+}$and $\mathrm{Nrf2}^{--}$mice have similar levels of circulating MDSC, we hypothesized that MDSC differentiate more rapidly in $\mathrm{Nrf2}^{-/-}$mice. To test this hypothesis, bone marrow cells from tumor-free $\mathrm{Nrf}^{+/+}$and $\mathrm{Nrf2}^{-/-}$mice were cultured under conditions to promote MDSC differentiation, and the number of resulting MDSC was quantified (Figure 5A). C57BL/6 and BALB/c Nrf2 ${ }^{-/-}$bone marrow produced $16 \%$ and $76 \%$ more MDSC than the corresponding $\mathrm{Nrf2}^{+/+}$bone marrow, respectively. The increases were due to the expansion of PMN-MDSC (Figure 5B). Therefore, Nrf2 reduces the rate of MDSC generation from bone marrow progenitor cells. Taken together with the apoptotic studies of Figure 4, these data indicate that circulating MDSC levels are maintained by a balance between the generation of MDSC in the bone marrow and their turn-over in the periphery. 


\section{Discussion}

For cells to survive the hostile TME, they must protect themselves against oxidative stress. Since Nrf2 regulates many genes that enable cells to survive oxidative stress, we examined the role of Nrf2 in the maintenance of MDSC suppressive activity, survival, and presence in solid tumors. Nrf2 enhanced MDSC suppressive activity by increasing MDSC production of $\mathrm{H}_{2} \mathrm{O}_{2}$, and increased the quantity of tumor-infiltrating MDSC by reducing their oxidative stress and apoptotic rate. Nrf2 did not affect circulating levels of MDSC in tumor-bearing mice since the decreased apoptotic rate of tumor-infiltrating MDSC was balanced by a decreased rate of differentiation from bone marrow progenitor cells. Collectively, these results provide a new avenue by which Nrf2 regulates tumor progression and add Nrf2 to the list of genes that govern MDSC accumulation, survival, and function.

The TME is an inflamed milieu that includes multiple cell types (e.g. tumor cells, MDSC, macrophages, dendritic cells, lymphocytes, mast cells, neutrophils cancerassociated fibroblasts, etc.). These cells participate in a complex crosstalk network that regulates the production of inflammatory mediators (47). Nrf2 increases the number of tumor-infiltrating MDSC and therefore enhances the opportunity for crosstalk between MDSC and other tumor-resident cells. Our previous studies demonstrated that macrophages enhance MDSC production of IL-10 which in turn polarizes macrophages towards a tumor-promoting phenotype $(35,47)$. Since Nrf2-deficiency increases both macrophage-dependent and macrophage-independent IL-10 production by MDSC, 
strategies aimed at limiting Nrf2 may facilitate the development of pro-tumor macrophages.

Pharmacologic down-regulation of Nrf2 in MDSC may decrease the quantity of tumor-infiltrating MDSC and their suppressive potency. However, it will not reduce the level of circulating MDSC due to the homeostatic compensation by increased generation of MDSC from bone marrow progenitor cells. If MDSC predominantly mediate their suppressive effects on $\mathrm{T}$ cells within the tumor, then Nrf2 down-regulation may reduce MDSC-mediated suppression. However, MDSC may also mediate their effects on T cells in the periphery by suppressing circulating tumor-reactive T cells. In addition, MDSC are known to prevent the entry of naïve T cells into lymph nodes where they could become activated $(32,45)$. Therefore, down-regulation of Nrf2 in MDSC may only marginally reduce immune suppression and improve anti-tumor immunity because levels of circulating MDSC will remain constant due to increased generation of MDSC from bone marrow progenitor cells.

It is unlikely that the homeostatic balance of MDSC in tumor-bearing individuals is achieved by Nrf2 directly regulating genes that drive MDSC generation. Antibodymediated depletion of MDSC results in the rebound of MDSC to levels that are higher than pre-depletion levels (66), demonstrating that some type of feed-back mechanism regulates MDSC homeostasis. Since Nrf2, like antibody-mediated depletion, alters extramedullary levels of MDSC, Nrf2 most likely also regulates MDSC homeostasis via a feedback loop rather than by a direct effect on genes within bone marrow progenitor cells.

Given that homeostasis maintains a constant level of circulating MDSC in 
individuals with tumor, monotherapies aimed at reducing MDSC levels by targeting circulating MDSC are unlikely to be effective. In contrast, strategies that target the induction of MDSC from progenitor cells have the potential to interrupt homeostatic regulation and thereby reduce MDSC levels. Many inducers of MDSC have been identified. These are predominantly pro-inflammatory molecules $(67,68)$. Since these molecules are redundant and compensate for each other in their ability to drive MDSC generation, it will be necessary to develop inhibitors that cover the full range of inducers.

Whether the depletion of MDSC by their differentiation into macrophages or other myeloid cells also results in the replacement of immune suppressive MDSC by homeostasis is unknown. However, if this process does not increase the differentiation of MDSC from bone marrow progenitors, then promoting MDSC differentiation may be therapeutic. Indeed, drugs such as CpG motifs (69), all-trans retinoic acid (70), tetrabromocinnamic acid (71), and Vitamin D3 (72) that drive the differentiation of MDSC to more mature cells have shown therapeutic effects. Interestingly, all-trans retinoic acid is a known Nrf2 inhibitor (73), suggesting that there is interplay between MDSC differentiation and Nrf2 activity.

Although inhibition of Nrf2 in MDSC may not by itself impact tumor growth, combined effects of Nrf2 inhibitors on MDSC and tumor cells may be more effective since Nrf2 also impacts other cells. For example, inhibition of Nrf2 in dendritic cells enhances MHC II and CD86 expression (74), which could result in improved antigenpresentation and therefore better activation of tumor-reactive T cells. Additionally, Nrf2 activation in tumor cells increases tumor cell proliferation and resistance to chemotherapy and radiotherapy, thereby promoting tumor progression (reviewed in (75)). 
Since MDSC accumulation is positively correlated with tumor burden, treatment strategies that combine Nrf2 inhibitors with conventional chemotherapy and radiotherapy could decrease tumor burden and thereby indirectly reduce MDSC levels and increase anti-tumor immunity. 


\section{Figures}

\section{Figure 1}

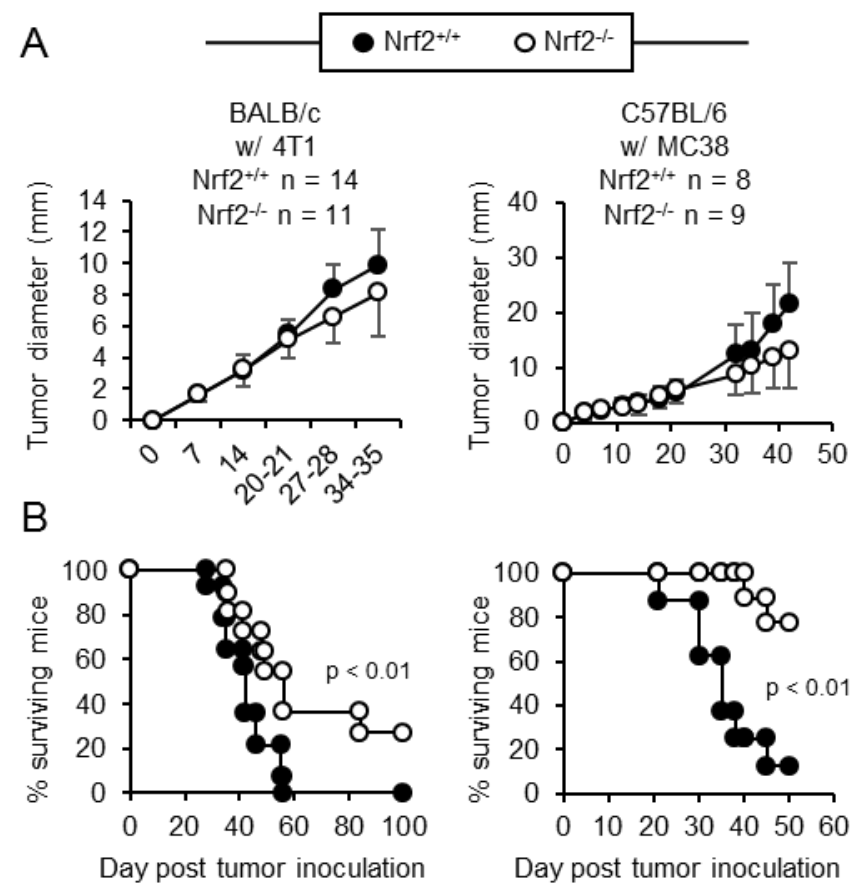

Figure 1: Nrf2 decreases survival time of tumor-bearing mice. $\mathrm{Nrf}^{+/+}$and $\mathrm{Nrf}^{-/-}$mice on the BALB/c (left panels) or C57BL/6 (right panels) backgrounds were injected with 4T1 mammary carcinoma or MC38 colon carcinoma, respectively. Mice were followed weekly for primary tumor growth (A) and survival time (B). Tumor diameter was calculated as the average measurement of tumor length and width. Data were pooled from two independent experiments. Tumor growth and survival time were tested for statistical significance by Mann-Whitney and log-rank test, respectively. 
Figure 2

A
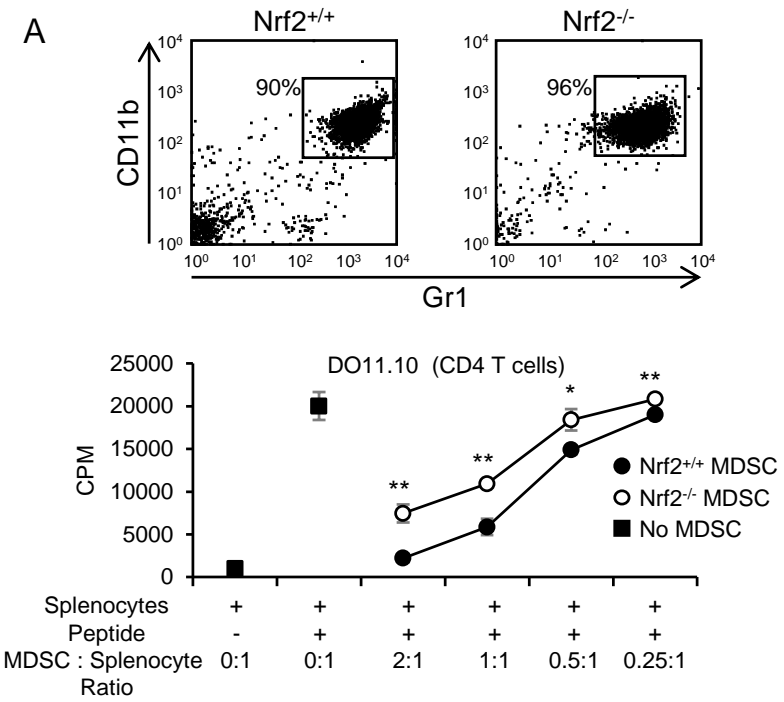

B

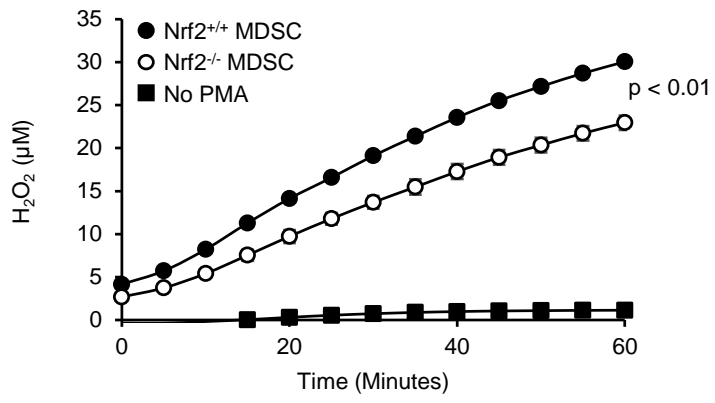

C

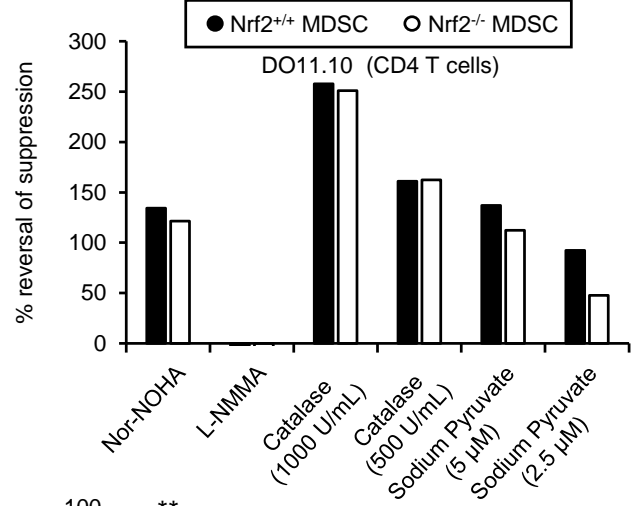

D

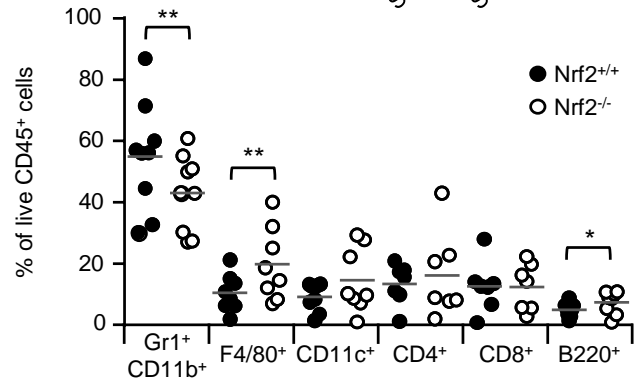


Figure 2: Nrf2 enhances MDSC suppressive activity and the quantity of tumorinfiltrating MDSC. (A) Nrf2 enhances MDSC-mediated CD4 ${ }^{+} \mathrm{T}$ cell suppression. MDSC from the peripheral blood of 4T1-bearing BALB/c Nrf2 $2^{+/+}$and $\mathrm{Nrf}^{-/-}$mice were assayed for their ability to suppress the antigen-activation of transgenic CD4 ${ }^{+}$(DO11.10) T cells. (B) Nrf2 enhances MDSC production of $\mathrm{H}_{2} \mathrm{O}_{2}$. MDSC from BALB/c Nrf2 ${ }^{+/+}$and $\mathrm{Nrf}^{-/-}$mice with 4T1 tumors were incubated with Amplex Red reagent, stimulated with PMA, and assayed for $\mathrm{H}_{2} \mathrm{O}_{2}$ production over time. (C) $\mathrm{Nrf}^{+/+}$and $\mathrm{Nrf}^{-/-}$MDSC suppress $\mathrm{CD}^{+} \mathrm{T}$ cell activation by producing arginase and $\mathrm{H}_{2} \mathrm{O}_{2}$. MDSC from the peripheral blood of 4T1-bearing BALB/c Nrf2 $2^{+/+}$and $\mathrm{Nrf}^{-/-}$mice were assayed for their ability to suppress the antigen-activation of transgenic $\mathrm{CD}^{+}(\mathrm{DO} 11.10) \mathrm{T}$ cells in the presence of nor-NOHA, L-NMMA, catalase, and sodium pyruvate. (D) Nrf2 enhances the quantity of tumor-infiltrating MDSC. Each circle represents an individual mouse. Figures A, B, and D were analyzed by Students $t$ test, Mann-Whitney test, and Wilcoxonrank sign test, respectively. Figures A, B, and C represent one of two experiments, each with one $\mathrm{Nrf}^{+/+}$and one $\mathrm{Nrf}^{-/-}$mouse per experiment. Data from figure D were pooled from 5 independent experiments; $* * \mathrm{p}<.01,{ }^{*} \mathrm{p}<.05$. 


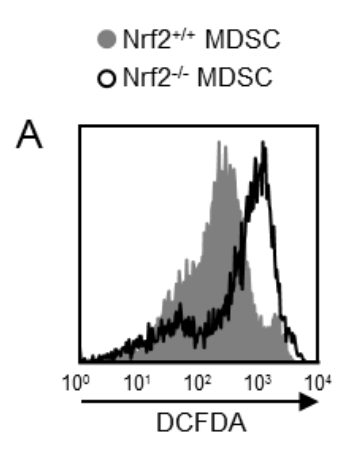

\section{Figure 3}
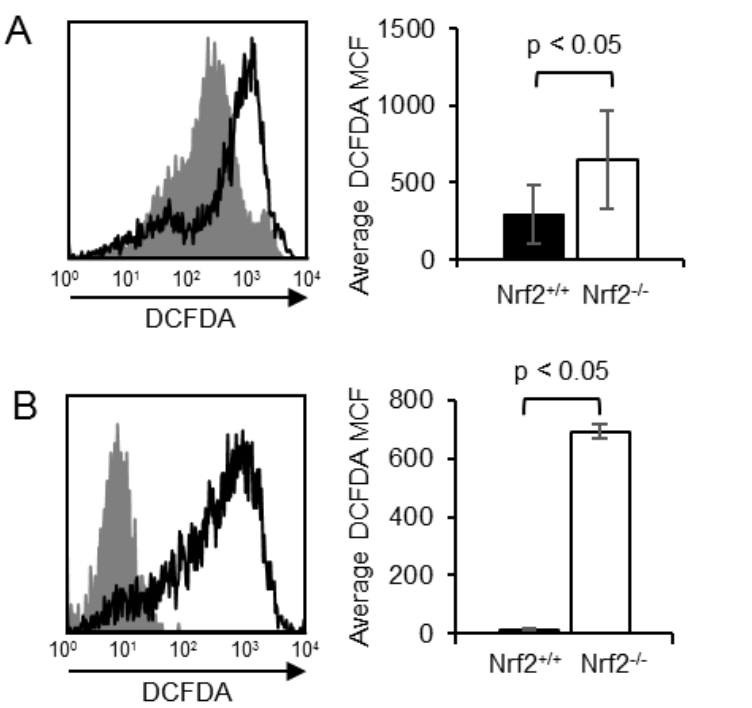

Figure 3: Nrf2 decreases intracellular MDSC oxidative stress. (A) Nrf2 decreases intracellular ROS in circulating MDSC of tumor-bearing mice. Circulating MDSC were harvested from 4T1-bearing BALB/c Nrf2 $2^{+/+}$and $\mathrm{Nrf}^{-/-}$mice, stained with DCFDA, and for $\mathrm{Gr} 1$ and $\mathrm{CD} 11 \mathrm{~b} . \mathrm{Gr} 1^{+} \mathrm{CD} 11 \mathrm{~b}^{+}$cells were gated and analyzed by flow cytometry for DCFDA fluorescence. Left histogram: DCFDA staining of MDSC from representative individual $\mathrm{Nrf}^{+/+}$and $\mathrm{Nrf}^{-{ }^{--}}$mice; right graph: average MCF of DCFDA staining for MDSC from six Nrf2 $2^{+/+}$and five $\mathrm{Nrf}^{-/-}$mice (B) Nrf2 decreases intracellular ROS in MDSC differentiated in vitro from bone marrow progenitor cells. Bone marrow cells from tumor-free BALB/c Nrf2 $2^{+/+}$or $\mathrm{Nrf}^{-/-}$mice were cultured for four days with IL-6 and GM-CSF, and the resulting cells were harvested, and stained with 7AAD and DCFDA, and for Gr1 and CD11b. 7AAD $\mathrm{Gr}^{+} \mathrm{CD}^{-} 11 \mathrm{~b}^{+}$cells were gated and analyzed by flow cytometry for DCFDA fluorescence. Left histogram: DCFDA staining for bone marrow differentiated MDSC from individual $\mathrm{Nrf}^{+/+}$and $\mathrm{Nrf} 2^{-/-}$mice; right graph: average MCF of DCFDA in bone marrow differentiated MDSC from three $\mathrm{Nrf}^{+/+}$and three $\mathrm{Nrf}^{-/-}$mice. Data were tested for statistical significance by Student's $t$ test. 


\section{Figure 4}
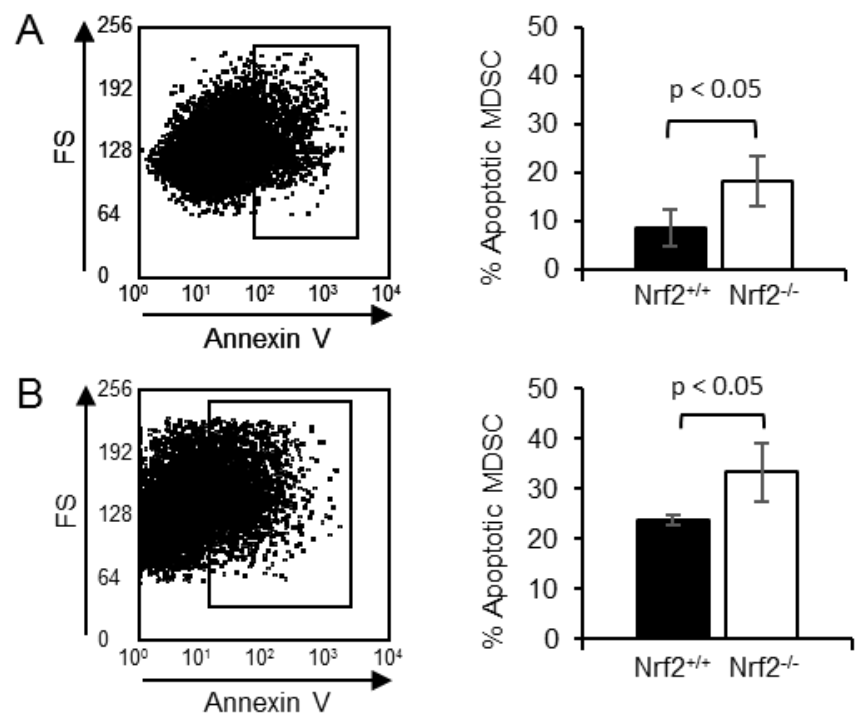

Figure 4: Nrf2 protects MDSC from apoptosis. (A) Nrf2 decreases apoptosis in circulating MDSC of tumor-bearing mice. Circulating MDSC were harvested from 4T1bearing BALB/c Nrf2 $2^{+/+}$and $\mathrm{Nrf}^{-/-}$mice, and stained for Gr1, CD11b, and with Annexin $\mathrm{V}$ and propidium iodide (PI) or 7AAD, and analyzed by flow cytometry. Live $\mathrm{Gr}^{+} \mathrm{CD} 11 \mathrm{~b}^{+} \mathrm{MDSC}\left(\mathrm{PI}^{-}\right.$or $\left.7 \mathrm{AAD}^{-}\right)$were gated and assessed for Annexin V. (B) Nrf2 decreases apoptosis in MDSC differentiated in vitro from bone marrow progenitor cells. MDSC derived from bone marrow cell cultures were harvested and stained and stained for Gr1, CD11b, and with Annexin V and propidium iodide (PI) or 7AAD, and analyzed by flow cytometry. Live $\mathrm{Gr} 1^{+} \mathrm{CD} 11 \mathrm{~b}^{+} \mathrm{MDSC}\left(\mathrm{PI}^{-}\right.$or $\left.7 \mathrm{AAD}^{-}\right)$were gated and assessed for Annexin V. Live $\mathrm{Gr}^{+} \mathrm{CD} 11 \mathrm{~b}^{+} \mathrm{MDSC}\left(\mathrm{PI}^{-}\right.$or $\left.7 \mathrm{AAD}^{-}\right)$were gated and assessed for Annexin V. Left panels: representative staining of Annexin V. Right graphs: average percent of live Annexin $\mathrm{V}^{+} \mathrm{Gr} 1^{+} \mathrm{CD} 11 \mathrm{~b}^{+}$cells. For bone marrow MDSC, data represent one of three experiments with each experiment using one $\mathrm{Nrf}^{+/+}$and one $\mathrm{Nrf} 2^{-/-}$mouse. For 4T1-derived MDSC, Nrf2 $2^{+/+}: n=6$ and $\mathrm{Nrf}^{-/-}: \mathrm{n}=5$. Data were tested for statistical significance using Student's $t$ test. 
Figure 5

\begin{tabular}{|c|c|c|c|c|c|c|}
\hline & \multicolumn{3}{|c|}{ Pre-culture } & \multicolumn{3}{|c|}{ Post-culture } \\
\hline & \# of cells & $\%$ MDSC & \# of MDSC & \# of cells & $\%$ MDSC & \# of MDSC \\
\hline $\mathrm{Nrf2}+/+(\mathrm{C} 57 \mathrm{BL} / 6)$ & 420,000 & 57.50 & 241,500 & $3,860,000$ & 92.79 & $3,581,694$ \\
\hline $\mathrm{Nrf2}^{-1-}(\mathrm{C} 57 \mathrm{BL} / 6)$ & 420,000 & 61.00 & 256,200 & $4,840,000$ & 95.53 & $4,623,652$ \\
\hline $\mathrm{Nrf2}^{+/+}(\mathrm{BALB} / \mathrm{c})$ & 420,000 & 36.17 & 151,914 & $1,150,000$ & 56.63 & 651,245 \\
\hline $\mathrm{Nr}+2^{-/}(\mathrm{BALB} / \mathrm{c})$ & 420,000 & 42.17 & 177,114 & $1,920,000$ & 50.93 & 977,856 \\
\hline
\end{tabular}
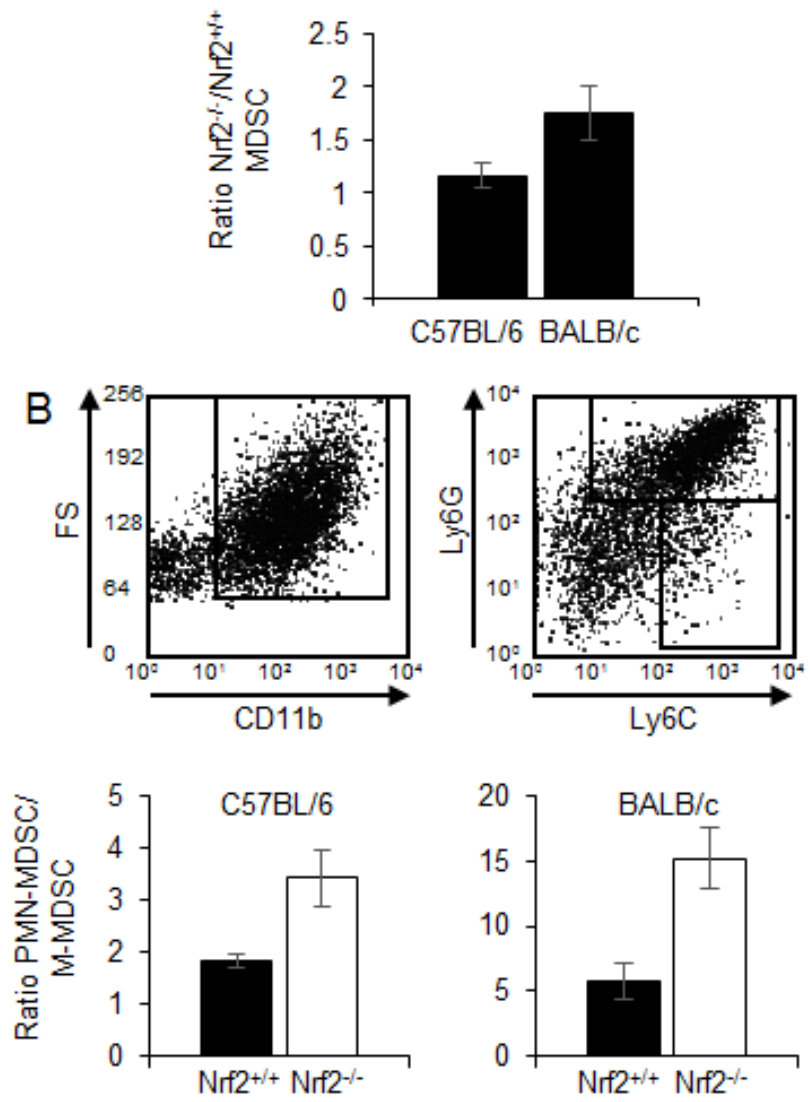

Figure 5: Nrf2 deficiency enhances MDSC proliferation. MDSC were differentiated in vitro from the bone marrow of tumor-free $\mathrm{Nrf}^{+/+}$and $\mathrm{Nrf}^{-/-} \mathrm{BALB} / \mathrm{c}$ and $\mathrm{C} 57 \mathrm{BL} / 6$ mice. The resulting cells were harvested, counted, and stained for Ly6G, Ly6C, and CD11b, and analyzed by flow cytometry. PMN-MDSC and M-MDSC were identified as $\mathrm{LyG}^{+} \mathrm{Ly}^{-/ / \mathrm{low}} \mathrm{CD} 11 \mathrm{~b}^{+}$and $\mathrm{Ly} 6 \mathrm{G}^{-/ \mathrm{low}} \mathrm{Ly} 6 \mathrm{C}^{+} \mathrm{CD} 11 \mathrm{~b}^{+}$cells, respectively. (A) Top: Quantity of total cells, percent of cells that are MDSC, and absolute number of MDSC 
pre-culture and after in vitro differentiation (post-culture). Data are representative of one of three independent experiments with one $\mathrm{Nrf}^{+/+}$and one $\mathrm{Nrf}^{-/-}$mouse per experiment. Bottom: Ratio of $\mathrm{Nrf2}^{-/-}$to $\mathrm{Nrf}^{+/+}$MDSC from the three independent experiments. A value $>1$ indicates that there is more proliferation in the absence of $\mathrm{Nrf} 2$. (B) $\mathrm{Nrf} 2$ deficiency preferentially enhances differentiation of PMN-MDSC from bone marrow progenitor cells. MDSC of panel A were gated and analyzed for PMN-MDSC and MMDSC. Top: Representative staining of M-MDSC and PMN-MDSC from individual $\mathrm{Nrf}^{+/+}$and $\mathrm{Nrf}^{-/-}$mice. Bottom: Average ratio of PMN-MDSC to M-MDSC from the three independent experiments. 
B

\begin{tabular}{lll}
\hline Gene & \multicolumn{1}{c}{ Forward } & \multicolumn{1}{c}{ Reverse } \\
\hline L32 & 5'- CTGCCATCTGTTTTACGGCA & 5'- ATCAGGATCTGGCCCTTGAAC \\
NQO1 & 5'- CGCCTGAGCCCAGATATTGT & 5'- GCACTCTCTCAAACCAGCCT \\
Catalase & 5'- CACTGACGAGATGGCACACT & 5'- TGTGGAGAATCGAACGGCAA \\
HO-1 & 5'- CCTCACAGATGGCGTCACTT & 5'- TGGGGGCCAGTATTGCATTT \\
GCLM & 5'- TGGAGTTCCCAAATCAGCCC & 5'- CAACTCCAAGGACGGAGCAT \\
NRF2 & 5'- TGGACGGACTATTGAAGGCTG & 5'- ATCAGGATCTGGCCCTTGAAC \\
Lac Z & 5'- GCGGATTGACCGTAATGGGATAGG & \\
\hline
\end{tabular}

C
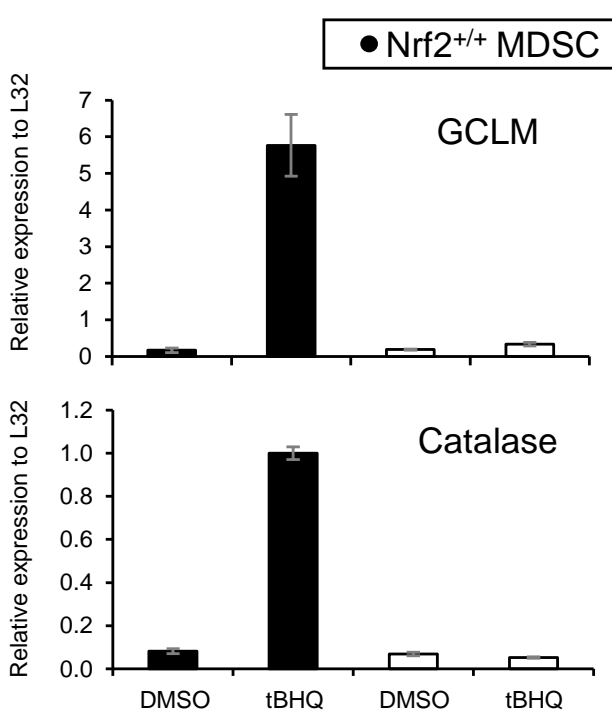

o $\mathrm{Nrf2}^{-/-\mathrm{MDSC}}$
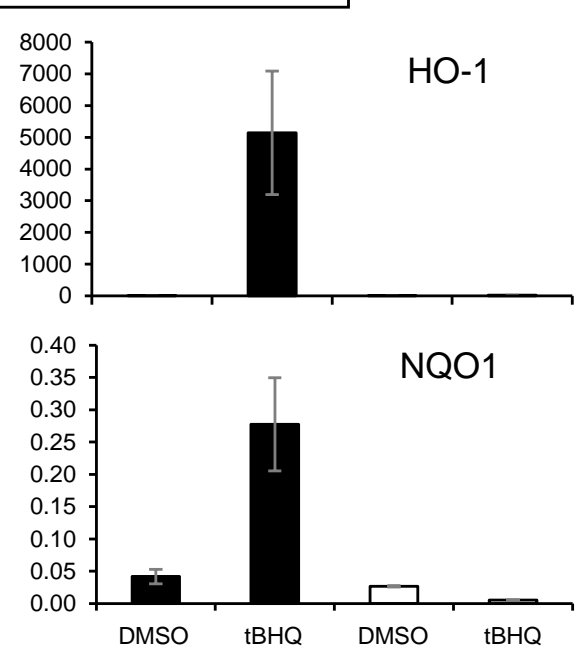

Supplemental Figure 1. MDSC from Nrf2-/- mice are deficient for Nrf2 and do not upregulate Nrf2-regulated genes in response to stress. (A) Genomic DNA was isolated from the of blood of offspring from Nrf2+/- $\times$ Nrf2+/- matings and assessed for disruption in the Nrf2 gene. BALB/c Nrf2+/+ mice are identified by a 734 bp band; Nrf2-- mice by a 400 bp band. All offspring from the BALB/c $\mathrm{Nrf2}^{+-} \times \mathrm{Nrf2}^{+-}$matings were analyzed. (B) Primers used in $\mathrm{qPCR}$ and PCR to type BALB/C Nrf2+$x$ Nrf2 ${ }^{+-}$offspring (Nrf2 and LacZ primers) and to assess expression of Nrf2-regulated genes (L32, NQO1, catalase, HO-1, and GCLM primers). (C) MDSC from Nrf2-deficient mice do not upregulate Nrf2-regulated genes. Tumor-induced MDSC were stressed with $\mathrm{tBHQ}$ and assessed by qPCR for GCLM, HO-1, catalase, and NQO1. Data are representative of two independent experiments. 


\section{Supplemental Figure 2}

A

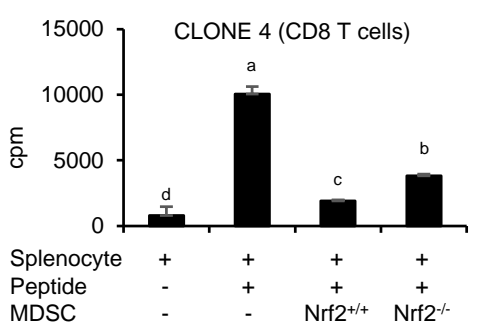

B
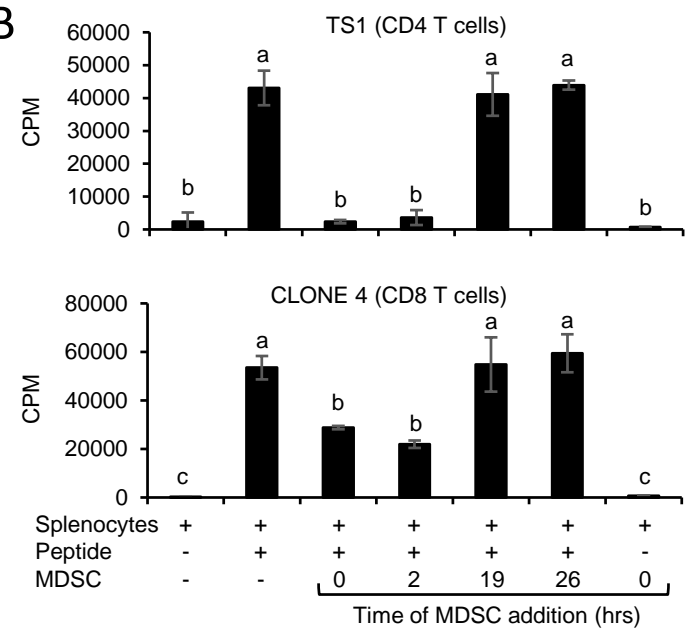

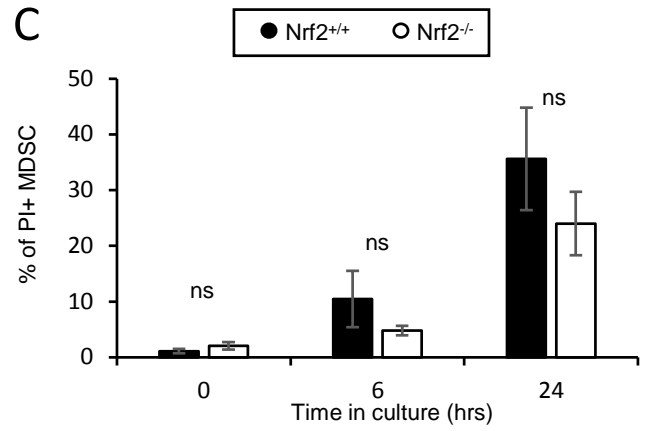

D

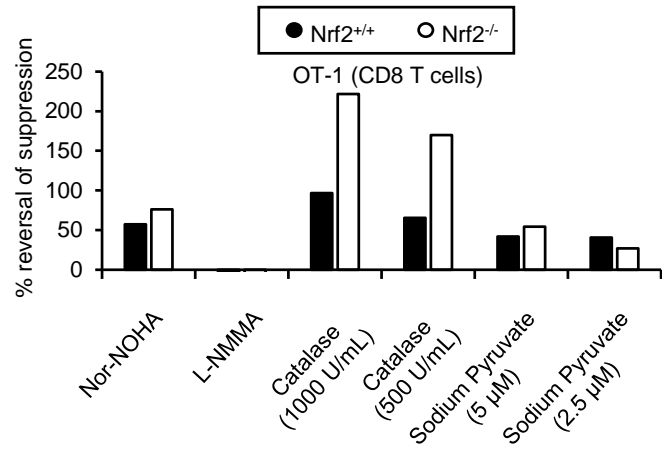

Supplemental Figure 2: Nrf2 enhances MDSC-mediated suppression of T cells. (A) Nrf2-MDSC are less suppressive than $\mathrm{Nrf2}^{+++}$MDSC for antigen-activated transgenic CD8 ${ }^{+} \mathrm{T}$ cells. MDSC from $\mathrm{Nrf2}^{-/}$and $\mathrm{Nrf}^{+/+}$mice were cultured with splenocytes from Clone 4 transgenic mice plus cognate peptide and analyzed for T cell activation. (B) MDSC suppress T cell activation early during the in vitro suppression assay. MDSC from the peripheral blood of $4 \mathrm{~T} 1$-bearing BALB/c Nrf2 ${ }^{+/}$mice were assayed for their ability to suppress the antigen-activation of transgenic CD4 $4^{+}$(TS1) and $\mathrm{CD}^{+}$ (Clone) T cells. MDSC were added to splenocyte cultures at a 1:1 ratio at the start of the culture $(0$ hrs) or at various times after the addition of cognate peptide (2, 19, and $26 \mathrm{hrs})$. Data were analyzed for statistical significance by Tukey HSD test, and are representative of one of three independent experiments. (C) Nrf2 does not impact MDSC viability in culture. Gr1 ${ }^{+} \mathrm{CD} 11 \mathrm{~b}{ }^{+}$cells in the peripheral blood of 4T1-bearing BALB/c Nrf2+/+ and $\mathrm{Nrf}^{-/-}$mice were cultured for varying periods, harvested and stained with $\mathrm{PI}$, and assessed for viability by flow cytometry. Data were tested for statistical significance by Student's $t$ test, and are from one of two independent experiments with three $\mathrm{Nrf2}^{+/+}$ and three $\mathrm{Nrf2}^{-/}$mice per experiment. (D) $\mathrm{Nrf}^{+/+}$and $\mathrm{Nrf2}^{-/-}$MDSC suppress $\mathrm{CD} 4^{+} \mathrm{T}$ cell activation by producing arginase and $\mathrm{H}_{2} \mathrm{O}_{2}$. MDSC from $\mathrm{Nrf2}^{--}$and $\mathrm{Nrf2}^{+++}$mice were cultured with splenocytes from OT-1 transgenic mice plus cognate peptide in the presence or absence of the arginase inhibitor nor-NOHA or the $\mathrm{H}_{2} \mathrm{O}_{2}$ scavengers catalase or sodium pyruvate. Data are from one of two independent experiments. 


\section{Supplemental Figure 3}

A

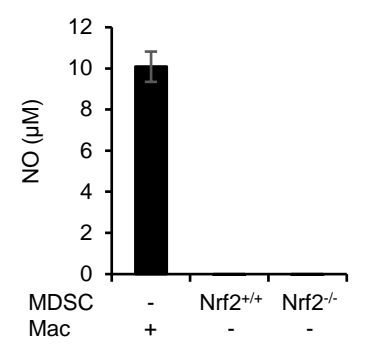

B

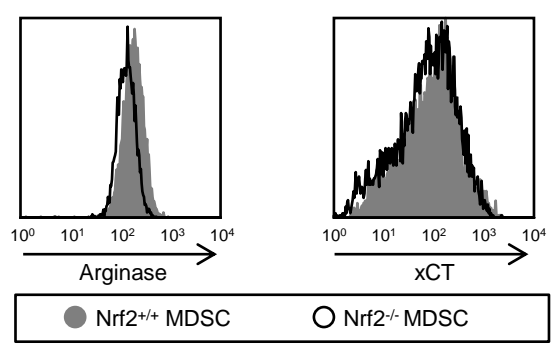

D

$\mathrm{Nrf}^{+/+}$

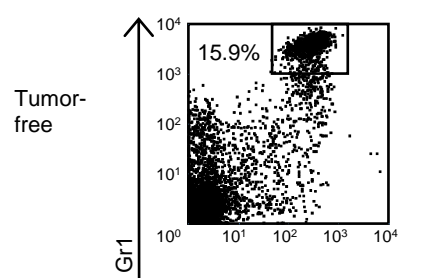

ডั

Tumor-
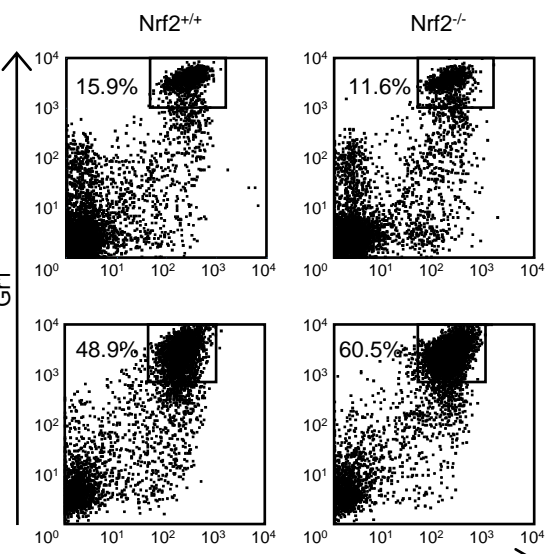

Tumor-bearing $\mathrm{O}$ Tumor-free

bearing
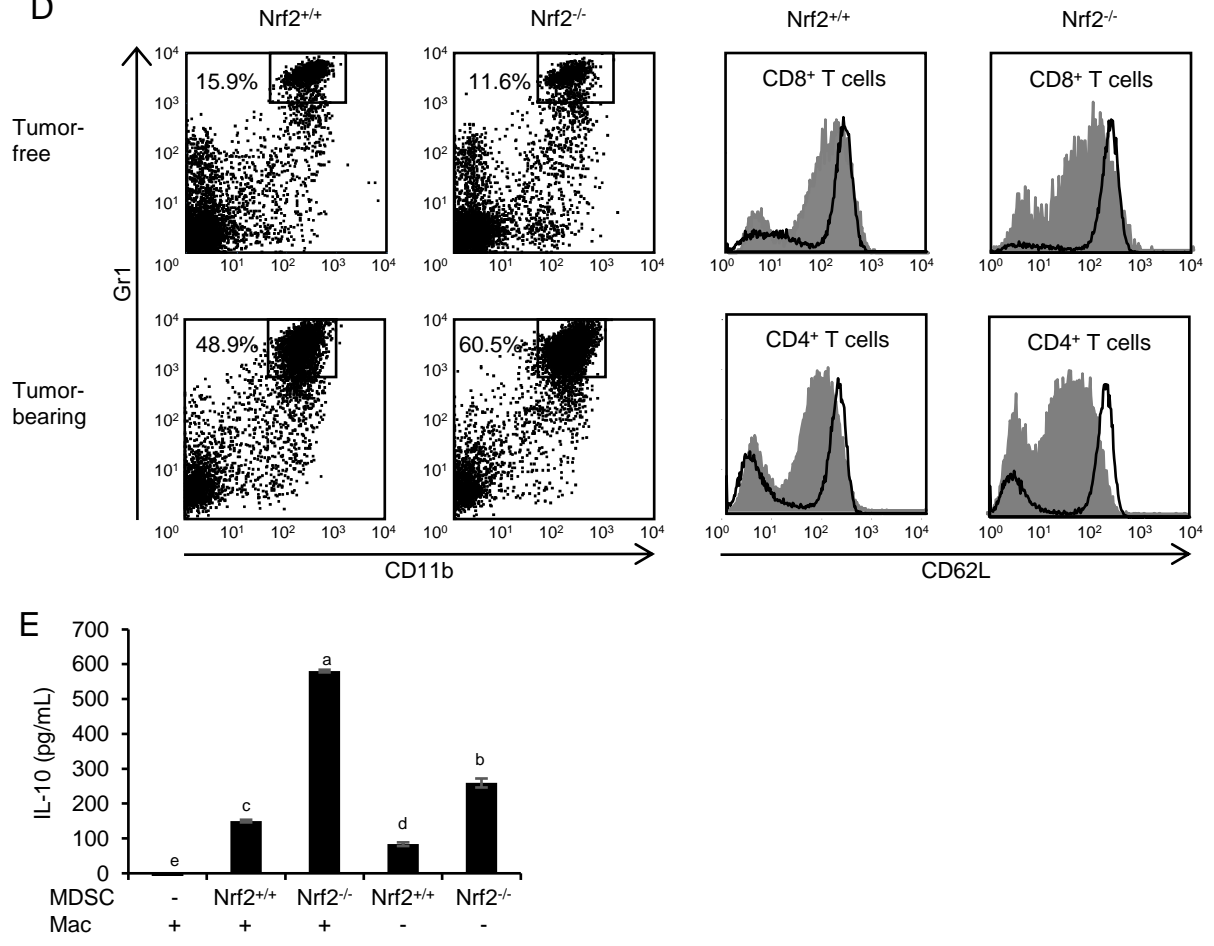

Supplemental Figure 3: Nrf2 does not impact MDSC production of NO, expression of Arg1 or xCT, or the down-regulation of L-selectin, but decreases MDSC production of IL-10. (A) Nrf2 has no impact on MDSC expression of NO. MDSC from the blood of BALB/c Nrf2 ${ }^{+/+}$and Nrf2 ${ }^{-/} 4 \mathrm{~T} 1$ tumor-bearing mice, and peritoneal macrophages were cultured and supernatants were assayed by Greiss assay for NO. (B) Nrf2 has no effect on MDSC expression of arginase or (C) XCT. MDSC from the peripheral blood of BALB/c mice with 4T1 tumors were stained for Arg1 and xCT. (D) Nrf2 does not impact MDSC-mediated down-regulation of L-selectin on naïve T cells. Cells were harvested from the peripheral blood of tumor-free BALB/c and 4T1-bearing $\mathrm{Nrf}^{+/+}$and $\mathrm{Nrf}^{-/-}$mice and stained with 7AAD and for CD3, CD4, CD8, CD11b, CD62L, and $\mathrm{Gr} 1$, and analyzed by flow cytometry. (E) Nrf2 decreases MDSC production of IL-10. MDSC from the blood of BALB/c Nrf2+/+ and Nrf2-- 4T1 tumor-bearing mice were cultured with or without peritoneal macrophages, and supernatants were assayed by ELISA for IL-10. Data in E were analyzed by Tukey HSD test. Data for panels A-E are from one mouse per group and are representative of 2, 2, 2, 4, and 2 experiments, respectively, with one $\mathrm{Nrf}^{+/+}$and one $\mathrm{Nrf2}^{-/-}$mouse per independent experiment. 


\section{Supplemental Figure 4}
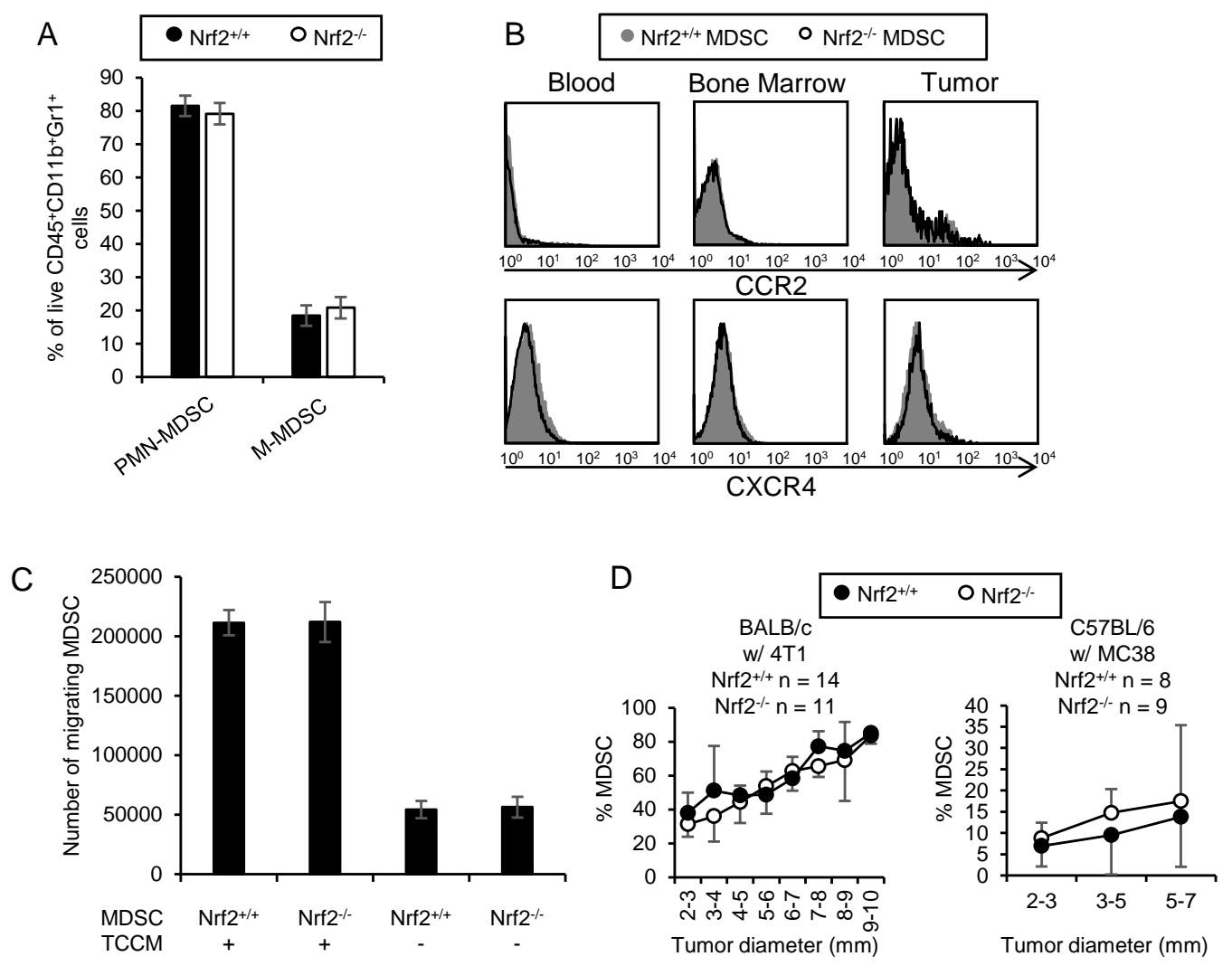

Supplemental Figure 4: Nrf2 does not alter the ratio of tumor-infiltrating PMN-MDSC to MMDSC or MDSC chemotaxis. (A) Nrf2 does not impact the percentage of tumor-infiltrating PMNMDSC or M-MDSC. Tumor infiltrating cells from 4T1 primary tumors of BALB/c Nrf2 $2^{+/+}$and $\mathrm{Nrf2}^{-/-}$ mice were analyzed by flow cytometry for \% live PMN-MDSC (7AAD-CD45+CD11b+Ly6G+Ly6C ${ }^{+}$ed and M-MDSC (7AAD-CD45 ${ }^{+}$CD11 b + Ly6G ${ }^{\text {low }}$ Ly6 $\left.C^{\text {hi }}\right)$. Data are the average of five $\mathrm{Nrf2}^{+/+}$and five Nrf2 ${ }^{-1}$ BALB/c mice. (B) Nrf2 does not impact MDSC expression of CCR2 or CXCR4. Cells were harvested from the blood, bone marrow, and tumor of 4T1-bearing $\mathrm{Nrf2}^{+/+}$and $\mathrm{Nrf2}^{--}$mice and stained with 7AAD and for CCR2, CXCR4, CD11b, CD45 and Gr1. 7AAD-CD45+Gr1+CD11 b+ cells were gated and analyzed for CCR2 and CXCR4. (C) Nrf2 does not affect MDSC migration. $500 \mu \mathrm{l}$ control medium [500 $\mathrm{\mu l}$ IMDM with 3\% Fetal Clone I, 1\% penicillin-streptomycin, $1 \%$ glutamax, $0.1 \%$ gentamycin], or migration medium [control media without serum and supplemented with $30 \%$ tumorconditioned media] were placed in the wells of 24 well plates. Transwells $(8 \mu \mathrm{M}$ polycarbonate semipermeable membranes; Corning Life Sciences, Union City, CA) containing $1 \times 10^{6}$ MDSC from the peripheral blood of $4 \mathrm{~T} 1$-bearing $\mathrm{Nrf2}^{+/+}$and $\mathrm{Nrf2}^{-/-}$mice in $100 \mu \mathrm{L}$ of control media were inserted into each well. Cultures were incubated $\left(37^{\circ} \mathrm{C}, 5 \% \mathrm{CO}_{2}\right)$ for 3 hours, after which the cells in the bottom chamber were counted. Samples were plated in triplicate and each replicate was counted twice. Data from B and C are representative of 3 and 2 independent experiments, respectively. (D) Nrf2 does not impact the level of circulating MDSC in tumor-bearing mice. BALB/c and C57BL/6 $\mathrm{Nrf2}^{+/+}$and $\mathrm{Nrf2}^{-/-}$mice were injected with $4 \mathrm{~T} 1$ and MC38 colon carcinoma, respectively. At weekly intervals, tumor diameters were recorded and mice were assessed for the percent of MDSC in the blood. Data were pooled from two independent experiments. 


\section{References}

1. Liou, G. Y., and P. Storz. 2010. Reactive oxygen species in cancer. Free Radic Res 44: 479-496.

2. McMahon, M., K. Itoh, M. Yamamoto, S. A. Chanas, C. J. Henderson, L. I. McLellan, C. R. Wolf, C. Cavin, and J. D. Hayes. 2001. The Cap'n'Collar basic leucine zipper transcription factor Nrf2 (NF-E2 p45-related factor 2) controls both constitutive and inducible expression of intestinal detoxification and glutathione biosynthetic enzymes. Cancer Res 61: 3299-3307.

3. Niture, S. K., and A. K. Jaiswal. 2012. Nrf2 protein up-regulates antiapoptotic protein Bcl-2 and prevents cellular apoptosis. J Biol Chem 287: 9873-9886.

4. Juhasz, A., Y. Ge, S. Markel, A. Chiu, L. Matsumoto, J. van Balgooy, K. Roy, and J. H. Doroshow. 2009. Expression of NADPH oxidase homologues and accessory genes in human cancer cell lines, tumours and adjacent normal tissues. Free Radic Res 43: 523-532.

5. Mohler, J., J. W. Mahaffey, E. Deutsch, and K. Vani. 1995. Control of Drosophila head segment identity by the bZIP homeotic gene cnc. Development 121: 237247.

6. Chan, J. Y., X. L. Han, and Y. W. Kan. 1993. Isolation of cDNA encoding the human NF-E2 protein. Proc Natl Acad Sci U S A 90: 11366-11370.

7. Moi, P., K. Chan, I. Asunis, A. Cao, and Y. W. Kan. 1994. Isolation of NF-E2related factor 2 (Nrf2), a NF-E2-like basic leucine zipper transcriptional activator 
that binds to the tandem NF-E2/AP1 repeat of the beta-globin locus control region. Proc Natl Acad Sci U S A 91: 9926-9930.

8. Kang, M. I., A. Kobayashi, N. Wakabayashi, S. G. Kim, and M. Yamamoto. 2004. Scaffolding of Keap1 to the actin cytoskeleton controls the function of Nrf2 as key regulator of cytoprotective phase 2 genes. Proc Natl Acad Sci U S A 101: 2046-2051.

9. Nguyen, T., P. J. Sherratt, H. C. Huang, C. S. Yang, and C. B. Pickett. 2003. Increased protein stability as a mechanism that enhances Nrf2-mediated transcriptional activation of the antioxidant response element. Degradation of Nrf2 by the 26 S proteasome. J Biol Chem 278: 4536-4541.

10. Cullinan, S. B., J. D. Gordan, J. Jin, J. W. Harper, and J. A. Diehl. 2004. The Keap1-BTB protein is an adaptor that bridges Nrf2 to a Cul3-based E3 ligase: oxidative stress sensing by a Cul3-Keap1 ligase. Mol Cell Biol 24: 8477-8486.

11. Kobayashi, A., M. I. Kang, H. Okawa, M. Ohtsuji, Y. Zenke, T. Chiba, K. Igarashi, and M. Yamamoto. 2004. Oxidative stress sensor Keap1 functions as an adaptor for Cul3-based E3 ligase to regulate proteasomal degradation of Nrf2. Mol Cell Biol 24: 7130-7139.

12. Zhang, D. D., S. C. Lo, J. V. Cross, D. J. Templeton, and M. Hannink. 2004. Keap1 is a redox-regulated substrate adaptor protein for a Cul3-dependent ubiquitin ligase complex. Mol Cell Biol 24: 10941-10953.

13. Dinkova-Kostova, A. T., W. D. Holtzclaw, R. N. Cole, K. Itoh, N. Wakabayashi, Y. Katoh, M. Yamamoto, and P. Talalay. 2002. Direct evidence that sulfhydryl groups of Keap1 are the sensors regulating induction of phase 2 enzymes that 
protect against carcinogens and oxidants. Proc Natl Acad Sci U S A 99: 1190811913.

14. Zhang, D. D., and M. Hannink. 2003. Distinct cysteine residues in Keap1 are required for Keap1-dependent ubiquitination of Nrf2 and for stabilization of Nrf2 by chemopreventive agents and oxidative stress. Mol Cell Biol 23: 8137-8151.

15. McMahon, M., D. J. Lamont, K. A. Beattie, and J. D. Hayes. 2010. Keap1 perceives stress via three sensors for the endogenous signaling molecules nitric oxide, zinc, and alkenals. Proc Natl Acad Sci U S A 107: 18838-18843.

16. Bloom, D. A., and A. K. Jaiswal. 2003. Phosphorylation of Nrf2 at Ser40 by protein kinase $\mathrm{C}$ in response to antioxidants leads to the release of $\mathrm{Nrf2}$ from INrf2, but is not required for Nrf2 stabilization/accumulation in the nucleus and transcriptional activation of antioxidant response element-mediated NAD(P)H:quinone oxidoreductase-1 gene expression. J Biol Chem 278: 4467544682.

17. Buckley, B. J., Z. M. Marshall, and A. R. Whorton. 2003. Nitric oxide stimulates Nrf2 nuclear translocation in vascular endothelium. Biochem Biophys Res Commun 307: 973-979.

18. Cullinan, S. B., D. Zhang, M. Hannink, E. Arvisais, R. J. Kaufman, and J. A. Diehl. 2003. Nrf2 is a direct PERK substrate and effector of PERK-dependent cell survival. Mol Cell Biol 23: 7198-7209.

19. Huang, H. C., T. Nguyen, and C. B. Pickett. 2002. Phosphorylation of Nrf2 at Ser-40 by protein kinase $\mathrm{C}$ regulates antioxidant response element-mediated transcription. J Biol Chem 277: 42769-42774. 
20. Yu, R., W. Lei, S. Mandlekar, M. J. Weber, C. J. Der, J. Wu, and A. N. Kong. 1999. Role of a mitogen-activated protein kinase pathway in the induction of phase II detoxifying enzymes by chemicals. J Biol Chem 274: 27545-27552.

21. Zipper, L. M., and R. T. Mulcahy. 2000. Inhibition of ERK and p38 MAP kinases inhibits binding of Nrf2 and induction of GCS genes. Biochem Biophys Res Commun 278: 484-492.

22. Itoh, K., T. Chiba, S. Takahashi, T. Ishii, K. Igarashi, Y. Katoh, T. Oyake, N. Hayashi, K. Satoh, I. Hatayama, M. Yamamoto, and Y. Nabeshima. 1997. An $\mathrm{Nrf} 2 / \mathrm{small}$ Maf heterodimer mediates the induction of phase II detoxifying enzyme genes through antioxidant response elements. Biochem Biophys Res Commun 236: 313-322.

23. Kwak, M. K., J. M. Cho, B. Huang, S. Shin, and T. W. Kensler. 2007. Role of increased expression of the proteasome in the protective effects of sulforaphane against hydrogen peroxide-mediated cytotoxicity in murine neuroblastoma cells. Free Radic Biol Med 43: 809-817.

24. Alam, J., D. Stewart, C. Touchard, S. Boinapally, A. M. Choi, and J. L. Cook. 1999. Nrf2, a Cap'n'Collar transcription factor, regulates induction of the heme oxygenase-1 gene. J Biol Chem 274: 26071-26078.

25. Nguyen, T., H. C. Huang, and C. B. Pickett. 2000. Transcriptional regulation of the antioxidant response element. Activation by Nrf2 and repression by MafK. $J$ Biol Chem 275: 15466-15473. 
26. Wild, A. C., H. R. Moinova, and R. T. Mulcahy. 1999. Regulation of gammaglutamylcysteine synthetase subunit gene expression by the transcription factor Nrf2. J Biol Chem 274: 33627-33636.

27. Venugopal, R., and A. K. Jaiswal. 1998. Nrf2 and Nrf1 in association with Jun proteins regulate antioxidant response element-mediated expression and coordinated induction of genes encoding detoxifying enzymes. Oncogene 17: 3145-3156.

28. Wasserman, W. W., and W. E. Fahl. 1997. Functional antioxidant responsive elements. Proc Natl Acad Sci U S A 94: 5361-5366.

29. Prestera, T., W. D. Holtzclaw, Y. Zhang, and P. Talalay. 1993. Chemical and molecular regulation of enzymes that detoxify carcinogens. Proc Natl Acad Sci U S A 90: 2965-2969.

30. Li, Y., and A. K. Jaiswal. 1992. Regulation of human NAD(P)H:quinone oxidoreductase gene. Role of AP1 binding site contained within human antioxidant response element. J Biol Chem 267: 15097-15104.

31. Gabrilovich, D. I., V. Bronte, S. H. Chen, M. P. Colombo, A. Ochoa, S. OstrandRosenberg, and H. Schreiber. 2007. The terminology issue for myeloid-derived suppressor cells. Cancer Res 67: 425; author reply 426.

32. Hanson, E. M., V. K. Clements, P. Sinha, D. Ilkovitch, and S. Ostrand-Rosenberg. 2009. Myeloid-derived suppressor cells down-regulate L-selectin expression on CD4+ and CD8+ T cells. J Immunol 183: 937-944. 
33. Liu, C., S. Yu, J. Kappes, J. Wang, W. E. Grizzle, K. R. Zinn, and H. G. Zhang. 2007. Expansion of spleen myeloid suppressor cells represses NK cell cytotoxicity in tumor-bearing host. Blood 109: 4336-4342.

34. Huang, B., P. Y. Pan, Q. Li, A. I. Sato, D. E. Levy, J. Bromberg, C. M. Divino, and S. H. Chen. 2006. Gr-1+CD115+ immature myeloid suppressor cells mediate the development of tumor-induced T regulatory cells and T-cell anergy in tumorbearing host. Cancer Res 66: 1123-1131.

35. Sinha, P., V. K. Clements, S. K. Bunt, S. M. Albelda, and S. Ostrand-Rosenberg. 2007. Cross-talk between myeloid-derived suppressor cells and macrophages subverts tumor immunity toward a type 2 response. J Immunol 179: 977-983.

36. Corzo, C. A., M. J. Cotter, P. Cheng, F. Cheng, S. Kusmartsev, E. Sotomayor, T. Padhya, T. V. McCaffrey, J. C. McCaffrey, and D. I. Gabrilovich. 2009.

Mechanism regulating reactive oxygen species in tumor-induced myeloid-derived suppressor cells. J Immunol 182: 5693-5701.

37. Ezernitchi, A. V., I. Vaknin, L. Cohen-Daniel, O. Levy, E. Manaster, A. Halabi, E. Pikarsky, L. Shapira, and M. Baniyash. 2006. TCR zeta down-regulation under chronic inflammation is mediated by myeloid suppressor cells differentially distributed between various lymphatic organs. J Immunol 177: 4763-4772.

38. Schmielau, J., and O. J. Finn. 2001. Activated granulocytes and granulocytederived hydrogen peroxide are the underlying mechanism of suppression of t-cell function in advanced cancer patients. Cancer Res 61: 4756-4760. 
39. Nagaraj, S., K. Gupta, V. Pisarev, L. Kinarsky, S. Sherman, L. Kang, D. L. Herber, J. Schneck, and D. I. Gabrilovich. 2007. Altered recognition of antigen is a mechanism of CD8+ T cell tolerance in cancer. Nat Med 13: 828-835.

40. Lu, T., R. Ramakrishnan, S. Altiok, J. I. Youn, P. Cheng, E. Celis, V. Pisarev, S. Sherman, M. B. Sporn, and D. Gabrilovich. 2011. Tumor-infiltrating myeloid cells induce tumor cell resistance to cytotoxic T cells in mice. J Clin Invest 121: 4015-4029.

41. Sinha, P., K. H. Parker, L. Horn, and S. Ostrand-Rosenberg. 2012. Tumorinduced myeloid-derived suppressor cell function is independent of IFN- $\gamma$ and IL4Ra. Eur J Immunol 42: 2052-2059.

42. Bunt, S. K., P. Sinha, V. K. Clements, J. Leips, and S. Ostrand-Rosenberg. 2006. Inflammation induces myeloid-derived suppressor cells that facilitate tumor progression. J Immunol 176: 284-290.

43. Chornoguz, O., L. Grmai, P. Sinha, K. A. Artemenko, R. A. Zubarev, and S. Ostrand-Rosenberg. 2011. Proteomic pathway analysis reveals inflammation increases myeloid-derived suppressor cell resistance to apoptosis. Mol Cell Proteomics 10: M110 002980.

44. Sinha, P., V. K. Clements, and S. Ostrand-Rosenberg. 2005. Reduction of myeloid-derived suppressor cells and induction of M1 macrophages facilitate the rejection of established metastatic disease. J Immunol 174: 636-645.

45. Parker, K., P. Sinha, L. Horn, V. Clements, and S. Ostrand-Rosenberg. 2014. HMGB1 enhances immune suppression by facilitating the differentiation and suppressive activity of myeloid-derived suppressor cells. Cancer Res. 
46. Sinha, P., and S. Ostrand-Rosenberg. 2013. Myeloid-derived suppressor cell function is reduced by Withaferin $\mathrm{A}$, a potent and abundant component of Withania somnifera root extract. Cancer Immunol Immunother 62: 1663-1673.

47. Beury, D. W., K. H. Parker, M. Nyandjo, P. Sinha, K. A. Carter, and S. OstrandRosenberg. 2014. Cross-talk among myeloid-derived suppressor cells, macrophages, and tumor cells impacts the inflammatory milieu of solid tumors. $J$ Leukoc Biol 96: 1109-1118.

48. Inoue, D., T. Suzuki, Y. Mitsuishi, Y. Miki, S. Suzuki, S. Sugawara, M. Watanabe, A. Sakurada, C. Endo, A. Uruno, H. Sasano, T. Nakagawa, K. Satoh, N. Tanaka, H. Kubo, H. Motohashi, and M. Yamamoto. 2012. Accumulation of p62/SQSTM1 is associated with poor prognosis in patients with lung adenocarcinoma. Cancer Sci 103: 760-766.

49. Shibata, T., A. Kokubu, M. Gotoh, H. Ojima, T. Ohta, M. Yamamoto, and S. Hirohashi. 2008. Genetic alteration of Keap1 confers constitutive Nrf2 activation and resistance to chemotherapy in gallbladder cancer. Gastroenterology 135: 1358-1368, 1368 e1351-1354.

50. Shibata, T., T. Ohta, K. I. Tong, A. Kokubu, R. Odogawa, K. Tsuta, H. Asamura, M. Yamamoto, and S. Hirohashi. 2008. Cancer related mutations in NRF2 impair its recognition by Keap1-Cul3 E3 ligase and promote malignancy. Proc Natl Acad Sci U S A 105: 13568-13573.

51. Singh, A., S. Boldin-Adamsky, R. K. Thimmulappa, S. K. Rath, H. Ashush, J. Coulter, A. Blackford, S. N. Goodman, F. Bunz, W. H. Watson, E. Gabrielson, E. Feinstein, and S. Biswal. 2008. RNAi-mediated silencing of nuclear factor 
erythroid-2-related factor 2 gene expression in non-small cell lung cancer inhibits tumor growth and increases efficacy of chemotherapy. Cancer Res 68: 79757984.

52. Solis, L. M., C. Behrens, W. Dong, M. Suraokar, N. C. Ozburn, C. A. Moran, A. H. Corvalan, S. Biswal, S. G. Swisher, B. N. Bekele, J. D. Minna, D. J. Stewart, and Wistuba, II. 2010. Nrf2 and Keap1 abnormalities in non-small cell lung carcinoma and association with clinicopathologic features. Clin Cancer Res 16: 3743-3753.

53. Yamamoto, T., K. Yoh, A. Kobayashi, Y. Ishii, S. Kure, A. Koyama, T. Sakamoto, K. Sekizawa, H. Motohashi, and M. Yamamoto. 2004. Identification of polymorphisms in the promoter region of the human NRF2 gene. Biochem Biophys Res Commun 321: 72-79.

54. Fahey, J. W., X. Haristoy, P. M. Dolan, T. W. Kensler, I. Scholtus, K. K. Stephenson, P. Talalay, and A. Lozniewski. 2002. Sulforaphane inhibits extracellular, intracellular, and antibiotic-resistant strains of Helicobacter pylori and prevents benzo[a]pyrene-induced stomach tumors. Proc Natl Acad Sci U S A 99: 7610-7615.

55. Khor, T. O., M. T. Huang, A. Prawan, Y. Liu, X. Hao, S. Yu, W. K. Cheung, J. Y. Chan, B. S. Reddy, C. S. Yang, and A. N. Kong. 2008. Increased susceptibility of Nrf2 knockout mice to colitis-associated colorectal cancer. Cancer Prev Res 1: 187-191.

56. Osburn, W. O., B. Karim, P. M. Dolan, G. Liu, M. Yamamoto, D. L. Huso, and T. W. Kensler. 2007. Increased colonic inflammatory injury and formation of 
aberrant crypt foci in Nrf2-deficient mice upon dextran sulfate treatment. Int $J$ Cancer 121: 1883-1891.

57. Suzuki, T., T. Shibata, K. Takaya, K. Shiraishi, T. Kohno, H. Kunitoh, K. Tsuta, K. Furuta, K. Goto, F. Hosoda, H. Sakamoto, H. Motohashi, and M. Yamamoto. 2013. Regulatory nexus of synthesis and degradation deciphers cellular Nrf2 expression levels. Mol Cell Biol 33: 2402-2412.

58. Xu, C., M. T. Huang, G. Shen, X. Yuan, W. Lin, T. O. Khor, A. H. Conney, and A. N. Kong. 2006. Inhibition of 7,12-dimethylbenz(a)anthracene-induced skin tumorigenesis in C57BL/6 mice by sulforaphane is mediated by nuclear factor E2-related factor 2. Cancer Res 66: 8293-8296.

59. Mazzoni, A., V. Bronte, A. Visintin, J. H. Spitzer, E. Apolloni, P. Serafini, P. Zanovello, and D. M. Segal. 2002. Myeloid suppressor lines inhibit T cell responses by an NO-dependent mechanism. J Immunol 168: 689-695.

60. Rodriguez, P. C., D. G. Quiceno, J. Zabaleta, B. Ortiz, A. H. Zea, M. B. Piazuelo, A. Delgado, P. Correa, J. Brayer, E. M. Sotomayor, S. Antonia, J. B. Ochoa, and A. C. Ochoa. 2004. Arginase I production in the tumor microenvironment by mature myeloid cells inhibits T-cell receptor expression and antigen-specific Tcell responses. Cancer Res 64: 5839-5849.

61. Srivastava, M. K., P. Sinha, V. K. Clements, P. Rodriguez, and S. OstrandRosenberg. 2010. Myeloid-derived suppressor cells inhibit T-cell activation by depleting cystine and cysteine. Cancer Res 70: 68-77. 
62. Bunt, S. K., V. K. Clements, E. M. Hanson, P. Sinha, and S. Ostrand-Rosenberg. 2009. Inflammation enhances myeloid-derived suppressor cell cross-talk by signaling through Toll-like receptor 4. J Leukoc Biol 85: 996-1004.

63. Gabrilovich, D. I., S. Ostrand-Rosenberg, and V. Bronte. 2012. Coordinated regulation of myeloid cells by tumours. Nat Rev Immunol 12: 253-268.

64. Lesokhin, A. M., T. M. Hohl, S. Kitano, C. Cortez, D. Hirschhorn-Cymerman, F. Avogadri, G. A. Rizzuto, J. J. Lazarus, E. G. Pamer, A. N. Houghton, T. Merghoub, and J. D. Wolchok. 2012. Monocytic CCR2(+) myeloid-derived suppressor cells promote immune escape by limiting activated CD8 T-cell infiltration into the tumor microenvironment. Cancer Res 72: 876-886.

65. Tsai, J. J., J. A. Dudakov, K. Takahashi, J. H. Shieh, E. Velardi, A. M. Holland, N. V. Singer, M. L. West, O. M. Smith, L. F. Young, Y. Shono, A. Ghosh, A. M. Hanash, H. T. Tran, M. A. Moore, and M. R. van den Brink. 2013. Nrf2 regulates haematopoietic stem cell function. Nat Cell Biol 15: 309-316.

66. Condamine, T., V. Kumar, I. R. Ramachandran, J. I. Youn, E. Celis, N. Finnberg, W. S. El-Deiry, R. Winograd, R. H. Vonderheide, N. R. English, S. C. Knight, H. Yagita, J. C. McCaffrey, S. Antonia, N. Hockstein, R. Witt, G. Masters, T. Bauer, and D. I. Gabrilovich. 2014. ER stress regulates myeloid-derived suppressor cell fate through TRAIL-R-mediated apoptosis. J Clin Invest 124: 2626-2639.

67. Ostrand-Rosenberg, S., and P. Sinha. 2009. Myeloid-derived suppressor cells: linking inflammation and cancer. J Immunol 182: 4499-4506. 
68. Parker, K. H., D. W. Beury, and S. Ostrand-Rosenberg. 2015. Myeloid-Derived Suppressor Cells: Critical Cells Driving Immune Suppression in the Tumor Microenvironment. Adv Cancer Res 128: 95-139.

69. Zoglmeier, C., H. Bauer, D. Norenberg, G. Wedekind, P. Bittner, N. Sandholzer, M. Rapp, D. Anz, S. Endres, and C. Bourquin. 2011. CpG blocks immunosuppression by myeloid-derived suppressor cells in tumor-bearing mice. Clin Cancer Res 17: 1765-1775.

70. Iclozan, C., S. Antonia, A. Chiappori, D. T. Chen, and D. Gabrilovich. 2013. Therapeutic regulation of myeloid-derived suppressor cells and immune response to cancer vaccine in patients with extensive stage small cell lung cancer. Cancer Immunol Immunother 62: 909-918.

71. Cheng, P., V. Kumar, H. Liu, J. I. Youn, M. Fishman, S. Sherman, and D. Gabrilovich. 2014. Effects of notch signaling on regulation of myeloid cell differentiation in cancer. Cancer Res 74: 141-152.

72. Wiers, K. M., D. M. Lathers, M. A. Wright, and M. R. Young. 2000. Vitamin D3 treatment to diminish the levels of immune suppressive CD34+ cells increases the effectiveness of adoptive immunotherapy. J Immunother 23: 115-124.

73. Wang, X. J., J. D. Hayes, C. J. Henderson, and C. R. Wolf. 2007. Identification of retinoic acid as an inhibitor of transcription factor Nrf2 through activation of retinoic acid receptor alpha. Proc Natl Acad Sci U S A 104: 19589-19594.

74. Al-Huseini, L. M., H. X. Aw Yeang, S. Sethu, N. Alhumeed, J. M. Hamdam, Y. Tingle, L. Djouhri, N. Kitteringham, B. K. Park, C. E. Goldring, and J. G. Sathish. 2013. Nuclear factor-erythroid 2 (NF-E2) p45-related factor-2 (Nrf2) modulates 
dendritic cell immune function through regulation of p38 MAPK-cAMPresponsive element binding protein/activating transcription factor 1 signaling. $J$ Biol Chem 288: 22281-22288.

75. Jaramillo, M. C., and D. D. Zhang. 2013. The emerging role of the Nrf2-Keap1 signaling pathway in cancer. Genes Dev 27: 2179-2191. 


\section{Chapter 4: Discussion}

\section{Summary of the major findings}

The research in this dissertation was focused on MDSC function and survival. Chapter 2 assessed the ability of MDSC to regulate the inflammatory milieu of solid tumors by participating in cross-talk with macrophages and tumor cells. MDSC and macrophages both have a dominant presence in solid tumors where they are major drivers of immune suppression and tumor progression (1). Previously, we had reported that cross-talk between these cells results in increased MDSC production of IL-10 and decreased macrophage production of IL-12, thereby polarizing the immune system towards a pro-tumor type 2 environment $(2,3)$. Additional factors were likely to be impacted by cross-talk between MDSC and macrophages, as well as by interactions with tumor cells. Therefore, we investigated how tumor cells, macrophages, and MDSC interact with respect to IL-6, TNF $\alpha$, IL-10, and nitric oxide (NO) since those molecules are present in many solid tumors and play important roles in tumor progression (4-9). The data presented in chapter 2 established that interplay between MDSC, macrophages, and tumor cells differentially impacts the production of IL-6, TNF $\alpha$, IL-10, and NO, and suggests that inflammation within solid tumors is regulated by the number of MDSC and macrophages present with the tumor.

Chapter 3 assessed how MDSC are able to survive and function in the TME. Solid tumors are an oxidatively stressed microenvironment due to hypoxia, and the presence of reactive oxygen species (ROS), which are created as a byproduct of metabolism and/or secretion by tumor-infiltrating cells (10). Therefore, MDSC present in the TME must 
mitigate oxidative stress in order to survive (1). Additionally, MDSC secrete ROS to suppress $\mathrm{T}$ cell activation and proliferation which further exposes them to oxidative radicals. However, MDSC do not seem to be negatively impacted by oxidative stress and are still capable of inhibiting anti-tumor immunity. Therefore, we hypothesized that MDSC are resistant to oxidative stress because they are protected by $\mathrm{Nrf} 2$, a transcription factor that is activated by oxidative radicals and regulates the synthesis of gene products that mitigate oxidative stress (11). The data presented in chapter 3 established that Nrf2 regulates the generation, survival and suppressive potency of MDSC, and that a feedback homeostatic mechanism maintains a steady-state level of circulating MDSC in tumorbearing individuals.

\section{How does Nrf2 impact inflammation, tumor progression, and anti-tumor immunity?}

As described in chapter 1, inflammation partially contributes to tumor progression by enhancing MDSC suppressive potency and accumulation, which causes dysfunctional anti-tumor immunity (12). However, inflammation also induces other mechanisms in tumor and/or stromal cells that promote tumor progression including: (i) increased ROS production which causes DNA mutations that not only cause inflammation by themselves, but also contribute to genetic instability which promotes the evolution of tumor cells to a heightened malignant state; (ii) the production of bioactive factors that stimulate tumor cell proliferation and survival; (iii) the production of cytokines and chemokines that recruit immune cells to the tumor and induce immunosuppressive cell populations such as Tregs and M2 macrophages; (iv) the production of proangiogenic factors such as VEGF, which enhances tumor vascularization; and (v) the production of 
matrix metalloproteases which enhance tumor cell invasiveness, motility, and metastasis (13-16). Since Nrf2 is known to decrease inflammation in addition to its antioxidant properties, we focused on how Nrf2 impacts MDSC accumulation, survival, suppressive potency, and tumor progression.

Nrf2 has classically been described as having anti-inflammatory properties in multiple pathologies and models. In a carrageenan-induced pleurisy model, $\mathrm{Nrf}^{-/-}$mice have a greater persistence of inflammatory neutrophils and macrophages in the plural cavity compared to wild type mice (17). Similarly, Nrf2-deficiency enhances the number of lung-infiltrating lymphocytes, neutrophils, and macrophages, and increases IL-6 expression in the lungs in a hyperoxia-induced lung injury model (18). In a UVB-induced skin inflammation model, Nrf2-deficency also enhances macrophage inflammatory protein -2 (MIP-2) expression, which is a chemoattractant for neutrophils (19). In a neuroinflammation model, LPS-treated $\mathrm{Nrf}^{-/-}$mice display hypersensitivity to LPS and their hippocampi have increased microglial cells and expression of the inflammatory mediators IL-6 and TNF $\alpha$ (20). LPS mediates its inflammatory effects by signaling through TLR4 and the transcription factor NF- $\mathrm{BB}$. Nrf2 seems to be involved in regulating NF- $\kappa \mathrm{B}$ in a post-translational manner, since Nrf2-deficient cells have higher expression of $\mathrm{p} 65-\mathrm{NF}-\kappa \mathrm{B}$ protein, but similar levels of $\mathrm{p} 65-\mathrm{NF}-\kappa \mathrm{B}$ mRNA compared to Nrf2-competent cells (21). Additionally, studies using mice with a mutation in the adenomatous polyposis gene $\left(\mathrm{APC}^{\mathrm{Min} /+}\right)$, which spontaneously develop intestinal tumors, show that Nrf2 deficiency also increases inflammation and the number of spontaneous tumors (22). Together, it seems as if Nrf2 is distinctly anti-inflammatory and can potentially quell tumor-promoting inflammation. 
However, the role that Nrf2 plays in anti-tumor immunity is not well established, and is controversial. Earlier murine studies that utilized intravenous injections of B16 melanoma suggested that $\mathrm{Nrf}^{-/-}$mice were more susceptible to pulmonary metastasis and $\mathrm{Nrf}^{+/+}$mice had reduced levels of MDSC (23). However, the authors failed to account for the fact that the level of MDSC is positively correlated with tumor burden. Since the $\mathrm{Nrf}^{---}$mice had a higher tumor burden, one would expect that those mice would naturally have a higher level of MDSC. The authors also incorrectly concluded that the $\mathrm{Nrf}^{-/-}$mice were more susceptible to pulmonary metastasis. Since their tumor model involved intravenous injection of B16 melanoma, they were observing the ability of B16 to colonize the lungs, which is not indicative of all phases of metastasis because it ignores extravasation, a critical step in metastatic development. However, B16 did appear to have an increased propensity to colonize the lungs of $\mathrm{Nrf2}^{--}$mice (23), which probably occurred because Nrf2 deficiency increases pulmonary inflammation, which would create an environment that favors tumor progression (24-26). The authors also observed that $\mathrm{Nrf}^{-/-}$MDSC had more ROS, and suggested that $\mathrm{Nrf}^{-/-}$were more suppressive than $\mathrm{Nrf}^{+/+}$MDSC. However, they did not compare suppressive activity between $\mathrm{Nrf}^{+/+}$and $\mathrm{Nrf}^{-/-}$MDSC, so they prematurely and possibly incorrectly concluded that Nrf2 deficiency enhances MDSC suppressive potency. In contrast, the data presented in this dissertation clearly demonstrate that Nrf2 enhances MDSC-mediated suppression, and that Nrf2 enhances the capacity for MDSC to produce extracellular $\mathrm{H}_{2} \mathrm{O}_{2}$, which is a primary mechanism that MDSC utilize to facilitate suppression of $\mathrm{T}$ cell activation and proliferation which is further evidence that Nrf2 enhances MDSC activity. Additionally, the authors observed a higher level of MDSC in the tumors of $\mathrm{Nrf}^{-{ }^{--}}$mice, but did not 
account for similar tumor burden between $\mathrm{Nrf}^{+/+}$and $\mathrm{Nrf}^{-/-}$mice. Since it has been well established that the level of circulating MDSC increases with tumor burden, higher levels of MDSC in the tumor could be from the increased opportunity of MDSC to migrate to the tumor. Additionally, this dissertation demonstrates that Nrf2 increases the intratumoral presence of MDSC, indicating that there are a higher number of MDSC to elicit immune suppression in the tumor, which disagrees with their observation that Nrf2 deficiency promotes MDSC presence in the tumor. Therefore, it seems as if Nrf2 may play a protective role in tumor colonization of the lungs, however, Nrf 2 clearly enhances MDSC survival, suppressive activity, and presence in the tumor.

Nrf2 probably does not exclusively promote MDSC survival and suppressive activity, since both therapeutic activation and inhibition of Nrf2 have been shown to mitigate the suppressive activity of MDSC. For example, CDDO-Me (Methyl 2-cyano3,12-dioxooleana-1,9(11)dien-28-oate) is a synthetic triterpenoid that is a potent activator of Nrf2 (27). Treatment of tumor-bearing mice with CDDO-Me reduces intracellular ROS in MDSC which is correlated with a reduction in MDSC suppressive activity (28). In contrast, Withaferin A, a natural compound isolated form Withania somnifera, has been shown to bind to Nrf2 and inhibit its translocation to the nucleus (29). Consequently, treatment of tumor-bearing mice with Withaferin A reduces the amount of PMN-MDSC, decreases MDSC production of extracellular $\mathrm{H}_{2} \mathrm{O}_{2}$, and reduces their suppressive activity (30). Therefore, on the surface it seems as if there is an optimal level of Nrf2 activity that promotes MDSC suppressive activity. However, both CDDO-Me

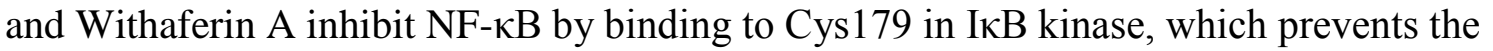

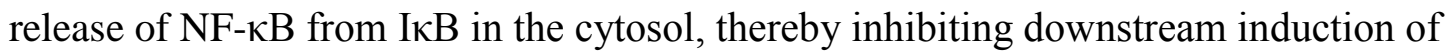


pro-inflammatory signaling $(31,32)$. Since inflammation is a driver of immune suppression and tumor progression, the therapeutic efficacy of these drugs is probably due to their anti-inflammatory effects, in addition to their regulation of Nrf2.

\section{How does MDSC production of IL-10 impact anti-tumor immunity?}

IL-10 is a pleotropic, anti-inflammatory cytokine that has both pro- and antitumor activity. IL-10 is produced by virtually all leukocytes, including macrophages, DC, NK cells, neutrophils, MDSC, eosinophils, mast cells, and most $\mathrm{CD}^{+}{ }^{+} \mathrm{T}$ cell subsets (including Th1, Th2, Th9, Th17, Th22, Treg and Tr1) (33, 34). IL-10 signals through the IL-10 receptor, which is an oligomeric membrane protein consisting of IL-10R1 and IL10R2. While most nucleated cells express IL-10R2, IL-10 signaling typically occurs only in immune cells since IL-10R1 is expression is primarily restricted to leukocytes (33). Binding of IL-10 to the IL-10 receptor activates two members of the Janus Kinase family: JAK1 (associated with IL-10R1) and TYK2 (associated with IL-10R2) (35). Binding of JAK1 and TYK2 to the IL-10 receptor facilitates the phosphorylation of two tyrosines (Tyr446 and Tyr496) of IL-10R1, which enables the transcription factor STAT3 to bind and in turn become phosphorylated and activated $(35,36)$. Additionally, STAT1 and STAT5 are known to be activated during IL-10 signaling $(35,37)$. These activated transcription factors then homo- and heterodimerize, translocate to the nucleus, bind STAT binding elements of various promoters, and induce transcription of the corresponding genes.

Stimulation of IL-10 receptor results in the reduction of inflammatory activity in the target cell by altering the synthesis of many genes. IL-10 inhibits the secretion of 
many pro-inflammatory cytokines such as IFN $\gamma$, TNF $\alpha$, IL-1 $\beta$, IL-2, IL-6, IL-8, IL-12, GCSF, and GM-CSF $(33,38,39)$. IL-10 also enhances the release of anti-inflammatory mediators such as IL-1 receptor antagonist and soluble TNF $\alpha$ receptors, which sequester IL-1 $\beta$ and TNF $\alpha$, respectively (40-42). IL-10 is able to exert some of its antiinflammatory effects by increasing the expression of several molecules that interfere with inflammatory signaling including: (i) Bcl3, which impairs the ability of NF- $\mathrm{kB}$ to bind DNA, and recruits HDAC1 which promotes epigenetic silencing of genes such as TNFa (43-45); (ii) Etv3, a transcriptional co-repressor that inhibits NF-kB activity (46) (iii) SHIP-1, which inhibits TNF $\alpha$ translation (47); (iv) decreases miR-155, which targets SHIP-1 (48); (v) Nfil3, which suppresses the expression of the IL-12 subunit p40 (49, 50); (vi) SBNO2, another transcriptional co-repressor that inhibits NF-kB (46); (vii) Zfp36, an RNA-binding protein that targets $\mathrm{TNF} \alpha(45,51,52)$ and; (viii) SOCS3, which binds Janus kinases and inhibits inflammatory signaling mediated by the JAK/STAT pathway (e.g. IL-6, IFN $\gamma$, GM-CSF) (53). Because IL-10 utilizes multiple mechanisms to inhibit inflammation, IL-10 can inhibit tumor progression by decreasing tumor-promoting inflammation.

IL-10 has been demonstrated to have multiple anti-tumor mechanisms in both human and mouse studies. Some of these anti-tumor mechanisms are attributed to the ability of IL-10 to reduce tumor-promoting inflammation. Evidence linking IL-10's role in suppressing tumor-promoting inflammation include the observations that IL- $10^{-/-}$mice spontaneously develop irritable bowel disease and colon cancer (54), and humans deficient in IL-10 signaling develop lymphomas at a young age (55). Furthermore, mice with a mutation in the adenomatous polyposis gene $\left(\mathrm{APC}^{\Delta 468}\right)$ show that T-cell-specific 
ablation of IL-10 dramatically changes the inflammatory milieu and enhances tumor burden (56). Additionally, a study using $\mathrm{APC}^{\mathrm{Min} /+}$ demonstrated that adoptive transfer of Tregs, which produce IL-10, reduce tumor burden in an IL-10-dependent manner (57). Additional studies that utilized IL- $10^{-/-}$mice show that IL-10 reduces tumorigenesis, tumor growth, and metastasis $(58,59)$.

IL-10 can also enhance anti-tumor immunity by increasing the activity of NK and tumor-reactive $\mathrm{CD}^{+} \mathrm{T}$ cells. IL-10 enhances NK activity against human melanoma cells by decreasing tumor cell expression of MHC I $(60,61)$. MHC I is responsible for presenting endogenous tumor antigens to $\mathrm{CD} 8^{+} \mathrm{T}$ cells. However, MHC I also serves as an NK inhibitory receptor. IL-10 indirectly decreases transporter associated with antigen processing (TAP) -1 and -2, which are responsible for transporting processed peptides from the cytosol to the lumen of the endoplasmic reticulum (ER). Peptides in the ER are loaded onto MHC I, which stabilizes and enables the MHC I complex to be presented on the cell surface. Therefore, IL-10 decreases MHC I expression by limiting the availability of peptides for MHC I complexes. Since tumor cell expression of MHC I blocks NK cellmediated killing of tumor cells, decreased tumor cell expression of MHC I will result in a higher level of NK cell activity.

Additionally, treatment of tumor-bearing mice with pegylated IL-10 (peg-IL-10), a form of IL-10 that has increased serum half-life, induces IFN $\gamma$ and granzyme-B production by tumor-infiltrating $\mathrm{CD}^{+} \mathrm{T}$ cells and reduces tumor burden in a $12-\mathrm{O}-$ tetradecanoyl-phorbol acetate (TPA)-induced skin carcinoma model, a transplantable PVD6 squamous cell carcinoma model, and a spontaneous mammary (MMTVneu) tumor model (62). IFN $\gamma$ enhances antigen presentation and $\mathrm{T}$ cell activation in a positive 
feedback manner and increases anti-tumor activity elicited by T cells. Granzyme-B is utilized by $\mathrm{CD} 8^{+} \mathrm{T}$ cells to elicit tumor cell killing. Therefore, treatment of mice with peg-IL-10 increases the activity of tumor-reactive $\mathrm{CD} 8^{+} \mathrm{T}$ cells. Peg-IL-10 has also shown some therapeutic efficacy as an adjuvant for immunotherapy in mice. Experiments utilizing mice bearing OVA-expressing PVD6 squamous cell carcinoma show that treatment with peg-IL-10 results in tumor rejection when combined with the adoptive transfer of OVA-specific CD8 ${ }^{+} \mathrm{T}$ cells (63). The therapeutic effects of peg-IL-10 on T cells could be caused by IL-10-mediated upregulation of SHIP-1 and the reduction of the SHIP-1 inhibitory microRNA miR-155 $(47,48)$. SHIP-1 activates the PI3 kinase pathway and is required for $\mathrm{T}$ cell activation and proliferation (64). Therefore, IL-10 can also promote anti-tumor immunity by enhancing $\mathrm{CD} 8^{+} \mathrm{T}$ cell activity.

However, IL-10 also has pro-tumor activity. Some of IL-10's pro-tumor activity has been attributed to the impairment of DC function. Studies utilizing S1509a fibrosarcoma-bearing mice show that IL-10 can impair tumor antigen presentation by DC, thereby potentially preventing $\mathrm{T}$ cells from mounting an effective anti-tumor immune response (65). IL-10 reduces DC antigen presentation through multiple mechanisms including: (i) decreasing MHC II expression, which is essential for tumor antigen presentation to $\mathrm{CD} 4^{+}$cells; (ii) decreasing intracellular adhesion molecules (e.g. ICAM-1) that are essential for the formation of the immunological synapse, which is a cell-to-cell signaling structure that forms during antigen presentation between APC and T cells; (iii) decreasing costimulatory molecules (e.g. CD80 and CD86), which are essential for T cell activation, and; (iv) decreasing Th1 cytokines (e.g. IL-12), which facilitate the activation of the immune system toward an anti-tumor response $(9,66,67)$. Indeed, in 
vitro studies utilizing human DC and T cells show that IL-10 treated DC induce a state of anergy of $\mathrm{CD}^{+}$and $\mathrm{CD} 8^{+} \mathrm{T}$ cells in non-tumor systems (68-70), and in melanomareactive $\mathrm{CD}^{+} \mathrm{T}$ cells (71), supporting the idea that IL-10-mediated impairment of DC activity facilitates tumor progression.

IL-10 also induces Tregs, which as previously discussed (chapter 1, section 2.5), can inhibit anti-tumor immunity (72), and studies using mice bearing MC38 colon carcinoma show that Tregs are a major source of IL-10 in the TME (73). IL-10 enhances Treg production of TGF $\beta$ in B16 melanoma-bearing mice, which results in systemic suppression of anti-tumor immunity (74). Additionally, IL-10 upregulates expression of the TGF $\beta$ receptor 2 , thereby enhancing the capacity for $\mathrm{T}$ cells to be negatively regulated by TGF $\beta$ (75). Therefore, IL-10 not only decreases anti-tumor immunity by inducing Tregs, but also enhances their immunosuppressive activity.

IL-10 can also favor tumor growth since IL-10 $0^{-/}$mice bearing 4T1 mammary carcinoma have slower growing tumors and live longer than tumor-bearing wild type mice (76). Additionally, 4T1-induced MDSC derived from IL-10 ${ }^{-/}$mice are less suppressive than 4T1-induced MDSC from wild type mice, indicating an increased propensity for IL-10-suffient MDSC to inhibit anti-tumor immunity and promote tumor progression (76). While IL-10 could be promoting tumor progression through MDSC and decreasing anti-tumor immunity, IL-10 could also be directly enhancing tumor growth since in vitro experiments indicate that IL-10 stimulates tumor cell proliferation and inhibits apoptosis $(77,78)$. Another direct effect of IL-10 on tumor cells includes the downregulation of MHC I expression in human melanoma cells, which decreases the cytolytic activity of tumor-reactive CD ${ }^{+}$cells $(60,61)$. Similarly, IL-10 production by 
human basal and squamous cell carcinomas prevents in vitro lysis of malignant cells by tumor-infiltrating lymphocytes (79). Furthermore, IL-10 expression by human melanoma cells is correlated with tumor progression and metastasis, and indicates that melanoma cell expression of IL-10 is an important prognostic indicator of disease progression (80). Together, these observations support the notion that IL-10 inhibits anti-tumor immunity and favors tumor growth.

MDSC are capable of producing IL-10, and cell-to-cell contact between MDSC and macrophages synergistically enhances MDSC IL-10 (3). However, the mechanism responsible for mediating this cell-to-cell enhancement of MDSC and macrophages is not known. Currently, it is known that TLR4 signaling is required for MDSC production of IL-10, since TLR4 ${ }^{-/-}$MDSC do not produce IL-10 (2). However, TLR4 expression is only required on MDSC and not macrophages since $\mathrm{TLR} 4^{-/-}$macrophages are equally capable as $\mathrm{TLR}^{+/+}$macrophages in enhancing MDSC IL-10 in a cell-to-cell contact dependent manner. Therefore, the ligand responsible for cell-to-cell interactions between MDSC and macrophages does not require TLR4.

Inflammation also enhances the ability for MDSC to produce IL-10 since MDSC induced by 4T1 tumors that were engineered to constitutively produce high levels of IL$1 \beta(4 \mathrm{~T} 1 / \mathrm{IL}-1 \beta)$ make more IL-10 than MDSC induced by parental 4T1 tumors (2). In contrast, some inflammatory mediators decrease MDSC IL-10, because MDSC derived from IL-6 ${ }^{-/-}$mice make substantially more IL-10 than MDSC derived from IL- $6^{+/+}$mice. Interestingly, IL-6 does not directly impact MDSC synthesis of IL-10 which suggests that MDSC derived from IL- $6^{-/-}$mice have epigenetic changes that result in the increased capacity for MDSC to synthesize IL-10 (76). This observation might occur because IL-6 
signaling activates STAT3, which mediates the synthesis of $\mathrm{Bcl} 3$. In turn, $\mathrm{Bcl} 3$ recruits HDAC1, which is involved in rearrangement of histones and epigenetic silencing (4345). Therefore, IL-6 could be inhibiting IL-10 in MDSC in a Bcl3- and HDAC1dependent manner.

Nrf2 also plays a role in modulating IL-10 production in MDSC since $\mathrm{Nrf}^{-/-}$ MDSC make more IL-10 than Nrf2 ${ }^{+/+}$MDSC. Nrf2 might be modulating IL-10 in MDSC though the p38-MAPK pathway, since $\mathrm{Nrf}^{-/-}$immature dendritic cells (iDC) display elevated levels of IL-10 due to heightened activity of the p38 MAPK pathway (81). p38, along with ERK, represent two major groups of kinases involved in the MAPK signaling cascade and are necessary for the maximum level production of IL-10 induced by TLR signaling (82). Interestingly, TNF $\alpha$ and IL-1, as well as TLR4 stimulation, also induce p38 MAPK activation, which is consistent with the idea that inflammation enhances IL10 production $(83)$.

\section{How does cross-talk impact other cells in the tumor microenvironment?}

This dissertation characterized how cross-talk between MDSC, macrophages, and tumor cells impacts the levels of IL-6, IL-10, IL-12, TNF $\alpha$, and NO. We also briefly examined MSDC and macrophage secretion of TGF $\beta 1$, TGF $\beta 2$, TGF $\beta 3$, GM-CSF, IL- $1 \beta$, CCL2, MIP-1 $\alpha$, IL-4, IL-13, IL-23, and VEGF, and how cross-talk between MDSC and macrophages impacts the secretion of those inflammatory mediators. The tumor microenvironment also contains other cell types such as T and B lymphocytes, NK cells, tumor-associated fibroblasts, neutrophils, eosinophils, mast cells, and dendritic cells which could potentially be affected by MDSC cross-talk and subsequently promote tumor 
progression (84-86). For example, mast cells participate in cross-talk with MDSC (8789). Activated mast cells release histamine, which increases the suppressive potency of M-MDSC by increasing MDSC expression of ARG1 and NOS2 (88). Mast cells are also induced to produce more histamine by IL-6 (90). Therefore, a complex relationship potentially exists between tumor cells, MDSC, and mast cells since cross-talk between tumor cells and MDSC increases IL-6 in the TME (76), and IL-6 enhances mast cell production of histamine which enhances MDSC suppressive potency (88). Because high levels of IL-6 in the serum are correlated with chronic inflammation, tumor burden, and poor prognosis in multiple tumor systems (91), and histamine and IL-6 enhance MDSC suppressive activity $(76,92-94)$, cross-talk between tumor cells, MDSC, and mast cells can promote tumor progression by altering inflammation in the TME and by further decreasing anti-tumor immunity.

Additionally, Nrf2 could play a role in this complex feedback loop since Nrf2 positively regulates IL-6 production (95), and oxidative stress enhances mast cell production of IL-6 (96). This dissertation also demonstrates that Nrf2 increases the amount of MDSC present in the tumor. Higher levels of MDSC afford more opportunities for MDSC to participate in cross-talk with tumor cells and mast cells, which would ultimately enhance the amount of IL- 6 and histamine produced in the TME. Therefore, Nrf2 impacts the inflammatory milieu of the TME by virtue of altering the level of MDSC present in the tumor.

Clearly there are a large amount of potential interactions and opportunities for cross-talk to occur in the TME. Since inflammation is a major driver of immune suppression and cancer progression, understanding how cells modulate inflammation in 
the TME is important for identifying therapeutic targets to reduce inflammation, immune suppression, and tumor progression.

\section{Concluding remarks}

The goal of this dissertation was to characterize how cross-talk between MDSC, macrophages, and tumor cells alters the inflammatory milieu of the TME which ultimately impacts tumor progression, and to characterize the role that Nrf2 plays in aiding MDSC suppressive activity and helping MDSC mitigate oxidative radicals and apoptosis. The findings presented in this dissertation provide a broader understanding of how MDSC are able to function, how they function, and survive in the TME. While this dissertation concludes that the level of MDSC present in the TME is likely to have profound effects on the inflammatory milieu, other cells present in the tumor are likely to be impacting the inflammatory milieu and altering the balance between pro- and antitumor immunity. Similarly, Nrf2 may be impacting other cell types involved in pro- and anti-tumor immunity. MDSC remain a major obstacle for effective cancer immunotherapies and targeting Nrf2 in MDSC to alter inflammation in the TME and enhance anti-tumor immunity should be explored as a potential treatment option. 


\section{References}

1. Gabrilovich, D. I., S. Ostrand-Rosenberg, and V. Bronte. 2012. Coordinated regulation of myeloid cells by tumours. Nat Rev Immunol 12: 253-268.

2. Bunt, S. K., V. K. Clements, E. M. Hanson, P. Sinha, and S. Ostrand-Rosenberg. 2009. Inflammation enhances myeloid-derived suppressor cell cross-talk by signaling through Toll-like receptor 4. J Leukoc Biol 85: 996-1004.

3. Sinha, P., V. K. Clements, S. K. Bunt, S. M. Albelda, and S. Ostrand-Rosenberg. 2007. Cross-talk between myeloid-derived suppressor cells and macrophages subverts tumor immunity toward a type 2 response. J Immunol 179: 977-983.

4. Becker, C., M. C. Fantini, S. Wirtz, A. Nikolaev, H. A. Lehr, P. R. Galle, S. RoseJohn, and M. F. Neurath. 2005. IL-6 signaling promotes tumor growth in colorectal cancer. Cell Cycle 4: 217-220.

5. Su, Y. W., T. X. Xie, D. Sano, and J. N. Myers. 2011. IL-6 stabilizes Twist and enhances tumor cell motility in head and neck cancer cells through activation of casein kinase 2. PLoS One 6: e19412.

6. Santer, F. R., K. Malinowska, Z. Culig, and I. T. Cavarretta. 2010. Interleukin-6 trans-signalling differentially regulates proliferation, migration, adhesion and maspin expression in human prostate cancer cells. Endocr Relat Cancer 17: 241253.

7. Balkwill, F. 2006. TNF-alpha in promotion and progression of cancer. Cancer Metastasis Rev 25: 409-416. 
8. Bronte, V., and P. Zanovello. 2005. Regulation of immune responses by Larginine metabolism. Nat Rev Immunol 5: 641-654.

9. Mocellin, S., F. M. Marincola, and H. A. Young. 2005. Interleukin-10 and the immune response against cancer: a counterpoint. J Leukoc Biol 78: 1043-1051.

10. Liou, G. Y., and P. Storz. 2010. Reactive oxygen species in cancer. Free Radic Res 44: 479-496.

11. Ma, Q. 2013. Role of nrf2 in oxidative stress and toxicity. Annu Rev Pharmacol Toxicol 53: 401-426.

12. Parker, K. H., D. W. Beury, and S. Ostrand-Rosenberg. 2015. Myeloid-Derived Suppressor Cells: Critical Cells Driving Immune Suppression in the Tumor Microenvironment. Adv Cancer Res 128: 95-139.

13. DeNardo, D. G., P. Andreu, and L. M. Coussens. 2010. Interactions between lymphocytes and myeloid cells regulate pro- versus anti-tumor immunity. Cancer Metastasis Rev 29: 309-316.

14. Grivennikov, S. I., F. R. Greten, and M. Karin. 2010. Immunity, inflammation, and cancer. Cell 140: 883-899.

15. Ostrand-Rosenberg, S., and P. Sinha. 2009. Myeloid-derived suppressor cells: linking inflammation and cancer. J Immunol 182: 4499-4506.

16. Qian, B. Z., and J. W. Pollard. 2010. Macrophage diversity enhances tumor progression and metastasis. Cell 141: 39-51.

17. Itoh, K., M. Mochizuki, Y. Ishii, T. Ishii, T. Shibata, Y. Kawamoto, V. Kelly, K. Sekizawa, K. Uchida, and M. Yamamoto. 2004. Transcription factor Nrf2 
regulates inflammation by mediating the effect of 15-deoxy-Delta(12,14)prostaglandin j(2). Molecular and cellular biology 24: 36-45.

18. Reddy, N. M., S. R. Kleeberger, T. W. Kensler, M. Yamamoto, P. M. Hassoun, and S. P. Reddy. 2009. Disruption of Nrf2 impairs the resolution of hyperoxiainduced acute lung injury and inflammation in mice. Journal of Immunology 182: 7264-7271.

19. Saw, C. L., A. Y. Yang, M. T. Huang, Y. Liu, J. H. Lee, T. O. Khor, Z. Y. Su, L. Shu, Y. Lu, A. H. Conney, and A. N. Kong. 2014. Nrf2 null enhances UVBinduced skin inflammation and extracellular matrix damages. Cell \& bioscience 4: 39.

20. Innamorato, N. G., A. I. Rojo, A. J. Garcia-Yague, M. Yamamoto, M. L. de Ceballos, and A. Cuadrado. 2008. The transcription factor Nrf2 is a therapeutic target against brain inflammation. Journal of Immunology 181: 680-689.

21. Cuadrado, A., Z. Martin-Moldes, J. Ye, and I. Lastres-Becker. 2014. Transcription factors NRF2 and NF-kappaB are coordinated effectors of the Rho family, GTP-binding protein RAC1 during inflammation. J Biol Chem 289: $15244-15258$.

22. Cheung, K. L., J. H. Lee, T. O. Khor, T. Y. Wu, G. X. Li, J. Chan, C. S. Yang, and A. N. Kong. 2014. Nrf2 knockout enhances intestinal tumorigenesis in $\operatorname{Apc}(\mathrm{min} /+)$ mice due to attenuation of anti-oxidative stress pathway while potentiates inflammation. Molecular carcinogenesis 53: 77-84.

23. Satoh, H., T. Moriguchi, K. Taguchi, J. Takai, J. M. Maher, T. Suzuki, P. T. Winnard, Jr., V. Raman, M. Ebina, T. Nukiwa, and M. Yamamoto. 2010. Nrf2- 
deficiency creates a responsive microenvironment for metastasis to the lung. Carcinogenesis 31: 1833-1843.

24. Jin, W., L. Zhu, Q. Guan, G. Chen, Q. F. Wang, H. X. Yin, C. H. Hang, J. X. Shi, and H. D. Wang. 2008. Influence of Nrf2 genotype on pulmonary NF-kappaB activity and inflammatory response after traumatic brain injury. Ann Clin Lab Sci 38: $221-227$.

25. Ishii, Y., K. Itoh, Y. Morishima, T. Kimura, T. Kiwamoto, T. Iizuka, A. E. Hegab, T. Hosoya, A. Nomura, T. Sakamoto, M. Yamamoto, and K. Sekizawa. 2005. Transcription factor Nrf2 plays a pivotal role in protection against elastaseinduced pulmonary inflammation and emphysema. Journal of Immunology 175: 6968-6975.

26. Jin, W., H. Wang, Y. Ji, L. Zhu, W. Yan, L. Qiao, and H. Yin. 2009. Genetic ablation of Nrf2 enhances susceptibility to acute lung injury after traumatic brain injury in mice. Exp Biol Med (Maywood) 234: 181-189.

27. Thimmulappa, R. K., R. J. Fuchs, D. Malhotra, C. Scollick, K. Traore, J. H. Bream, M. A. Trush, K. T. Liby, M. B. Sporn, T. W. Kensler, and S. Biswal. 2007. Preclinical evaluation of targeting the Nrf2 pathway by triterpenoids (CDDO-Im and CDDO-Me) for protection from LPS-induced inflammatory response and reactive oxygen species in human peripheral blood mononuclear cells and neutrophils. Antioxidants \& redox signaling 9: 1963-1970.

28. Nagaraj, S., J. I. Youn, H. Weber, C. Iclozan, L. Lu, M. J. Cotter, C. Meyer, C. R. Becerra, M. Fishman, S. Antonia, M. B. Sporn, K. T. Liby, B. Rawal, J. H. Lee, and D. I. Gabrilovich. 2010. Anti-inflammatory triterpenoid blocks immune 
suppressive function of MDSCs and improves immune response in cancer. Clin Cancer Res 16: 1812-1823.

29. Vaishnavi, K., N. Saxena, N. Shah, R. Singh, K. Manjunath, M. Uthayakumar, S. P. Kanaujia, S. C. Kaul, K. Sekar, and R. Wadhwa. 2012. Differential activities of the two closely related withanolides, Withaferin A and Withanone: bioinformatics and experimental evidences. PLoS One 7: e44419.

30. Sinha, P., and S. Ostrand-Rosenberg. 2013. Myeloid-derived suppressor cell function is reduced by Withaferin A, a potent and abundant component of Withania somnifera root extract. Cancer immunology, immunotherapy : CII 62: 1663-1673.

31. Heyninck, K., M. Lahtela-Kakkonen, P. Van der Veken, G. Haegeman, and W. Vanden Berghe. 2014. Withaferin A inhibits NF-kappaB activation by targeting cysteine 179 in IKKbeta. Biochem Pharmacol 91: 501-509.

32. Ahmad, R., D. Raina, C. Meyer, S. Kharbanda, and D. Kufe. 2006. Triterpenoid CDDO-Me blocks the NF-kappaB pathway by direct inhibition of IKKbeta on Cys-179. J Biol Chem 281: 35764-35769.

33. Sabat, R., G. Grutz, K. Warszawska, S. Kirsch, E. Witte, K. Wolk, and J. Geginat. 2010. Biology of interleukin-10. Cytokine Growth Factor Rev 21: 331-344.

34. Tan, C., and I. Gery. 2012. The unique features of Th9 cells and their products. Critical reviews in immunology 32: 1-10.

35. Finbloom, D. S., and K. D. Winestock. 1995. IL-10 induces the tyrosine phosphorylation of tyk2 and Jak1 and the differential assembly of STAT1 alpha 
and STAT3 complexes in human T cells and monocytes. Journal of Immunology 155: 1079-1090.

36. Weber-Nordt, R. M., J. K. Riley, A. C. Greenlund, K. W. Moore, J. E. Darnell, and R. D. Schreiber. 1996. Stat3 recruitment by two distinct ligand-induced, tyrosine-phosphorylated docking sites in the interleukin-10 receptor intracellular domain. J Biol Chem 271: 27954-27961.

37. Wehinger, J., F. Gouilleux, B. Groner, J. Finke, R. Mertelsmann, and R. M. Weber-Nordt. 1996. IL-10 induces DNA binding activity of three STAT proteins (Stat1, Stat3, and Stat5) and their distinct combinatorial assembly in the promoters of selected genes. FEBS letters 394: 365-370.

38. de Waal Malefyt, R., J. Abrams, B. Bennett, C. G. Figdor, and J. E. de Vries. 1991. Interleukin 10(IL-10) inhibits cytokine synthesis by human monocytes: an autoregulatory role of IL-10 produced by monocytes. The Journal of experimental medicine 174: 1209-1220.

39. Fiorentino, D. F., A. Zlotnik, T. R. Mosmann, M. Howard, and A. O'Garra. 1991. IL-10 inhibits cytokine production by activated macrophages. Journal of Immunology 147: 3815-3822.

40. Hart, P. H., E. K. Hunt, C. S. Bonder, C. J. Watson, and J. J. Finlay-Jones. 1996. Regulation of surface and soluble TNF receptor expression on human monocytes and synovial fluid macrophages by IL-4 and IL-10. Journal of Immunology 157: $3672-3680$. 
41. Jenkins, J. K., M. Malyak, and W. P. Arend. 1994. The effects of interleukin-10 on interleukin-1 receptor antagonist and interleukin-1 beta production in human monocytes and neutrophils. Lymphokine Cytokine Res 13: 47-54.

42. Joyce, D. A., D. P. Gibbons, P. Green, J. H. Steer, M. Feldmann, and F. M. Brennan. 1994. Two inhibitors of pro-inflammatory cytokine release, interleukin10 and interleukin-4, have contrasting effects on release of soluble p75 tumor necrosis factor receptor by cultured monocytes. European journal of immunology 24: $2699-2705$.

43. Kuwata, H., Y. Watanabe, H. Miyoshi, M. Yamamoto, T. Kaisho, K. Takeda, and S. Akira. 2003. IL-10-inducible Bcl-3 negatively regulates LPS-induced TNFalpha production in macrophages. Blood 102: 4123-4129.

44. Wessells, J., M. Baer, H. A. Young, E. Claudio, K. Brown, U. Siebenlist, and P. F. Johnson. 2004. BCL-3 and NF-kappaB p50 attenuate lipopolysaccharideinduced inflammatory responses in macrophages. J Biol Chem 279: 49995-50003.

45. Hutchins, A. P., D. Diez, Y. Takahashi, S. Ahmad, R. Jauch, M. L. Tremblay, and D. Miranda-Saavedra. 2013. Distinct transcriptional regulatory modules underlie STAT3's cell type-independent and cell type-specific functions. Nucleic acids research 41: 2155-2170.

46. El Kasmi, K. C., A. M. Smith, L. Williams, G. Neale, A. D. Panopoulos, S. S. Watowich, H. Hacker, B. M. Foxwell, and P. J. Murray. 2007. Cutting edge: A transcriptional repressor and corepressor induced by the STAT3-regulated antiinflammatory signaling pathway. Journal of Immunology 179: 7215-7219. 
47. Chan, C. S., A. Ming-Lum, G. B. Golds, S. J. Lee, R. J. Anderson, and A. L. Mui. 2012. Interleukin-10 inhibits lipopolysaccharide-induced tumor necrosis factoralpha translation through a SHIP1-dependent pathway. J Biol Chem 287: 3802038027.

48. McCoy, C. E., F. J. Sheedy, J. E. Qualls, S. L. Doyle, S. R. Quinn, P. J. Murray, and L. A. O'Neill. 2010. IL-10 inhibits miR-155 induction by toll-like receptors. $J$ Biol Chem 285: 20492-20498.

49. Kobayashi, T., K. Matsuoka, S. Z. Sheikh, H. Z. Elloumi, N. Kamada, T. Hisamatsu, J. J. Hansen, K. R. Doty, S. D. Pope, S. T. Smale, T. Hibi, P. B. Rothman, M. Kashiwada, and S. E. Plevy. 2011. NFIL3 is a regulator of IL-12 p40 in macrophages and mucosal immunity. Journal of Immunology 186: 46494655 .

50. Smith, A. M., J. E. Qualls, K. O'Brien, L. Balouzian, P. F. Johnson, S. SchultzCherry, S. T. Smale, and P. J. Murray. 2011. A distal enhancer in Il12b is the target of transcriptional repression by the STAT3 pathway and requires the basic leucine zipper (B-ZIP) protein NFIL3. J Biol Chem 286: 23582-23590.

51. Schaljo, B., F. Kratochvill, N. Gratz, I. Sadzak, I. Sauer, M. Hammer, C. Vogl, B. Strobl, M. Muller, P. J. Blackshear, V. Poli, R. Lang, P. J. Murray, and P. Kovarik. 2009. Tristetraprolin is required for full anti-inflammatory response of murine macrophages to IL-10. Journal of Immunology 183: 1197-1206.

52. Gaba, A., S. I. Grivennikov, M. V. Do, D. J. Stumpo, P. J. Blackshear, and M. Karin. 2012. Cutting edge: IL-10-mediated tristetraprolin induction is part of a 
feedback loop that controls macrophage STAT3 activation and cytokine production. Journal of Immunology 189: 2089-2093.

53. Piessevaux, J., D. Lavens, F. Peelman, and J. Tavernier. 2008. The many faces of the SOCS box. Cytokine Growth Factor Rev 19: 371-381.

54. Berg, D. J., N. Davidson, R. Kuhn, W. Muller, S. Menon, G. Holland, L. Thompson-Snipes, M. W. Leach, and D. Rennick. 1996. Enterocolitis and colon cancer in interleukin-10-deficient mice are associated with aberrant cytokine production and CD4(+) TH1-like responses. The Journal of clinical investigation 98: 1010-1020.

55. Neven, B., E. Mamessier, J. Bruneau, S. Kaltenbach, D. Kotlarz, F. Suarez, J. Masliah-Planchon, K. Billot, D. Canioni, P. Frange, I. Radford-Weiss, V. Asnafi, D. Murugan, C. Bole, P. Nitschke, O. Goulet, J. L. Casanova, S. Blanche, C. Picard, O. Hermine, F. Rieux-Laucat, N. Brousse, F. Davi, V. Baud, C. Klein, B. Nadel, F. Ruemmele, and A. Fischer. 2013. A Mendelian predisposition to B-cell lymphoma caused by IL-10R deficiency. Blood 122: 3713-3722.

56. Dennis, K. L., Y. Wang, N. R. Blatner, S. Wang, A. Saadalla, E. Trudeau, A. Roers, C. T. Weaver, J. J. Lee, J. A. Gilbert, E. B. Chang, and K. Khazaie. 2013. Adenomatous polyps are driven by microbe-instigated focal inflammation and are controlled by IL-10-producing T cells. Cancer Research 73: 5905-5913.

57. Erdman, S. E., J. J. Sohn, V. P. Rao, P. R. Nambiar, Z. Ge, J. G. Fox, and D. B. Schauer. 2005. CD4+CD25+ regulatory lymphocytes induce regression of intestinal tumors in ApcMin/+ mice. Cancer Research 65: 3998-4004. 
58. Dennis, K. L., Y. Wang, N. R. Blatner, S. Wang, A. Saadalla, E. Trudeau, A. Roers, C. T. Weaver, J. J. Lee, J. A. Gilbert, E. B. Chang, and K. Khazaie. 2013. Adenomatous polyps are driven by microbe-instigated focal inflammation and are controlled by IL-10-producing T cells. Cancer Res 73: 5905-5913.

59. Tanikawa, T., C. M. Wilke, I. Kryczek, G. Y. Chen, J. Kao, G. Nunez, and W. Zou. 2012. Interleukin-10 ablation promotes tumor development, growth, and metastasis. Cancer Res 72: 420-429.

60. Yue, F. Y., R. Dummer, R. Geertsen, G. Hofbauer, E. Laine, S. Manolio, and G. Burg. 1997. Interleukin-10 is a growth factor for human melanoma cells and down-regulates HLA class-I, HLA class-II and ICAM-1 molecules. Int J Cancer 71: 630-637.

61. Kurte, M., M. López, A. Aguirre, A. Escobar, J. C. Aguillón, J. Charo, C. G. Larsen, R. Kiessling, and F. Salazar-Onfray. 2004. A synthetic peptide homologous to functional domain of human IL-10 down-regulates expression of MHC class I and Transporter associated with Antigen Processing 1/2 in human melanoma cells. J Immunol 173: 1731-1737.

62. Mumm, J. B., J. Emmerich, X. Zhang, I. Chan, L. Wu, S. Mauze, S. Blaisdell, B. Basham, J. Dai, J. Grein, C. Sheppard, K. Hong, C. Cutler, S. Turner, D. LaFace, M. Kleinschek, M. Judo, G. Ayanoglu, J. Langowski, D. Gu, B. Paporello, E. Murphy, V. Sriram, S. Naravula, B. Desai, S. Medicherla, W. Seghezzi, T. McClanahan, S. Cannon-Carlson, A. M. Beebe, and M. Oft. 2011. IL-10 elicits IFNgamma-dependent tumor immune surveillance. Cancer Cell 20: 781-796. 
63. Emmerich, J., J. B. Mumm, I. H. Chan, D. LaFace, H. Truong, T. McClanahan, D. M. Gorman, and M. Oft. 2012. IL-10 directly activates and expands tumorresident CD8(+) T cells without de novo infiltration from secondary lymphoid organs. Cancer Res 72: 3570-3581.

64. Srivastava, N., R. Sudan, and W. G. Kerr. 2013. Role of inositol polyphosphatases and their targets in T cell biology. Frontiers in immunology 4: 288.

65. Beissert, S., J. Hosoi, S. Grabbe, A. Asahina, and R. D. Granstein. 1995. IL-10 inhibits tumor antigen presentation by epidermal antigen-presenting cells. Journal of Immunology 154: 1280-1286.

66. Asadullah, K., W. Sterry, and H. D. Volk. 2003. Interleukin-10 therapy--review of a new approach. Pharmacol Rev 55: 241-269.

67. Moore, K. W., R. de Waal Malefyt, R. L. Coffman, and A. O'Garra. 2001. Interleukin-10 and the interleukin-10 receptor. Annu Rev Immunol 19: 683-765.

68. Groux, H., M. Bigler, J. E. de Vries, and M. G. Roncarolo. 1996. Interleukin-10 induces a long-term antigen-specific anergic state in human CD4+ T cells. The Journal of experimental medicine 184: 19-29.

69. Steinbrink, K., E. Graulich, S. Kubsch, J. Knop, and A. H. Enk. 2002. CD4(+) and CD8(+) anergic T cells induced by interleukin-10-treated human dendritic cells display antigen-specific suppressor activity. Blood 99: 2468-2476.

70. Zeller, J. C., A. Panoskaltsis-Mortari, W. J. Murphy, F. W. Ruscetti, S. Narula, M. G. Roncarolo, and B. R. Blazar. 1999. Induction of CD4+ T cell alloantigenspecific hyporesponsiveness by IL-10 and TGF-beta. Journal of Immunology 163: 3684-3691. 
71. Steinbrink, K., H. Jonuleit, G. Muller, G. Schuler, J. Knop, and A. H. Enk. 1999. Interleukin-10-treated human dendritic cells induce a melanoma-antigen-specific anergy in CD8(+) T cells resulting in a failure to lyse tumor cells. Blood 93 : $1634-1642$.

72. Yamaguchi, T., and S. Sakaguchi. 2006. Regulatory T cells in immune surveillance and treatment of cancer. Semin Cancer Biol 16: 115-123.

73. Stewart, C. A., H. Metheny, N. Iida, L. Smith, M. Hanson, F. Steinhagen, R. M. Leighty, A. Roers, C. L. Karp, W. Muller, and G. Trinchieri. 2013. Interferondependent IL-10 production by Tregs limits tumor Th17 inflammation. The Journal of clinical investigation 123: 4859-4874.

74. Seo, N., S. Hayakawa, M. Takigawa, and Y. Tokura. 2001. Interleukin-10 expressed at early tumour sites induces subsequent generation of CD4(+) Tregulatory cells and systemic collapse of antitumour immunity. Immunology 103: $449-457$.

75. Cottrez, F., and H. Groux. 2001. Regulation of TGF-beta response during T cell activation is modulated by IL-10. Journal of Immunology 167: 773-778.

76. Beury, D. W., K. H. Parker, M. Nyandjo, P. Sinha, K. A. Carter, and S. OstrandRosenberg. 2014. Cross-talk among myeloid-derived suppressor cells, macrophages, and tumor cells impacts the inflammatory milieu of solid tumors. $J$ Leukoc Biol 96: 1109-1118.

77. Sredni, B., M. Weil, G. Khomenok, I. Lebenthal, S. Teitz, Y. Mardor, Z. Ram, A. Orenstein, A. Kershenovich, S. Michowiz, Y. I. Cohen, Z. H. Rappaport, I. Freidkin, M. Albeck, D. L. Longo, and Y. Kalechman. 2004. Ammonium 
trichloro(dioxoethylene-o,o')tellurate (AS101) sensitizes tumors to chemotherapy by inhibiting the tumor interleukin 10 autocrine loop. Cancer Research 64: 18431852.

78. Alas, S., C. Emmanouilides, and B. Bonavida. 2001. Inhibition of interleukin 10 by rituximab results in down-regulation of bcl-2 and sensitization of B-cell nonHodgkin's lymphoma to apoptosis. Clin Cancer Res 7: 709-723.

79. Kim, J., R. L. Modlin, R. L. Moy, S. M. Dubinett, T. McHugh, B. J. Nickoloff, and K. Uyemura. 1995. IL-10 production in cutaneous basal and squamous cell carcinomas. A mechanism for evading the local $\mathrm{T}$ cell immune response. Journal of Immunology 155: 2240-2247.

80. Itakura, E., R. R. Huang, D. R. Wen, E. Paul, P. H. Wunsch, and A. J. Cochran. 2011. IL-10 expression by primary tumor cells correlates with melanoma progression from radial to vertical growth phase and development of metastatic competence. Mod Pathol 24: 801-809.

81. Al-Huseini, L. M., H. X. Aw Yeang, S. Sethu, N. Alhumeed, J. M. Hamdam, Y. Tingle, L. Djouhri, N. Kitteringham, B. K. Park, C. E. Goldring, and J. G. Sathish. 2013. Nuclear factor-erythroid 2 (NF-E2) p45-related factor-2 (Nrf2) modulates dendritic cell immune function through regulation of p38 MAPK-cAMPresponsive element binding protein/activating transcription factor 1 signaling. $J$ Biol Chem 288: 22281-22288.

82. Saraiva, M., and A. O'Garra. 2010. The regulation of IL-10 production by immune cells. Nat Rev Immunol 10: 170-181. 
83. Huang, G., L. Z. Shi, and H. Chi. 2009. Regulation of JNK and p38 MAPK in the immune system: signal integration, propagation and termination. Cytokine 48: 161-169.

84. Shiao, S. L., A. P. Ganesan, H. S. Rugo, and L. M. Coussens. 2011. Immune microenvironments in solid tumors: new targets for therapy. Genes Dev 25: 25592572.

85. Cao, C., Y. Gu, C. Zhu, T. Palmai-Pallag, F. Lan, Z. Chen, W. Li, H. Shen, and S. Ying. 2014. Potential roles of eosinophils in cancer therapy: epidemiological studies, experimental models, and clinical pathology. Recent Pat Anticancer Drug Discov 9: 241-248.

86. Khazaie, K., N. R. Blatner, M. W. Khan, F. Gounari, E. Gounaris, K. Dennis, A. Bonertz, F. N. Tsai, M. J. Strouch, E. Cheon, J. D. Phillips, P. Beckhove, and D. J. Bentrem. 2011. The significant role of mast cells in cancer. Cancer Metastasis $\operatorname{Rev} 30: 45-60$.

87. Saleem, S. J., R. K. Martin, J. K. Morales, J. L. Sturgill, D. R. Gibb, L. Graham, H. D. Bear, M. H. Manjili, J. J. Ryan, and D. H. Conrad. 2012. Cutting edge: mast cells critically augment myeloid-derived suppressor cell activity. J Immunol 189: 511-515.

88. Danelli, L., B. Frossi, G. Gri, F. Mion, C. Guarnotta, L. Bongiovanni, C. Tripodo, L. Mariuzzi, S. Marzinotto, A. Rigoni, U. Blank, M. P. Colombo, and C. E. Pucillo. 2015. Mast cells boost myeloid-derived suppressor cell activity and contribute to the development of tumor-favoring microenvironment. Cancer Immunol Res 3: 85-95. 
89. Martin, R. K., S. J. Saleem, L. Folgosa, H. B. Zellner, S. R. Damle, G. K. Nguyen, J. J. Ryan, H. D. Bear, A. M. Irani, and D. H. Conrad. 2014. Mast cell histamine promotes the immunoregulatory activity of myeloid-derived suppressor cells. J Leukoc Biol 96: 151-159.

90. Conti, P., D. Kempuraj, M. Di Gioacchino, W. Boucher, R. Letourneau, K. Kandere, R. C. Barbacane, M. Reale, M. Felaco, S. Frydas, and T. C. Theoharides. 2002. Interleukin-6 and mast cells. Allergy Asthma Proc 23: 331335.

91. Lippitz, B. E. 2013. Cytokine patterns in patients with cancer: a systematic review. Lancet Oncol 14: e218-228.

92. Bunt, S. K., L. Yang, P. Sinha, V. K. Clements, J. Leips, and S. OstrandRosenberg. 2007. Reduced inflammation in the tumor microenvironment delays the accumulation of myeloid-derived suppressor cells and limits tumor progression. Cancer Res 67: 10019-10026.

93. Smith, C., M. Y. Chang, K. H. Parker, D. W. Beury, J. B. DuHadaway, H. E. Flick, J. Boulden, E. Sutanto-Ward, A. P. Soler, L. D. Laury-Kleintop, L. Mandik-Nayak, R. Metz, S. Ostrand-Rosenberg, G. C. Prendergast, and A. J. Muller. 2012. IDO is a nodal pathogenic driver of lung cancer and metastasis development. Cancer Discov 2: 722-735.

94. Marigo, I., E. Bosio, S. Solito, C. Mesa, A. Fernandez, L. Dolcetti, S. Ugel, N. Sonda, S. Bicciato, E. Falisi, F. Calabrese, G. Basso, P. Zanovello, E. Cozzi, S. Mandruzzato, and V. Bronte. 2010. Tumor-induced tolerance and immune suppression depend on the C/EBPbeta transcription factor. Immunity 32: 790-802. 
95. Wruck, C. J., K. Streetz, G. Pavic, M. E. Gotz, M. Tohidnezhad, L. O. Brandenburg, D. Varoga, O. Eickelberg, T. Herdegen, C. Trautwein, K. Cha, Y. W. Kan, and T. Pufe. 2011. Nrf2 induces interleukin-6 (IL-6) expression via an antioxidant response element within the IL-6 promoter. J Biol Chem 286: 44934499.

96. Frossi, B., M. De Carli, K. C. Daniel, J. Rivera, and C. Pucillo. 2003. Oxidative stress stimulates IL-4 and IL-6 production in mast cells by an APE/Ref-1dependent pathway. European journal of immunology 33: 2168-2177. 
Appendix 1: Cross-talk among myeloid-derived suppressor cells, macrophages, and tumor cells impacts the inflammatory milieu of solid tumor 
Epub ahead of print August 28, 2014 - doi:10.1189/jlb.3A0414-210R

JLB

Article

\title{
Cross-talk among myeloid-derived suppressor cells, macrophages, and tumor cells impacts the inflammatory milieu of solid tumors
}

\author{
Daniel W. Beury, Katherine H. Parker, Maeva Nyandjo, Pratima Sinha, Kayla A. Carter,
} and Suzanne Ostrand-Rosenberg ${ }^{\prime}$

Department of Biological Sciences, University of Maryland, Baltimore County, Baltimore, Maryland, USA RECFIVED APRIL. 17, 2014; REVISED AUCUST 4, 2014; ACCYPIED AUCUST 11, 2014. DOL: 10.1189/jb.3M0414210R

\begin{abstract}
MDSC and macrophages are present in most solld tumors and are important drivers of immune suppression and Inflammation. It is establlshed that cross-talk between MDSC and macrophages impacts antl-tumor Immunity; however, Interactions between tumor cells and MDSC or macrophages are less well studled. To examine potentlal Interactions between these cells, we studled the Impact of MDSC, macrophages, and four murine tumor cell IInes on each other, both in vitro and in vivo. We tocused on IL-6, IL-10, IL-12, TNF- $\alpha$, and NO, as these molecules are produced by macrophages, MDSC, and many tumor cells; are present in most solld tumors; and regulate Inflammation. In vitro studles demonstrated that MDSC-produced IL-10 decreased macrophage IL-6 and TNF- $\alpha$ and Increased NO. IL-6 Indirectly regulated MDSC IL-10. Tumor cells Increased MDSC IL-6 and vice versa. Tumor cells also Increased macrophage IL-6 and NO and decreased macrophage TNF- $\alpha$. Tumor cell-driven macrophage IL-6 was reduced by MDSC, and tumor cells and MDSC enhanced macrophage NO. In vivo analysis of solld tumors Identifled IL- 6 and IL-10 as the dominant cytoklnes and demonstrated that these molecules were produced predominantly by stromal cells. These results suggest that Inflammation within solld tumors is regulated by the ratio of tumor cells to MDSC and macrophages and that Interactions of these cells have the potentlal to alter significantly the inflammatory milleu within the tumor microenvironment. J. LeukOC. Blol. 96: 000-000; 2014.
\end{abstract}

\section{Introduction}

Solid tumors are a complex and frequently inflamed environment. The inflammation is driven by proinflammatory media-

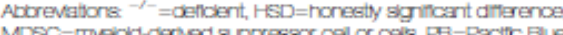

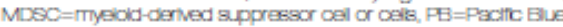
UMB=Universty of Maryand Eatimore, UMBC=Universty of Maryland Batmore County. VEGF=vascular endothelal gowth factor, WT=wid-type

The orite version of this paper, tound at wwwieukbougg indudes suppiernentaintornation. tors that are secreted by tumor cells, various tumor-infiltrating lymphocytes, tumor-associated fibroblasts, and myeloid cells, such as macrophages, dendritic cells, and MDSC [1]. Some of these cells engage in cross-talk with each other, resulting in the release of proinflammatory cytokines (e.g., IL-1, IL-6, IL17, TNF- $\alpha$ ), chemokines (e.g., CCL.2, CXCL.5, CXCL12), growth factors (e.g., TGF- $\beta$, GM-CSF, VEGF), and other effector molecules (c.g., S100A8/A9, high-mobility group box 1) [2-4]. These factors, in turn, induce the accumulation and enhance the function of immune-suppressive cells, such as regulatory T cells, plasmacytoid dendritic cells, tumor-associated macrophages, and MDSC [3, 5, 6]. Although the cellular interactions contributing to some of the protumor factors present in the tumor microenvironment have been identified, the etiology of others remains unknown.

Macrophages and MDSC are present within most solid tumors, where they are major drivers of immune suppression and inflammation [3]. We have reported previously that these cells participate in cross-talk with each other that results in increased MDSC production of IL-10 and decreased macrophage production of II-12, thereby polarizing the immune system toward a protumor type 2 environment $[7,8]$. Additional factors are also likely to be impacted by cross-talk between MDSC and macrophages, as well as by interactions with tumor cells. Therefore, we have investigated how tumor cells, macrophages, and MDSC interact with respect to II-6, TNF- $\alpha$, II-10, and NO. We have focused on these four molecules, as they are chronically present in many solid tumors and play important roles in tumor progression. IL-6 promotes tumor progression by enhancing tumor cell development, growth, and metastasis and by inhibiting apoptosis and enhancing tumor vascularization [9-11]. TNF- $\alpha$ causes DNA damage, inhibits apoptosis, and induces the production of matrix metalloproteases, cytokines, and chemokines that facilitate tumor cell invasion and metastasis [12]. In contrast to IL-6 and TNF- $\alpha$, which when chronically present, are exclusively protumor, NO can have pro- and anti-tumor activity. When produced by M1-like macrophages, NO induces tumor cell apoptosis [13]. However,

\footnotetext{
1. Correspondence: Depe of Biological Sciences, UMBC, 1000 Hilltop Circle, Baltimore, MD 21250, USA. E-mail: srosenbedumbc.edu
}

Volume 96, December 2014 Jaumal or Leukocyte Blology 1 
when produced by MDSC, NO drives immune suppression [14]. II-10 has also been associated with pro- and anti-tumor activity [15]. Here, we report that macrophages, MDSC, and tumor cells participate in a network of cross-talk, resulting in differential production of IL-6, IL-10, TNF- $\alpha$, and NO, suggesting that the interaction of these cells has the potential to alter significantly the inflammatory milieu within the tumor microenvironment.

\section{MATERIALS AND METHODS}

\section{Mice, tumor cells, tumor growth}

$\mathrm{BAL} / \mathrm{C}, \mathrm{CS7BL} / 6$, BALB/C IL-6- ${ }^{-1-}$, and BAL.B/c II- $10^{-/-}$mice were hred in the UMBC animal farility from stock obtained from The Jackson Lahoratory (Bar Harbor, ME, USA; C57BL_/6 and BAL.B/c) or provided by Dr. Manfred

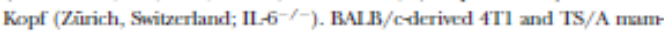
mary carcinoenas, CT26 colon carcinoma, and C57BL./6-derived MC38 colon carcinoma were maintained as described [16]. Mice were inoculated in the abdominal mammary gland with $100 \mu \mathrm{l}$ DMFM containing $7 \times 10^{9}$ (WT and II- $10^{-/}$- mice) or $10^{5}$ (WT and II- $-6^{-1-}$ mice) $4 \mathrm{Tl}$ cells or $10^{p} \mathrm{TS} / \mathrm{A}$ cells or s.c. in the flank with $5 \times 10^{5}, 1 \times 10^{4}$, or $1 \times 10^{4}$ Cr26 cells. Primary tumors were measured as described [17]. Survival time was recorded when mice be came moribund and were euthanized. All animal procedures were approved by the UMBC Institutional Animal Care and Use Committec.

\section{Flow cytometry and antibodies}

Gr1-FTTC, Gr1-allophycocyanin, Ly6C-FTTC, Ly6G-PB, CD11b-PE, CD11bPB, F4/80-allophycocyanin, F4/80-PB, pSTAT3-PB, II-6R-PE, and II-10R-PE $\mathrm{mAb}$ and rat IgG1-PE and IgG2b-PE isotypes were from BD PharMingen (San Diego, CA, USA) or Biolegend (San Dicgo, CA, USA). Cells were stained for surface markers as described [18]. For phosphoflow experiments, cells were stimulated with $50 \mathrm{ng} / \mathrm{mL}$. rll-10 (Biol.egend) or supernatants from MDSC and macrophage cocultures, fixed with Lyse/Fix Buffer (BD Biosciences, San Jose, CA, USA), permeabilized with Perm Buffer III (BD Bicsciences), and stained with antibodies diluted in Stain Buffer (BD) Biosciences). Samples were analyzed on a Beckman/Coulter CyAn ADP flow cytometer using Summit software.

\section{T Cell proliferation assays}

$\mathrm{CD}^{+}$and $\mathrm{CDS}^{+} \mathrm{T}$ cell proliferation assays were performed as described [18]. Briefly, DO11.10 (ovalbumin peptide $\varepsilon_{23--a 0}$-specific, $1-A^{\mathrm{d}}-$-restricted) or Clone 4 (hemagglutinin peptide ${ }_{515-5}$-specific, H-2K ${ }^{d}$-restricted) splenocytes were cultured with their respective cognate peptides and irradiated blood MDSC from 4T1-bearing WT, $11--^{-1-}$, or II- $10^{-1-}$ mice. Cultures were pulsed with "H-thymidine on Day 4 and harvested on Day 5. Peptides were synthesized at the UMB Biopolymer Core Facility.

\section{MIDSC, macrophage, MIDS-macrophage-tumor cell crosstallk}

MDSC were isolated from the peripheral blood of $4 \mathrm{~T} 1$ tumorbearing mice [16]. Peritoneal macrophages were prepared froen tumor-free mice [8]. MDSC and marrophages in all experiments were $>90 \% \mathrm{Grl}^{+} \mathrm{CD}_{11 \mathrm{~b}^{+}}$cells and $>95 \% \mathrm{CD}^{2} \mathrm{bb}^{+} \mathrm{F} 4 / 80^{+}$cells, respectively, as assessed by flow cytometry. MDSC and macrophage cross-talk experiments were performed as described [7] with the following modificativen: 4T1, MC58, TS/A, or CT26 tumor cells ( $1 \times 10^{5}$ cells) were cultured with or without $7.5 \times 10^{5} \mathrm{MDSC}$ and/or macrophages in $500 \mu \mathrm{l}$ mactophage media (5\% FCS in DMEM, $1 \%$ penicillinstrep tomycin, $1 \%$ glutamax, $0.1 \%$ gentamycin) for $16 \mathrm{~h}$ at $37 \mathrm{C}$ with $100 \mathrm{ng} / \mathrm{mL}$. LSS (Difco Lahoratories, Franklin Lakes, NJ, USA) and $20 \mathrm{U} / \mathrm{mL}$. FN- $\gamma$ (R\&D Systems, Minneapolis, MN, USA). In some experiments, macrophages and/or MDSC were cultured with L.PS, IFN-y, rll-6 and rIl-10 (boch from Biolesg end) and II-10 that was denatured by boiling at $95^{\circ} \mathrm{C}$ for $15 \mathrm{~min}$ or in the presence of neutralizing antihodies to II-10 (1 $\mu \mathrm{g} / \mathrm{ml}$; Clone JFS5-2A5; eBio- science, San Diego, CA, USA). Cells were harvested by scraping and analyzed by flow cytometry. Supernatants were analyzed for II-10, II-6, and TNF a wing EIISA kits (R\&D Systems and eBioscience), per the manufacturers' protecol, or by multiplex analysis in the UMB Cytokine Core Farility. NO producticen was quantified by Griess assay [18]. Values were normalized between experiments using the following formulas

- production of IL-6 by macrophages or MDSC in response to tumor cells - (II-6 from WT MDSC or macrophages with tumor cells) (II-6 from II.-6 - $^{-1}$ macrophage or MDSC with tumor celk)

- percent increase in II-6 or NO by MDSC or macrophages in response to tumor cells - I[(II- -6 or NO from macrophages and MDSC \pm tumor cells) $/$ (II-6 or NO from macrophages or MDSC) ] $\times 100 \%\}-100 \%$

- percent decrease in IL-6 or TNF- $\alpha$ by macrophages in response to thmor cells and/or MDSC $-1-[$ (II-6 or TNF- $\alpha$ from macrophages \pm tumor cells) $/$ (IL-6 or TNF- $\alpha$ from WT macrophages \pm tumor cells \pm MDSC) $] \times 100 \%$

- percent increase in II-10 by MDSC in response to mactophages - II(II-10 from macrophaggr +MDSC)/(II-10 from MDSC) ] $\times 100 \%$ ) $-100 \%$ If II-6 was not detected, then the lowest value detectable on the standard curve was used for the calculations.

Macrophages and MDSC were stained with $5 \mu \mathrm{M}$ CellTrace Violet (Life Technologies, Carlsbad, CA, USA) and $4 \mathrm{~T} 1$ tumor cells with $1 \mu \mathrm{M}$ CFSE (Life Technologies). MDSC or macrophages were cultured for $16 \mathrm{~h}$ in macrophage media with $100 \mathrm{ng} / \mathrm{mL}$. L.PS and $20 \mathrm{U} / \mathrm{mL}$. IFN- $\gamma$ in a six-well dish at $3 \times 10^{5}$ cells $/$ well $/ 2 \mathrm{~mL}$, with or without $4 \times 10^{5} 4 \mathrm{Tl}$ cells. Cells were then harvested using Detachin (Genlantis, San Diego, CA, USA) and scraping, washed, and stained for Gr1, CD11b, and with 7-amincractinomycin D; and analyzed by flow cytometry.

\section{Ex vivo tumor cultures}

$4 T 1, C T 26$, and TS/A tumoes $>8 \mathrm{~mm}$ in diameter were surgically resected froen euthanized mice and placed on sterile 450 Whatman filter paper to remove excess liquid. The tumors were then transferred to $6 \mathrm{~cm}$ culture dishes and fincly minced using a sterile scalpel, and the resulting pieces wcighed. $4 \mathrm{Tl}$ and TS/A pieces were resuspended in $5 \mathrm{~mL}$. prewarmed $4 \mathrm{Tl}$ media (10\% Fetal Cone I in IMDM, $1 \%$ penicillin-streptoenycin, $1 \%$ glutarmax, $0.1 \%$ gentamycin) containing $100 \mathrm{ng} / \mathrm{mL}$. L.PS and $20 \mathrm{U} / \mathrm{mL}$. IFN-y for II-10 studies or without LXS and IFN-y for IL-6 studies. Resuspended tumor pieces were incubated for $16 \mathrm{~h}$ at $37 \mathrm{C}^{\circ}, 5 \% \mathrm{CO}_{2}$, and supernatants were analyzed for cotokine production by E.ISA. Cytokine levels were normalized to one gram of turnor tisue/ml. media using the following formulk ogtokine prochurtion (normalised) - cytokine $(\mathrm{pg} / \mathrm{mL}$.) $\times[$ (tumor weight/ $1 \mathrm{~g}) \times 5 \mathrm{~mL}$.$] .$

\section{Statistical analyses}

Student's ftest and Tukey's HSD test were performed using Microsoft Excel 2013. Values denoted with different letters (e.g., a, b, c, etc.) are significantly different from each other, values with the same letter are not significantly different. Tumor growth and exogenous IL-10 data were analyzed using the Mann-Whitncy test on the VissarStats wehsite (www.VassarStats.net). Survival data were analyzed using the log-rank test from the Walter and Eliza Hall Institute of Medical Rescarch Bioinformatics webpage (http:// bioinf.wchiedu.au/software/russell//logrank/). Values of $P<0.05$ were considered statistically significant.

\section{RESULTS}

\section{IL-6 and IL-10 promote tumor progression}

Increased levels of serum IL-6 are correlated with chronic inflammation, increased tumor burden, and poor prognosis in some human and mouse systems [19]. IL-6 also promotes MDSC-mediated inhibition of Th1 responses in mice [20]. In contrast, IL-10 correlates with tumor progression in some systems but with tumor regression in other systems [15, 21-25]. 
To determine if IL-6 and/or IL-10 contribute to progression of the 4T1 mammary carcinoma or CT26 colon carcinoma, we inoculated syngeneic WT, IL- $6^{-/-}$, and IL-10 $10^{-/}$mice with 4T1 (Fig. 1A) or CT26 (Fig. 1B) tumor cells and followed the mice for tumor onset, growth, and engrafument. In the absence of host-produced IL-6, 4T1 tumor progression was delayed, and survival time was increased. IL- $10^{-/-}$mice showed a similar, although less dramatic, delay in tumor progression and extension of survival time. 4T1 tumor engrafument in WT $\mathrm{BAL} B / \mathrm{c}$ and $\mathrm{IL}-10^{-/-}$mice was $90-100 \%$, whereas only $40 \%$ of IL- $-6^{-/-}$mice developed tumor. Tumor progression was also delayed, and survival time increased in $\mathrm{IL}-6^{-/-}$mice with CT26 tumors. In contrast, IL-10 $0^{-/-}$mice inoculated with $5 \times$ $10^{5} \mathrm{CT} 26$ tumor cells had similar tumor progression, survival time, and percent engraftment as WT mice. Tumor progression, survival time, and engraftment were also similar in WT and IL-10 $0^{-/-}$BALB/c mice inoculated with $1 \times 10^{5}$ or $1 \times$ $10^{4}$ cells (Supplemental Fig. 1A). These results demonstrate that stromal cell-derived IL-6 and IL-10 facilitate progression of 4T1 and CT26 tumors in their syngeneic hosts.

\section{MDSC production of IL-10 decreases macrophage IL-6 and TNF- $\alpha$ and increases NO; IL-6 indirectly regulates MDSC production of $\mathrm{IL}-10$}

We have shown previously that MDSC production of IL-10 is enhanced by cross-talk with macrophages and polarizes macrophages toward a tumor-promoting phenotype by inhibiting macrophage production of IL-12 [7, 8]. To determine if IL-10 produced by MDSC impacts the production of additional proinflammatory mediators, we cocultured $\mathrm{CD} 11 \mathrm{~b}^{+} \mathrm{F} 4 / 80^{+}$peritoneal macrophages and $4 \mathrm{~T} 1$-induced $\mathrm{Gr}^{+}{ }^{+} \mathrm{CD} 11 \mathrm{~b}^{+}$immunesuppressive MDSC (Fig. 2A) and assayed the supernatants for IL-10 and the proinflammatory cytokine IL-6 (Fig. 2B). Consistent with our previous reports, production of IL-10 was increased significantly in the presence of macrophages (average increase in IL-10 of $116 \pm 19.4 \%$ for 30 experiments). IL-10 was produced exclusively by MDSC, as macrophage cultures containing IL-10 $10^{-/-}$MDSC produced no IL-10. In the same cocultures, macrophages were the sole producers of IL -6 , and MDSC decreased macrophage IL-6 (average decrease in IL-6 of $24 \pm 3.8 \%$ for 30 experiments).

To determine if IL-6 regulates MDSC production of IL-10, we cocultured WT or IL- $6^{-/-}$macrophages with WT or IL$6^{-/-}$MDSC (Fig. 2C). IL-6 ${ }^{-/-}$MDSC produced significantly more IL-10 than WT MDSC. Macrophage cocultures with IL$6^{-/-}$MDSC had significantly more IL-10 than cocultures with WT MDSC. Macrophage IL-6 had no effect on MDSC IL-10, as WT MDSC cocultured with WT or IL- $6^{-/-}$macrophages produced similar amounts of IL-10. The lack of a direct effect by IL-6 on MDSC IL-10 was confirmed by incubation of MDSC with exogenous IL-6 (Supplemental Fig. 1B). These results indicate that MDSC do not produce IL-6 in the coculture setting; however, their development in vivo in the presence of IL-6 down-regulates their production of IL-10.

To determine if IL-10 produced by MDSC decreased macrophage IL-6 or regulated other molecules characteristic of tumorrejecting M1 macrophages, WT or II-10 $0^{-/-}$MDSC were cocultured with WT macrophages (Fig. 2D). There was no decrease in
A
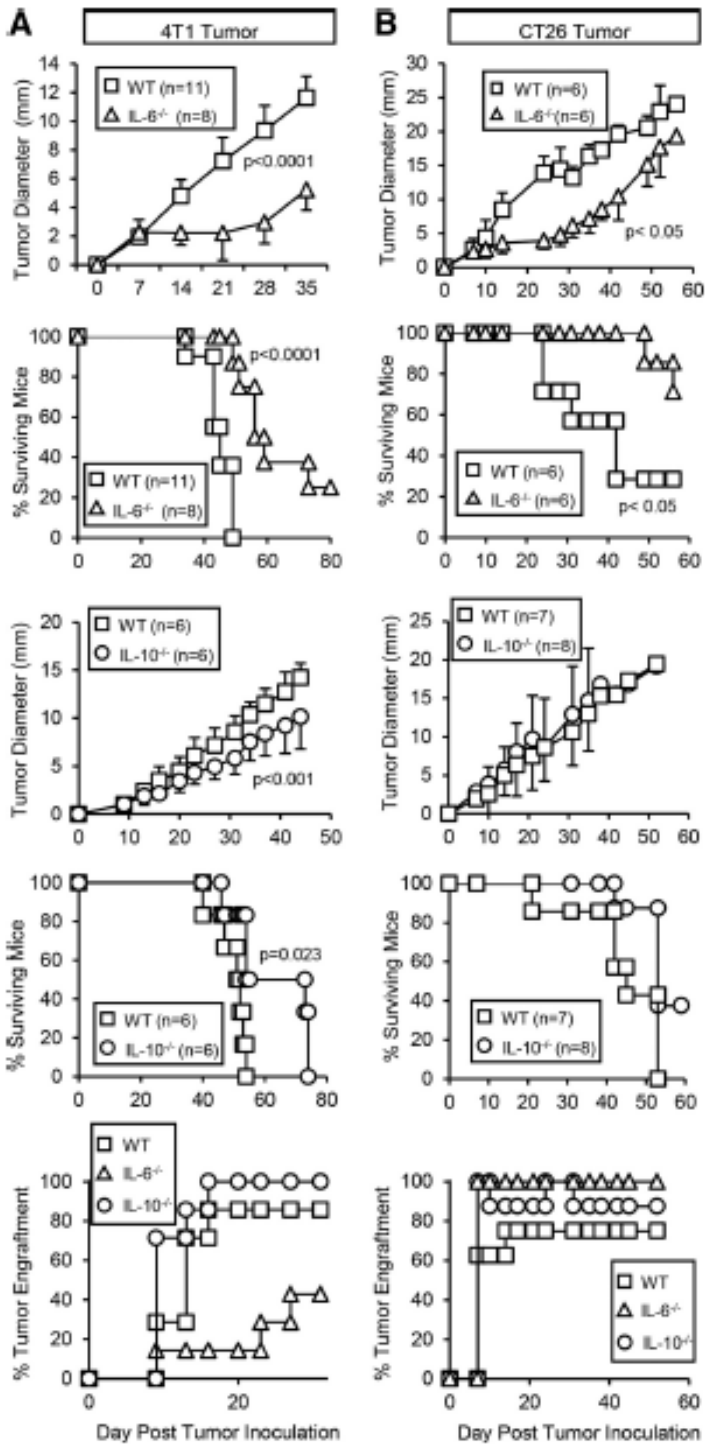

Figure 1. II-6 and IL-10 produced by host cells enhance primary tumor growth and decrease survival time. WT, IL- $6^{-/-}$, and IL $-10^{-1-}$ $\mathrm{BAL} . \mathrm{B} / \mathrm{c}$ mice were inoculated with (A) $4 \mathrm{~T} 1$ or (B) CT26 turnor cells and monitored for tumor diameter, survival, and tumor engraftment. Mice in the WT versus IL- $6^{-/-}$graphs and WT versus IL- $10^{-/-}$graphs (tumor diameter and percent survival) were inoculated with $1 \times 10^{5}$ and $70004 \mathrm{~T} 1$ cells, respectively. Mice in the engraftment graph were inoculated with $1 \times 10^{5} 4 \mathrm{~T} 1$ cells. All CT26 inoculations were $5 \times 10^{5}$ cells. For tumor engraftment, $\mathrm{n}=7$ for each $4 \mathrm{~T} 1$ group; $\mathrm{n}=6$ for each CT26 group. Statistical significance was tested by Mann-Whitney (tumor growh) or log-rank test (survival). Data are pooled from three independent experiments. 

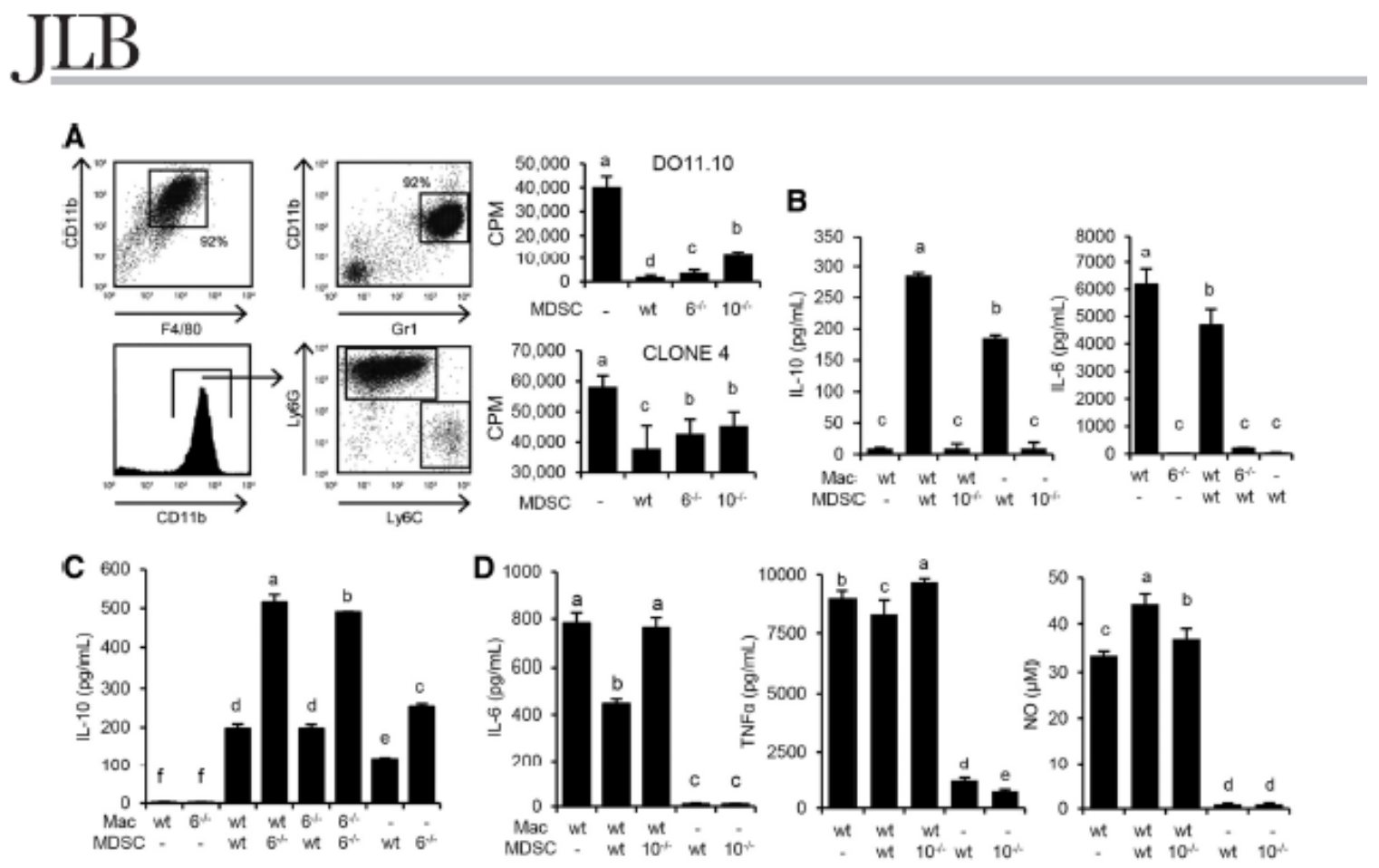

E
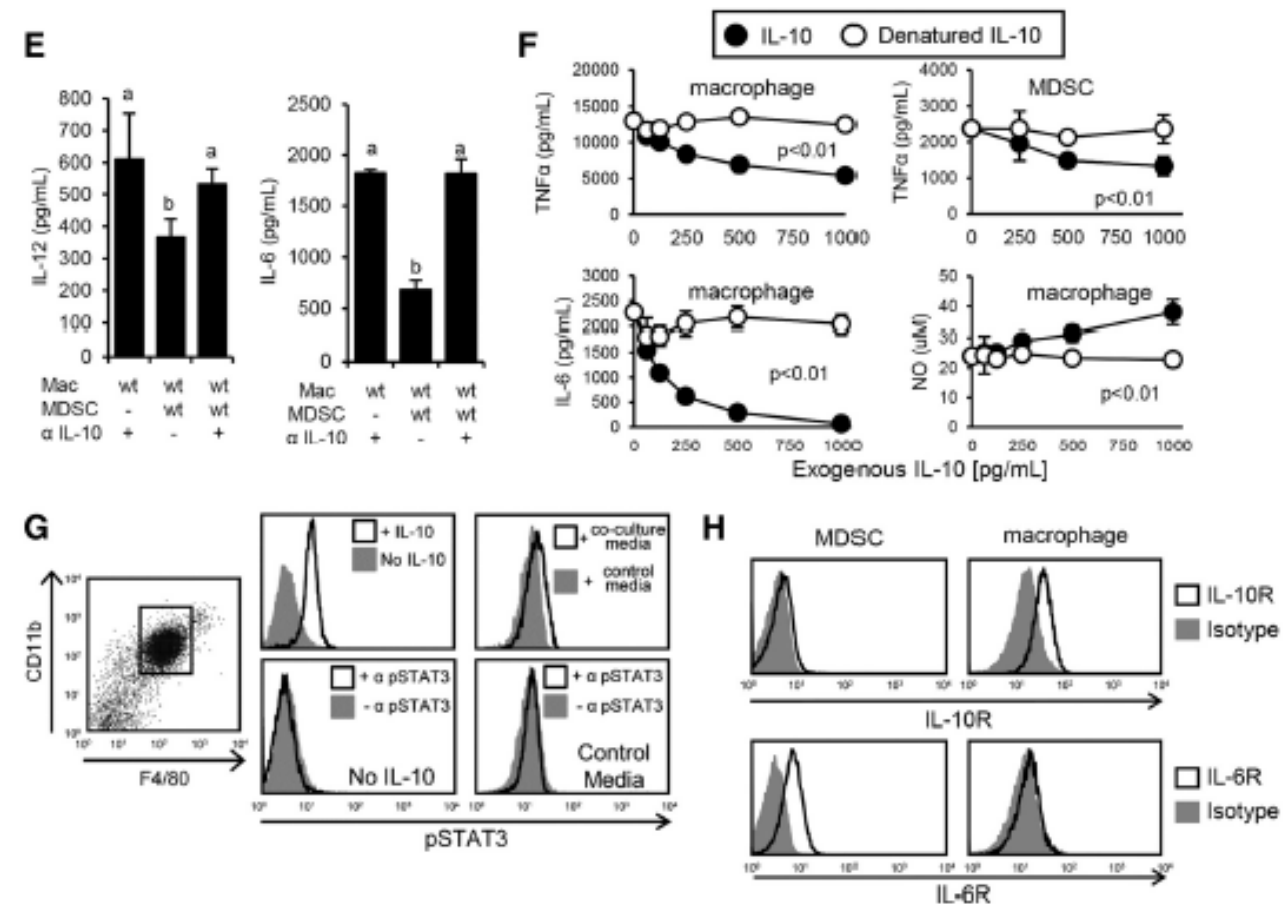

Figure 2. Cross-talk between MDSC and macrophages regulates production of IL-10, IL-6, and NO. (A) Peritoneal macrophages from healthy mice and MDSC from tumor-bearing mice were stained with mAb to CD11b, F4/80, Gr1, Ly6C, and/or Ly6G and analyzed by flow cytometry. 
IIL-6 in the presence of IL-10 $10^{-/-}$MDSC, suggesing that IL- 10 from WT MDSC reduced macrophage IL- 6 . To confirm the role of IL-10, neutralizing antibodies to IL-10 were added to MDSCmacrophage cocultures. As previous studies demonstrated that MDSC IL-10 also decreases macrophage IL-12 [8], IL-12 levels served as a positive control (Fig. 2E). IL-10 neutralizing antibodies reduced the MDSC-mediated decrease of IL-6 and IL-12. Thus, a feedback loop exists between macrophages and MDSC, in which macrophages increase MDSC production of IL-10, and MDSC IL-10 regulates macrophage synthesis of IL-6.

We also assessed the role of MDSC IL-10 on macrophage NO and TNF- $\alpha$ production (Fig. 2D). MDSC IL-10 decreased TNF- $\alpha$ in the cocultures; however, this decrease was minimal. In contrast, macrophage production of NO was increased by coculture with MDSC. The increase was predominantly a result of MDSC IL-10, as only a minimal increase in NO was observed in the presence of $\mathrm{IL}-10^{-/-}$MDSC.

To confirm further that IL-10 regulated macrophage production of IL-6 and NO, and macrophage and MDSC production of TNF- $\alpha$, macrophages or MDSC were cultured in the presence of exogenous IL-10, and culture supernatants were assessed for TNF- $\alpha$, IL-6, and NO (Fig. 2F). Exogenous IL-10 reduced MDSC and macrophage TNF- $\alpha$ and macrophage IL -6 but increased macrophage NO. As STAT3 is activated by signaling through II-10R, macrophages were cultured with exogenous IL-10 or with supernatants from MDSC-macrophage cocultures and subsequently stained for phosphorylated STAT3 (Fig. 2G). STAT3 was phosphorylated under both conditions, further confirming the regulatory role of IL-10 produced by MDSC.

MDSC and macrophages express IL-6R and IL-10R, respectively (Fig. 2H), so these cells have the potential to respond directly to these cytokines. The results of Fig. $2 \mathrm{~F}$ suggest that IL-10 directly impacts macrophages. However, IL-10-deficiency and IL-6-deficiency could also cause other changes in MDSCs and/or macrophages so that the effects are only mediated indirectly by IL-10 or II-6. To distinguish these possibilities, we compared cytokine/ chemokine production by WT, IL-10 $10^{-/}$, and IL- $6^{-/-}$MDSC to determine if gene deficiency impacts MDSC phenotype (Supplemental Table 1). TGF- $\beta 3$, GM-CSF, IL-4, IL-13, and IL-23 were not detectable in WT MDSC. TGF $\beta 2$, IL-1- $\beta$, OCL 2 , and VEGF production was similar for WT, IL-10 $10^{-1-}$, and IL- $6^{-/-}$MDSC. TGF- $\beta 1$ trended higher in IL- $10^{-/-}$and IL- $6^{-/-}$MDSC, and MIP-1 $\alpha$ trended lower in IL-10 $10^{-/-}$and IL $-6^{-/-}$MDSC compared with WT MDSC. These results suggest that II-10-deficiency and IL-6-deficiency may alter the phenotype of MDSC.

These results, together with our earlier studies on IL-12 [7, 8], demonstrate that MDSC production of IL-10 increases some M2-like characteristics of macrophages (i.e., IL-12 $2^{\mathrm{lom}} \mathrm{IL}$ $6^{\text {low }}$ ) but also increases some M1-like properties $\left(\mathrm{NO}^{\text {hight }}\right)$.

\section{Other cytokines are also impacted by interactions between MDSC and macrophages}

In addition to IL-10, TNF- $\alpha$, IL-12, NO, and IL -6 , other immune-regulatory molecules are present in solid tumors. Of particular note are cytokines that drive effector and regulatory T cells (e.g., IL-23, IL-27, IL-4, and IL-13), growth factors that regulate neovascularization (e.g., VEGF) and myeloid cell differentiation (e.g., GM-CSF), proinflammatory mediators (e.g., IL-1 $\beta$ ), and immune-suppressive molecules (e.g., TGF- $\beta$ ). To determine if any of these molecules are affected by cross-talk between MDSC and macrophages, supernatants from cocultures of 4T1-induced WT MDSC and WT BALB/c macrophages were assayed by multiplex analysis (Supplemental Table 1). Neither MDSC nor macrophages produced TGF- $\beta 3$, GM-CSF, IL-4, IL-13, or IL-23, whereas both cell types produced TGF- $\beta 1$, TGF- $\beta 2$, IL- $1 \beta$, CCL 2 , MIP- $1 \alpha$, and VEGF. Cocultures using WT MDSC reduced the production of TGF- $\beta 1$, TGF- $\beta 2$, and MIP-1 $\alpha$ and modestly increased the production of VEGF. Cocultures of WT macrophages with IL-10 $0^{-/-}$or IL$6^{-/-}$MDSC displayed similar trends, except for CCL2, where we observed a decrease in CCL.2 production.

\section{Tumor cells increase MDSC production of IL-6 and vice versa}

Tumor cells produce proinflammatory mediators and therefore, may contribute to the polarization of myeloid cells in the tumor microenvironment. To assess if there is cross-talk between MDSC and tumor cells, 4T1, CT26, TS/A, or MC38 murine tumor cells were cultured by themselves or cocultured with MDSC (Fig. 3). When cultured alone, 4T1 and CT26 cells produced IL-6, and TS/A, MC38, and MDSC produced no detectable IL-6. Cultures containing WT MDSC plus 4T1, CT26, TS/A, or MCS8 tumor cells contained more IL-6 than cultures of tumor cells alone, whereas cultures of $4 \mathrm{~T} 1$, CT26, and TS/A tumor cells plus IL-6 $6^{-/-}$MDSC produced intermediate

MDSC from WT, IL-10 $0^{-/-}$, and IL-6 ${ }^{-/-}$BAL.B/c mice with 4T1 tumors were assayed for their ability to suppress the antigen-driven activation of peptide-specific, MHC-restricted, transgenic $\mathrm{CD}^{+}$(DO11.10) and $\mathrm{CD}^{+}$(Clone 4) $\mathrm{T}$ cells. (B-D) 4T1-induced MDSC and peritoneal macrophages (Mac) from WT, IL-10-1- $\left(10^{-/-}\right)$, or IL- $6^{-/-}\left(6^{-/-}\right)$BALB/c mice were cocultured, and supernatants were assayed for IL-10, IL-6, and NO. (B) Macrophages enhance MDSC IL-10, and MDSC decrease macrophage IL-6. (C) IL-6 decreases MDSC IL-10. (D) IL-10 production by MDSC decreases macrophage IL-6 and TNF- $\alpha$ and increases macrophage NO. (E) Neutralizing antibodies to IL-10 prevent the down-regulation of macrophage IL-6 and IL-12. (F) Exogenous IL-10 decreases MDSC and macrophage TNF- $\alpha$, decreases macrophage IL-6, and enhances macrophage NO. Macrophages or MDSC were cultured with IL-10 or denatured IL-10. (G) Macrophages activate STAT3 in response to IL-10. Macrophages (left) were cultured for $5 \mathrm{~min}$ in the presence or absence of exogenous IL-10 (middle) or with supernatants (media) from MDSCmacrophage cocultures (right), subsequently fixed and permeabilized, and then stained for F4/80, CD11b, and pSTATs. (H) Macrophages and MDSC express the receptors for IL-10 and IL-6, respectively. (A-H) Data are from one of two, 30, three, five, four, three, two, and two experiments, respectively. Statistical significance was determined by (A-E) Tukey's HSD test and (F) the Mann-Whitney test. Different lower case letters above each value indicate that those values are statistically, significantly different; values that share the same lowercase letter are not statistically, significantly different 


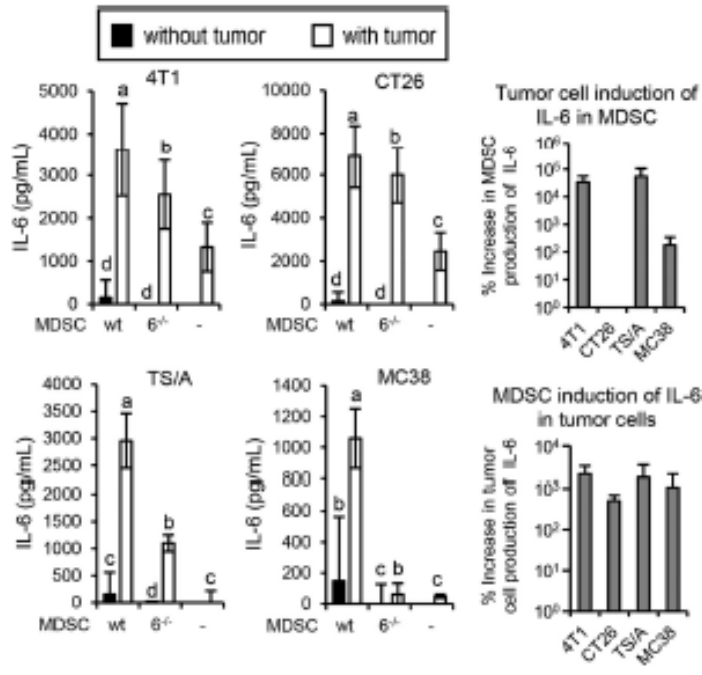

Figure 3. Tumor cells induce MDSC to produce IL-6 and vice versa. WT or IL- $6^{-/-} 4 \mathrm{~T} 1$-induced MDSC were cultured with or without 4T1 CT26, TS/A, or MC38 tumor cells, and the supernatants were assayed for IL-6 by EL.ISA. One of three independent experiments (left four graphs); average percent increase of three independent experiments comparing tumor cells with IL-6 ${ }^{-/-}$and WT MDSC (right two graphs). Statistical significance for the independent experiments was determined by Tukey's HSD test.

levels of IL-6. Cultures of MC38 tumor cells plus IL-6 ${ }^{-/-}$ MDSC produced very low levels of II-6. Increases in IL-6 production in the presence of IL- $6^{-/-}$MDSC indicate that in vitro, MDSC enhanced tumor cell production of IL-6. However, as IL-6 levels in cocultures of WT MDSC plus tumor cells were even higher than IL-6 production in cocultures with IL$6^{-1-}$ MDSC, MDSC may also be induced by tumor cells to synthesize IL-6. Interestingly, MDSC, but not tumor cells, proliferated during the overnight culture (Supplemental Fig. 1C), so the increase in IL-6 in this setting could be a result of higher numbers of MDSC. In contrast, tumor cells did not impact MDSC production of TNF- $\alpha$, IL-12, or IL-10 (Supplemental Fig. 2). These results demonstrate that in vitro, reciprocal cross-talk between MDSC and most tumor cells increases IL-6 production, and there is no cross-talk between MDSC and tumor cells with respect to IL-10, IL-12, or TNF- $\alpha$.

Tumor cells increase macrophage IL-6 and NO and decrease macrophage TNF- $\alpha$

To assess if there is cross-talk between macrophages and tumor cells, 4T1, CT26, TS/A, or MC38 tumor cells were cultured with macrophages and the culture supernatants assayed for IL-6, NO, and TNF- $\alpha$ (Fig. 4). All four tumor lines increased macrophage production of IL-6. Macrophages also increased IL-6 produced by 4T1, CT26, and TS/A tumor cells, as cultures containing tumor cells plus IL- $6^{-/-}$macrophages produced more IL-6 than tumor cells alone. In cocultures of WT

6 Journal or Leukocyte Blology Volume 96, December 2014

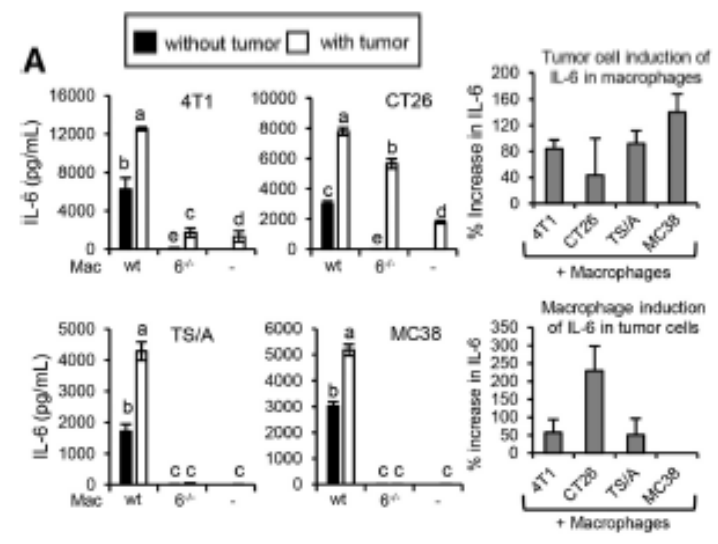

B

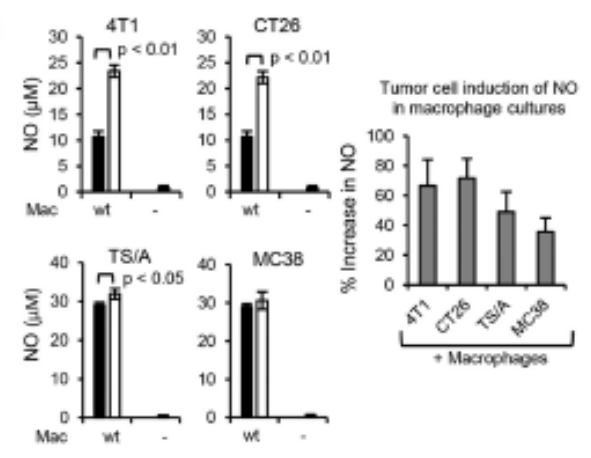

C

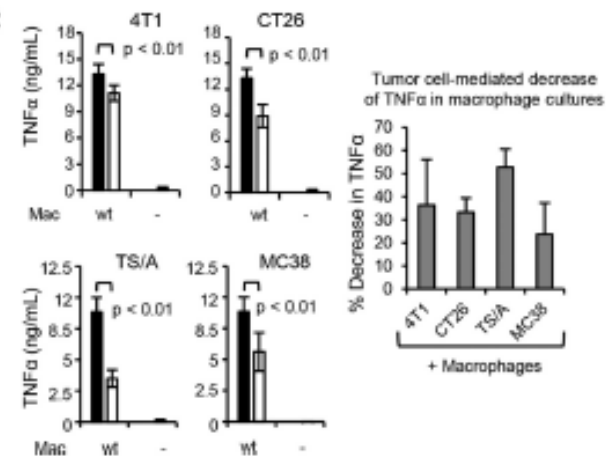

Figure 4. Tumor cells induce macrophages to produce IL-6 and NO but decrease macrophage TNF- $\alpha$. WT or IL $-6^{-/-}$macrophages were cultured with or without 4T1, CT26, TS/A, or MC38 tumor cells, and the supernatants were assayed for (A) IL-6, (B) NO, or (C) TNF- $\alpha$. Representative data (left graphs) from one of four, three, and four independent experiments, respectively. Average percent change of pooled data from all experiments (right graphs). (A) Comparison of tumor cells with IL- $6^{-/-}$and WT macrophages. (B and C) Comparison of tumor cells with WT macrophages. (A-C) Statistical significance was determined by rtest. 
or IL $-6^{-/-}$macrophages with $4 \mathrm{~T} 1$, TS/A, or MC38 tumor cells, macrophages were the dominant producers of IL-6 (Fig. 4A). In contrast, tumor cells were the dominant producers of IL-6 in cultures of macrophages plus CT26 tumor cells, indicating that some tumor cells have a greater response to macrophages. Cross-talk-induced increases in IL-6 ranged from $43 \%$ to $230 \%$. These results indicate that tumor cell production of IL-6 is differentially affected by macrophages and that macrophages produce IL-6 in response to tumor cells.

4T1, CT26, and TS/A cells also increased macrophage production of NO, and increases in NO ranged from $36 \%$ to $72 \%$ (Fig. 4B). In contrast, macrophage production of TNF- $\alpha$ was decreased significantly in the presence of the four tumors, as cultures of macrophages plus tumor cells produced significantly less TNF- $\alpha$ compared with macrophages cultured alone. Tumor cell-mediated decreases in TNF- $\alpha$ ranged from $24 \%$ to 53\% (Fig. 4C). Macrophage production of IL-10 and IL-12 was not affected by tumor cells (Supplemental Fig. 2). Increases in macrophage NO and IL-6 were a result of increased production by individual macrophages, as the macrophages did not proliferate during the overnight culture period (Supplemental Fig. 1C). These results show that macrophages and tumor cells participate in cross-talk with each other, resulting in differential production of proinflammatory mediators, which are characteristic of M1 (NO $\left.{ }^{\mathrm{hi}}\right)$ and M2 (TNF- $\left.\alpha^{\mathrm{low}}\right)$ macrophages.

\section{MDSC prevent most tumor cells from increasing macrophage IL-6}

As MDSC and macrophages are present in the tumor microenvironment, we next tested if MDSC alter cross-talk between tumor cells and macrophages. MDSC, macrophages, and/or tumor cells were cocultured, and IL-6 levels were assessed (Fig. 5A). Cultures containing 4T1, TS/A, or MC38 tumor cells plus MDSC and macrophages produced less IL-6 than cultures without MDSC. MDSC-mediated decreases of IL-6 ranged from $0 \%$ to $37 \%$. In contrast, MDSC did not decrease IL-6 in cultures of macrophages and CT26 tumor cells. These results demonstrate that in the presence of most tumors, MDSC modestly reduce macrophage IL -6 .

\section{MDSC increase macrophage NO in the presence of} tumor cells

To determine if MDSC affect the tumor-driven increase in macrophage NO, tumor cells, macrophages, and MDSC were cocultured (Fig. 5B). Cultures of 4T1 or CT26 tumor cells with macrophages and MDSC contained more NO than cultures without MDSC. MDSC-mediated increases in NO ranged from $0 \%$ to $30 \%$. In contrast, TNF- $\alpha$, IL-10, and IL-12 were not affected by MDSC (Supplemental Fig. 2). These results indicate

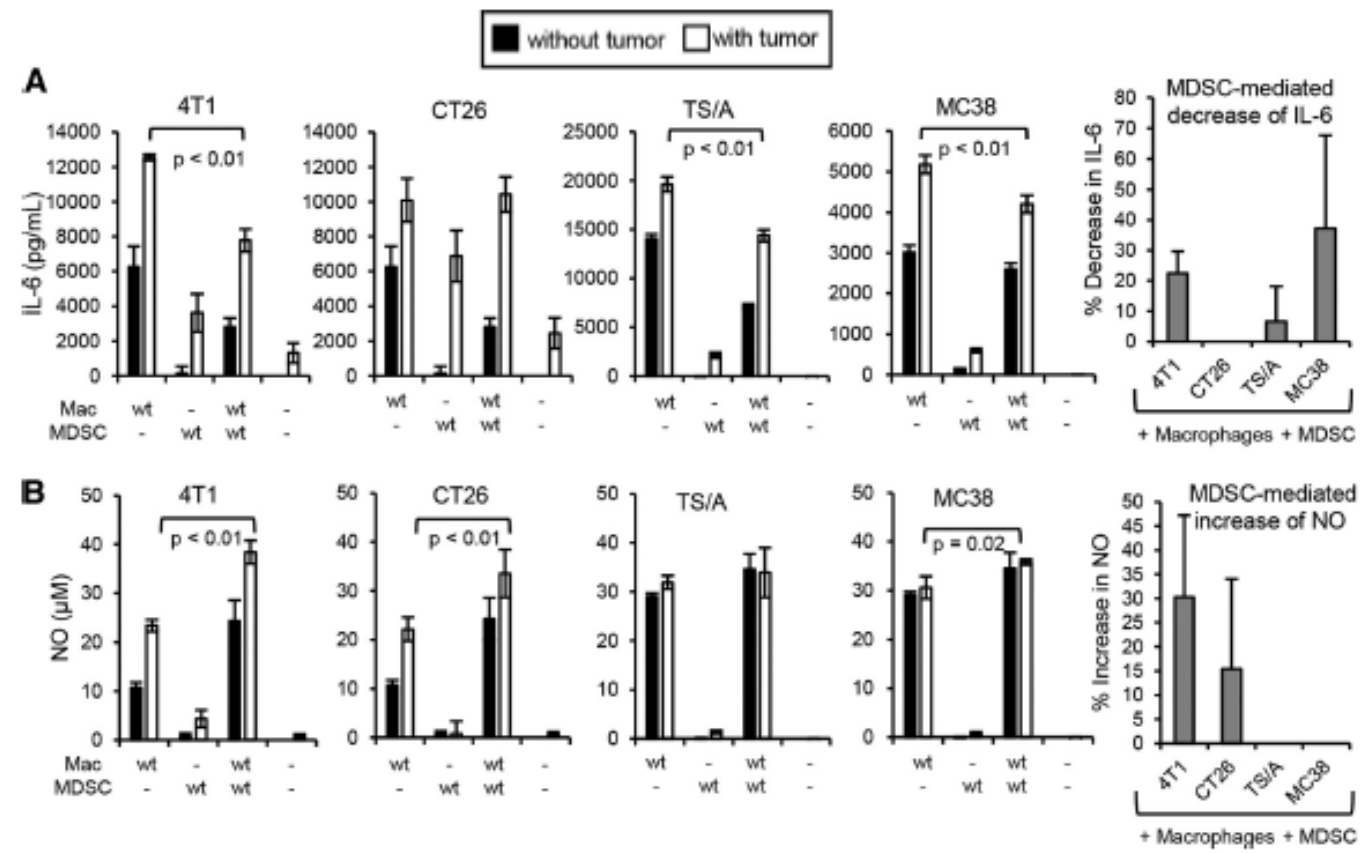

Figure 5. MDSC decrease tumor cell-mediated enhancement of IL-6 and increase tumor cell-mediated enhancement of macrophage NO. WT macrophages were cultured with or without 4T1-induced MDSC and/or 4T1, CT26, TS/A, or MC38 tumor cells. Supernatants were assayed for (A) II-6 and (B) NO. (A and B, left four graphs of each) One of three independent experiments. (Right) Average of the three independent experiments. (A and B) Statistical significance was determined by test. 

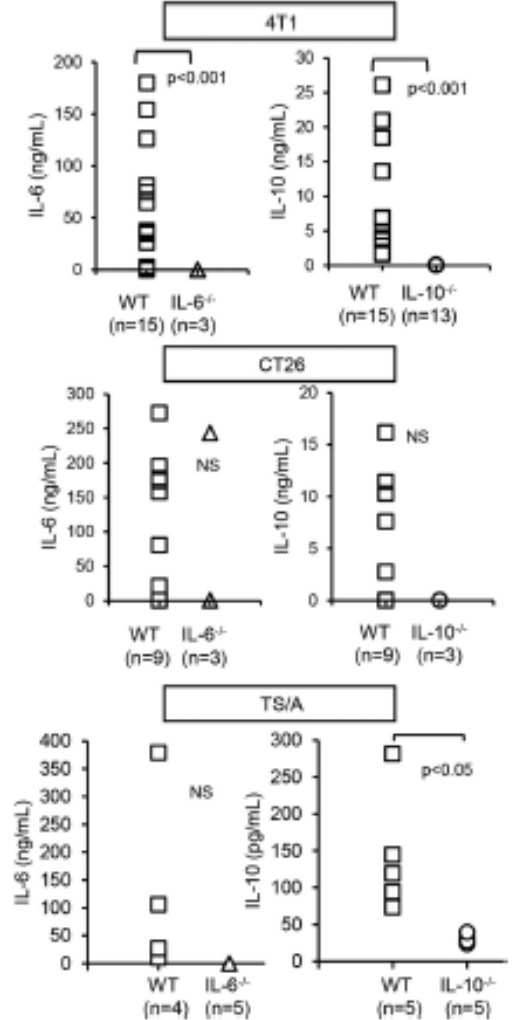

Figure 6. Host cells are the dominant producers of II- 6 and II-10 in the tumor microenvironment. Fight- to $10 \mathrm{~mm}$ diameter $4 \mathrm{~T} 1$, CT26, and TS/A tumors were excised from WT, II. $6^{-/-}$, and II- $-10^{-/-}$BAL.B/c mice, mantally teased into small pieces, and incubated overnight and the supernatants analyzed by EIISA for II-6 and IL-10. Cytokine levels were normalized to $1 \mathrm{~g}$ tumor tissue/mL media. Data are pooled from four independent experiments. Stariscical significance was assessed by mest.

that MDSC alter the dynamic of tumor cell and macrophage cross-talk by enhancing NO production.

Stromal cells are the dominant producers of IL-6 and IL-10 in the tumor microenvironment

Our in vitro findings suggest that tumor-infiltrating cells and not tumor cells are the dominant producers of IL-6 and IL-10. To determine if this in vitro finding occurs in vivo, we harvested 4T1, CT26, and TS/A tumors from WT, IL- $6^{-/-}$, and II- $10^{-/-}$ mice and assayed the tumors for IL-6 and IL-10 (Fig. 6). Tumors in all WT mice contained IL-6 and IL-10, whereas all tumors from II- $10^{-/-}$mice contained very little or no IL- 10 . With the exception of one mouse with CT26 tumor, tumors from IL $-6^{-/-}$ mice did not have IL-6. Isolated tumors did not contain detectable levels of TNF $-\alpha$ or NO (data not shown). These results demonstrate that in vivo in the tumor microenvironment, stromal cells and not tumor cells are the dominant sources of IL-6 and IL-10.

8 Journal of Leukocyte Blology Volume 96, Decernber 2014

\section{DISCUSSION}

Solid tumors include multiple, diverse host cells that contribute to an inflammatory tumor microenvironment and facilitate tumor progression. As macrophages and MDSC are present in most solid tumors, we have examined the interplay of these cells to determine if and how their interactions may influence the intratumor environment. The studies reported here on IL-6, IL-10, $\mathrm{TNF}-\alpha$, and NO, plus our previous reports on IL-12, address some of the most common molecules produced by MDSC and macrophages that contribute to tumor progression. Our findings are summarized in Fig. 7A. Collectively, our results indicate that the levels of IL-6, IL-10, IL-12, TNF- $\alpha$, and NO are modulated by interactions among MDSC, macrophages, and tumor cells. MDSC induce some M2 macrophage characteristics (II--6 $6^{\mathrm{lom}}$ IL

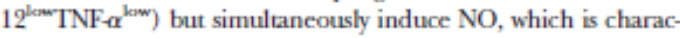
teristic of M1 macrophages. These apparently opposing activities are both regulated by MDSC production of IL-10. Tumor cells
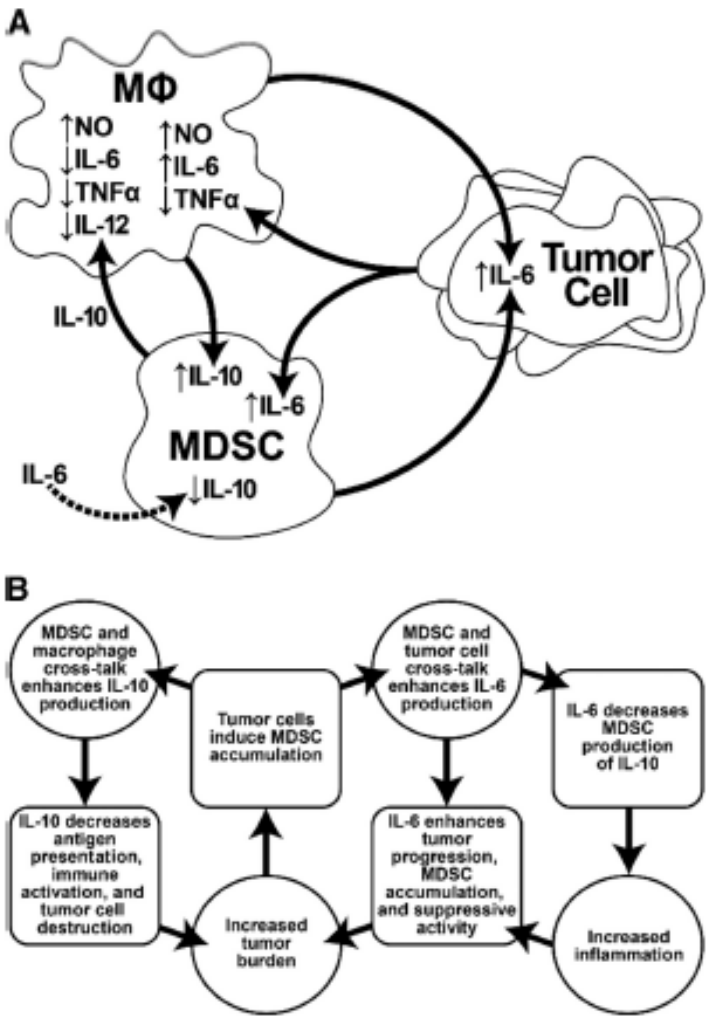

Figure 7. Summary of cross-talk among MDSC, macrophages (MФ), and tumor cells. (A) Cross-talk with respect to IL-10, IL-6, IL-12, TNF- $\alpha$, and NO. Solid arrows indicate direct effects mediated by the cell type or IL-10. Dashed arrow indicates an indirect effect by IL-6. (B) Potential cycle by IL-6, IL-10, and MDSC cross-talk promotes inflammation and tumor progression. 
also regulate macrophage expression of molecules characteristic of M1 (IL- $6^{\text {hi NO }}{ }^{\text {hii }}$ ) and M2 (TNF- $\alpha^{\text {kaw }}$ ) phenotypes, whereas tumor cells and macrophages enhance MDSC production of IL-6 and IL-10, respectively. As stromal cells are the dominant producers in vivo of several of these cytokines, the complex pattern of cross-talk among MDSCs, macrophages, and tumor cells is likely to have profound effects on tumor progression.

$\mathrm{NO}$ is an important effector molecule that is differentially impacted by IL-10 and promotes or inhibits tumor progression depending on the tumor model. NO is produced by eNOS and iNOS, which are up-regulated [26] and down-regulated [27], respectively, by macrophage-produced II-10. Pro- and anti-tumor roles have been attributed to NO/iNOS in multiple tumor systems (Supplemental Table 2). It is likely that the apparent conflicting effects of $\mathrm{NO}$ are a result of many variables, including, but not limited to, the production of NO by different types of cells, the location of the producer cells, neighboring cells that might be altered by the released NO, and the concentration of NO. As a result of the complexity of NO on tumor progression and the presence of multiple cell types in the tumor microenvironment that may participate in cross-talk, elucidating the role of $\mathrm{NO}$ in tumor progression will be challenging.

IL-6 is a pivotal cytokine that promotes tumor progression directly by enhancing tumor cell development, growth, metastasis, vascularization, and inhibiting apoptosis [9-11]. MDSC were reported to be a primary producer of IL-6 in the tumor microenvironment [20]. This observation is consistent with our finding that stromal cells and not tumor cells are the major producer of IL-6 in vivo and that tumor cells drive MDSC IL -6 production. IL-6 also enhances MDSC accumulation and suppressive activity [28-30] and decreases MDSC production of IL-10, an anti-inflammatory cytokine [31]. Therefore, positive feedback between MDSC and tumor cells will potentially maintain chronic inflammation and promote tumor progression through the cycle shown in Fig. 7B.

Pro- and anti-tumor roles have been attributed to II-10. It down-regulates numerous immune-modulatory molecules that are essential for an anti-tumor immune response and is considered an anti-inflammatory cytokine [15, 31]. For example, IL-10 impairs antigen presentation by dendritic cells and macrophages by down-regulating expression of MHC class II, CD80, and CD86. II-10 also decreases production of IFN- $y$ and IL-12, cytokines that are characteristic of and facilitate the development of type I antitumor effector and helper cells, and IL-10 overexpressing tumor cells have increased growth rates in vivo [32]. In cancer patients, secretion of II-10 from basal or squamous cell carcinoma cells prevents in vitro lysis of tumor cells by tumor-infiltrating lymphocytes. In vitro, pretreatment of tumor cells (e.g., melanoma, lymphoma) with II-10 confers resistance to CIL-mediated lysis by decreasing expression of transporter associated with antigen processing 1 and 2 and subsequent surface expression of MHC L II-10 also contributes to tumor progression by enhancing angiogenesis and tumor cell proliferation. As MDSC IL-10 is enhanced by macrophage crosstalk, and II-10 is produced predominantly by umor-infiltrating stromal cells, cross-talk by macrophages and MDSCs is most likely a source of II-10 in the tumor microenvironment.

However, IL-10 has also been linked to enhancing anti-tumor immunity [15]. For example, the reduction in MHC I by IL-10 renders tumor cells more susceptible to NK-mediated killing, and a tumor cell-based glioma vaccine induced more effective antitumor immunity in WT mice than in II $-10^{-/-}$mice. II-10 also activated umor-resident $\mathrm{CD}^{+} \mathrm{T}$ cells directly, facilitated tumor rejection of PDV6 squamous carcinoma [23], and served as an adjuvant in immunotherapy. Treatment of mice with pegylated IL-10, a form of IL-10 that has an increased serum half-life, induced IFN- $\gamma$ and granzyme-B production by tumor-infiltrating $\mathrm{CD}^{+} \mathrm{T}$ cells in a mouse mammary tumor virus tumor model [24]. IL-10 also inhibited tumorigenesis in mice with colon carcinoma and parients with B cell lymphoma [21, 22]. Ablation of IL-10 from $\mathrm{CD}_{4}^{+} \mathrm{T}$ cells enhanced tumor burden in $\mathrm{APC}^{\Delta 468}$ mice [33], whereas $\mathrm{IL}-10^{-1-}$ mice bearing MC38 tumors displayed increased tumor growth, metastasis, MDSC accumulation, and enhanced susceptibility to chemical carcinogenesis [34]. Therefore, as reported in the literature and shown in this report, the role of IL-10 in the promotion of tumor progression is dependent on the tumor model.

STATS is activated by IL-6 and IL-10; however, the two cytokines can result in different biological effects as a result of the complexity of the STAT3 pathway [35]. There are $1.3 \times 10^{6}$ potential binding sites for STAT3 in the mouse genome [36]; however, STAT3 only binds a few thousand sites in a given cell type [37]. STAT3 is a pleiotropic transcription factor that regulates target genes by acting in conjunction with a variety of transcriptional coactivators. The expression of these coactivators is dependent on the cell type and signaling events that occur in a cell's liferime. Many of these coactivators are prebound to STATS target sites (reviewed in ref. [37]). Therefore, a cell's phenotype following STAT3 signaling depends on its previous history with respect to STAT3 activation. The tumor microenvironment is a complex milieu, so differential expression of transcriptional coactivators is likely. As MDSC-macrophage-tumor cell cross-talk involves activation of STAT3 via II-6 and II- 10 , and the relative amounts of these cytokines differ depending on the type of utmor, cross-talk is likely to contribute to the differential effects of IL-10 on tumor progression.

In addition to the cells examined here, other stromal cells also contribute to inflammation within the tumor microenvironment through their cross-talk with MDSC [38, 39]. However, MDSC and macrophages are present at significant levels in most solid tumors, and therefore, their contributions to the inflammatory milieu are likely to be important.

\section{AUTHORSHIP}

D.W.B., K.H.P., P.S., and S.O-R. designed experiments and analyzed data. D.W.B., K.H.P., M.N., K.A.C., and P.S. performed experiments. D.W.B. and S.O-R. wrote the manuscript. All authors approved the manuscript.

\section{ACKNOWLEDGMENTS}

This work was supported by grants from the U.S. National Institutes of Health (RO1CA115880, RO1CA84232). D.W.B. was partially supported by a predoctoral fellowship from the Congressionally Directed Medical Research Programs Breast Can- 


\section{$\mathrm{JLB}$}

cer Program (W81XWH-11-1-0115). We thank Dr. Manfred Kopf for providing breeding stock of BALB/c IL $-6^{-/-}$, Ms. Lisa Burkheimer for her excellent care of our mice, Ms. Virginia Clements for excellent technical support, and Ms. Katelyn Beury for help with the schematic figures.

DISCLOSURES

The authors declare no conflicts of interest

\section{REFERENCES}

1. Shiao, S. L., Ganesan, A. P., Rugo, H. S., Coussens, L. M. (2011) ImDev, 25, 2559-2572

2. Yang, L, Pang Y. Moses, H. L (2010) TCF beta and immune cells an important regulatory axis in the tumor microenvironment and progres. sion. Trends Imwonol 31, 2200-297.

3. Gabrilowich, D. L, Ostrand-Rosenberg, S., Bronte, V. (2012) Coordinatexi reght

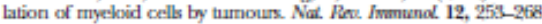

4. Viola, A., Sarukhan, A., Bronte, V., Molon, B. (2012) The pros and cons of chemokines in tumor immunology. Trads /mmunol 33, 496-504

5. Sica, A., Larghi, P., Mancino, A, Rutino, L., Porta, C., Totaro, M. G, Rimoldi, M., Biswats, S. K., Alaverza, P., Mantovani, A. (2008) Macrophtage polarization in tumour progreseion. Savin Caver Hiad. 18, $349-355$

6. Ostrand-Rosenberg, S., Sinha, P. (2009) Mycloidderived suppressor cells linking inflammation and cancer. J. Imownol. 182, 4499-4506.

7. Bunt, S. K., Qeenents, V. K, Harson, E. M, Sinta, P., Otrand-Rosenberg, S (2009) Inflarnmstion entrances myedoidderived sappreseor cell croostalk by

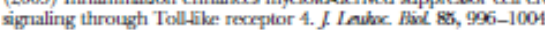

8. Sinha, P., Clements, V. K., Bunt, S. K., Albelda, S. M., Ostrand-Rosenberg, S. (2007) Cross-talk between myeloid-derived suppressor cells and macrophages subverts tumor immunity teward a type 2 response. $f . I m$ wumbl. 179, 977-983.

9. Becker, C., Fantini, M. C., Wirtz, S., Nikolaev, A., Lehr, H. A., Galle, P. R., Rosejohn, S., Neurath, M. F. (2005) II.6 signaling promotes tu mor growth in colorectal cancer. Cell Cycle 4, 217-220.

10. Su, Y. W., Xie, T. X., Sano, D. Myers, J. N. (2011) Il.6 stabilizes Twist and enhances tumor cell motility in head and neck cancer cells through activation of casein kinase 2. PLaS One 6, el9412

11. Santer, F. R., Malinowska, K., Culig, Z, Cavarretta, I. T. (2010) Interleu. kin-6 trans-signalling differentially regulates proliferation, migration, adhesion and traspan expression in human prostate cancer cells. Endoct. Relat. Cancer 17, 241-253.

12. Ralkwill, F. (2006) TNFialpha in promotion and progression of cancer. Cancer Madastasis Rere 25, 409-416.

13. Hussin, S. P., Trivers, G. E., Holseth, L. J, He, P., Staikh, L., Mexharic, L. E. Doja, S, Jang, W., Subkski, J., Shorts, L., Haines, D., Lauksch, V. E, Wiltroul, R. H., Djurickovic, D., Harris, C. C. (2004) Nitric oxisle, a mexliater of inflam mation, suppresses tumorigenesis. Canve Ra. 64, 6849_68553

14. Bronte, V. Zanovello, P. (2005) Regulation of immune responses by larginine metabolism. Nat. Rer Immonol. 5, 641-65.

15. Mocellin, S., Marincola, F. M., Young, H. A. (2005) Interleukin-10 and the immune response against cancer: a counterpoint. $f$ Lewhoc, Biol, 78 , $1043-1051$.

16. Sinha, P., Parker, K. H., Horn, L., Ostrand-Rosenberg, S. (2012) Tumorramed and ind ind

17. Bunt, S. K., Sinha, P., Clements, V. K. Leips, J., Ostrand-Rosenberg. S. (2006) Inflammation induces myeloidderived suppressor cells that facil itate tumor progression. I I mumel. 176, 284 -290.

18. Sintia, P., Qements, V. K, OstrandRosenberg, S. (2005) Rexturtion of myeloidderived sumpressor cells and indurtion of MI mastophanes farilitate the rejoce. tion of established metastatic disesse f I mmunol 174, 656-645.

19. Lippitz, B. E. (2013) Cytokine patierns in patients with cancer, a systematic review. Lance Onol. 14, e218-28.

20. Tsukamoto, H., Nishikata, R., Senju, S., Nishimura, Y. (2013) Myeloidderived suppressor cells attenuate THI development through II-6 production to promote tumor progression. Cance imomenol, Res. 1, 64-76.

21. Berg, D. J., Davidson, N., Kuhn, R., Muller, W., Menon, S., Holland, G. Thompson-Snipes, L., Leach, M. W., Rennick, D. (1996) Enterocolitis and colon cancer in interleukin-10-deficient mice are associated with aberrant cytokine production and $\mathrm{CD} 4(+)$ THI-like responses. $f$. CliniInvest. 98, 1010-1020.

22. Neven, B., Mamessier, E., Bruneau, J., Kaltentach, S, Kotlarz, D., Suarez, F., Masliah-Planchon, J., Billot, K., Canioni, D., Frange, P., Rad-
Casanova, J. L, Blanche, S., Picard, C., Hermine, O, Rieux-Laucat, F., Brousse, N., Davi, F., Raud, V., Klein, C., Nadel, B., Ruemumele, F., Frscher, A. (2013) A Mendelian predisposition to Beell lymphoma caused

23. Emmerich, J., Mumm, J. B., Chan, I. H., LaFace, D., Truong, H., McClanahan, T., Gorman, D. M., Of, M. (2012) IL-10 directly activates and expands tumor-resident CD8( + ) T cells without de nowo infiltration

from secondary lymphoid organs. Cance Rer 72, $3570-3581$.
24. Mumm, J. B., Emmerich, J, Zhang, X., Chan, L., Wu, L., Mauze, S, Blaisdell, S., Basham, B., Dai, J., Grein, J., Shepprard, C., Hong, K., Cut ler, C., Tumer, S., Laliace, D., Keinschek, M., Judo, M., Ayanoglu, G., Langowski, J., Gu, D., Paporello, B., Murphy, E., Sriram, V., Naravula, S, Dessi, B., Medicherla, S., Seghemi, W., McGlanahan, T., CannonCarlson, S., Beebe, A. M., Oft, M. (2011) IL-10 elicits IFNgarmma-dependent tumor immune surveillance. Canca Cell 20, 781-796.

25. Sato, T., Terai, M., Tamura, Y., Alexeev, V., Mastrangelo, M. J., Selvan, S. R. (2011) Interleukin 10 in the tumor microenvironment: a target for anticancer immunotherapy. Immonol. Ra. $51,170-182$

26. Jacobs, F., Chaussabel, D., Truyens, C, Leclerq, V., Carlier, Y., Goldman, M., Vray, B. (1998) IL-10 up-regulates nitric oxide (NO) synthesis by lipopolysaccharide (L.SS)-artivated macrophages improved control of

27. Qusimi, P., Ming-Lum, A, Ghanipour, A., Ong, C.J., Cox, M. E., Ihle, J., Cacalano, N., Yoshimura, A., Mui, A. L. (2006) Divergent mechantisms utilized by socss to mediate interketukn-10 inhibition of tumor necrosis factor alphta and nitric oxide proctuction by macrophages. If. Biol.

28. Ssnith, C., Chang, M. Y., Parker, K. H., Beury, D. W., DuHadaway, J. B. Flick, H. E., Boulden, J, Sutanto-Ward, E., Soler, A. P., Laury-Kleintop, L. D., Mandik-Nayak, L., Meu, R., Ostrand-Rosenberg, S, Prendergast, G. C., Muller, A. J. (2012) IDO is a nodal pathogenic driver of lung cancer and metastasis development. Cance Descou 2, 722-755.

9. Marigo, 1., kxsio, E., Solito, S., Mesa, C., Fertiandez, A., Dolcetti, L., Ugcl, S., Sonda, N., Bicciato, S., Falisi, E, Calabrese, F., Basso, G., Zano vello, P., Cozzi, E., Mandruzzato, S., Bronte, V. (2010) Tumor-induced tolerance and immune suppression depend on the C/EBPtheta tran-

30. Bunt, S. K., Yang, L., Sinha, P., Clements, V. K., Leips, J., OstrandRosenberg, $\mathrm{S}$. (2007) Reduced inflammation in the tumor microenvironment delays the accumulation of myeloid-derived suppre
and limits tumor progression. Cance $R$ r. 67, 10019-10026.

31. Ouyang, W., Rutz, S, Crellin, N. K., Valdez, P. A. Hymowitz, S. C. (2011) Regulation and functions of the II-10 family of cytokines in inflammation and disease. Annu. Reu. Immunel. 29, 71-109.

32. Lee, J. H., Lee, G. T., Woo, S. H., Ha, Y. S., Kwon, S. J., Kim, W. J., Kim, L. Y. (2013) BMP-6 in renal cell carcinoma promotes tumor proliferation through II-10-dependent M2 polarization of tumor-associated macro-

33. phages Cance Res. 73, 3604-3614

Dennis, K. L., Wang, Y., Bhatrer, N. R., Wang, S., Suzadalla, A., Trudeau, E., Roers, A., Weaver, C. T., Lee, J. J., Gilbert, J. A., Chang, E. B.,
Kharaie, K. (2013) Adenomatous polyps are driven by microbe-inst: gated focal inflammation and are controlled by IL-10-producing T cells. Cancer Res. 73, 5905-5913.

34. Tanikawa, T., Wilke, C. M., Kryczek, I., Chen, G. Y., Kao, J., Nunez, G. Zou, W. (2012) Interleukin-10 ablation promotes tumor devclopment,

gro. growth, and metastasis. Cancer Re. 72, 420-429.

Niemand, Cy, Nimmesgern, $A$, Hain, S., Fischer, P., Schaper, F, Rossaint, R., Heinrich, P. C., Muller-Newen, G. (2003) Activation of STAT3 by II-6 and IL-10 in primary human macrophages is differentially modulated by suppressor of cytokine signaling 3. J. Imwonol. 170, 3263-3272. Vallania, F, Schiavone, D., Dewilde, S., Pupo, E., Garbay, S, Calogero,
R., Pontoglio, M., Provero, P., Poli, V. (2009) Genome-wide discovery of functional transcription factor beinding sites by comparative genomics: functional transcription factor binding sites by comparative
the case of Stat3. Ptac. Natl Acad. Sci USA 106, 5117-5122.

37. the case of Stat3. Proc. Natl. Acad. Sac. USA 106, 5117-5122. STATS-mediated anti-inflammatory response: recent developments and future challenges. Brief Funct. Genomies 12, 489-498.

38. Morales, J. K., Saleem, S. J., Martin, R. K., Saunders, B. L., Barnstein, B. O., Faber, T. W., Pullen, N. A., Kolawole, E. M., Brooks, K. B., Norton, S K, seurgill, J., Graham, L., Bear, H. D., Urtain J. F., Jr., Lantz, C. S, Conrsd, D. H., Ryan, J. J. (2014) Myeloid-derived suppressor cells
enhance IgE-mediated mast cell responses. J. Leukac. Biol. 95, 643-650.

39. Saleem, S. J., Martin, R. K., Morales, J. K., Sturgill, J. L., Gibb, D. R. Graham, L., Bear, H. D., Manjili, M. H., Ryan, J. J, Conrad, D. H.
(2012) Cutting edge: mast cells critically augment myeloidederived sur pressor cell activity. $J$. Immanol. 189, 511-515.

KEY WORDS:

cancer · cytokines - tumor microenvironment + IL-6 - IL-10 - nitric oxide 
Appendix 2: IDO is a nodal pathogenic driver of lung cancer and metastasis development 


\section{RESEARCH ARTICLE}

\section{IDO Is a Nodal Pathogenic Driver of Lung Cancer and Metastasis Development}

Courtney Smith ${ }^{1}$. Mee Young Chang ${ }^{1}$. Katherine H. Parker ${ }^{3}$. Daniel W. Beury ${ }^{3}$. James B. DuHadaway ${ }^{1}$. Hollie E. Flick ${ }^{1,4}$. Janette Boulden ${ }^{1}$, Erika Sutanto-Ward ${ }^{1}$. Alejandro Peralta Soler ${ }^{1,7}$.

Lisa D. Laury-Kleintop ${ }^{1}$. Laura Mandik-Nayak ${ }^{1}$. Richard Metz ${ }^{2}$. Suzanne Ostrand-Rosenberg ${ }^{3}$. George C. Prendergast ${ }^{1,5.6}$, and Alexander J. Muller ${ }^{1,6}$ 


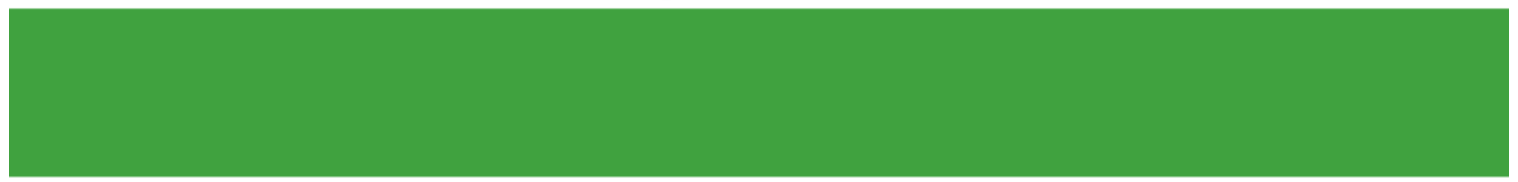

ABSTRACT Indoleamine 2,3-dioxygenase (IDO) enzyme inhibitors have entered clinical trials for cancer treatment based on preclinical studies, indicating that they can defeat immune escape and broadly enhance other therapeutic modalities. However, clear genetic evidence of the impact of IDO on tumorigenesis in physiologic models of primary or metastatic disease is lacking. Investigating the impact of Idol gene disruption in mouse models of oncogenic KRAS-induced lung carcinoma and breast carcinoma-derived pulmonary metastasis, we have found that IDO deficiency resulted in reduced lung tumor burden and improved survival in both models. Micro-computed tomographic (CT) imaging further revealed that the density of the underlying pulmonary blood vessels was significantly reduced in Idol-nullizygous mice. During lung tumor and metastasis outgrowth, interleukin (IL)-6 induction was greatly attenuated in conjunction with the loss of IDO. Biologically, this resulted in a consequential impairment of protumorigenic myeloid-derived suppressor cells (MDSC) as restoration of IL- 6 recovered both MDSC suppressor function and metastas is susceptibility in Idolnullizygous mice. Together, our findings define IDO as a prototypical integrative modifier that bridges inflammation, vascularization, and immune escape to license primary and metastatic tumor outgrowth. SIGNIFICANCE: This study provides preclinical, genetic proof-of-concept that the immunoregulatory enzyme IDO contributes to autochthonous carcinoma progression and to the creation of a metastatic niche. IDO deficiency in vivo negatively impacted both vascularization and IL-6-dependent, MDSCdriven immune escape, establishing IDO as an overarching factor directing the establishment of a protumorigenic environment. Concer Discov; 2(8); 722-35. D2012 AACR.

\section{INTRODUCTION}

Inflammatory tissue microenvironments contribute strongly to tumor progression, but due to the complex multifactoria nature of inflammation, there remains limited understanding of specific pathogenic components that might be targeted to effectively treat cancer (1). In this context, the tryptophancatabolizing enzyme indoleamine 2,3-dioxygenase (IDO) has emerged as an intriguing target implicated in tumoral immune escape $(2,3)$. IDO-inhibitory compounds have entered clinica trials based on evidence of immune-based antitumor responses in a variety of preclinical models of cancer (4-10). Meanwhile, inadvertent IDO targeting may already be providing benefits to patients as illustrated by recent evidence that the clinically approved tyrosine kinase inhibitor imatinib dampens IDO induction as a key mechanism for achieving therapeutic efficacy in the treatment of gastrointestinal stromal tumors (11).

Authors' Affiliations: 'Lankenau Institute for Medical Research-2New Link Genatics Corporation, Wynnewood, Pennsylvania; 'Department of Biological Sciences, University of Maryland Baltimore County, Baltimore, Maryland, ${ }^{4}$ Departmant of Biocherristry. Dreel University Colloge of Maryland; Department of Biechamistry, Dracel Lniversity Colloge of Madicina; Dopartmont of Pathology, Anatomy, and Coll Biology, 'Kimm and PRichfield Laboratory of Dermstopathology, Cincinnsti, Ohig

Note: Supplementary data for this article are availsble at Cancer Discov ary Online (http: //cancerdiscovery.sscrjournals.org/).

C. Smith and M.Y. Chang contributed equally to this study.

Corresponding Authors: Alexander 1. Muller and Gaorge C. Prendergast, Lankenau lnstitute for Medical Research, 100 Lancaster Avenue, Wynnewood, PA 19096. Phone: 484-476-8034 or 8475-Fax: 4B4-476-8533- E-msil: mullera@milhs.org or prendergast@limr.org

doi: 10.115B/2159-8290.CD-12-0014

D2012 American Association for Cancer Resesrch.
Although results with IDO pathway inhibitors are provocative, the conclusions that can be drawn are inherently limited by drug specificity concerns, especially in the absence of independent genetic validation. Addressing this issue, our studies on the impact of 1 dol 1 gene deletion on 7,12-dimethylbenz(a) anthracene/12-O-tetradecanoylphorbol-13-acetate (DMBA/TPA)elicited skin papillomagenesis established that IDO has an integral tumor-promoting role in the context of phorbol ester-elicited inflammation $(12,13)$, but interpretation of these results is tempered by the possibility that the chemical exposures in this model may produce anomalies irrelevant to the majority of spontaneous tumors. The lungs present particularly compelling physiologic context in which to further investigate the role of IDO in tumorigenesis as IDO is known to be highly inducible in this tissue $(14,15)$, and there is an urgent unmet medical need for effective therapeutic options to treat primary lung tumors and metastases. In this report, we investigated the consequences of IDO loss through genetic ablation in the context of well-established, pulmonary models of oncogenic KRAS-induced adenocarcinoma and orthotopic breast carcinoma metastasis. Our findings reveal previously unappreciated roles for IDO in vascularization and in the production of the proinflammatory cytokine interleukin (IL)-6 that in turn dictates the development of protumorigenic, myeloid-derived suppressor cells (MDSC).

\section{RESULTS}

IDO Deficiency Prolongs the Survival of Mice with Sporadic Kras ${ }^{6120}$-Driven Lung Carcinomas

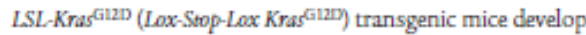
sporadic focal pulmonary adenocarcinomas following intranasal administration of Cre-expressing adenovirus vector 
A

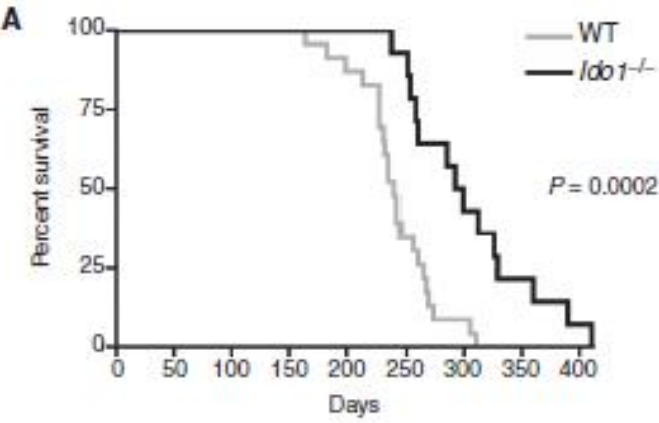

B
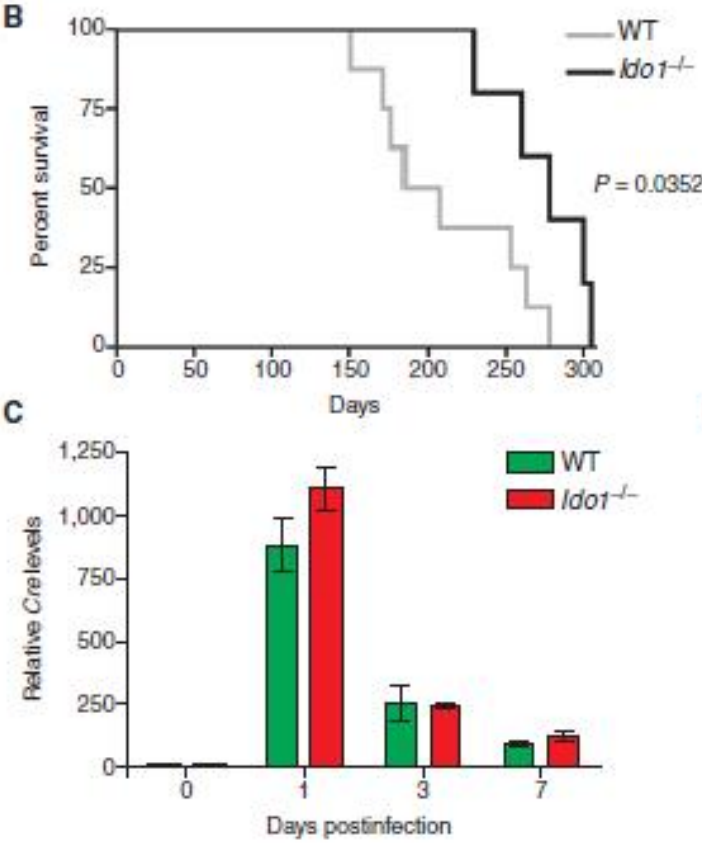

D
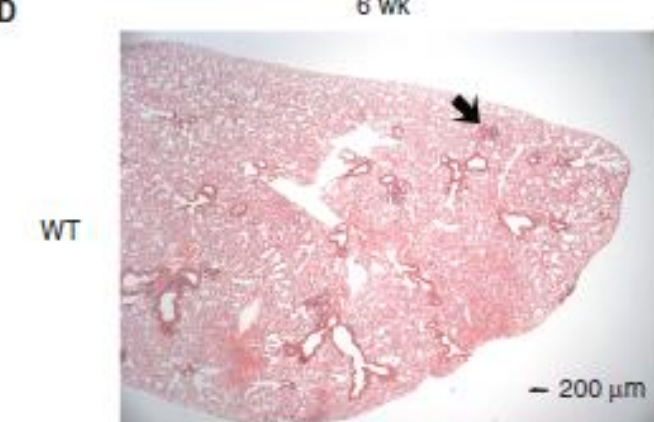

$1001^{-1-}$

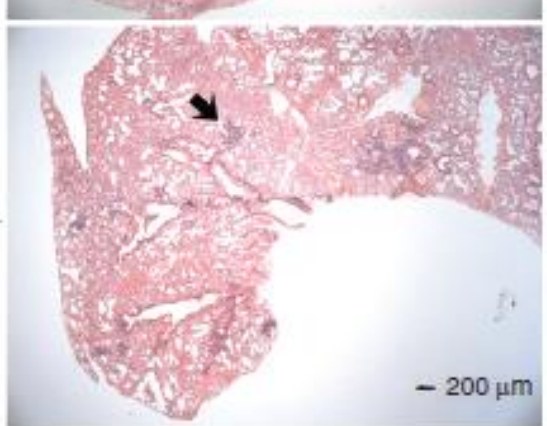

E

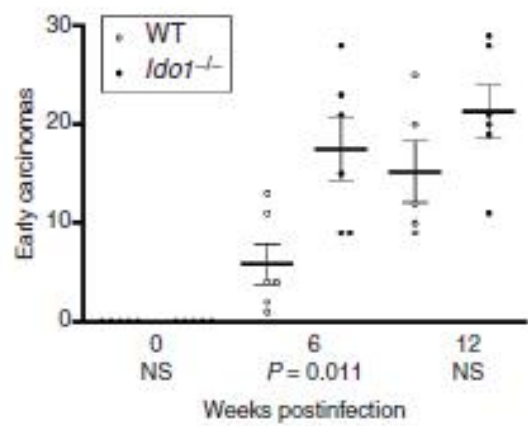

Flgure 1. IDO deficiency extends the survival of mico with KRAS-induced lung odenocarcinomas despite an elevsted number of early lesions.

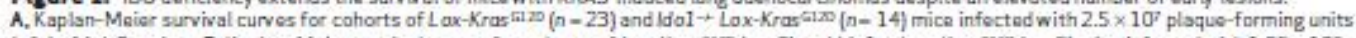

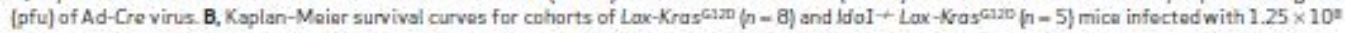
pfu Ad-Cre virus. Significance for both data setsw as assessed by 2-group log-rank test at $P<0.05$. C, total lung DNA prepared from 3 mice per time point was analyzed for the presence of the viral Cre gane by real-time PCR at 0, 1, 3, and7 days postinfection. Relative Cre levals determined from this anslysis aro plotted as means $\$$ SEM. D, representative hematoxylin and eosin (HEE)-stained sections depicting the observod difference in early lesions

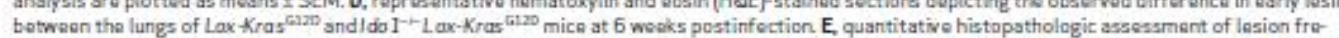

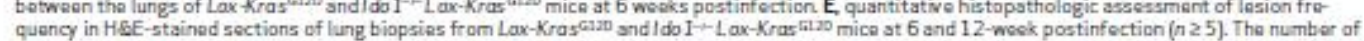
lesions identifiable under low magnification within a dafined region of aach specimen are graphed on the scattar plat with the means \pm SEM. Significance was determined by 2 -tailed Student $t$ test at $P<0.05$. NS, not significant.

(Ad-Cre) to activate the latent oncogenic Kras ${ }^{\mathrm{Cil2m}}$ allele (16). These RAS-induced adenocarcinomas elicit a robust inflammatory response (17) wherein IDO may impart a protumorigenic skew (2). To investigate this hypothesis in an autochthonous lung tumor setting, we introduced Idol'(homoxygous Ido1-null) alleles (18) into the LSL-Krascilid mouse strain. $\mathrm{IdoI}^{+} \mathrm{Lox}-\mathrm{Kras} \mathrm{S}^{\mathrm{G} 2 \mathrm{D}}$ mice displayed significantly increased survival relative to $\operatorname{Lox}-K r a s^{\mathrm{G} i 20}$ mice at 2 different multiplicities of Ad-Cre infection (Fig. 1A and B). Similar levels of Cre were present in the lungs of both strains at $0,1,3$, and 7 days postinfection (Fig. 1C). Unexpectedly, histopathologic examination at 6 weeks revealed that the frequency of early precancerous lesions was actually about 3-fold higher in the $\mathrm{IdOH}^{-1}$ Lox-Kras ${ }^{\mathrm{Gl}} 2 \mathrm{D}$ mice (Fig. ID and E), substantiating 
A

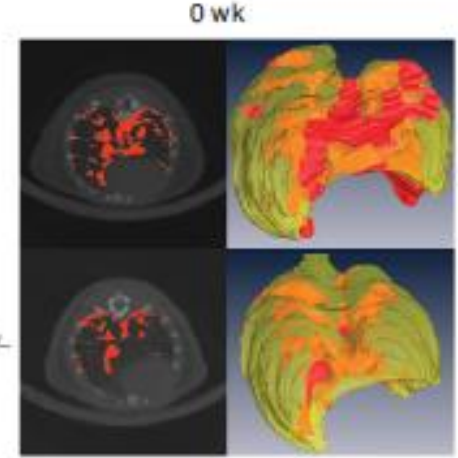

B

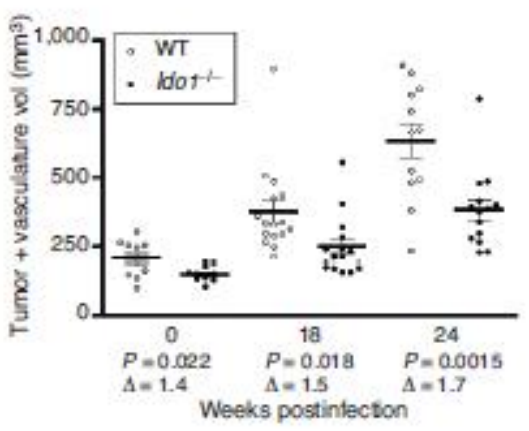

D

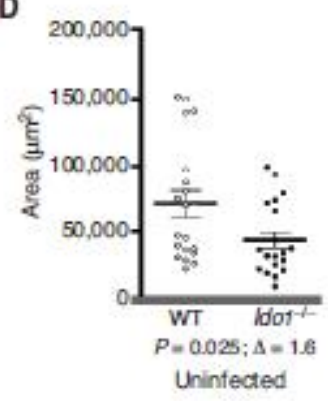

E

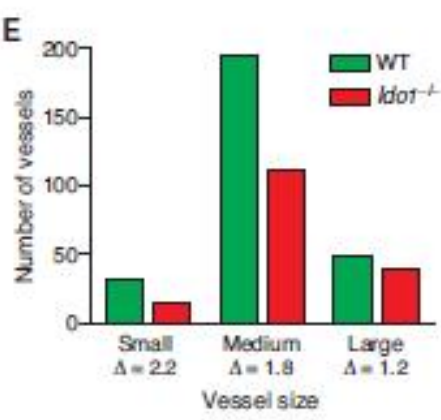

$18 \mathrm{wk}$

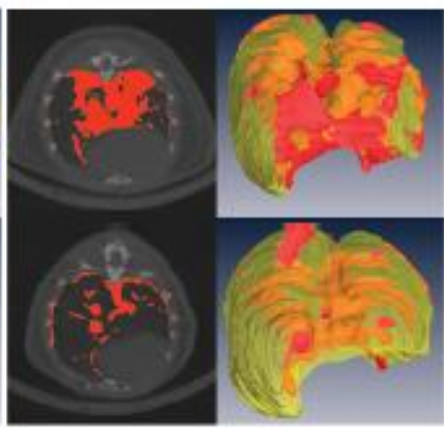

$24 \mathrm{wk}$

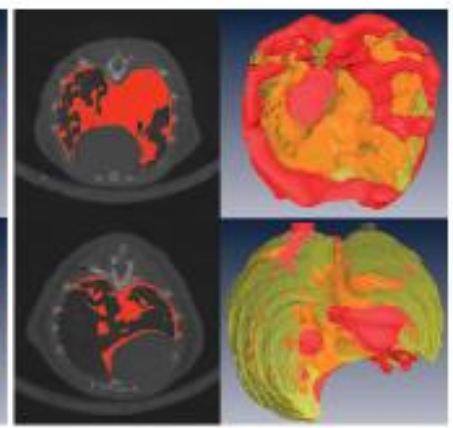

C
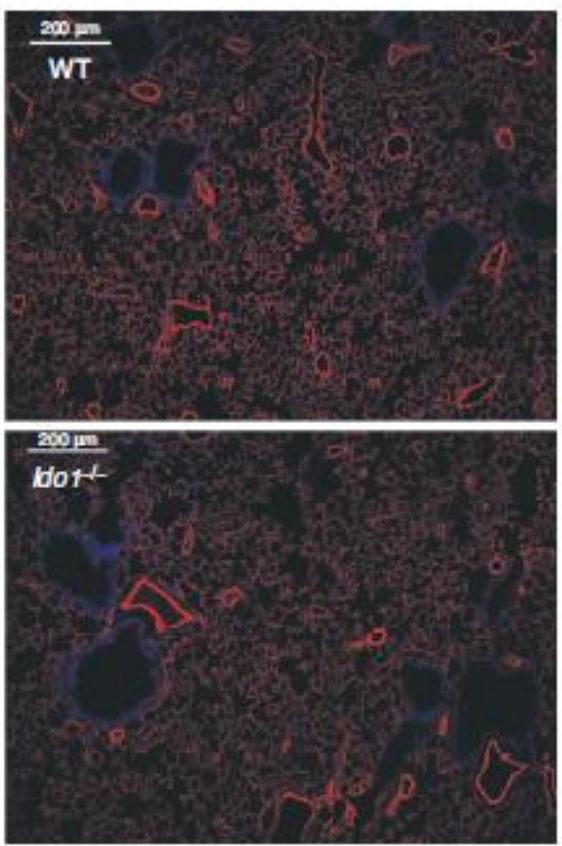

Figure 2. $1 D 0$ deficiency impairs the outgrowth of avert lung adenotarcinomas and reduces narmal pulmonary vascularization. A, representative axial micro-

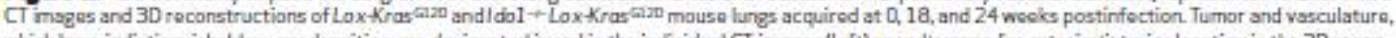
which have indistinguishablex-ray dersities, are designsted in rod in the individusl CT images (left) or redlorange for exteriari interier location in the 3D reconstructions [right] B, volumetric image analysis of tumor and vasculsture conducted on the $3 D$ reconstructions of lurg micro-CT images. The dsta are graphed as a scattar plot with the means \pm SEM (A; fold difference). Significance was determined by 2 -tailed Student t teat at $P<0.05$. C immunofluaroscent staining of blood vessals with artibody to cavoolin 1 (rod) and 4',6-diamidino-2-pharylindale (DAP) staining of nucloi (blue) in representativa lung tissue specimens from BALB/CWT and Ido1 +-mice. D, quantitation of blood vessel areas. The vessel area totals measured within each field are graphed as a scatter plot with the mears \pm SEM [A; fold diffarenco) Significance wss dotermined by 2 -tailed Student $t$ test at $P<0.05$. E, distribution of pulmonary vessols within specified size

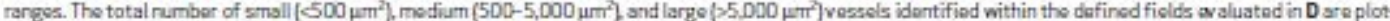
ted on a bar graph ( $\mathrm{A}$ - fold differonca). A lso see Supplementary Fig. S1C for a graph of individual vessel massurements rark ordered scross the antire size range.

that IDO deficiency does not interfere at the stage of Ad-Cre-mediated oncogenic RAS activation required to initiate these tumors (ref. 16; Supplementary Fig. S1A). While early tumorigenesis may be negatively impacted by IDOmediated tryptophan catabolism, as previously proposed (19), this phenomenon was transient with the differential no longer significant by 12 weeks (Fig, 1E).

\section{IDO Deficiency Impairs Tumor Outgrowth and Vascular Development in the Lung}

To assess the impact of IdoI loss on overt lung tumors, noninvasive micro-computed tomographic (CT) scans were con-

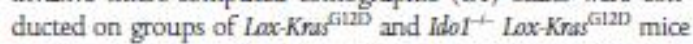
at 18 and 24 weeks following Ad-Cre administration (Fig, 2A). 
A

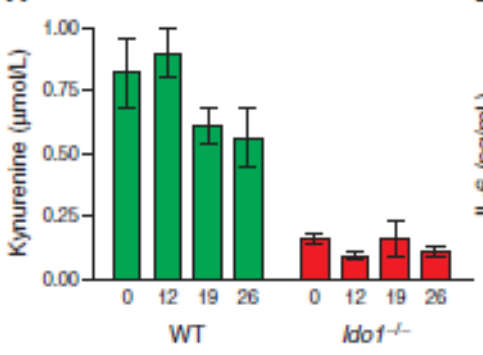

Weeks postinfection

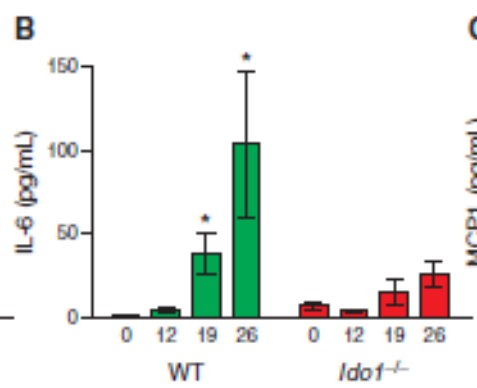

Weeks postinfection c

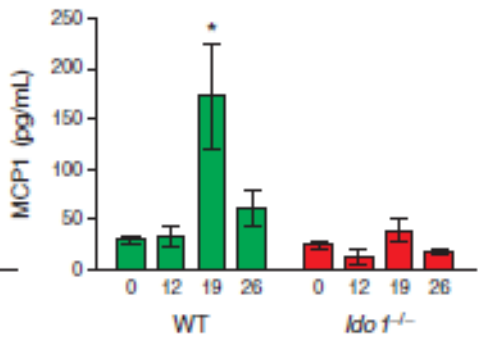

Weeks postinfaction

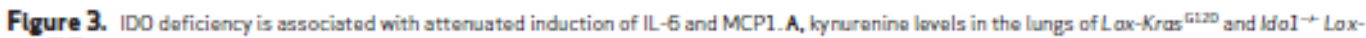

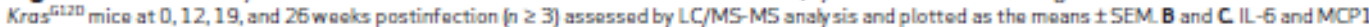

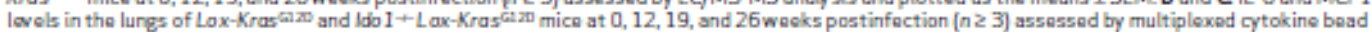
immunoassay-based anslysis and plotted as the mears \pm SEM with significance relative to baseline determined by ane-way ANOVA with Durn test $(*, P<0.05)$.

Semiautomated quantitative image analysis (20) was conducted on 3-dimensional (3D) reconstructions of the thoracic cavity excluding the heart to assess the combined tumor and vasculature volume within this space. Although lung tumor burden did increase progressively in both cohorts, it was significantly

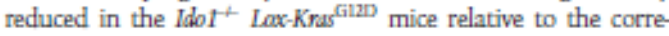
sponding IdoI-competent $L a x-K r a s^{\mathrm{Gl} L \mathrm{D}}$ mice (Fig. $2 \mathrm{~B}$ ). Individual micro-CT scan images paired with 3D reconstructions of total chest space and functional lung volume visually highlight the difference in lung tumor burden between representative Ido $\mathrm{I}^{2}$ Lax-Kras ${ }^{\text {Gil2D }}$ and Krasil2D animals (Fig. 2A; Supplementary Videos). These results indicate that IDO deficiency mitigates overt lung tumor outgrowth, consistent with the increased survival exhibited by these mice.

Micro-CT analysis additionally revealed that the density of normal vasculature in the lungs of uninfected animals was substantially diminished in the $\mathrm{IdoI}^{-6}$ animals (Fig. 2A and B). Intriguingly, the difference in vascular density between IDOdeficient and IDO-competent cohorts was proportionately comparable with the difference in overt lung tumor burden at the 18- and 24-week time points (Supplementary Fig. S1B), suggesting an association between the extent of the underlying basal vasculature and the capacity of the lungs to support tumor formation. Immunofluorescent staining of blood vessels in the lungs confirmed the decrease in pulmonary vascular density in $\mathrm{IdoI}^{-}$animals (Fig. 2C). The area within the lungs occupied by versels was reduced by about 1.6-fold in Ido1 $1^{-}$animals (Fig. 2D), in line with the differential identified by micro-CT data analysis. Further analysis revealed that the reduction in vascular density occurred predominantly at the level of small-to medium-sized vessels, which were nearly twice as abundant in the wild-type (WT) animals, whereas there was little difference in the number of large vessels (Fig. 2E; Supplementary Fig. S1C).

\section{IDO Promotes IL-6 El evation during Lung Tumor Formation}

In the lungs, IDO is highly responsive to pathogen or cytokine exposure $(14,15)$. To determine whether lung tumorigenesis also stimulates IDO, we compared steady-state levels of the tryptophan catabolite kynurenine at various times after Krasilin activation. Although baseline levels of kynurenine in the lungs of uninfected Lox-Kras ${ }^{(12125}$ mice were significantly higher than in their IDO-deficient counterparts (Fig. 3A), these levels remained constant during lung tumorigenesis (Fig. 3A). In contrast, a multiplexed analysis of inflammatory cytokines at 19 and 26 weeks revealed IL-6 to be elevated by about 25 - and 68 -fold, respectively, in lungs from tumor-bearing Lox-Kras ${ }^{\mathrm{G} 12 \mathrm{D}}$ mice but only by about 1- and 3-fold in IdoI ${ }^{+}$Lox-Kras ${ }^{612 D}$ mice (Fig. 3B). This finding was notable given the known tumor-promoting role of IL-6 in this model (21). Although not of the same magnitude, induction of CCL2/MCP1 [chemokine (C-C motif) ligand 2] was likewise attenuated in tumor-bearing Lox-Knas ${ }^{\mathrm{Gi}}$. mice lacking IdoI (Fig. 3C). In contrast, Ido1 loss did not significantly affect the relative levels of IL-10, IFN- $\gamma$, TNF- $\alpha$, or IL-12p70 (data not shown).

\section{IDO Deficiency Impedes the Development of Pulmonary Metastases}

Given the evidence that $I d 1^{-1}$ mice are resistant to the outgrowth of primary lung tumors, we asked whether Ido1' animals might exhibit reduced susceptibility to pulmonary metastasis development as well. This question was investigated by orthotopic engraftment of mice with highly malignant 4T1 breast carcinoma cells, which metastasize efficiently to the lungs. Survival was increased significantly in Idot ${ }^{+}$ hosts compared with WT hosts after challenge with either a 4T1-luciferase-expressing subclone or with parental 4T1 cells, despite an overall shift in the curves (Fig, 4A and B). No difference in primary tumor growth rate was observed (Supplementary Fig. S2A and S2B), but metastatic lung nodules at necropsy were unambiguously less pronounced in $\mathrm{IdoI}^{+}$ mice (Fig. 4C). Noninvasive micro-CT imaging also confirmed a marked reduction in metastatic burden in $\mathrm{Idot}^{-1}$ mice (Fig. 4C), which was quantified by an ex vivo colony-forming assay (ref. 22; Fig. 4D). The metastasis differential was not attributable to reduced intravasation because the same numbers of tumor cells were present in peripheral blood samples from both strains (Fig. 4D). In contrast to lung, 
A

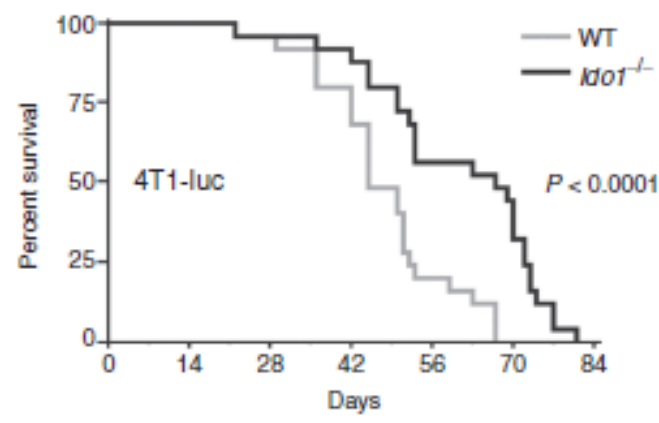

B

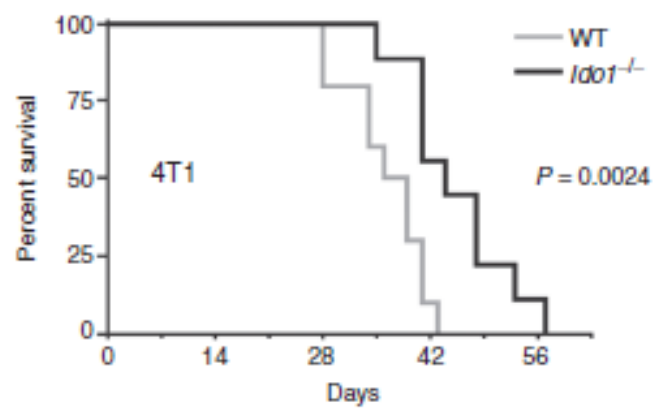

C

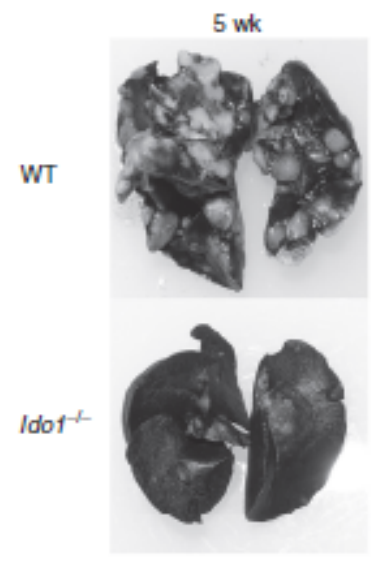

D

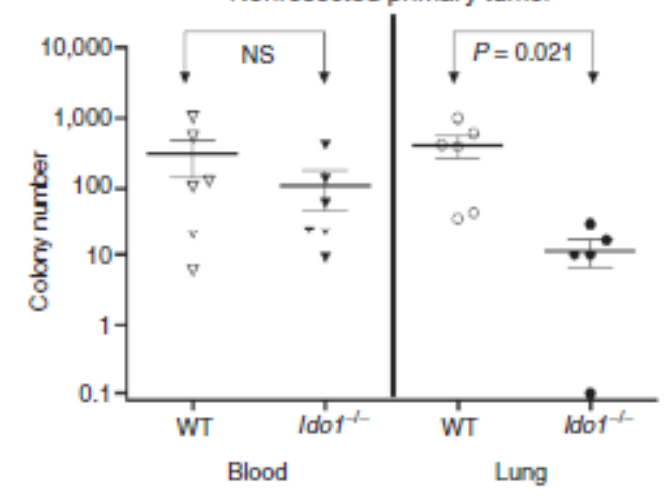

E

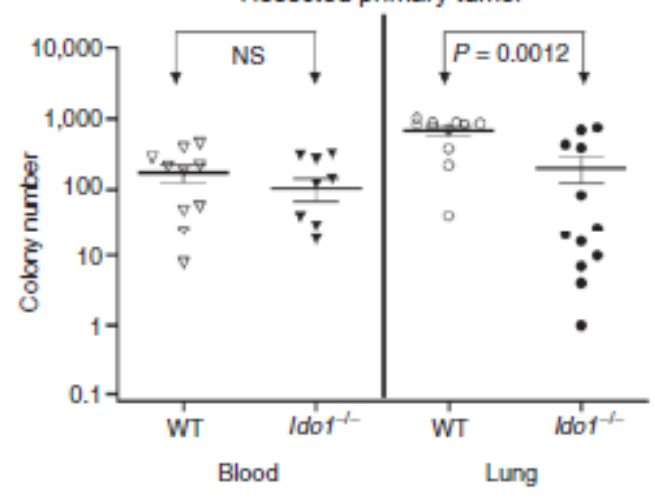

Flgure 4. IDO deficiency delays the devalopment of pulmonary metastases. Kaplan-Maier survival curves for cohorts of WT and ldo1 ${ }^{+-}$mice following orthotopic engraftment of $1 \times 10^{4}(\mathbf{A}) 4 \mathrm{~T} 1$-luc $(n-25)$ or $(\mathbf{B}) 4 \mathrm{~T} 1(\mathrm{n} \geq 9)$ tumor calls. Significance was assessed by 2 -group log-rank test at $P<0.05$. The survival benefit observed in ldo1 $\rightarrow$ mice was independently replicated at University of Maryland Baltimore County. C, staining of lungs with India ink and axisl images from micro-CT scans depicting the difference in pulmonary matastasis burden betwoen WT and ldol ${ }^{*}$ mice at 5 woeks following orthotapic $4 \mathrm{Tl}$ tumor coll engraftment. At 5 woeks following (D) orthotopic angraftement of $4 \mathrm{~T} 1$ cells $(n=6)$ or (E) orthotopic engraftement of $4 \mathrm{~T} 1$ calls and resection

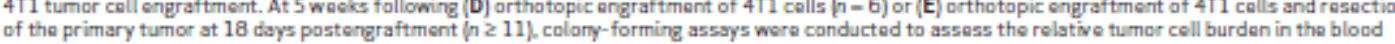
(neat) and lungs (1:1,000). Individusl data points are graphed on a log scale scatter plat with the means \pm SEM and significance assessed by 2-tailed Student $t$ test at $P<0.05$ (NS, not significant). 
A

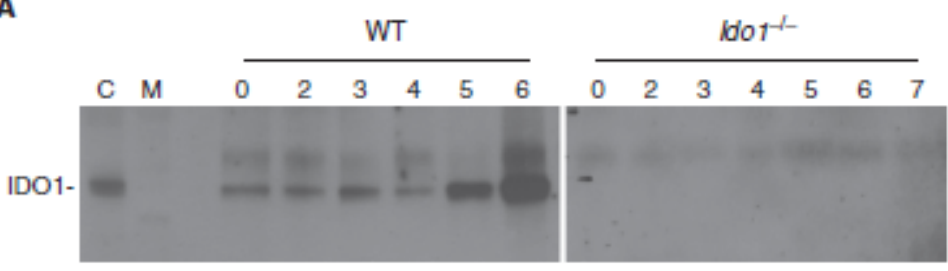

B

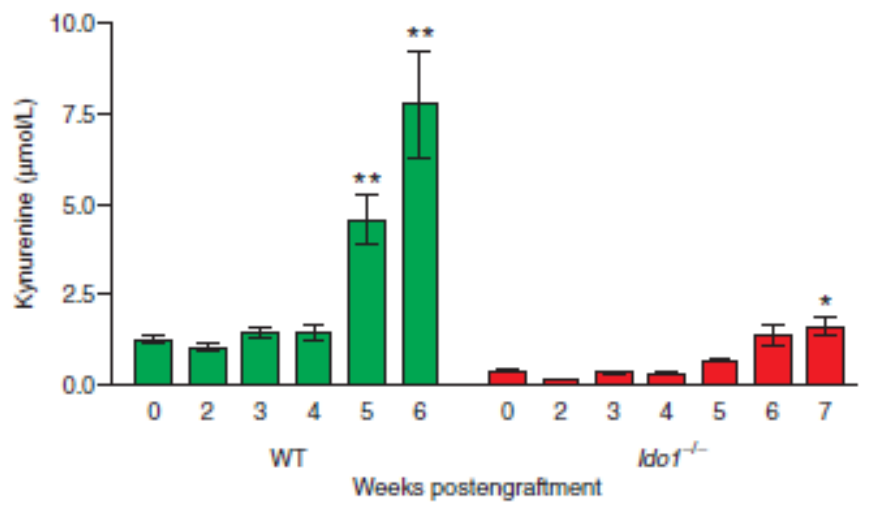

C

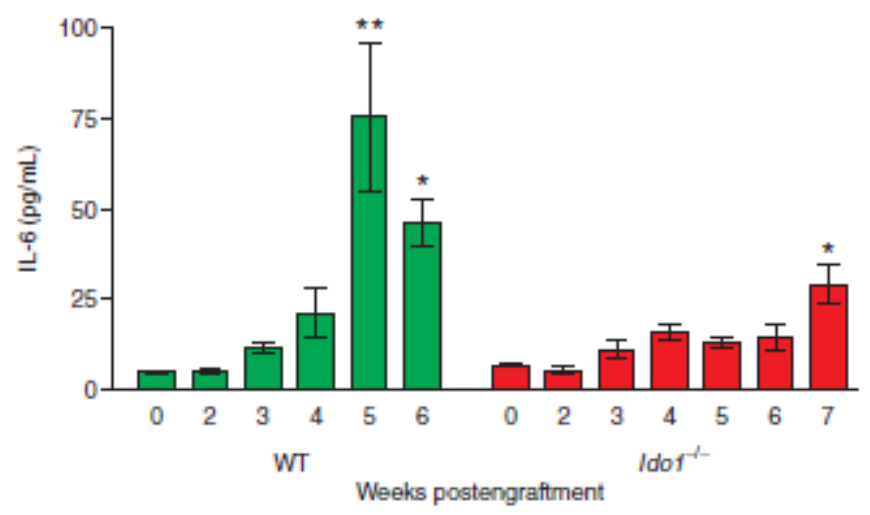

Figure 5. IDO deficiency is associated with attenuated induction of IL- 6 during 4T1 tumor metastasis. A, evaluation of IDO1 protain levels by immunoprecipitation-Western blot analysis of lung tissue lysates from WT and ldol $\rightarrow$ mice following orthotopic engraftment of 4T1 tumor colls at the time points in woeks indicated above each lane. C, apididymis lysate positive control lane; M, molecularwaight marker lane. B, evaluation of kynurenine levels by LC/MS-MS-based anslysis of homogenized lung samples from WT and Ido $\mathrm{I}^{-1}$ mice tumar cells at the time points in weaks indicted for asch lane. Mosns $\pm \operatorname{SEM}(n \geq 6)$ are graphed with sigrificance relative to baseline determined by one-wsy ANOVA with Dunn test $\left(\varphi^{*}, P<0.05 ;^{-*}, P<0.01\right)$. C, IL-6 level determinations from cytokine begd array immunassesy-bosed anglysie of homogenized lung samples from WT and Idol ${ }^{+}$mice following or thotopic engraft ment of $4 \mathrm{Tl}$ tumor cells at the time points in weaks indicted for each lane. Means $\pm 5 E M(n \geq 3)$ are graphed with significance relative to baseline determined by oneway ANOVA with Dunn test [ $P, P<0.05$; $\leftrightarrow, P<0.01$ ) no difference in metastatic burden was observed in liver, although the presence of $4 \mathrm{~T} 1$ cells was also nearly too low to detect in this tissue (Supplementary Fig. S2C). Because excision of the primary tumor can alter immune-based effects on metastasis (23), we evaluated the metastasis burden in resected mice. Ido1 $1^{-}$mice continued to exhibit significant resistance to metastasis development (Fig. 4E), indicating that IDO-mediated support of metastatic development in lung is not dependent on the presence of the primary tumor. We also examined pulmonary VEGF levels but found that these increased comparably in both WT and $\mathrm{Idot}^{-2}$ lungs during metastasis development and were actually some- what higher at baseline in the Ido $1^{-}$lungs (Supplementary Fig. S2D).

\section{IDO Is Activated during Metastatic Lung}

\section{Colonization and Potentiates IL- 6 Induction}

In WT mice, IDO1 protein and kynurenine levels both increased in the lungs during 4T1 metastasis development, particularly at 5 and 6 weeks postengraftment (Fig. $5 \mathrm{~A}$ and B). The principal source of IDO1 expression in this context appears to be the native stroma rather than the engrafted 4T1 tumor cells because no IDO1 protein was detectable in the lungs of Idol ${ }^{-1}$ mice (Fig. 5A), even at 7 weeks postengraftment when 
A
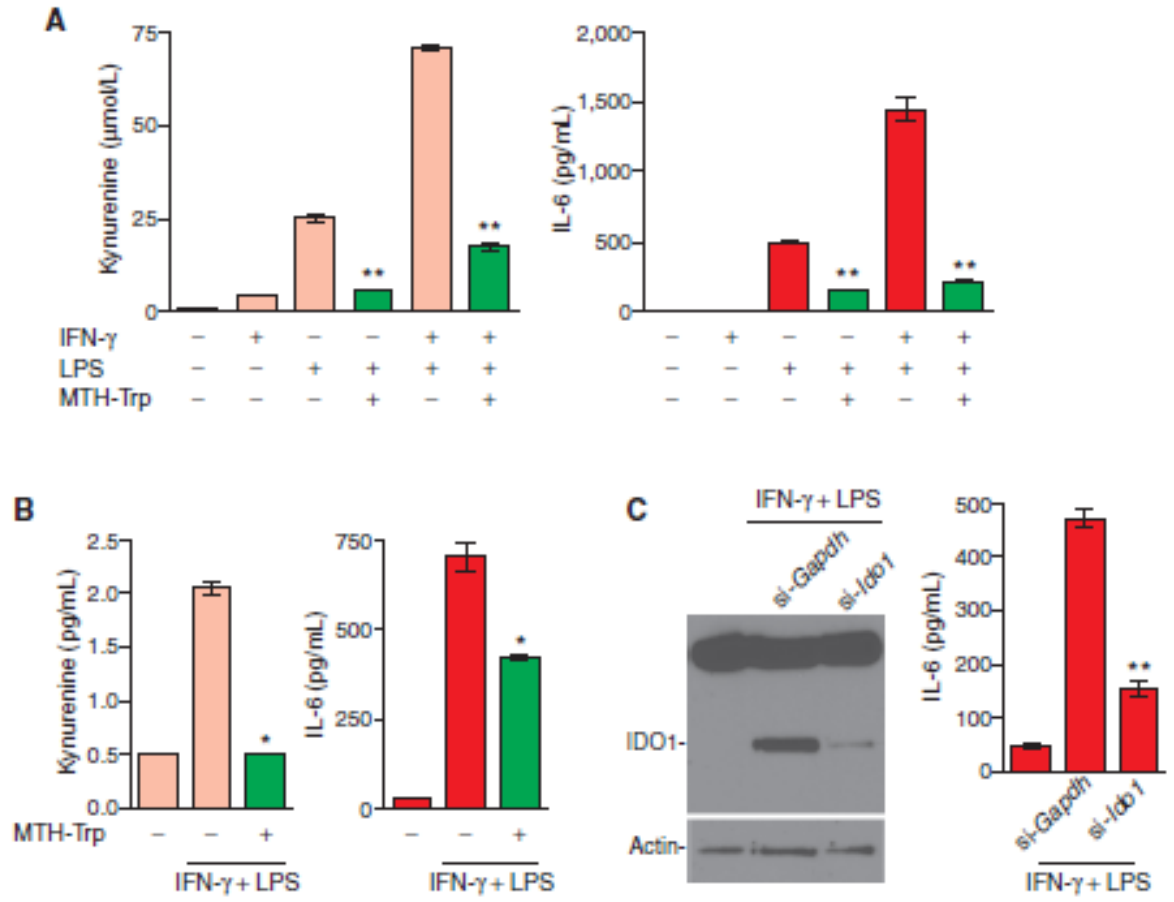

Figure 6. IDO-dependent potentistion of IL-6 production.A, supernatant fram U937 cells stimulated for 24 hours with IFN- $\gamma$ (100 ng/mL) andjor LPS (100 ng/mL) was analyzed for kynurenine and IL-6. Results from triplicate wolls aro platted as the means \pm SEM. Mathylthiohydantoin tryptophan (MTH-Trp, $100 \mu$ molL) was included during induction whare indicated and significance rolstive to the corresponding induced levelwithout MTH-Trp was determined by 2-tailed Student t test (" $\mathrm{mL}$ ) was analyzed for kynurenine and IL- 6 . Results from duplicate wolls are plotted as the means \pm SEM. Mathylthiohydantoin tryptophan (MTH-Trp, $100 \mu m o L L$ ) was included during induction where indicated and significance relative to the corresponding induced level without MTH-Trp was determined

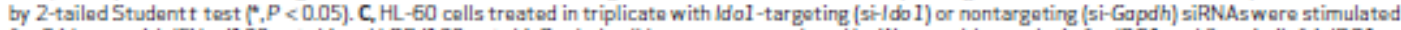
for 24 hours with IFN- $\gamma(100 \mathrm{ng} / \mathrm{mL}$ ) and LPS (100 ng/mL). Pooled coll lysates wore analyz ed by Western blot anslysis for IDO1 and $\beta$ - actin (left). IDD1 inductionwas suppressed by approx imately $89.7 \%$ as as5essed by dersitomatric analysis and normalization to actin. Individual cell supernstants were analyzed for IL- 6 (right). The IL-6 dats aro platted as the mears \pm SEM with the significance of the differenco batwoen specific ldol-targeting versus nontargeting results determined by 2 -tailed Student $t$ test ( $(", P<0.0001)$. Gapdh, glyceraldehyde-3-phosphate dehydrogenase.

the metastatic tumor burden was high. However, a weak but significant increase in kynurenine was observed in the lungs of $\mathrm{LdOI}^{+}$mice (Fig. 5B), suggesting that metastasis development may be associated with induction of an alternative mechanism of kynurenine production, such as IDO2 (24) or TDO2 (tryptophan 2,3-dioxygenase; ref. 25), either in conjunction with or in the absence of IDO1.

As in the Kras-driven primary lung tumor model, IdoI competence in the pulmonary metastatic setting was linked to enhanced elevation of IL-6, with levels increasing up to 15 -fold over baseline in WT animals (Fig. 5C). On the other hand, the IL-6 levels in Ido ${ }^{-}$lungs remained about 2 - to 4 -fold over baseline even when evaluated at an extended time point to account for the differential in tumor burden (Fig. 5C). Thus, like the autochthonous lung tumor stud. ies, results from this lung metastasis model led us to infer a positive regulatory link between IDO and IL-6 production. Direct interrogation of this hypothesis was carried out in a cell-based assay with known IDO inducers. Lipopolysaccharide (LPS) induced both IDO activity and IL-6 production in monocytic U937 cells whereas IFN- $\gamma$ on its own elicited little response but greatly elevated the level of IDO activity in combination with LPS that was mirrored by a comparable enhancement of IL-6 production (Fig. 6A). In both instances, inclusion of the competitive IDO-inhibitory compound MTH-tryptophan (8) significantly suppressed the observed increases in IDO activity as well as IL-6 production (Fig. 6A). MTH-tryptophan-mediated suppression of IL-6 induction was confirmed in a second monocytic cell line HL-60 (Fig. 6B). Likewise, siRNA-mediated interference with IdoI gene expression also significantly suppressed IL-6 induction (Fig. 6C). Taken together, these results are consistent with our in vivo findings suggesting that IDO activity can potentiate the elevated production of IL-6.

\section{IDO Drives MDSC Expansion and Immunosuppressive Function}

Studies in Ill ' (IL-1 receptor-nullizygous) mice have shown a crucial role for IL-6 in 4T1 pulmonary metastasis development (26). At the cellular level, IL-1B enhances development 
A

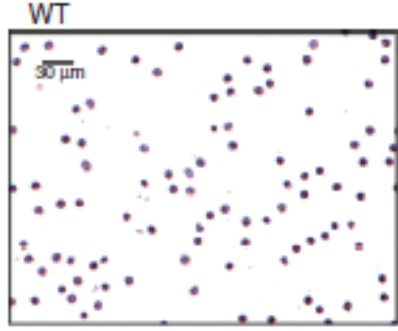

$10 b 1^{1-}$

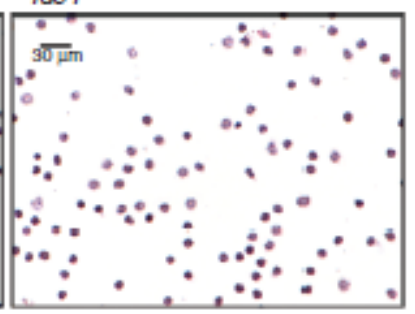

B

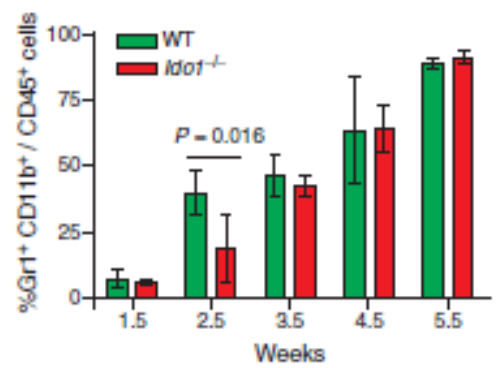

C

$\mathrm{CD}^{+} \mathrm{T}$ cels

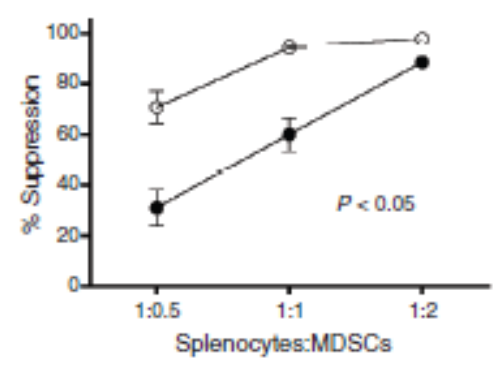

$\mathrm{CDQ}^{+} \mathrm{T}$ cels

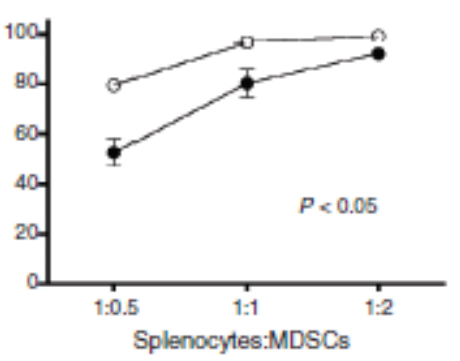

MDSC source

- - WT with 4T1 tumor

$\rightarrow$ ido $t^{-}$with 4T1 tumor

D

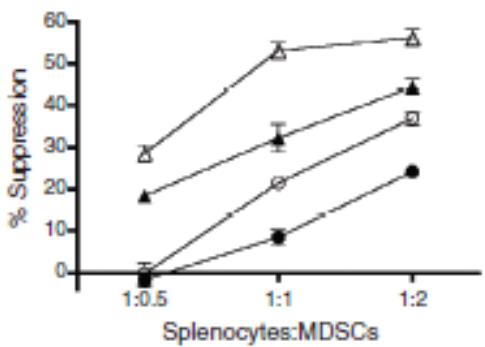

E

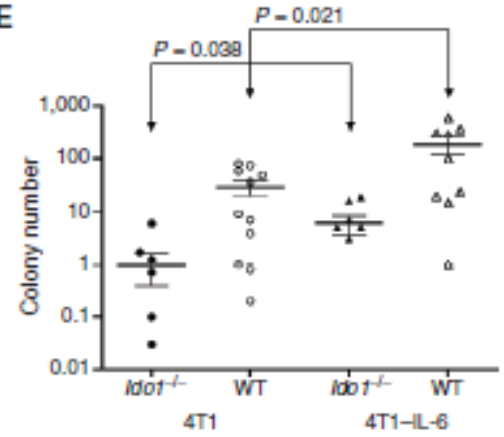

Figure 7. Attenuated MDSC-suppressive activity and metastasis development in IDO-deficient mice is rescued by IL-6. A, comparative microscopic images of hematoxylin and eosin (HEE - stained MDSCs harvested from the blood of WT and Ido 1- mice with primary 4T1 tumors that were not significantly different in size $[12.2 \pm 1.36$ and $11.5 \pm 0.4 \mathrm{~mm}$ in diameter, respectively). B, single-cell suspensions of whole-lung tissues were prepared at the indicated time points following $4 \mathrm{T1}$ engraftment into WT and lodo $1^{+}$mice and evaluated by flow cytomatry for MDSC infiltration by gating on CD 45* colls and anslyzing the Gr 1+CD11 b+ cell populstion. Means \pm SEM are graphed with significance assessed by 2-tailed Student t test at $P$ < 0.05 . C, splenocytes from $\mathrm{CD}^{+} \mathrm{TS} 1$ (left) or $\mathrm{CDB}^{+}$Clone 4 (right) mice were cocultured in triplicate with cognate peptide and increasing proportiora of $4 \mathrm{T1}$-induced peripheral blood MDSCs from WT or Ido1 - mice. T-cell activation was quantified by uptake of Fi-thymidine and graphed as percentage of suppression relative to activation in the absence of MDSC. Significance was assessed by Wilcowon rank test at $P<0.05$. Outcomes are representative of a minimum of 3 independent experiments. D, splenocytes from CDQ' clane 4 transgenic mice ware cocultured with cognate paptide and increasing proportiars of $4 \mathrm{Tl}$ or 4T1-IL-6 tumer-induced MDSC.s from WT or Ido $1^{-1-m i c e}$ for anslysis as in B. Outcomes are representative of 6 independent experiments using TS1, done 4 , or DO11.10 transgenic T cells. E, colony-forming assays to assess the relative turnor cell burden in the lungs conductad 6 weaks following intravenous injection of $4 T 1$ or $4 T 1-1 L-6$ colls into WT and Ido $1^{-1}$ mice. Results are presented on log scale scatter plot with meare $\pm 5 E M$. Significance was assessed by 2 -tailed Student $t$ test at $P<0.05$.

of tumor-promoting MDSCs with IL-6 serving as a critical downstream mediator of this process (26). Because Idol loss attenuated IL-6 induction and metastatic colonization in the lung, we hypothesized that MDSCs may be compromised at some level in tumor-bearing Ido1' mice. MDSCs isolated from WT and $\mathrm{Idot}^{+}$mice did not differ phenotypically
(Fig. 7A; Supplementary Fig. S3A); however, an early delay in the expansion of $\mathrm{Gr} 1^{+} \mathrm{CD} 11 \mathrm{~b}^{+}$cells in $I d o t^{-}$mice, similar to that observed in $\mathrm{ll}^{\prime-1-}$ animals (26), was noted (Fig. 7B). Moreover, circulating MDSCs isolated from Ido/ hosts were functionally impaired in their ability to suppress T cells (Fig. 7C). We did not detect IDO1 protein in $\mathrm{Gr}^{+} \mathrm{CD} 11 \mathrm{~b}^{+}$cells 
obtained from tumor-bearing WT hosts (Supplementary Fig. S3B), consistent with the hypothesis that the observed functional impairment of MDSCs is a non-cell-autonomous effect of IDO deficiency in which IL-6 may act as a key intermediary.

\section{IL-6 Is Critical to IDO-Driven MDSC Activity and Pulmonary Metastasis}

To directly test the ability of IL-6 to functionally restore MDSC-suppressive activity in $\mathrm{IdoI}^{+}$mice, orthotopic tumors were established using 4T1-IL-6 cells (26), a 4T1 cell population engineered to constitutively express IL-6. MDSCs isolated from Ido1 ${ }^{-1}$ mice engrafted with 4T1-IL-6 cells exhibited an elevated T-cell-suppressive activity similar to that of MDSCs isolated from WT hosts engrafted with parental 4T1 cells (Fig. 7D). Further enhancement of MDSC-suppressive activity could be achieved by engrafting 4T1-IL-6 cells into WT mice (Fig. 7D), indicating that the endogenous IL-6 levels stimulated by parental 4T1 tumor cells in WT animals were not fully saturating with regard to promoting MDSC suppressor function.

We next asked whether restoring IL-6 levels could also reverse the metastatic resistance exhibited by $\mathrm{Ido}^{+}$mice. In the orthotopic setting, high levels of IL-6 produced in primary tumors formed by 4T1-IL-6 cells complicated the analysis by impairing the efficiency of pulmonary metastasis [possibly reflecting the recruitment of metastatic cancer cells back to IL-6-expressing primary tumors as documented previously (ref. 27)]. However, as our results in orthotopically engrafted mice had indicated that the IdoI allelic status does not affect $4 \mathrm{~T} 1$ intravasation, we reasoned that a valid assessment of the impact of IDO deficiency on pulmonary metastasis could be made by introducing the metastatic tumor cells directly into the circulation. Accordingly, we confirmed that intravenously engrafted $\mathrm{IdoO}^{+}$mice maintained their resistance to pulmonary metastasis formation, with the apparent mean metastatic tumor burden being 30.4- and 31.6-fold lower in Ido1 $1^{+}$versus WT mice challenged with $4 \mathrm{~T} 1$ and $4 \mathrm{~T} 1$ IL-6 cells, respectively (Fig. 7E). The proportional increase in metastatic burden observed in the 4T1-IL-6 challenged cohorts is also in line with the proposed interpretation of the MDSC functional data that IL-6 is not being produced at saturating levels in the 4T1-challenged WT animals. Because of the significantly higher metastasis burden produced by 4T1-IL-6 cells, comparison of 4T1-IL-6 challenged IdoI' mice to 4T1 challenged WT mice yielded a differential in mean metastatic tumor burden of only 4.8-fold (Fig. 7E). Thus, IL-6 supplementation not only rescued WT levels of MDSC suppressor function in 4T1 tumor-challenged $\mathrm{Kdol}^{2}$ mice but also markedly restored their susceptibility to pulmonary metastasis development.

\section{DISCUSSION}

The idea of immune escape as a "hallmark of cancer" $(28,29)$ represents a groundbreaking although still largely untested paradigm within the field of cancer biology. The presumption that tumors exploit IDO activity as a mechanism of immune escape, initially inferred from the pioneering studies on maternal immune tolerance of Munn and colleagues (30), has become increasingly accepted despite a fundamental deficit in genetic support for the role of IDO in tumor devel- opment. This study addresses this gap with direct genetic validation of the importance of IDO in well-established models of lung cancer and metastasis that offers novel insights into the impact of IDO on tumor pathogenesis. Moreover, these findings strongly encourage the prioritization of clini$\mathrm{cal}$ investigations into the use of IDO pathway inhibitors for treating lung adenocarcinomas and pulmonary metastases where more effective modalities are urgently needed.

While IDO activity was not elevated in lung tissue beyond baseline levels during KRAS-driven lung tumor development, the observed reduction in pulmonary vascularization in $\mathrm{IdoI}^{-+}$animals even before initiation of tumorigenesis implied that the loss of steady-state IDO in this context was sufficiently consequential to impact physiologic processes important to tumor outgrowth. Enhanced tumor vascularization has been reported in tumor xenograft models involving exogenous IDO overexpression (31,32), but our study is the first to identify a role for IDO in supporting vascular development under native physiologic conditions. Our findings likewise genetically establish the importance of IDO activity in nontumor cells for supporting pulmonary metastasis. In this manner, IDO activity may influence metastatic dissemination to tissues such as the lung where its expression is particularly robust. This may, however, be less relevant when IDO [or tryptophan 2,3-dioxygenase (ref. 25)] activity is substantially elevated within the tumor cells themselves $(8,33)$, enabling the malignancy to preemptively shape its surroundings through intrinsic tryptophan catabolism. As such, IDO activity that originates from stromal cells of the tumor microenvironment or from the tumor cells themselves may contribute to directing tumor outgrowth.

The positive association between IDO and IL-6 in lung tumorigenesis and metastasis was not necessarily anticipated, given that it runs counter to expectations based on IDO-mediated induction of liver-enriched inhibitory protein (LIP), a negative regulatory isoform of the Il6 gene expression promoting transcription factor $\mathrm{C} / \mathrm{EBP} \beta(24,34)$. The precise regulatory impact of LIP on Il6 expression is not clear cut, however, insofar as other findings have indicated that LIP can interact with NF-KB to induce rather than limit Il6 transcription (35). Our findings are also consistent with evidence that a downstream product of IDO-mediated catabolism, kynurenic acid, can potentiate IL-6 production in the context of inflammation by signaling through the aryl hydrocarbon receptor (36). IL-6 is a pleiotropic cytokine that is widely implicated in supporting neoplastic outgrowth in the context of chronic inflammation (37). Clinically, IL-6 has been established as a marker of early relapse of resected lung tumors (38). Analyses of DNA polymorphisms in the IL-6 promoter region have identified positive correlations between IL-6 inducibility and lung cancer susceptibility in the context of concurrent inflammatory disease (39) as well as micrometastatic disease in patients with high-risk breast cancer (40). Functionally, IL-6 induction has been identified as an essential downstream component of RAS-induced tumorigenesis (41) that is directly linked to lung tumor development in the Lox-Kras ${ }^{\mathrm{ilizb}}$ transgenic mouse model (21). Numerous other studies indicate that IL-6 can also contribute to tumor promotion by supporting angiogenesis and neovascularization of tumors $(42,43)$. Thus, biologically, 
the epidemiologic and functional data for IL-6 are consistent with the tumor-promoting activity that we have ascribed to IDO through mouse genetics.

Tumor responses to IDO-inhibitory compounds require functional host immunity $(5,6,8,9)$, but the mechanisms through which IDO promotes immune escape have yet to be fully delineated. Connecting IL-6 to IDO provides valuable insight in this regard. IL- 6 has previously been identified in the 4T1 metastasis model as critical to the induction of MDSCs, which act as potent inhibitors of antitumor immunity (44). MDSC accumulation is known to be driven by several factors that are produced by tumor cells and the tumor stroma, including the potent inflammatory media-

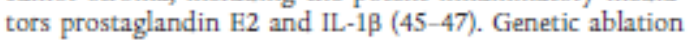
of IL- $1 \beta$ signaling can affect both the early accumulation of MDSCs as well as their immunosuppressive capability (26), and IL-6 has been determined to be a downstream mediator for the effects of IL-1B on MDSC populations in tumor-bearing animals (26). In this context, our findings identify IDO as a key determinant of IL-6-elicited MDSC accumulation and suppressor activity. Interestingly, IL-1 13 may dynamically potentiate the contribution of IDO to IL-6 induction given that $\mathrm{IL}-1 \beta$ can promote the upregulation of IFNGRI (48) that enhances IdoI inducibility in response to IFN- $\gamma$. In contrast, IL-6 may exert a counter-regulatory feedback effect by inducing SOCS3 (suppressor of cytokine signaling 3), which not only attenuates IL-6 signaling (49) but also limits IDO transcription and IDO enzyme stability $(50,51)$. Thus, IDO is well situated to act as a dynamic modifier of inflammatory states in the microenvironment of primary tumors or budding metastases.

Our results deepen the concept that IDO activity profoundly influences the pathogenic character of the tumor microenvironment by identifying the cytokine IL-6 as a crucial IDO effector for establishing "cancer-associated" inflammation. IL-6 is a far-reaching, pleiotropic signaling molecule that can elicit both intrinsic and extrinsic effects on tumor development (i.e., increased malignancy and survival as well as increased angiogenesis and immune escape). The ramifications of our results thus extend beyond the constrained effects that local IDOmediated tryptophan catabolism might exert on the proximal microenvironment, and one would expect the potentiation of IL-6 expression by IDO to affect diverse aspects of tumor development with the relative weighting of each aspect being an important focus of future study. Indeed, further investigations of IDO as a nexus for control of tumorigenic inflammation, vascularization, and immune escape will be invaluable in formulating rational strategies to guide the best application of IDO inhibitors that have entered clinical development.

\section{METHODS}

\section{Transgenic Mouse Strains}

Congenic Idol't mice on C57BL/6 and BALB/c strain backgrounds were provided by A. Mellor (Georgia Health Sciences University, GSHU, Augusta GA), and corresponding control strains were pur chased from Jackson Laboratory. LSL-Kras genic mice on a mixed 1295vJ-C57BL/6 strain background (16) were obtained through the Mouse Models of Hurman Cancer Consortium (NCI-Frederick, Frederick, MD). Administration of Ad-Gre virus to

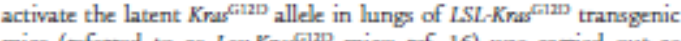
mice (referred to as Lex-Knswald mice, ref. 16) was carried out as described (52). Doubly mutant $L d o I^{+} L S L$-Kras ${ }^{6120}$ mice were generated through breeding of the 2 transgenic strains. Mating pairs of BALB/c and T-cell receptor (TcR) transgenic DO11.10 BAL.B/c mice (I-Ad-restricted, specific for chicken ovalbumin ${ }_{123}$-31) were obtained from The Jackson Laboratory. Mating pairs of TcR transgenic Clone 4 BAL.B/c mice [H-2 $\mathrm{K}^{d}-$ restricted, specific to influenza hemagglutinin (HA) peptide, $515-52 \mathrm{~s}$ ] and TcR transgenic TS1 BALB/c mice (I- $\mathrm{E}^{\mathrm{d}-}$ restricted, specific to HA peptide $110-119$ ) were provided by E. Fuchs (Johns Hopkins, Baltimore, MD). All procedures involving mice were approved by either the Lankenau Institute for Medical Research (LIMR; Wynnewood, PA) or University of Maryland Baltimore County (UMBC) Institutional Animal Care and Use Committer (IACUC).

\section{Micro-CT Scanning}

Three-dimensional micro-CT images were acquired from anesthetized mice using an Impek Micro-CT scanner operated at $40-\mathrm{kV}_{\mathrm{p}}$, $500-\mu \mathrm{A}, 250$-millisecond per frame, 5 frames per view, 360 views, and 1-degree increments per view. Contiguous axial DICOM-formatted images through each mouse thorax, with vowels of dimensions $91 \mu \mathrm{m} \times 91 \mu \mathrm{m} \times 91 \mu \mathrm{m}$ were compiled into 3D format using Amira v5.1 software and normalized to Hounsfield units. Using the seg mentation editor, manual selections of the chest cavity minus the heart were conducted on every other slice followed by interpolation of these selections. Magic wand tool selection was conducted at the threshold range defining air (determined to be between -750 and -350 ) to define the functional lung volume, which was automatically suberacted from the total chest space to identify the wolume representing vasculature and tumors (20).

\section{$4 T 1$ Tumor Cell Metastasis}

Parental 4T1 mouse mammary carcinoma cells and 4T1-derived cell lines expressing luciferase (4T1-luc) or mouse I16 (4T1-IL-6) were maintained as described $(5,22,26)$. Primary turnor growth was monitored by caliper measurements of orthogonal diameters. Tumor volume was calculated using the formula for determining a prolapsed elliptoid $\left[\left(d^{2} \times l\right) / 0.52\right]$, where $d$ is the shorter of the 2 orthogonal measurements. To enhance visualization of metastatic nodules, lungs were insufflated with India ink dye, washed, and bleached in Feketr's solution. The clonogenic assay to assess metastatic burden was conducted as described (22).

\section{Real-time PCR}

Lung DNA was analyzed by Real Time-PCR containing SYBR green PCR master mix (Applied Biosystems) and primers to amplify Cre (5'-GGAGCCGCGCGAGATA-3' and 5'-GCCACCAGCTTGCATGATC-3') and endogenous mouse Cd 81 (5'-TCGCCAAGGATGTGAAGCA-3' and 5'-CATTGTTGGCATCATCATCCA-3'). Assays were conducted in quadruplicate, and relative quantitation of the viral Cre gene present in lung tissue was calculated using the comparative threshold cycle $\left(\mathrm{C}_{\mathrm{T}}\right)$ method (User Bulletin 2, Applied Biosystems) normalizing the target $C_{\tau}$ values to the internal housekeeping gene (CdSI).

\section{Histology}

Tissues were isolated and fixed in $10 \%$ neutral-buffered formalin or $4 \%$ paraformaldehyde, sectioned, and stained for histopathologic analysis with hematoxylin and eosin using standard methods. For

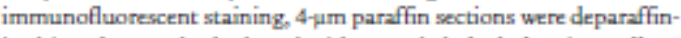
ized in xylene and rehydrated with a graded alcohol series. Following antigen retrieval (vector), sections were washed and placed in $0.1 \%$ Triton for 10 minutes. Tissue was blocked in $40 \mu \mathrm{g} / \mathrm{mL}$ goat anti-mouse IgG-Fab ( $\mathrm{H}+\mathrm{L}$ ) (Jackson ImmunoResearch) followed by 
$10 \%$ normal goat serum (Jackson ImmunoResearch). Rabbit antimouse caveolin-1 (1:200; Cell Signaling) was incubated overnight at $4^{\circ} \mathrm{C}$. Sections were washed and incubated with goat anti-rabbit Cy3 (1:200; Jackson ImmunoResearch). Tissues were mounted using Prolong Gold with DAPI (Invitrogen). To quantitate the blood vessel areas present within defined fields of caveolin-1-stained lung samples, 4 images were acquired per mouse from $5 \mathrm{WT}$ and $5 \mathrm{IdoI}^{-1}$ mice. Vessel boundaries were identified by caveolin-1 staining, and the area of every vessel within each field was determined using AxioVision Release 4.6 software

\section{Immunoprecipitation-Western Blot Analysis}

Immunoprecipitation of IDO1 procein from mouse lung tissur with purified rabbit polyclonal antibody (7) followed by Western blotting-based detection with rat monoclonal antibody (clone mIDO-48; Biolegend) was carried out as described (9).

\section{Flow $C_{y}$ tometry for $C y$ tokine and $C_{e}$ ll Analysis}

Flow cytometric data were acquired on a FACSCanto II or Cyan ADP flow cytometer and analyzed using FACSDIVA (BD Biosciences) or Summit v4.3.02 (Beckman/Coulter) software. Multiplexed cytokine analysis was conducted using the Inflammation Bead Array (BD Biosciences). Lung homogenates were centrifuged and supernatant added to beads in the array according to the manufacturer's instructions. Flow cytometric analysis of MDSCs harvested from digested lung samples or from blood was conducted with the following antibodies as indicated Gr1-PITC, Ly6G-PE, Ly6C-PITC, and CD124 (IL-4R $\alpha$ )-PE (BD Biosciences); CD1 lb-PacB, CD1 15-PE, and F4/B0-PE (BioLegend); Ly6C-PerCP (eBioscience); and arginase and iNOS (BD Transduction Labs). Second step goat anti-mouse IgG-Alexa 647 for arginase and inducible NO synthase (iNOS) was from Invitrogen. Isotype control antibodies were from BD Biosciences.

\section{Kynurenine Assay}

Lungs were homogenized in PBS containing dithiothreitol (DTT) and protease and phosphatase inhibitors (1:3 wt/vol). Deproteinated lysates were analyzed by high-performance liquid chromatography (HPLC) coupled to electrospray ionization liquid chromatography/ tandern mass spectroscopy (LCMS-MS) analysis as described (9).

\section{Cell Culture}

U937 and HL-60 monocytic cell lines (American Type Culture Collection) were expanded for frowen storage after receipt and freshly thawed cells cultured in Dulbecco's Modified Eagle's Media $+10 \%$ FBS were used at early passage for experiments. No additional authentication was conducted by the authors. Twenty-four-hour treatment of cells with L.PS (100 ng/mL; Sigma) and/or IFN- $\gamma(100 \mathrm{ng} / \mathrm{mL}$; R\&ED systems) was carried out in triplicate on $1 \times 10^{4}$ cells per well in a 96-well dish. MTH-Trp (methylthiohydantoin-DL-tryptophan; $100 \mu \mathrm{mol} / \mathrm{L}$; Sigma) was also included at the time of induction as indicated. Kynurenine and IL-6 levels in the supernatant were analyzed as described above. Idol gene "knockdown" studies were conducted with siRNAs (Dharmacon) targeting IdoI (catalog no. E-010337-00) or Gupdb (catalog no. D-001930-01) using the Accell siRNA Delivery System (Dharmacon) as described by the manufacturer. HL-60 cells were plated at $1 \times 10^{4}$ per well in a 96-well dish and cultured with $1 \%$ FBS in the Accell growth media. Twenty-four-hour treatment of cells with LPS and IPN- $\gamma$ was initiated at 48 hours following incubation with siRNA. Western blotting to detect IDOI protein in cell lysates was conducted following standard procedure: using rabbit polyclonal anti-IDO1 (7) and rabbit monoclonal antiB-actin (13E5; Cell Signaling) as a loading control. Detection was carried out with goat anti-rabbit IgG, horseradish peroxidase (HRP)linked secondary antibody (catalog no. 7074; Cell Signaling) using the SuperSignal West Fernto Chemiluminescent substrate (Thermo Scientific)

\section{T-cell Suppression Assay}

MDSC-suppressive activity was measured as previously described (53) using transgenic splenocytes and their cognate peptides in the presence of 25 Gy-irradiated, blood-derived MDSCs from 4TI tumor-bearing mice. $\mathrm{HA}_{5135}, \mathrm{HA}_{110-119}$, and $\mathrm{Ova}_{923-39}$ peptides were synthesized in the Biopolymer Core Facility at the University of Maryland, Baltimore, MD. ELISA duo set mAbs for mIl.6 were from R\&D Systems. Monoclonal antibody VB8.1,8.2-PE was from BD PharMingen.

\section{Disclosure of Potential Conflicts of Interest}

G.C. Prendergast, A.J. Muller, and J.B. DuHadaway declare a potential conflict of interest with regard to IDO due to intellectual property, financial interests, grant support, and consultancy roles with New Link Genetics Corporation, which is engaged in the clinical development of IDO inhibitors for the purpose of treating cancer and other diseases. R. Metz is an employee of New Link Genetics Corporation as Director of Research and has financial and intellectual property interests in the company. No potential conflicts of interest were disclosed by the other authors.

\section{Authors' Contributions}

Conception and design: C. Smith, S. Ostrand-Rosenberg, G.C Prendergast, A.J. Muller

Development of methodology: C. Smith, J.B. DuHadaway, LD. Laury-Kleintop, L. Mandik-Nayak, R. Metz, S. Ostrand-Rosenberg, A.J. Muller

Acquisition of data (provided animals, acquired and managed patients, provided facilities, etc.): C. Smith, M.Y. Chang, K.H.Parker, D.W. Beury, J.B. DuHadaway,J. Boulden, E.Sutanto-Ward, S. Ostrand-Rosenberg

Analysis and interpretation of data (e.g., statistical analysis, biostatistics, computational analysis): C. Smith, M.Y. Chang, K.H. Parker, D.W. Beury, H.E. Flick, A.P. Soler, L.D. Laury-Kleintop, L. Mandik-Nayak, S. Ostrand-Rosenberg, G.C. Prendergast, A.JMuller

Writing, review, and/or revision of the manuscript: C. Smith, D.W. Beury, A.P. Soler, L.D. Laury-Kleintop, L. Mandik-Nayak, R. Metz, S. Ostrand-Rosenberg, G.C. Prendergast, A.J. Muller

Administrative, technical, or material support (i.e., reporting or organizing data, constructing databases): C. Smith, J. Boulden, A.P. Soler, S. Ostrand-Rosenberg

Study supervision: C. Smith, S. Ostrand-Rosenberg, A.J. Muller, G.C. Prendergast

\section{Acknowledgments}

The authors thank Gwen Guillard for tissue sectioning and histol$\mathrm{og} y$ and Lingling Yang for preliminary studies on MDSCs in IDOdeficient mice.

\section{Grant Support}

A.J. Muller is the recipient of grants from Susan G. Kornen for the Cure and the W.W. Smith Foundation. G.C. Prendergast is the recipient of $\mathrm{NIH}$ grants CA109542, CA159337, and CA159315 with additional support from NewLink Genetics Corporation, the SharpeStrumia Foundation, the Lankenau Medical Center Foundation, and the Main Line Health System. S. Ostrand-Rosenberg is the recipient of NIH grants RO1CA115880, RO1CA84232, and RO1GM021248. C. Smith is the recipient of a postdoctoral fellowship through the Department of Defense Breast Cancer Research Program. D. Beury is the recipient of a predoctoral fellowship through the Department of Defense Breast Cancer Research Program. 
Received January 12, 2012; revised May 31, 2012; accepted June 1, 2012; published OnlineFirst July 19, 2012.

\section{REFERENCES}

1. Poek RM Jr, Mohla S, DuBois RN. Inflammation in the genesis and per petuation of cancer. summary and recommendations from a national cancer institute-sponsored meeting. Cancer Res 2005;65:3583-6.

2. Muller AJ, Mandik-Nayak L, Prendergzast GC. Beyond immunosuppressiont reconsidering indoleamine 2,3-dibxygenase as a pathogenic element of chronic inflammation. Immunotherapy 2010;2-293-7.

3. Muller AJ, Scherle PA. Targeting the mechanisms of tumoral immuns tolerance with small-molecule inhibitors. Nat Rev Cancer 2006; 6:613-25.

4. Banerjee T, DuHadarway JB, Gaspari P, Sutanto-Ward E, Munn DH, Mellor AL, et al. A key in vise antitumor mechanism of action of natural product-based brassinins is inhibition of indoleamine 2,3-diaxy. genase. Oncogene 2008;27-2851-7.

5. Hou DY, Muller AJ, Sharma MD, DuHadaway J, Banerjec T, Johnson $\mathrm{M}$, et al. Inhibition of indoleamine 2,3-dioxygenase in dendritic cells by stereoisomers of 1-methyl-tryptophan correlates with antitumor responses. Cancer Res 2007;67:792_B01.

6. Kumar S, Malachowski WP, Duhadaway JB, Lalonde JM, Carroll PJ, aller D, et al. Indoleamine 2,3-dionygenase is the anticancer target for a novel series of potent naphthoquinone-based inhibitors. J Med Chem 2008;51:1706-18.

7. Metz R, Duhadaway JB, Rust S, Munn DH, Muller AJ, Mautino M, et al. Zine protoporphyrin LX stimulates tumor immunity by disrupting the immunosuppressive enzyme indolearnine 2,3-dioxygenase Mol Cancer Ther 2010;9:1864-71.

8. Muller AJ, Duhadaway JB, Donover PS, Sutanto-Ward E, Prendergase GC. Inhibition of indoleamine 2,3-dioxygenase, an immunoregulatory target of the cancer suppression gene Binl, potentiates cancer chemo. therapy. Nat Med 2005;11:312-9.

9. Muller AJ, DuHadaway JB, Jaller D, Curtis P, Metz R, Prendergast GC. Immunotherapeutic suppression of indoleamine 2,3-dioxygenase and tumor growth with ethyl pyruvate. Cancer Res 2010;70:1845-53.

10. Koblish HK, Hansbury MJ, Bowman KJ, Yang G, Neilan C., Haley PJ, et al. Hydroxyamidine inhibitors of indoleamine-2,3-dioxygenase potently suppress systemic tryptophan catabolism and the growth of IDO-expressing tumors. Mol Cancer Ther 2010;9:489-98.

11. Balachandran VP, Cavnar MJ, Zeng S, Bamboat ZM, Ocuin LM, Obaid $\mathrm{H}$, et al. Imatinib potentiates antitumor $\mathrm{T}$ cell responses in gastrointestinal stromal tumor through the inhibition of Ido. Nat Med 2011;17:1094-100

12. Muller AJ, Duhadaway JB, Chang MY, Ramalingam A, Sutanto-W/ard E. Boulden J, et al. Non-hematopoietic expression of IDO is integrally required for inflammatory tumor promotion. Cancer Immunol Immunother 2010;59:1655-63.

13. Muller AJ, Sharma MD, Chandler PR, Duhadanuzy JB, Everhart ME, Johnson BA III, et al. Chronic inflammation that facilitates turnor progression creates local immune suppression by inducing indoleamine 2,3 dioxygenase. Proc Natl Acad Sci U S A 2008; 105:17073_8.

14. Yoshida R, Imanishi J, Oku T, Kishida T, Hayaishi O. Induction of pulmonary indoleamine 2,3-dioxygenase by interferon. Proc Nat: Acad Sci U S A 1981;78:129-32.

15. Yoshida $\mathbb{R}$, Urade $Y$, Tokuda $M$, Hayaishi $O$. Induction of indoleamine 2,3-diaxygenase in mouse lung during virus infection. Proc Natl Acad Sci U S A 1979;76:4084-6.

16. Jackson EL, Willis N, Mercer K, Bronson RT, Crowley D, Montoya R, et al. Analysis of lung tumor initiation and progres. sion using conditional expression of oncogenic K-ras. Genes Dev $2001-15-3243-8$.
17. Ji H, Houghton AM, Mariani TJ, Penera S, Kim CB, Padera R, et al. K-ras activation generates an inflammatory response in lung tumors. Oncogene 2006;25:2105-12.

18. Baban B, Chandler P, McCool D, Marshall B, Munn DH, Mellor AL. Indoleamine 2,3-dioxygenase expnession is restricted to fetal trophoblast giant cells during murine gestation and is maternal genome specific. J Reprod Immunol 2004;61:67-77.

19. Oraki Y, Edelstein MP, Duch DS. Induction of indoleamine 23-dioxygenase: a mecharism of the antitumor activity of interferon gamma. Proc Natl Acad Sai U S A 1988;85:1242-6.

20. Haines BB, Bettano KA, Chenard M, Sevilla RS, Ware C, Angagaw $\mathrm{MH}$, et al. A quantitative volumetric micro-computed tomography method to analyze lung tumors in genetically engineered mouse models. Nesplasia 2009;11-39-47.

21. Ochoz CE, Mirabolfathinejad SG, Ruiz VA, Evans SE, Gagsea M, Evans $\mathrm{CM}$, et al. Interleukin 6, but not $\mathrm{T}$ helper 2 cytokines, promotes lung carcinogenesis. Cancer Prev Res (Phila) 2011;4:51-64.

22. Pulaski BA, Ostrand-Rosenberg S. Mouse $4 \mathrm{TI}$ breast tumor model. In: Coligan JE, Kruisbeek AM, Margulies DH, Shevach EM, Strober W, editors. Curnent protocols in immunology. New York John Wiley \& Sons, Inc.; 2000. p. 20.2.1-16.

23. Ostrand-Rosenberg S, Clements VK, Terabe M, Park JM, Berzofsky JA, Dissanayake SK. Resistance to metastatic disease in STAT6-deficient mice requires hemopoietic and nonhemopoietic cells and is IFN. gamma dependent. J Immunol 2002;169:5796-804.

24. Metz R, Duhadaway JR, Kamassani U, Laury-Kleintop L., Muller AJ, Prendergat GC Novel tryptophan catabolic enzyme IDO2 is the preferred biochemical target of the antitumor indoleamine 2,3-dioxy. genase inhibitory compound D-1-methyl-tryptophan. Cancer Res 2007;67:7082-7.

25. Opitz CA, Litzenburger UM, Sahm F, Ott M, Tritschler I, Trump S, et al. An endogenous tumour-promoting ligand of the human aryl hydrocarbon receptor. Nature 2011;478:197-203.

26. Bunt SK, Yang L, Sinha P, Clements VK, Leips J, Ostrand-Rosenberg S. Reduced inflammation in the tumor microenvironment delays the accumulation of myeloid-derived suppressor cells and limits tumor progression. Cancer Res 2007;67:10019-26.

27. Kim MY, Oskarsson T, Acharyya S, Nguyen DX, Zhang XH, Norton L., et al. Tumor self-seeding by circulating cancer cells. Cell 2009;139:1315-26.

28. Luo J, Solimini NL, Elledge SJ. Principles of cancer therapy: oncogene and non-oncogene addiction. Cell 2009;136:823-37.

29. Prendergat GC. Immune escape as a fundamental trait of cancer. focus on IDO. Oncogene 2008;27-3889-900.

30. Munn DH, Zhou M, Attwood JT, Bondarev I, Conway SJ, Marshall B, et al. Prevention of allogeneic fetal rejection by tryptophan catabo. lism. Science 1998:28 1:1191-93.

31. Nonaka H, Saga Y, Fujiwara H, Akimoto H, Yamada A, Kagawa S, et al. Indoleamine 2,3-dioxyzenase promotes peritoneal dissemination of ovarian cancer through inhibition of natural killer cell function and angiogenesis promotion. Int J Oncol 2011 1;38:113-20.

32. Li Y, Trodget EE, Ghaffari A, Lin X, Kilani RT, Ghahary A. Loca expression of indoleamine 2,3-diourgenase protects engraftment of xenogeneic skin substitute. J Invest Dermatol 2006;126:128-36.

33. Uyttenhove C, Pilotte L, Theate I, Stroohant V, Colau D, Parmentier $\mathrm{N}$, et al. Evidence for a tumoral immune resistance mechanism baeed on tryptophan degradation by indolearnine 2,3-dioxygenase. Nat Med 2003,9:1269-74.

34. Sharma MD, Hou DY, Liu Y, Koni PA, Metz R, Chandler P, et al. Indoleamine 2,3-dioxygenase controls conversion of Foxp3+ Tregs to TH17-like cells in tumor-draining lymph nodes. Blood 2009;113:6102-11

35. Hu HM, Tian Q, Baer M, Spooner CI, Williams SC, Johnson PF, et al. The CIEBP bZIP domain can mediate lipopolysaccharide induction of the proinflammatory cytokines interleukin- 6 and monocyte chemoattractant protein-1.J Biol Chem 2000;275:16373-81. 
36. DiNatale BC, Murray IA, Schroeder JC, Flaweny CA, Lahoti TS, Laurenzana EM, et al. Kynurenic acid is a potent endogenous aryl hydrocarbon receptor ligand that synergistically induces interleukin-6 in the presence of inflammatory signaling. Touicol Sci 2010;115:89-97.

37. Hodge DR, Hurt EM, Farrar WL. The role of IL-6 and STAT3 in inflammation and cancer. Eur J Cancer 2005:41:2502-12.

38. Kita H, Shiraishi $Y$, Watanahe K, Suda K, Ohtsuka K, Koshiishi Y, et al. Does postoperative serum interleukin- 6 influence early recurrence after curative pulmonary nesection of lung cancer? Ann Thorac Car. diovase Surg 2011;17:454-60.

39. Seow A, N $\mathrm{g}$ DP, Choo S, Eng P, Poh WT, Ming T, at al. Joint effect of asthmajatopy and an IL-6 gene polymorphism on lung cancer risk among lifetime non-smoking Chinese women. Carcinogenesis 2006;27:1240-4.

40. De-Michele A, Martin AM, Mick R, Gor P, Wray L, Klein-Cabral M, et al. Interleukin-6-174G- $>$ C polymorphism is associated with improved outcome in high-risk breast cancer. Cancer Res 2003;63-8051-6.

41. Ancrile B, Lim KH, Counter CM. Onoggenic Ras-induced secretion of IL.6 is required for tumorigenesis. Genes Dev 2007;21:1714-9.

42. Angelo LS, Kurarock R. Vascular endochelial growth factor and its rela. tionship to inflammatory mediators. Clin Cancer Res 2007;13-2825-30

43. Grivennikov SI, Karin M. Inflammatory cytokines in cancer: tumour necrosis factor and interleukin 6 take the stape. Ann Rheum Dis 2011;70 Suppl 1:i104-8.

44. Ostrand-Rosenberg S, Sinha P. Myeloid-derived suppressor cells: linking inflammation and cancer. J Immunol 2009;182:4499-506.

45. Bunt SK, Sinha P. Clements VK, Leips J, Ostrand-Rosenberg S Inflammation induces myeloid-derived suppressor cells that facilitate tumor progression.J Immunol 2006;176:284-90.
46. Sinha P, Clements VK, Fulton AM, Ostrand-Rosenberg S. Prostaglandin E2 promotes tumor progression by inducing myelaid-derived suppressor cells. Cancer Res 2007;67:4507-13.

47. Song X, Krelin Y, Dvorkin T, Bjorkdahl O, Segal S, Dinarello CA, et al. CD11 b+/Gr-1+ immature myeloid cells mediate suppression of $\mathrm{T}$ cells in mice bearing tumors of IL-lbeta-secreting cells. J Immunol 2005;175-8200-8.

48. Shirey KA, Jung JY, Maeder GS, Carlin JM. Upregulation of IFN-gamma receptor expression by proinflammatory cytokines influences IDO activation in epithelial cells. J Interferon Cytokine Res 2006:26:53-62.

49. Heinrich PC, Behrmann L, Haan S, Hermanns HM, Muller-Newen G, Schaper F. Principles of interleukin (IL)-6-type cytokine signalling and its regulation. Biochem J 2003;374:1-20.

50. Orabona C, Belladonna MI. Vacca C, Bianchi R, Fallarino F, Volpi C, et al. Cutting edge silencing suppressor of cytokine signaling 3 expression in dendritic cells turns CD28-Ig from immune adjuvant to suppressant. J Immunol 2005;174:6582-6.

51. Orabona C, Pallotta MT, Volpi C, Fallarino F, Vacca C, Bianchi R, et al. SOCS3 drives proteasomal degradation of indoleamine 2,3-dioxygenase (IDO) and antagonizes IDO-dependent tolerogenesis. Proc Natl Acad Sci U S A 2008;105:20828-33.

52. Fasbender $\mathrm{A}$, Lee $\mathrm{JH}$, Walters $\mathrm{KW}$, Moninger TO, Zabner $\mathrm{J}$, Welsh M]. Incorporation of adenovirus in calcium phosphate precipitates enhances gene transfer to airway epitheliz in viro and is vive. J Clin Invest 1998;102:184-93.

53. Sinha P, Clements VK, Ostrand-Rosenberg S. Reduction of myeloid-derived suppressor cells and induction of $\mathrm{Ml}$ macrophages facilitate the nejection of established metastatic disease. J Immunol $2005 ; 1743636-45$. 
Appendix 3: Myeloid-derived suppressor cells: critical cells driving immune suppression in the tumor microenvironment 


\section{CANCER RESEARCH Volume}

128

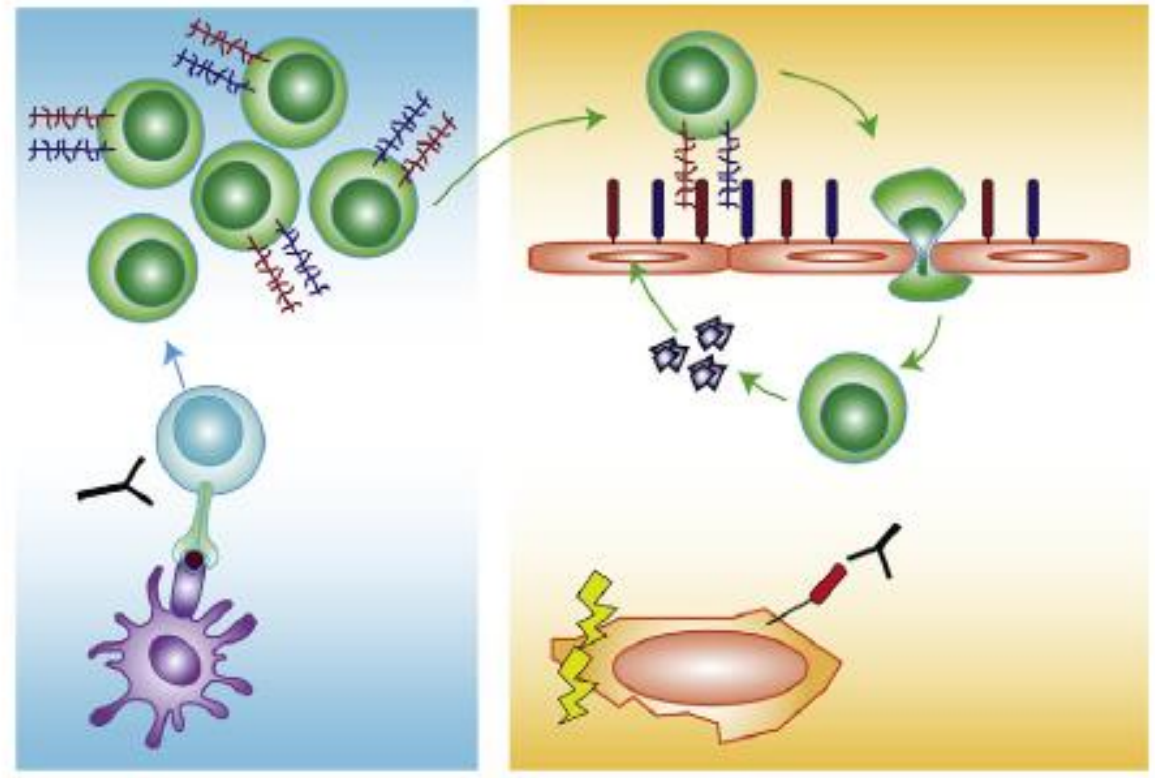

\section{ImMUNOTHERAPY OF CANCER}

Edited by

Xiang-Yang Wang

Paul B. Fisher

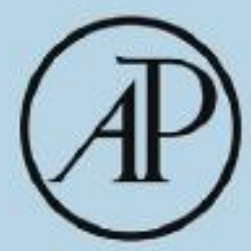




\section{ADVANCES IN \\ CANCER RESEARCH \\ Immunotherapy of Cancer}

\section{Edited by}

\section{XIANG-YANG WANG}

Department of Human and Molecular Genetics, VCU Institute of Molecular Medicine, and VCU Massey Cancer Center, Virginia Commonwealth University School of Medicine, Richmond, Virginia, USA

\section{PAUL B. FISHER}

Department of Human and Molecular Genetics, VCU Institute of Molecular Medicine, and VCU Massey Cancer Center, Virginia Commonwealth University School of Medicine, Richmond, Virginia, USA
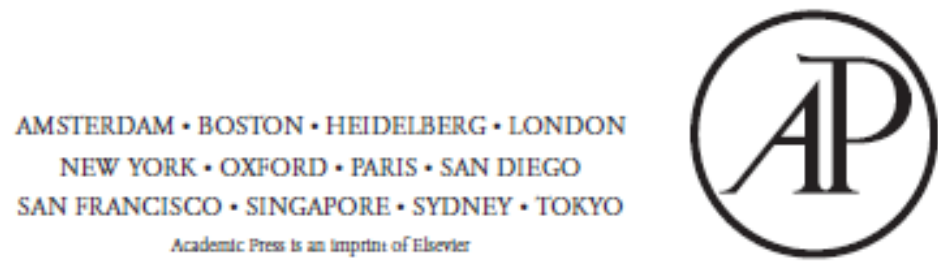
Academic Press is an imprint of Elsevier

225 Wyman Street, Waltham, MA 02451, USA

525 B Street, Suite 1800, San Diego, CA 92101-4495, USA

125 London Wall, London, EC2Y 5AS, UK

The Boulevard, Langford Lane, Kidlington, Oxford OX5 1GB, UK

First edition 2015

(1) 2015 Elsevier Inc. All rights reserved.

No part of this publication may be reproduced or transmitted in any form or by any means, electronic or mechanical, including photocopying, recording, or any information storage and retrieval system, without permission in writing from the publisher. Details on how to seek permission, further information about the Publisher's permissions policies and our arrangements with organizations such as the Copyright Clearance Center and the Copyright Licensing Agency, can be found at our website: www.elsevier.com/permissions.

This book and the individual contributions contained in it are protected under copyright by the Publisher (other than as may be noted herein).

\section{Notices}

Knowledge and best practice in this field are constantly changing. As new research and experience broaden our understanding, changes in research methods, professional practices, or medical treatment may become necessary.

Practitioners and researchers must always rely on their own experience and knowledge in evaluating and using any information, methods, compounds, or experiments described herein. In using such information or methods they should be mindful of their own safety and the safety of others, including parties for whom they have a professional responsibility.

To the fullest extent of the law, neither the Publisher nor the authors, contributors, or editors, assume any liability for any injury and/or damage to persons or property as a matter of products liability, negligence or otherwise, or from any use or operation of any methods, products, instructions, or ideas contained in the material herein.

ISBN: $978-0-12-802316-7$

ISSN: $0065-230 \mathrm{X}$

For information on all Academic Press publications

visit our website at store.elsevier.com

\section{Working together to grow libraries in




\section{Myeloid-Derived Suppressor Cells: Critical Cells Driving Immune Suppression in the Tumor Microenvironment}

Katherine H. Parker ${ }^{1}$, Daniel W. Beury ${ }^{1}$, Suzanne Ostrand-Rosenberg ${ }^{2}$

Department of Biological Sciences, University of Maryland Baltimore County, Baltimore, Maryland, USA

${ }^{2}$ Corresponding author: e-mail address: srosenbe@ umbc.edu

\section{Contents}

1. Myeloid-Derived Suppressor Cell History 96

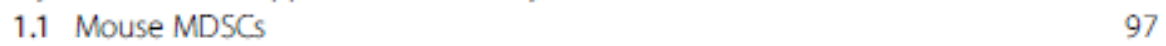

1.2 Human MDSCS 99

2. MDSC Development and Suppressive Functions Are Induced by Inflammation 100

2.1 Vascular Endothelial Growth Factor 101

2.2 Granulocyte-Macrophage Colony-Stimulating Factor and Granulocyte Colony-Stimulating Factor

2.3 Prostaglandin E2 and Cyclooxygenase 2 104

2.4 CCAAT/Enhancer Binding Protein $\beta$ and C/EBP Homologous Protein 104

2.5 Complement Component C5a 105

2.6 S100A8/A9 105

2.7 High-Mobility Group Box $1 \quad 105$

$2.8 \mathrm{IL}-1 \beta$, IL-6, and Indoleamine 2,3-Dioxygenase 106

$\begin{array}{lll}2.9 \quad \text { IL-17 } & 107\end{array}$

3. MDSC Are Regulated by Multiple Molecular Mechanisms 107

3.1 Signal Transducer and Activator of Transcription $1 \quad 109$

3.2 Signal Transducer and Activator of Transcription 3 and $6 \quad 109$

3.3 Nuclear Factor Kappa-Light-Chain-Enhancer 110

3.4 Interferon Regulatory Factor-8 110

3.5 Notch 111

3.6 Hypoxia-Inducible Factor-1 Alpha 111

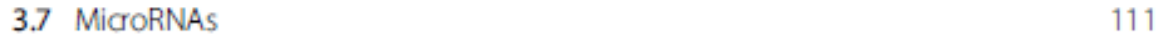

3.8 MDSC Turnover 112

4. MDSCs Utilize a Network of Effector and Signaling Molecules to Modulate the Inflammatory Milieu and Decrease Immune Surveillance 112

4.1 MDSC Depletion of Amino Adids 114

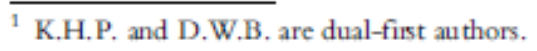


4.2 MDSC Production of NO 115

4.3 MDSC Production of ROS 116

4.4 MDSCs Inhibit T Cell Migration by Downregulating L-and E-Selectins 117

4.5 MDSCs Express Programmed Death-Ligand 1 117

4.6 MDSCs Induce Tregs and Th17 Cells 118

4.7 MDSCs Impair NK Cell-Mediated Cytotoxicity 118

4.8 Cross Talk Between MDSCs, Mađophages, Tumor Cells, and MCs Enhances Inflammation and Promotes MDSC Suppressive Activity 118

5. MDSCs in Noncancer Settings 120

6. Therapeutic Targeting of MDSCs 121

7. Conclusions 126

$\begin{array}{lr}\text { References } & 127\end{array}$

\section{Abstract}

Myeloid-derived suppressor cells (MDSCs) are a heterogeneous population of immature myeloid cells that suppress innate and adaptive immunity. MDSCs are present in many disease settings; however, in cancer, they are a major obstacle for both natural antitumor immunity and immunotherapy. Tumor and host cells in the tumor miqoenvironment (TME) produce a myriad of pro-inflammatory mediators that activate MDSCs and drive their accumulation and suppressive activity. MDSCs utilize a variety of mechanisms to suppress T cell activation, induce other immune-suppressive cell populations, regulate inflammation in the TME, and promote the switching of the immune system to one that tolerates and enhances tumor growth. Because MDSCs are present in most cancer patients and are potent immune-suppressive cells, MDSCs have been the focus of intense research in recent years. This review describes the history and identification of MDSCs, the role of inflammation and intracellular signaling events governing MDSC accumulation and suppressive activity, immune-suppressive mechanisms utilized by MDSCs, and recent therapeutics that target MDSCs to enhance antitumor immunity.

\section{MYELOID-DERIVED SUPPRESSOR CELL HISTORY}

Abnormal myelopoiesis and neutrophilia were observed in cancer patients for many years; however, the role of these pathologies was not appreciated until relatively recently, when myeloid-derived suppressor cells (MDSCs) were identified and associated with immune suppression. Studies from the early and middle 1980 s in tumor-free mice identified a population of so-called natural suppressor cells that inhibited $\mathrm{T}$ cell proliferation and the generation of cytotoxic $\mathrm{T}$ lymphocytes in an antigen and $\mathrm{MHC}-$ independent manner (Strober, 1984). In the 1990s, studies of patients with head and neck cancer described $\mathrm{CD} 34^{+}$-suppressive myeloid cells that had 
the capacity to differentiate into dendritic cells (DCs) (Garrity et al., 1997). Soon after their identification in head and neck cancer patients, similar cells were discovered in patients with various other forms of cancer. These cells prevented the in vivo and in vitro activation of $\mathrm{T}$ cells and were chemoattracted to the tumor microenvironment (TME) by tumor-produced vascular endothelial growth factor (VEGF) (Almand et al., 2001; Young et al., 2001). Mice with transplanted or spontaneous tumors also produced suppressive myeloid cells (Gabrilovich, Velders, Sotomayor, \& Kast, 2001; Melani, Chiodoni, Forni, \& Colombo, 2003), which expressed the granulocyte and macrophage markers Gr1 and CD11b/Mac1, respectively. Their accumulation correlated with tumor-produced granulocyte/ monocyte-colony-stimulating factor (GM-CSF) (Bronte et al., 1999), and they inhibited antigen-specific $\mathrm{CD}^{+} \mathrm{T}$ cell activation in a contactdependent manner (Gabrilovich et al., 2001). Early studies used a variety of terms to identify the cells, including "immature myeloid cells (IMCs)," "immature macrophages (iMacs)," or "myeloid suppressor cells (MSCs)." In 2007, the terminology "myeloid-derived suppressor cells" (MDSCs) was adopted to reflect that the cells are the product of abnormal myelopoiesis (Gabrilovich et al., 2007).

MDSCs differentiate from a common myeloid progenitor cell that also gives rise to normal DCs, monocytes, macrophages, and granulocytes (Fig. 1). Unlike other fully differentiated myeloid cells that are relatively homogeneous, MDSCs are a heterogeneous population of cells since they represent varied stages in myelopoiesis. This heterogeneity is tumor dependent and is most likely spawned from the unique inflammatory milieu released by different tumors. These tumor-released factors, in turn, modulate the recruitment and suppressive potency of tumor-infiltrating MDSCs. The phenotype and functions of MDSCs may also vary with cancer progression since tumor cells evolve and change through immunoediting (Dunn, Bruce, Ikeda, Old, \& Schreiber, 2002). Within this wide array of variation, human and mouse MDSCs have been separated into two major categories: monocytic (MO-MDSC) and granulocytic (PMN-MDSC).

\subsection{Mouse MDSCs}

MDSCs have been identified in the bone marrow, liver, blood, spleen, and tumor of tumor-bearing mice based on their expression of surface markers and their ability to prevent T cell activation. All murine MDSCs express the plasma membrane markers Gr1 and CD11b. The granulocyte marker Gr1 


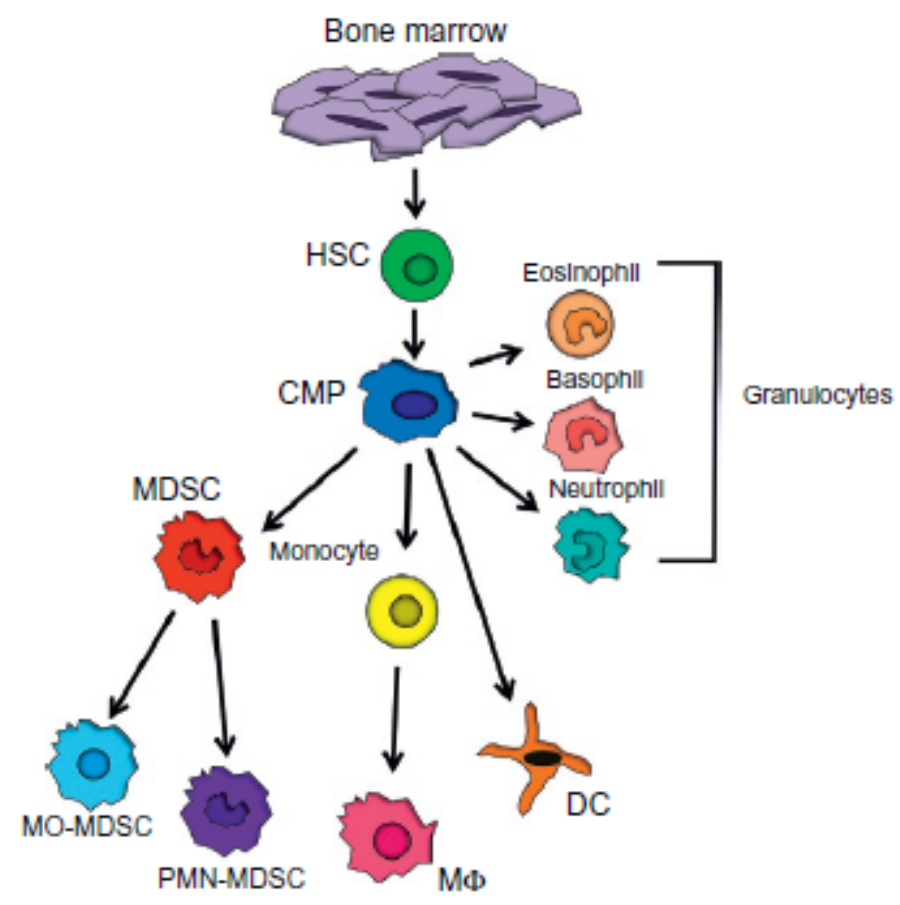

Figure 1 Myeloid cell differentiation under normal and tumor-induced conditions. Myeloid cells originate from bone marrow-derived hematopoietic stem cells (HSCs) that differentiate into common myeloid progenitors (CMPs). During normal myelopoiesis, CMPs differentiate into granulocytes including eosinophils, basophils, and neutrophils, as well as monocytes, macrophages, and dendritic cells. MDSCs also differentiate from CMPs and are categorized as MO-MDSCs or PMN-MDSCs. HSC, hematopoietic stem cell; CMP, common myeloid progenitor; DC, dendritic cell; MФ, macrophage; MO-MDSCs, monocytic myeloid-derived suppressor cells; PMN-MDSCs, polymorphonuclear myeloid-derived suppressor cells.

includes the isoforms Ly6C and Ly6G. The differential expression of these molecules distinguishes MO-MDSCs from PMN-MDSCs. MO-MDSCs are CD $11 \mathrm{~b}^{+}$Ly6C ${ }^{+} \mathrm{Ly}_{6 \mathrm{G}}{ }^{\text {low/-}}$; PMN-MDSCs are CD $11 \mathrm{~b}^{+} \mathrm{Ly}_{6 \mathrm{C}} \mathrm{Ly}_{6 \mathrm{G}}{ }^{+}$. MO-MDSCs are mononuclear and side scatter ${ }^{\text {low }}$, while PMN-MDSCs are polymorphonuclear and side scatter ${ }^{\mathrm{hi}}$. The two subsets use different modes of suppression. PMN-MDSCs utilize reactive oxygen species (ROS) and the enzyme arginase 1 (ARG1), while MO-MDSCs use nitric oxide synthase 2 (NOS2) and ROS. These phenotypes apply to tumorinfiltrating MDSCs, as well as MDSCs residing in the spleen and blood of tumor-bearing mice. Tumor-infiltrating MDSCs are more suppressive than blood or splenic MDSCs on a per cell basis. Tumor-free mice contain cells with the same phenotype $\left(\mathrm{Gr} 1^{+} \mathrm{CD} 11 \mathrm{~b}^{+}\right)$in the blood, spleen, and bone 
marrow; however, they are present at much lower levels compared to tumor-bearing mice (Sinha et al., 2008, 2011).

The markers Gr1 and CD11b as well as the polymorphonuclear morphology of PMN-MDSCs are also characteristics of neutrophils, raising the question of whether MDSCs are different from neutrophils. MDSCs are not neutrophils; however, MDSCs can differentiate into neutrophils. Tumor-associated neutrophils have been categorized as N1, antitumorigenic, and as N2, protumorigenic, with their induction dependent on the presence of IFN $\beta$ or TGF $\beta$, respectively (Fridlender et al., 2009; Jablonska, Leschner, Westphal, Lienenklaus, \& Weiss, 2010). N1 neutrophils are characterized as TNF $\alpha^{\text {hi }}, \mathrm{CCL} 3^{\text {hi }}$, ICAM- $1^{\text {hi }}$, and ARG $1^{\text {low }}$, while N2 neutrophils are high in CCL2, 3, 4, 8, 12, and 17 as well as in CXCL1, 2, 6, and 16 (Sionov, Fridlender, \& Granot, 2014). In contrast to MDSCs, neutrophils do not express CD244 (M-CSF receptor), are more phagocytic than MDSCs, produce lower levels of ROS, have enhanced chemokine secretion, express higher levels of TNF $\alpha$, and most importantly cannot suppress T cell activation (Youn, Collazo, Shalova, Biswas, \& Gabrilovich, 2012).

\subsection{Human MDSCs}

Human MDSCs have been isolated from patients with solid tumors who display elevated MDSC levels that directly correlate with clinical cancer stage and metastatic burden. MDSCs have been found in patients with breast cancer (Alizadeh et al. ,2014; Diaz-Montero et al., 2009), head and neck squamous cell carcinoma (Brandau et al, 2011), nonsmall cell lung cancer (Huang et al., 2013; Srivastava et al., 2008), colon and colorectal cancer (OuYang et al., 2015), renal cell carcinoma (Rodriguez et al., 2009), bladder cancer (Eruslanov et al., 2012), gastrointestinal cancer (Wang et al., 2013), pancreatic adenocarcinoma (Porembka et al., 2012), esophageal cancer (Gabitass, Annels, Stocken, Pandha, \& Middleton, 2011), prostate cancer (Vuk-Pavlović et al., 2010), urothelial tract cancer (Brandau et al., 2011), sarcoma, carcinoid, gall bladder, adrenocortical, thyroid, and hepatocellular carcinoma (Shen, Wang, He, Wang, \& Zheng, 2014). Patients with multiple myeloma and non-Hodgkin's lymphoma also exhibit elevated levels of MDSCs in their blood (Brimnes et al., 2010; Lin et al., 2011).

Since humans lack an analog to Gr1, human MDSCs are characterized by the monocyte/macrophage marker CD11b, the monocyte differentiation antigen CD14, the mature monocyte marker CD15, the myeloid 
lineage markers CD33, and the absence of HLA-DR, which is commonly expressed on myeloid cells (Dumitru, Moses, Trellakis, Lang, \& Brandau, 2012). Similar to murine MDSCs, human MDSCs lack lineage markers characteristic of other hematopoietic-derived cells. Human PMNMDSCs are CD $11 \mathrm{~b}^{+} \mathrm{CD} 14^{-} \mathrm{CD} 15^{+} \mathrm{HLA}-\mathrm{DR}{ }^{\text {low } /-} \mathrm{CD} 33^{+} ; \mathrm{MO}-\mathrm{MDSCs}$ are $\mathrm{CD} 11 \mathrm{~b}^{+} \mathrm{CD} 14^{+} \mathrm{CD} 15^{-} \mathrm{IL} 4 \mathrm{R} \alpha^{+} \mathrm{HLA}_{-} \mathrm{DR}{ }^{\text {low }} \mathrm{CD}_{33}{ }^{+}$(Montero, DiazMontero, Kyriakopoulos, Bronte, \& Mandruzzato, 2012). Since none of the individual markers are unique to MDSCs, definitive identification of MDSCs requires demonstration of immune-suppressive function.

\section{MDSC DEVELOPMENT AND SUPPRESSIVE FUNCTIONS ARE INDUCED BY INFLAMMATION}

Studies evaluating patients on long-term use of nonsteroidal antiinflammatory drugs, epidemiological analyses, and trials involving blockade of inflammatory molecules have demonstrated that inflammation contributes to the onset of cancer (Balkwill \& Mantovani, 2001). Four main sources of inflammation promote carcinogenesis: environmental inflammation, therapy-induced inflammation, tumor-associated inflammation, and chronic inflammation or infection.

Particulates from tobacco smoke are an example of an environmental source of inflammation. They cause chronic obstructive pulmonary disease which is associated with increased lung cancer (Punturieri, Szabo, Croxton, Shapiro, \& Dubinett, 2009). Therapy-induced inflammation occurs following radiation and chemotherapy. It causes necrotic death of cancer cells and tumor stromal cells and initiates an inflammatory response similar to wound-healing (Zong \& Thompson, 2006). Therapy-induced inflammation may enhance presentation of tumor antigens; however, it may also create tumor-promoting inflammation (Zitvogel, Apetoh, Ghiringhelli, \& Kroemer, 2008). Many tumors are inherently inflammatory due to their production of inflammatory mediators such as IL-6 and prostaglandins. The resulting inflammation recruits immunosuppressive cells that also release cytokines and feed the inflammatory environment. As solid tumors outpace their blood supply and become deprived of nutrients and oxygen, necrosis sets in causing the chronic release of pro-inflammatory mediators such as IL-1 and high-mobility group box 1 (HMGB1), which in turn promote neoangiogenesis (Vakkila \& Lotze, 2004). Long-term infection may also cause chronic inflammation and increased cancer risk. Examples include hepatocellular carcinoma in patients infected with hepatitis B or C viruses 
(Karin, 2006), and bladder and colon cancer in individuals infected with Schistosoma or Bacteroides, respectively (Mostafa, Sheweita, \& O'Connor, 1999; Wu et al., 2009).

Chronic inflammation promotes tumor development through various mechanisms including the production of proangiogenic factors, matrix metalloproteinases (MMPs), and damage-associated molecular pattern molecules (DAMPs), all of which drive MDSC accumulation and MDSC suppressive functions. Proangiogenic factors such as VEGF stimulate tumor neovascularization, while MMPs facilitate invasion and metastasis (Shacter \& Weitzman, 2002). DAMPs such as S100A8/A9 chemoattract leukocytes and promote the expansion of MDSCs leading to an influx of inflammatory molecules within the TME (Cheng et al., 2008; Sinha et al., 2008).

The TME is a complex network that includes both tumor cells and host cells. MDSCs in this environment are therefore subjected to diverse proinflammatory factors. Since the TME varies between tumor types and individuals with cancer, as well as with stage of tumor progression, it is not surprising that MDSCs are a heterogeneous population that may vary from individual to individual.

Almost a decade ago, the connection between MDSCs and inflammation was established with the findings that the pro-inflammatory cytokines IL- $1 \beta$, $\mathrm{IL}-6$, and $\mathrm{PGE}_{2}$ promote MDSC accumulation and suppressive function (Bunt, Sinha, Clements, Leips, \& Ostrand-Rosenberg, 2006; Bunt et al., 2007; Ezernitchi et al., 2006; Sinha, Clements, Fulton, \& OstrandRosenberg, 2007; Song et al., 2005). Other studies demonstrated that additional cytokines, transcription factors, and DAMPs, including, but not limited to, C5a, PGE 2 , COX 2 , VEGF, GM-CSF, G-CSF, IL-17, IDO, HMGB1, and S100A8/A9, C/EBP $\beta$, and chop, also drive MDSCs.

The effects of these factors are discussed in the following section and are illustrated in Fig. 2.

\subsection{Vascular Endothelial Growth Factor}

VEGF is a pro-inflammatory growth factor that stimulates angiogenesis, and tumors producing high levels of VEGF have a poor prognosis. VEGF inhibits nuclear factor kappa-light-chain-enhancer (NF- $\mathrm{kB}$ ) activation which blocks DC development while simultaneously driving MDSC accumulation (Gabrilovich et al., 1998). MDSCs express the VEGF receptor enabling VEGF to function as a chemoattractant for MDSCs. ROS 


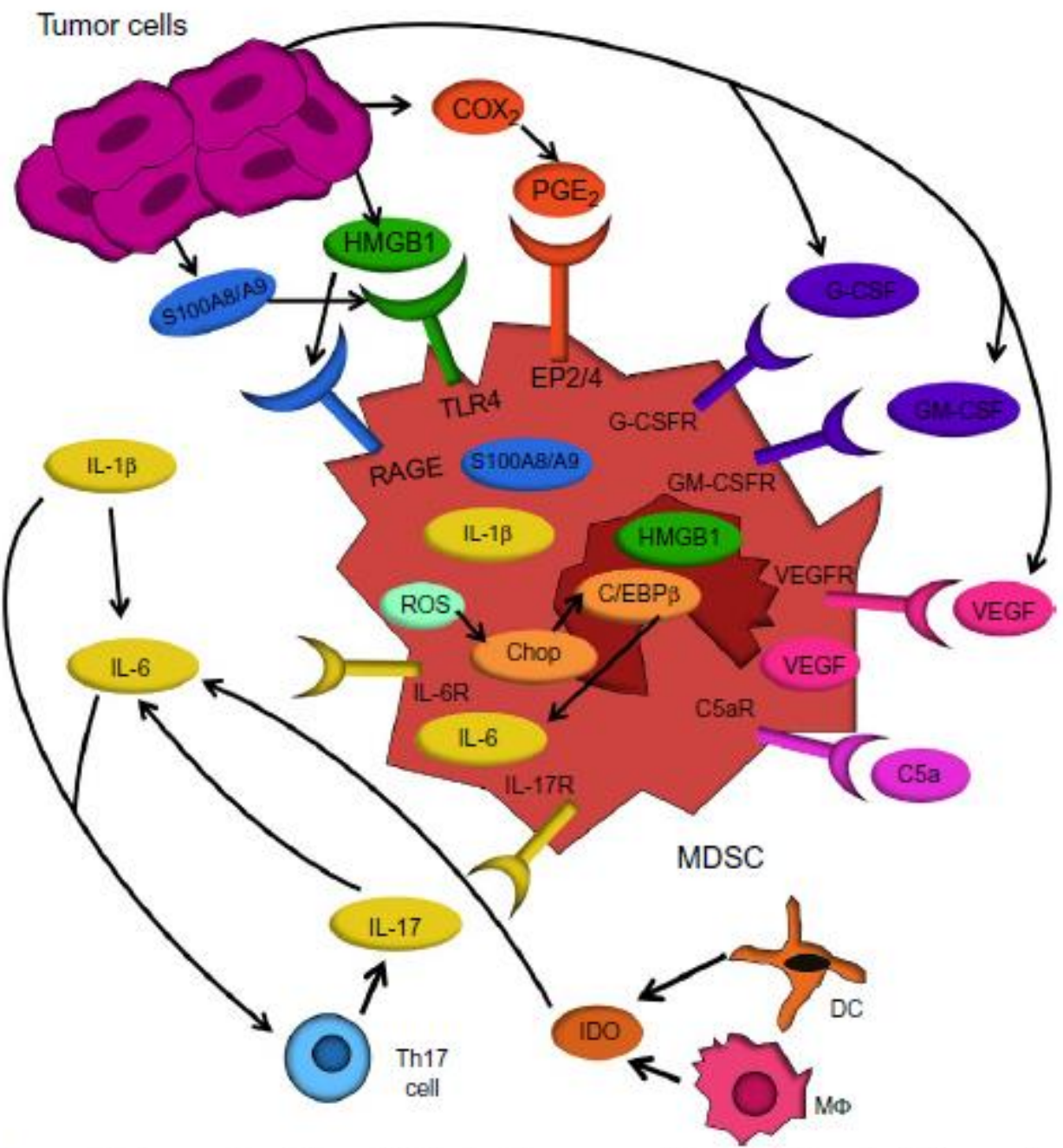

Figure 2 Inflammation drives MDSC development and function. Chronic inflammation induces the production of HMGB1, S100A8/A9, IL-1 $\beta, \mathrm{IL}-6, \mathrm{CS}$, and IL-17, all of which induce the accumulation of MDSC. Induction of MDSCS by IL-1 $\beta$ is mediated through IL-17 and IL-6. IL- $1 \beta$ induces Th17 cells to produce IL-17 which induces the production of IL-6. IL-6 production is also upregulated by IDO produced by DCs and macrophages (MФ). C/EBP $\beta$, which is activated by chop following MDSC production of ROS, also induces IL-6. MDSCs also produce VEGF, IL-6, IL-1 $\beta, H M G B 1$, and S100A8/A9, thereby perpetuating their accumulation. Tumor cells may produce $\mathrm{COX}_{2}, \mathrm{PGE}_{2}, \mathrm{VEGF}$, IL-6, G-CSF, GM-CSF, S100A8/A9, and HMGB1 all of which induce the accumulation of MDSCs and may increase the suppressive potency of MDSCs. 
production by MDSCs increases oxidative stress which upregulates MDSC expression of the VEGF receptor (Kusmartsev et al., 2008). Since other factors in solid tumors also contribute to oxidative stress, the TME is a critical factor in determining the responsiveness of MDSCs to VEGF.

In addition to tumor cells, MDSCs themselves produce VEGF, thereby creating an autocrine feedback loop that sustains MDSC accumulation (Kujawski et al., 2008). VEGF has been shown to be released from the extracellular matrix by MMP9, a matrix degrading enzyme (Bergers et al., 2000). Soluble MMP9 is produced by tumor cells and promotes MDSC accumulation and tumor angiogenesis (Melani, Sangaletti, Barazzetta, Werb, \& Colombo, 2007). Therefore, MDSCs have multiple modes of generating VEGF.

\subsection{Granulocyte-Macrophage Colony-Stimulating Factor and Granulocyte Colony-Stimulating Factor}

GM-CSF is a growth factor for leukocytes. It is required for DC differentiation and is used to expand DC ex vivo. However, high levels of GM-CSF induce MDSC accumulation in vivo and in vitro, while in vivo knockdown of GM-CSF reduces MDSC expansion (Morales, Kmieciak, Knutson, Bear, \& Manjili, 2010; Serafini et al., 2004). Inclusion of GM-CSF in cultures of bone marrow progenitor cells drives the differentiation of MDSCs, demonstrating that GM-CSF is a growth factor for MDSCs (Nefedova et al., 2004).

MDSC differentiation is also positively regulated by the growth factor granulocyte colony-stimulating factor (G-CSF). G-CSF plays a critical role in mobilizing bone marrow stem cells and is essential for differentiation of granulocytic lineages (Lieschke et al., 1994). Administration of G-CSF to tumor-bearing mice drives tumor growth and angiogenesis, while blockade of G-CSF reduces MDSC levels (Okazaki et al., 2006). G-CSF also preconditions metastatic sites by mobilizing MDSCs (Kowanetz et al., 2010). When G-CSF and VEGF are both inhibited, tumor growth is reduced (Okazaki et al., 2006). While the role of G-CSF in MDSC development is clear, the impact of G-CSF on MDSC function is more complicated. In mice bearing MCA203 sarcomas, G-CSF induced $\mathrm{Gr} 1^{\text {hi }} \mathrm{CD} 11 \mathrm{~b}^{+}$ cells that were less suppressive than $\mathrm{Gr} 1^{\text {int }} \mathrm{CD} 11 \mathrm{~b}^{+}$cells, while in MMTV-PyMT transgenic mice with mammary carcinoma, G-CSF caused CD11 ${ }^{+}{\text {Ly } 6 G^{+}}^{+}$Ly6C ${ }^{+}$cells to secret Bv8. Bv8 is an endocrine analog of VEGF and functions as a proangiogenic protein that promotes hematopoiesis (Dolcetti et al., 2010; Kowanetz et al., 2010). Therefore, G-CSF differentially affects MDSC function depending on the type of tumor. 


\subsection{Prostaglandin E2 and Cyclooxygenase 2}

Prostaglandin E2 $\left(\mathrm{PGE}_{2}\right)$ is a potent inflammatory mediator that is generated by cyclooxygenase $2\left(\mathrm{COX}_{2}\right)$ conversion of arachidonic acid. $\mathrm{PGE}_{2}$ supports tumor growth by promoting angiogenesis, stimulating tumor-cell proliferation, and protecting tumor cells from apoptosis. Many human and mouse tumors as well as tumor-infiltrating cells produce $\mathrm{COX}_{2}$ and $\mathrm{PGE}_{2}$. $\mathrm{PGE}_{2}$ promotes MDSC differentiation at the expense of DC, while inhibition of $\mathrm{COX}_{2}$ or $\mathrm{PGE}_{2}$ in tumor-bearing mice blocks MDSC differentiation and delays tumor progression (Eruslanov, Daurkin, Ortiz, Vieweg, \& Kusmartsev, 2010; Sinha, Clements, Fulton, et al., 2007). In the TME, $\mathrm{PGE}_{2}$ mediates its effects through four integral membrane G-protein-coupled prostanoid receptors: EP1, EP2, EP3, and EP4. Mice deficient in EP2 display delayed tumor progression and reduced MDSC levels (Sinha, Clements, Fulton, et al., 2007). Blockade of $\mathrm{PGE}_{2}$ or EP4 in tumor-bearing mice reduces MDSC production of ARG1 (Rodriguez et al., 2005). $\mathrm{PGE}_{2}$ promotes the differentiation of progenitor cells in human blood to MDSCs (CD11 ${ }^{+} \mathrm{CD} 33^{+}$cells) from human blood progenitor cells that have elevated levels of NOS2, ARG1, IL-10, and IL-4R $\alpha$ (Obermajer, Muthuswamy, Lesnock, Edwards, \& Kalinski, 2011). Therefore, for mouse and human MDSCs, $\mathrm{PGE}_{2}$ not only regulates the differentiation of MDSCs, but several suppressive mechanisms as well.

\subsection{CCAAT/Enhancer Binding Protein $\beta$ and C/EBP Homologous Protein}

$\mathrm{C} / \mathrm{EBP}$ proteins are a family of leucine zipper transcription factors that regulate inflammation and myeloid cell differentiation. While there are various isoforms of $\mathrm{C} / \mathrm{EBP}$ proteins, CCAAT/enhancer binding protein $\beta$ $(\mathrm{C} / \mathrm{EBP} \beta)$ acts during stress/inflammation-induced myelopoiesis. $\mathrm{C} / \mathrm{EBP} \beta$ has three isoforms: LAP* and LAP (liver-enriched activator proteins), and LIP (liver-enriched inhibitory protein). LAP* and LIP are transcriptional activators that drive inflammatory myelopoiesis by inducing IL-6 and ARG1. In contrast, LIP inhibits LAP signaling promoting an antiinflammatory response. In inflammatory settings such as the TME, LAP* and LAP are active and drive inflammation-induced myelopoiesis. C/EBP $\beta$ is also required for the ex vivo generation of immunosuppressive MDSCs from bone marrow progenitor cells, via IL-6 and GM-CSF (Marigo et al. 2010). 


\subsection{Complement Component C5a}

C5a (also known as anaphylatoxin) is a pro-inflammatory member of the complement and lectin pathway. When the complement pathway is activated, C5a in the blood becomes fixed in tissues. C5a triggers degranulation of mast cells (MCs), aids in vascular permeability, and stimulates smooth muscle contraction. In a tumor setting, C5a increases MDSC-mediated

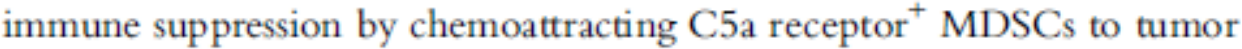
vasculature and by increasing MDSC production of ROS and ARG1 (Markiewski et al., 2008).

\subsection{S100A8/A9}

S100A8/A9 proteins are pro-inflammatory danger signals. They are calcium binding proteins that are localized in the cytoplasm or nucleus of myeloid cells, and are released in response to cell damage, infection, or inflammation. Mice deficient in S100A9 reject transplanted tumors, while elevated expression of S100A8/A9 in solid tumors perpetuates inflammation by chemoattracting leukocytes that produce additional inflammatory molecules (Cheng et al., 2008; Sinha et al., 2008). MDSCs are one of the leukocyte populations that are chemoattracted by $\mathrm{S} 100 \mathrm{~A} 8 / \mathrm{A} 9$, and chemoattraction is dependent on signaling through receptor for advanced glycation endproducts (RAGE) (Sinha et al., 2008). Heterodimeric S100A8/A9 mediates it's pro-inflammatory effects by binding to the plasma membrane receptors TLR4, carboxylated N-glycans, RAGE, or heparin sulfate (Bresnick, Weber, \& Zimmer, 2015). MDSCs amplify their own accumulation by secreting S100A8/A9, thus creating a self-sustained feedback loop (Sinha et al., 2008).

\subsection{High-Mobility Group Box 1}

HMGB1 is the second most abundant protein within a cell and is released from myeloid cells as a danger response to sepsis, infection, or arthritis. HMGB1 can signal through a number of receptors including thrombospondin, CD24, TLR2, 4, 7, and 9, as well as RAGE. HMGB1 consists of two functional domains, the $\mathrm{A}$ and $\mathrm{B}$ boxes, and an acidic tail. The $\mathrm{A}$ box is a RAGE antagonist and prevents HMGB1-mediated release of IL-1 $\beta$ and TNF $\alpha$. The B box and part of the linker before the acidic tail is a RAGE agonist with pro-inflammatory properties (Bianchi \& Manfredi, 2007). The B box signals via TLR 4 on macrophages which initiate the release of 
$\mathrm{IL}-1 \beta, \mathrm{IL}-6$, TNF $\alpha, \mathrm{MIP}-2$, and IL-10. The A box is anti-inflammatory as it prevents HMGB1-mediated release of IL $-1 \beta$ and TNF $\alpha$. Whether HMGB1 functions in a pro-inflammatory or anti-inflammatory manner is determined by its redox state. In the normal extracellular environment, the disulfide bridge between residues $\mathrm{Cys}_{23}$ and $\mathrm{Cys}_{45}$ maintains the $\mathrm{A}$ box in a dysfunctional conformation, so the B box is exclusively active. With inflammation, the microenvironment becomes oxidatively stressed and ROS is produced. ROS terminally oxidizes $\mathrm{Cys}_{23}$ and $\mathrm{Cys}_{45}$, thereby breaking the disulfide bridge and allowing $\mathrm{A}$ box to resolve the inflammation (Venereau et al., 2012).

Elevated levels of HMGB1 are associated with numerous cancers and are known to directly promote tumor growth. However, HMGB1 also drives tumor progression by modulating MDSCs. Inhibition of HMGB1 prevents the expansion of MDSCs from bone marrow progenitor cells in vitro, demonstrating that HMGB1 is required for the differentiation of MDSCs. In vivo inhibition of HMGB1 in tumor-bearing mice reduces MDSC levels in the tumor, spleen, and blood, confirming HMGB1 as a driver of MDSCs. MDSC-mediated downregulation of $\mathrm{T}$ cell L-selectin (CD62L) is also HMGB1 dependent, since HMGB1 increases MDSC extracellular expression of A disintegrin and metalloproteinase 17 (ADAM17), a protease that cleaves L-selectin. Secretion of the protumor cytokines IL-10 and IL- $1 \beta$ by MDSCs is also increased by HMGB1 (Parker, Sinha, Horn, Clements, \& Ostrand-Rosenberg, 2014), and HMGB1-driven MDSC accumulation facilitates metastasis (Li et al., 2013). Preliminary studies indicate that HMGB1 mediates its effects on MDSCs through RAGE and/or TLR4 (K.H. Parker \& S. Ostrand-Rosenberg, unpublished). HMGB1 also binds to other receptors, but it is unknown if MDSCs are activated through additional receptors.

\subsection{IL-1 $\beta$, IL-6, and Indoleamine 2,3-Dioxygenase}

The causative relationship between inflammation, cancer, and immune suppression was first proposed following the finding that IL- $1 \beta$ was a potent inducer of MDSC accumulation and suppressive activity (OstrandRosenberg \& Sinha, 2009). Mice bearing 4T1 tumor cells that were transfected to constitutively express high levels of IL- $1 \beta$ exhibit increased MDSC accumulation and more suppressive MDSCs compared to mice bearing parental 4T1 tumors. 4T1 tumor-bearing mice that lack the $\mathrm{IL}-1$ receptor 
antagonist, an inhibitor for IL-1 $\beta$, also develop elevated levels of MDSCs that are more suppressive. Similarly, mice deficient for the IL-1R display slower tumor growth and their MDSCs are less suppressive (Bunt et al., 2006, 2007; Elkabets et al., 2010; Song et al., 2005). Since IL-1 $\beta$ induces the production of other mediators, including VEGF, IL-6, $\mathrm{PGE}_{2}$, and GM-CSF, some of the effects of IL- $1 \beta$ on MDSCs may be indirect. 4T1 tumor cells transfected to constitutively express IL-6 induce elevated levels of MDSCs and restore MDSC levels in tumor-bearing $\mathrm{IL}-1$ receptor knockout mice, indicating that IL-6 effects on MDSCs are either downstream of $\mathrm{IL}-1 \beta$, or have an overlapping mechanism of action with $\mathrm{IL}-1 \beta$ (Bunt et al., $2007)$. Since MDSCs produce IL -6 and IL $-1 \beta$, these studies also raise the question of whether MDSC production of $\mathrm{IL}-6$ is regulated by $\mathrm{IL}-1 \beta$, and if MDSC production of IL-1 $\beta$ enhances MDSC production of IL-6. Indole amine 2,3 dioxygenase (IDO), which is utilized by MDSCs as an immune-suppressive mechanism, also regulates IL-6, and tumor-bearing IDO1-deficient mice have less suppressive MDSCs, reduced levels of $\mathrm{IL}-6$, and delayed primary tumor growth and metastatic disease (Smith et al., 2012). Provision of IL-6 to tumor-bearing indoleamine 2,3dioxygenase (IDO) knockout mice restores MDSC levels and suppressive potency (Smith et al., 2012).

\subsection{IL-17}

IL-17 is a pro-inflammatory cytokine secreted by CD4 Th17 and CD8 Tc17 cells. Tumor growth is suppressed and MDSC levels are decreased in IL-17-deficient mice, while administration of IL-17 raises MDSC levels (He et al., 2010; Wang et al., 2009). Patients with gastrointestinal cancers show a strong positive correlation between serum IL-17 and MDSC levels, further supporting a role for IL-17 as an inducer of MDSCs (Yazawa et al., 2013). The effects of IL-17 may be either direct or indirect. Most cells have IL-17 receptors so MDSCs may be directly impacted. However, IL-17 triggers the production of IL-6 which in turn activates STAT3, so many effects on MDSCs may be directly mediated by IL -6 and indirectly by IL -17 (Chatterjee et al., 2013; Wang et al., 2009).

\section{MDSC ARE REGULATED BY MULTIPLE MOLECULAR}

Multiple signal transduction pathways, transcription factors, and microRNAs (miRNAs) regulate MDSC accumulation and function (Fig. 3). 


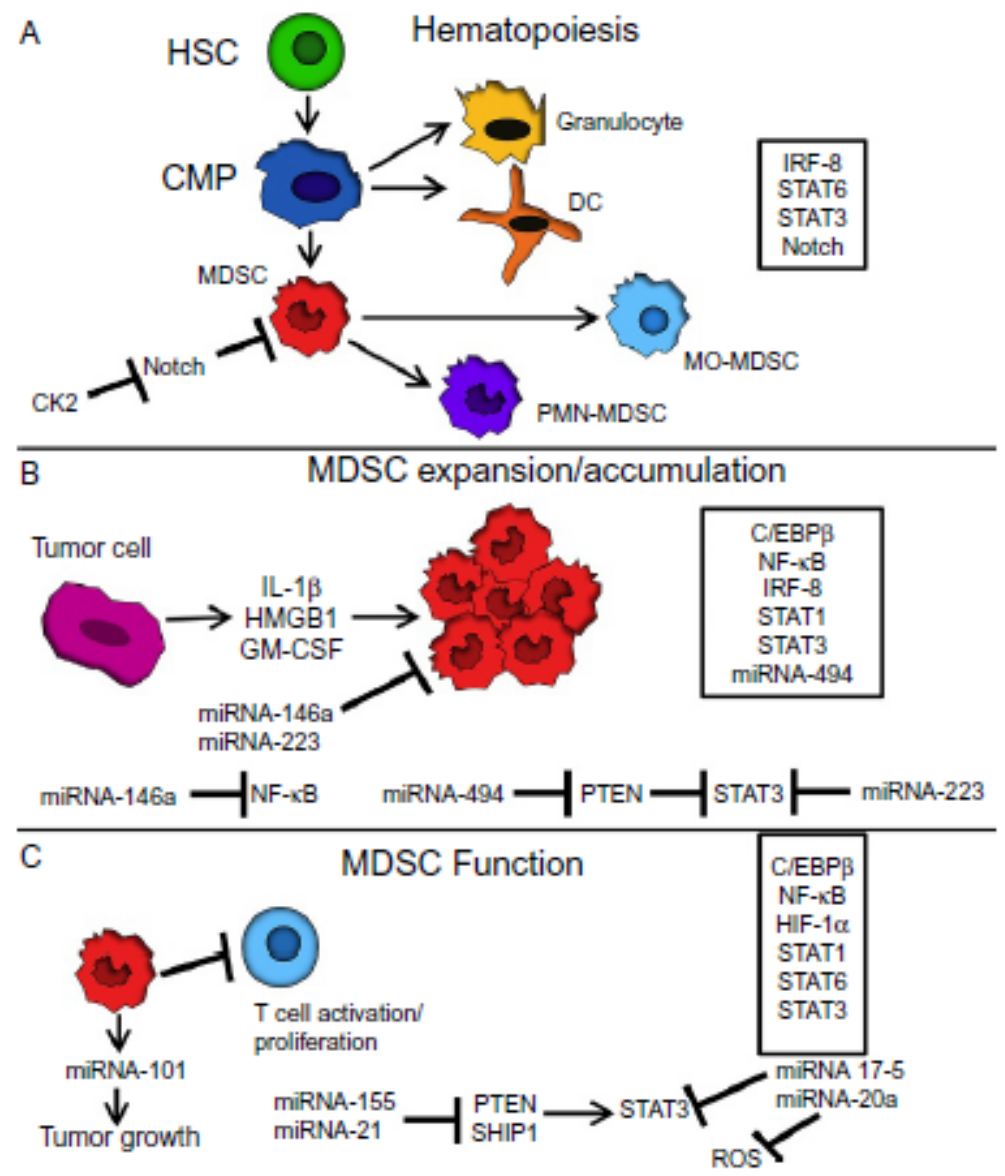

Figure 3 Multiple signal transduction pathways, transcription factors, and microRNAs regulate MDSC accumulation and function. (A) The differentiation of MDSCs from HSC and CMP is regulated by the transcription factors IRF-8, Notch, STAT6, and STAT3. IRF-8 and STAT6 regulate normal myelopoiesis and the differentiation of CMP to mature granulocytes and DCs. During abnormal myelopoiesis, which occurs in individuals with cancer, immature myeloid cells fail to terminally differentiate giving rise to elevated levels of MDSCs. Notch inhibits the differentiation of MDSCs, while CK2 blocks Notch and thereby increases MDSCs. STAT3 promotes MDSC development and suppressive potency. (B) Tumor and host cells produce multiple inflammatory molecules that perturb myelopoiesis and induce the expansion of MDSCs by activating or inactivating transcription factors. Pro-inflammatory mediators in the tumor microenvironment, such as $\mathrm{IL}-1 \beta, \mathrm{HMGB} 1$, and GM-CSF, drive the expansion of MDSCs by activating C/EBP $\beta, N F-\mathrm{kB}$, STAT1, STAT3, and miRNA-494 and downregulating IRF-8. Induction of miRNAs 146a and 223 prevents the expansion of MDSC. miRNA-494 promotes the expression of MMPs and inhibits PTEN function resulting in STAT3 induction. miRNA-146a inhibits NF- $\mathrm{kB}$ signaling, while miRNA-223 blocks C/EBP $\beta$ from binding to the c-myc promoter which downregulates STAT3 expression. (C) MDSC function is positively regulated by C/EBP $\beta$, NF-kB, HIF- $1 \alpha$, STAT1, STAT6, and STAT3. The miRNAs 155 and 21 inhibit PTEN and SHIP1, negative regulators of STAT3, resulting in the activation of STAT3 and increased MDSC function. miRNAs 17-5 and 20a have the opposite effect by blocking STAT3 and ROS which negatively regulates MDSC function. MDSCs themselves also promote tumor growth by activating miRNA-101 in cancer cells. 


\subsection{Signal Transducer and Activator of Transcription 1}

MDSC function is positively regulated by STAT1. STAT1 is activated by IFN $\gamma$ or $\mathrm{IL}-1 \beta$ and regulates the induction of NOS2 and ARG1 (Kusmartsev \& Gabrilovich, 2005). MDSC accumulation is also dependent on STAT1 as tumor-bearing mice deficient in STAT1 exhibit reduced MDSC levels (Hix et al., 2013). Whether IFN $\gamma$ is the ligand that activates MO-MDSCs is unclear. Early studies indicated that IFN $\gamma$ was essential for the development of MO-MDSCs (Movahedi et al., 2008); however, subsequent experiments demonstrated that MDSC function, accumulation, and phenotype are independent of IFN $\gamma$ as tumor-bearing $\operatorname{IFN} \gamma^{+/+}, \operatorname{IFN} \gamma^{-/-}$, IFN $\gamma \mathrm{R}^{+/+}$, and IFN $\gamma \mathrm{R}^{-/-}$mice with equal-sized tumors contained equal numbers of equivalently suppressive MDSCs (Sinha, Parker, Horn, \& Ostrand-Rosenberg, 2012).

\subsection{Signal Transducer and Activator of Transcription 3 and 6}

MDSC accumulation and function are enhanced by activation of both STAT3 and STAT6. Activation of STAT6 occurs from the binding of $\mathrm{IL}-4$ or $\mathrm{IL}-13$ to $\mathrm{IL}-4 \mathrm{R} \alpha$ resulting in the upregulation of ARG1 and TGF $\beta$ (Bronte et al., 2003; Sinha, Clements, \& Ostrand-Rosenberg, 2005a; Terabe et al., 2003). In STAT6-deficient mice, signaling through IL-4R $\alpha$ does not occur, MDSCs are less suppressive and accumulate more slowly, and spontaneous metastatic disease is delayed (Sinha, Clements, \& Ostrand-Rosenberg, 2005b). STAT3 activation increases the half-life and proliferation of both human and mouse MDSCs by driving the expression of the antiapoptotic genes $\mathrm{Bcl}-\mathrm{xL}, \mathrm{c}-\mathrm{myc}$, and the proliferation gene cyclin D1 (Nefedova et al., 2005; Xin et al., 2009). STAT3 also increases the differentiation of MDSCs by inducing the pro-inflammatory mediators S100A8/A9 (Cheng et al., 2008) and by downregulating the transcription factor PKC $\beta$ II in hematopoietic progenitor cells (Farren, Carlson, \& Lee, 2010). Since MDSCs and DCs are derived from a common progenitor cell, the increase in MDSC differentiation is accompanied by a decrease in DC expansion. In addition to regulating MDSC expansion, STAT3 also enhances MDSC suppressive activity (Kujawski et al., 2008). Tumorderived exosomes containing heat-shock protein 72 on their membranes induce MDSC production of IL-6 which subsequently activates STAT3 and increases MDSC-mediated T cell suppression (Chalmin et al., 2010).

$\mathrm{C} / \mathrm{EBP} \beta$ is another transcription factor activated by STAT3. Activated $\mathrm{C} / \mathrm{EBP} \beta$ binds to the $\mathrm{c}-\mathrm{myc}$ promoter and induces $\mathrm{c}-\mathrm{myc}$ expression which 
stimulates cell proliferation. $\mathrm{C} / \mathrm{EBP} \beta$ is a key molecule for induction of MDSCs since multiple factors (GM-CSF, G-CSF, and IL-6) activate MDSCs via $\mathrm{C} / \mathrm{EBP} \beta$, (Marigo et al., 2010). C/EBP $\beta$ regulation of MDSCs is associated with chop. ROS produced by tumors upregulates MDSC expression of chop (Thevenot et al., 2014). Chop expression in MDSCs activates $\mathrm{C} / \mathrm{EBP} \beta$ and induces STAT3 signaling. MDSCs from chopdeficient mice have decreased ability to inhibit T cell proliferation and accumulate to lower levels. This reduced accumulation and decreased potency of MDSCs is attributed to lower levels of IL-6 and reduced phosphorylation of STAT3. Overexpression of IL-6 in chop-deficient mice rescues MDSC suppressive activity (Thevenot et al., 2014).

MDSC production of ROS is also regulated by STAT3. ROS are generated intracellularly by the $\mathrm{NAD}(\mathrm{P}) \mathrm{H}$ oxidase enzyme complex (NOX), which consists of membrane-bound gp91 and p22, and cytosolic p40, $\mathrm{p} 47$, and $\mathrm{p} 67$. This complex catalyzes the production of superoxide through the reduction of oxygen, with $\mathrm{NAD}(\mathrm{P}) \mathrm{H}$ serving as the one electron donor. Activation of STAT3 increases ROS levels through upregulation of p47 and gp91 (Corzo et al., 2009); however, it is not known which of the several activators of STAT3 upregulate $\mathrm{p} 47$ and gp91. Solid tumors contain oxidatively stressed hypoxic regions, and cells within these regions contain activated hypoxia-inducible factor- 1 alpha (HIF-1 $\alpha$ ). Activated HIF-1 $\alpha$ induces STAT3 signaling. Therefore, STAT3 induction of ROS may be regulated by $\mathrm{HIF}-1 \alpha$.

\subsection{Nuclear Factor Kappa-Light-Chain-Enhancer}

Activation of NF- $\mathrm{KB}$ also promotes MDSC accumulation and function and occurs following ligation of MyD88-dependent TLRs. Exposure to a variety of pro-inflammatory mediators including S100A8/A9, HMGB1, and IL-1 $\beta$ activates the NF- $\kappa$ B pathway in MDSCs (Parker et al., 2014; Sinha et al., 2008; Tu et al., 2008).

\subsection{Interferon Regulatory Factor-8}

Interferon regulatory factor 8 (IRF-8) is a transcription factor that is essential for the normal development of granulocyte/monocyte lineage cells. IRF-8deficient mice have myeloproliferative disorders and accumulate high levels of MDSCs. Expression of IRF-8 is downregulated by G-CSF and GM-CSF, so treating mice with these cytokines blocks IRF-8 activation and drives the accumulation of MDSCs (Stewart, Liewehr, Steinberg, Greeneltch, \& 
Abrams, 2009; Waight et al., 2013). IRF-8 may also negatively regulate MDSC survival as IRF-8 downregulates antiapoptotic genes $\mathrm{Bcl}-2$ and $\mathrm{Bcl}-\mathrm{xL}$ and upregulates the proapoptotic gene caspase-3 (Burchert et al., 2004; Gabriele et al., 1999). Inhibition of Bcl-2 and $\mathrm{Bcl}-\mathrm{xL}$ enhances MDSC susceptibility to Fas-mediated apoptosis (Hu et al., 2013).

\subsection{Notch}

Another transcription factor implicated in the development of MDSCs from hematopoietic progenitor cells is Notch. Notch signaling permits the differentiation of MDSCs into DCs. Inhibition of notch signaling by casein kinase 2 (CK2) drives abnormal myeloid cell differentiation (Cheng et al., 2014).

\subsection{Hypoxia-Inducible Factor-1 Alpha}

The HIF complex consists of the subunits HIF- $1 \alpha$ and HIF- $1 \beta$, both of which are constitutively expressed. Hypoxia stabilizes HIF- $1 \alpha$ and allows it to translocate from the cytoplasm into the nucleus where it dimerizes with HIF-1 $\beta$. The HIF complex upregulates multiple target genes (e.g., VEGF, NOS2, and MMPs) by associating with their hypoxia response elements. HIF- $1 \alpha$ is overexpressed in various cancers, where it increases MDSC expression of ARG1 and NOS2, rendering MDSCs more immune suppressive and facilitating their conversion to tumor-associated macrophages (TAMs) (Corzo et al., 2010). The capacity of HIF-1 $\alpha$ to modulate the function of MDSCs highlights the plasticity of MDSCs and further demonstrates that MDSC function is governed by their environment.

\subsection{MicroRNAs}

miRNAs are noncoding single-stranded RNAs approximately 22 nucleotides long that regulate gene expression. miRNAs in the RNA-induced silencing complex bind to complementary target mRNAs causing target mRNA degradation. The generation of miRNAs is regulated by celland tissue-specific transcription factors as well as proteins involved in the processing of miRNA, both of which can be influenced by chronic inflammation (El Gazzar \& McCall, 2012).

miRNAs enhance and inhibit MDSC accumulation and suppressive potency. For example, miRNAs $146 a$ and 223 prevent MDSC accumulation (Boldin et al., 2011; Liu et al., 2011). miRNA-146a blocks inflammation, while miRNA-223 is needed for the development of granulocytes. In contrast, miRNAs 494, 155, and 21 facilitate the accumulation of MDSCs. 
miRNA-494 induces MMPs 2, 13, and 14 which drive MDSC growth and survival signals, and by inhibiting phosphatase and tensin homolog (PTEN) which promotes STAT3 activation (Liu et al., 2012). miRNAs 155 and 21 promote MDSC accumulation by activating STAT3, which, as previously discussed, drives both MDSC accumulation and suppressive potency (Li et al., 2014). miRNAs also negatively regulate MDSC suppressive function. These include miRNAs $17-5$ and $20 \mathrm{a}$ which silence STAT3, thereby reducing MDSC production of ROS and hydrogen peroxide $\left(\mathrm{H}_{2} \mathrm{O}_{2}\right)$ (Zhang et al., 2011).

MDSCs also use miRNAs to modulate cancer cell growth. An example is the MDSC-mediated activation of miRNA-101 in ovarian cancer cells (Cui et al., 2013). miRNA-101 increases cancer cell stemness as well as metastatic and tumorigenic potential (Cui et al., 2013).

\subsection{MDSC Turnover}

MDSC turnover in vitro and in vivo varies with tumor type, with half-life ranging for only a few days. T cells may contribute to this rapid turnover since when activated, T cells express FasL and cause apoptosis of Fas ${ }^{+}$ MDSCs. Inflammation counteracts the $\mathrm{T}$ cell effect by increasing MDSC resistance to Fas-mediated lysis (Chomoguz et al., 2011; Sinha et al., 2011).

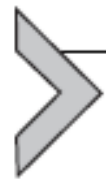

\section{MDSCS UTILIZE A NETWORK OF EFFECTOR AND SIGNALING MOLECULES TO MODULATE THE INFLAMMATORY MILIEU AND DECREASE IMMUNE SURVEILLANCE}

MDSCs utilize multiple suppressive mechanisms to induce a tolerogenic, tumor-promoting environment. MDSCs directly suppress T cells by starving them of amino acids, inducing apoptosis, reducing homing to lymph nodes, or inhibiting their intracellular signaling pathways required for activation. MDSCs also indirectly suppress $\mathrm{T}$ cells by altering the ability of antigen-presenting cells (APCs) to activate T cells and by inducing immunosuppressive $\mathrm{T}$ regulatory cells (Tregs). In addition, MDSCs impact other cells involved in an antitumor response because they alter the inflammatory milieu in the TME by cross talk with macrophages, tumor cells, and MCs. These mechanisms are described below and are illustrated in Fig. 4. 


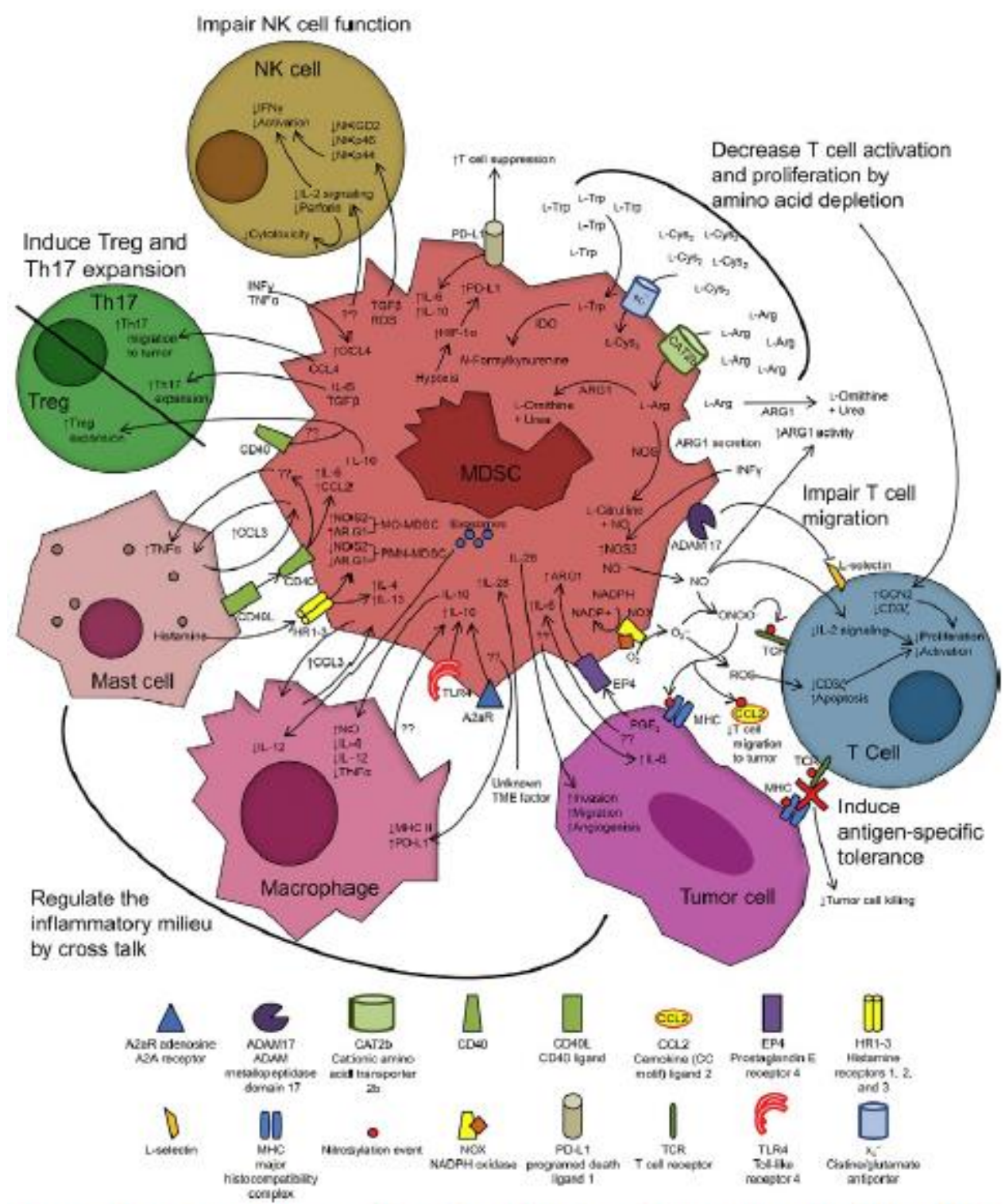

Figure 4 MDSCs suppress $T$ cells and regulate the inflammatory milieu by multiple mechanisms. MDSCs regulate antitumor immunity by (i) secretion of IL-10, which induces Tregs; (ii) secretion of IL-6 and TGF $\beta$, which induces Th17 cells; (iii) production of ROS and TGF $\beta$, which inhibits NK cell function; (iv) degradation of amino acids essential for $\mathrm{T}$ cell activation and proliferation; (v) production of $\mathrm{NO}$ and $\mathrm{O}_{2}{ }^{-}$, which induces apoptosis and inhibits the activation and proliferation of $\mathrm{T}$ cells, and generates PNT that nitrates/nitrosylates MHC and TCR; and (vi) participation in cross talk with macrophages, tumor cells, and mast cells to generate a protumor environment. Question marks denote an unknown mechanism or signaling molecule. 


\subsection{MDSC Depletion of Amino Acids}

Following initial contact with antigen, $\mathrm{T}$ cells undergo metabolic changes that are essential for their activation and clonal expansion. MDSCs limit several amino acids necessary for these processes and thereby inhibit $\mathrm{T}$ cell activation. An early event of amino-acid starvation is the accumulation of empty aminoacyl tRNAs, which activate serine-threonine kinase GCN2. GCN2 phosphorylates eIF2 $\alpha$, which binds eIF2B and suppresses the translation initiation complex from binding charged aminoacyl tRNA, thereby causing a global decrease in protein translation. Simultaneously, GCN2 enhances the translation of GCN4, which results in the transcription of genes required for the synthesis of amino acids (Wang \& Green, 2012). MDSCs deplete the local environment of L-arginine (L-Arg), L-tryptophan (L-Trp), and L-cysteine (L-Cys) through different mechanisms.

One of the first suppressive mechanisms attributed to MDSCs was the inhibition of $\mathrm{T}$ cell activation and proliferation by the depletion of $\mathrm{L}-\mathrm{Arg}$. L-Arg is a nonessential amino acid and is a substrate for several enzymes: (i) NOS 1, 2, and 3 which metabolize L-Arg into L-citrulline and nitric oxide (NO); (ii) ARG 1 and 2 which convert L-Arg to L-ornithine and urea; (iii) arginine:glycine amidinotransferase which transfers the amidino group from L-Arg to L-glycine, yielding L-ornithine and glycocyamine; and (iv) arginine decarboxylase, which catalyzes the reaction of L-Arg to agmatine and $\mathrm{CO}_{2}$ (Bronte \& Zanovello, 2005).

In the absence of L-Arg, T cells decrease their expression of CD3 3 , which is required for signal transduction through the antigen-specific T cell receptor (TCR) (Rodriguez et al., 2002; Zea et al., 2004). L-Arg-depleted T cells are arrested in $\mathrm{G}_{0}-\mathrm{G}_{1}$ due to the failure to upregulate cyclin D3 and cyclindependent kinase 4 (cdk4). Cyclin D3 and cdk4 are not upregulated due to decreased mRNA stability and lower translation rates (Rodriguez et al., 2010). Despite their inability to proliferate, L-Arg-starved T cells express early activation markers and secrete IL-2, indicating that the early events of $\mathrm{T}$ cell activation are not L-Arg dependent (Fletcher et al., 2015). In vivo studies confirmed the critical role of MDSCs in L-Arg depletion since renal cell carcinoma patients and mice with chronic inflammation have elevated levels of MDSCs and low levels of serum L-Arg, which is correlated with decreased T cell activation (Ezernitchi et al., 2006; Zea et al., 2005). Depletion of L-Arg is mediated by ARG1, and MDSC synthesis of ARG1 is regulated by $\mathrm{PGE}_{2}$ (Rodriguez et al, 2005). Tumor-derived MDSCs deplete their local environment of L-Arg by internalizing L-Arg 
through the cationic amino-acid transporter 2B (Rodriguez et al., 2004) and by secreting ARG1 (Rodriguez et al., 2009).

L-Trp metabolism by MDSCs also facilitates T cell suppression. MDSCs express IDO, which degrades the essential amino-acid L-Trp into $\mathrm{N}$-formylkynurenine. IDO causes $\mathrm{T}$ cell suppression by enhancing GCN2 kinase in a similar manner as L-Arg starvation (Munn et al., 2005). Expression of IDO in MDSCs is regulated by STAT3 (Yu et al., 2013). However, not all MDSCs express IDO (Smith et al., 2012), indicating that IDO is not a universal mechanism utilized by MDSCs to suppress T cell activation.

MDSCs also prevent $\mathrm{T}$ cell activation by sequestering $\mathrm{L}-\mathrm{Cys}$. In the extracellular oxidizing environment, L-Cys exists as the dipeptide cystine $\left(\mathrm{L}-\mathrm{Cys}_{2}\right)$. Naive T cells must acquire L-Cys from APCs because they lack the cystine transporter $\mathrm{x}_{c}{ }^{-}$and therefore cannot import L-Cys 2 , and cannot de novo synthesize L-Cys because they lack cystathionase, the enzyme that converts methionine to L-Cys. MDSCs also lack cystathionase and therefore must scavenge L-Cys 2 . Since MDSCs do not export L-Cys due to their lack of the neutral amino-acid alanine-serine-cysteine transporter 1 (ASC), high levels of MDSCs quickly deplete their local environment of $\mathrm{L}-\mathrm{Cys}_{2}$, thereby limiting the ability of APCs to provide T cells with L-Cys. The role of MDSCs and their biological relevance in L-Cys depletion is supported by the correlation between high levels of MDSCs and reduced serum $\mathrm{L}-\mathrm{Cys}_{2}$ in tumor-bearing mice (Srivastava, Sinha, Clements, Rodriguez, \& Ostrand-Rosenberg, 2010). Since activated T cells express $\mathrm{x}_{c}{ }^{-}$, theoretically they should be resistant to this suppressive mechanism (Levring et al., 2012). However, since ARG1 production by MDSCs suppresses T cell activation, it is unclear if T cell upregulation of $\mathrm{x}_{c}{ }^{-}$is functionally relevant.

\subsection{MDSC Production of NO}

NOS also catabolizes L-Arg and contributes to MDSC-mediated immune suppression (Bronte \& Zanovello, 2005; Raber et al., 2014). MDSCs produce NO by the action of NOS2 and NOS3. PMN-MDSCs are NOS $2^{\text {low }} \mathrm{NOS}^{\text {hi }}$, while MO-MDSCs are $\mathrm{NOS} 2^{\text {hi }} \mathrm{NOS}^{\text {low }}$ (Raber et al., 2014). NOS 2 generates more NO than NOS 3 and is induced by pro-inflammatory cytokines, endotoxin, hypoxia, and oxidative stress, while NOS3 is constitutively expressed (Fukumura, Kashiwagi, \& Jain, 2006). 
NO is labile and reacts with multiple compounds to produce many toxic and regulatory factors. For example, NO reacts with (i) cysteine thiol groups on proteins and peptides, which form S-nitrosothiols, thereby altering a protein's tertiary structure; (ii) superoxide anions $\left(\mathrm{O}_{2}{ }^{-}\right)$, which form peroxynitrite (PNT, $\mathrm{ONOO}^{-}$), a molecule that alters protein structure; (iii) divalent cations (e.g., $\mathrm{Fe}^{2+}$ and $\mathrm{Zn}^{2+}$ ), which regulate the function of various transcription factors and enzymes; (iv) nucleic acids, which cause mutagenesis; and (v) unsaturated lipids, which lead to the formation of nitrolipids that can have pro- or anti-inflammatory activity (Bogdan, 2015). Since NO influences many biological processes, it is not surprising that NO is capable of pro- and antitumor activity. NO can induce tumor-cell apoptosis and inhibit metastasis, or enhance tumor-cell invasion, proliferation, and angiogenesis (Fukumura et al., 2006). However, MDSC-produced NO negatively impacts T cells. NO inhibits JAK3, STAT5, ERK, and AKT, which prevents IL-2 signaling, thereby impairing the generation of effector and memory $\mathrm{T}$ cells (Mazzoni et al, 2002). NO directly inhibits these signaling proteins by S-nitrosothiolation, or indirectly by activating guanylate cyclase and cyclic-GMP-dependent kinases (Serafini, 2013). S-nitrosothiolation of ARG1 enhances ARG1 affinity for L-Arg which subsequently increases $\mathrm{ARG} 1$ activity, thereby establishing a synergistic relationship between ARG1- and NO-mediated immune suppression (Santhanam et al., 2007).

\subsection{MDSC Production of ROS}

NOX is a membrane-bound enzyme complex that is utilized by MDSCs to suppress $\mathrm{T}$ cell activation. MDSCs from tumor-bearing mice have enhanced expression of the NOX subunits gp91, p22, and p47 and produce more ROS than MDSCs from tumor-free mice (Corzo et al., 2009). NOX generates superoxide which spontaneously reacts with many molecules to produce a variety of ROS including $\mathrm{H}_{2} \mathrm{O}_{2}$, hydroxyl radical, and hypochlorous acid. These ROS damage proteins, lipids, and nucleic acids, thereby enhancing inflammation and promoting apoptosis. For example, $\mathrm{H}_{2} \mathrm{O}_{2}$ production in cancer patients reduces $\mathrm{T}$ cell production of cytokines and expression of

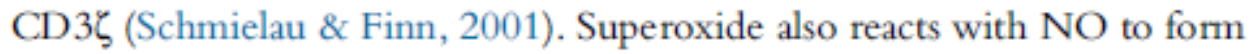
PNT, which is produced by PMN-MDSCs through the action of gp91 and NOS3 (Raber et al., 2014). PNT nitrates/nitrosylates the TCR and MHC (Lu \& Gabrilovich, 2012), thereby disrupting TCR-MHC I/peptide 
binding and rendering tumor cells resistant to CTL-mediated apoptosis (Lu et al., 2011). Due to the short half-life of PNT, these reactions are limited to short distances and require close cell-to-cell contact. PNT also reacts with the chemoattractant CCL2, thereby inhibiting $\mathrm{T}$ cell infiltration into tumors (Molon et al., 2011).

\subsection{MDSCs Inhibit T Cell Migration by Downregulating L- and E-Selectins}

Activation of tumor-reactive $\mathrm{T}$ cells requires entry of naive $\mathrm{T}$ cells into tumor-draining lymph nodes or migration to the TME. L-selectin mediates the first step in extravasation by facilitating $\mathrm{T}$ cell adhesion to high endothelial venules (HEVs). Naive T cells with low expression of $\mathrm{L}$-selectin do not adhere efficiently to HEVs and fail to enter lymph nodes (J. Mihich, S. Evans, S. Abrams, \& S. Ostrand-Rosenberg, unpublished data). In tumor-bearing mice, MDSCs prevent $\mathrm{T}$ cell entry into lymph nodes by downregulating L-selection through their extracellular expression of ADAM17, the enzyme that cleaves L-selectin on naïve $\mathrm{T}$ cells (Hanson, Clements, Sinha, Ilkovitch, \& Ostrand-Rosenberg, 2009; Parker et al., 2014).

In squamous cell carcinoma patients, MDSCs also prevent the homing of T cells to tumor sites by downregulating E-selectin on tumor vessels. In order for T cells to adhere to tumor vessels and subsequently enter the tumor mass, they must first bind to E-selectin. However, NO produced by MDSCs decreases E-selectin levels, thereby limiting $\mathrm{T}$ cell access to tumor (Gehad et al., 2012).

\subsection{MDSCs Express Programmed Death-Ligand 1}

Tumor cells escape antitumor immunity through their expression of programmed death-ligand 1 (PD-L1). When PD-L1 binds to its receptor PD-1 on $\mathrm{T}$ cells, it induces $\mathrm{T}$ cell exhaustion/apoptosis. MDSCs from some tumor-bearing mice and patients express PD-L1 (Youn, Nagaraj, Collazo, \& Gabrilovich, 2008; Zhang, Wang, et al., 2013). Some tumorinfiltrating MDSCs have elevated expression of PD-L1 due to hypoxiainduced upregulation of HIF- $1 \alpha$ (Noman et al., 2014). However, MDSCs do not universally express PD-L1, and PD-L1 blockade does not always decrease MDSC suppressive activity (Youn et al., 2008). 


\subsection{MDSCs Induce Tregs and Th17 Cells}

Tregs play an important role in the control of immune reactivity against selfand non-self-antigens, and in some animal models, they protect tumors from antitumor immunity. Tregs are characterized as $\mathrm{CD} 4^{+} \mathrm{FoxP} 3^{+}$cells. MDSCs induce/expand Tregs in vitro and in vivo in multiple tumor models (Adeegbe et al., 2011; Huang et al., 2006; MacDonald et al., 2005; Zoso et al., 2014). MDSCs induce Tregs by secreting IL-10 and TGF $\beta$ (Hoechst et al., 2008; Huang et al., 2006) and activate Tregs by presenting tumor-specific antigens in an ARG-dependent and TGF $\beta$-independent manner (Serafini, Mgebroff, Noonan, \& Borrello, 2008). MDSC expression of CD40 is required for MDSC-mediated Treg induction, since CD40-deficient MDSCs do not drive Treg expansion (Pan et al., 2010). Given the link between MDSCs and Tregs, therapies targeting MDSCs may also reduce Treg populations.

Th17 cells are a pro-inflammatory $\mathrm{CD}^{+} \mathrm{T}$ cell subset $\left(\mathrm{CD} 4^{+} \mathrm{ROR} \gamma \mathrm{t}^{+} \mathrm{IL}-17^{+}\right)$. Since they have both pro- and antitumor effects, their role in antitumor immunity is controversial (Ye, Livergood, \& Peng, 2013). MDSCs induce Th17 cells by producing IL-6 and TGF $\beta$ (Chatterjee et al., 2013). IFN $\gamma$ - or TNF $\alpha$-activated MDSCs also recruit Th17 cells through their production of CCL4, which is a Th17 chemoattractant (Ortiz et al., 2015). As previously mentioned, IL-17 drives the accumulation of MDSCs. Therefore, MDSCs and Th17 cells may induce each other.

\subsection{MDSCs Impair NK Cell-Mediated Cytotoxicity}

MDSCs impair NK function via contact-dependent mechanisms. MDSCs produce TGF $\beta$ and $\mathrm{H}_{2} \mathrm{O}_{2}$ which decrease NK cell expression of the activating receptors NKG2D, NKp46, and NKp44, thereby making NK cells more difficult to activate (Elkabets et al., 2010; Mao et al., 2014). MDSCs also decrease the ability of NK cells to induce apoptosis by downregulating NK cell production of perforin which is essential for NK-mediated target cell lysis. In addition, MDSCs suppress NK cells by limiting their response to IL-2, a growth factor that enhances NK cell proliferation and cytolytic activity (Liu et al., 2007).

\subsection{Cross Talk Between MDSCs, Macrophages, Tumor Cells, and MCs Enhances Inflammation and Promotes MDSC Suppressive Activity}

Solid tumors are a complex and frequently inflamed microenvironment. Both tumor and host (macrophages, DCs, MCs, MDSCs, and fibroblasts) cells 
within solid tumors participate in cross talk that regulates the release of proand anti-inflammatory cytokines and drive the accumulation and suppressive function of immune-suppressive cells such as Tregs, TAMs, and MDSCs.

Macrophages can be either tumoricidal (M1-like) or tumor-promoting (M2-like) (Sica \& Mantovani, 2012). MDSCs subvert macrophages toward an M2 phenotype through their production of $\mathrm{IL}-10$ which downregulates macrophage production of IL-12 and TNF $\alpha$, while simultaneously enhancing macrophage production of NO (Beury et al., 2014; Sinha, Clements, Bunt, Albelda, \& Ostrand-Rosenberg, 2007). IL-12 downregulation is mediated by both intact MDSCs and MDSC-derived exosomes (Burke, Choksawangkarn, Edwards, Ostrand-Rosenberg, \& Fenselau, 2014). MDSC production of IL-10 involves TLR 4 signaling and is increased by inflammation and direct cell-to-cell contact with macrophages (Bunt, Clements, Hanson, Sinha, \& Ostrand-Rosenberg, 2009; Sinha, Clements, Bunt, et al., 2007), and via the adenosine A2A receptor (Cekic, Day, Sag, \& Linden, 2014). MDSCs also decrease macrophage expression of MHC II through both IL-10-dependent and -independent mechanisms (P. Sinha, D. Beury, V. Clements, \& S. Ostrand-Rosenberg, unpublished) and upregulate PD-L1 on macrophages in the liver (Ilkovitch \& Lopez, 2009).

MDSCs and tumor cells also participate in cross talk. Tumor cells increase MDSC production of $\mathrm{IL}-6$, and in turn, MDSCs enhance tumor-cell production of IL-6. IL-6 also increases MDSC suppressive activity, but inhibits MDSC production of IL-10 (Beury et al., 2014). In addition, tumor cells enhance MDSC production of IL-28, which facilitates tumorcell invasion, migration, and angiogenesis (Mucha, Majchrzak, Taciak, Hellmen, \& Krol, 2014).

MCs and MDSCs also interact. MDSC and MC cross talk drives inflammation by increasing production of TNF $\alpha$, CCL3, IL-4, IL-13, IL-6, and CCL2 (Danelli et al., 2015; Martin et al., 2014; Saleem et al., 2012). The latter two molecules are regulated by ligation of MC CD40L to CD40 on MDSCs. Activated MCs release histamine which signals through histamine receptors 1,2, and 3 on MDSCs and enhances MDSC expression of $\mathrm{IL}-4$ and IL-13. Histamine upregulates ARG1 and NOS2 in MO-MDSCs and decreases ARG1 and NOS2 in PMN-MDSCs (Martin et al., 2014). Since histamine increases MO-MDSC production of NO and downregulates immune-suppressive mediators of PMN-MDSC, the net effect of histamine is to increase MO-MDSC suppressive activity (Danelli et al., 2015). 


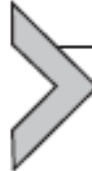

\section{MDSCS IN NONCANCER SETTINGS}

MDSCs are also elevated in noncancer settings, where they can be either detrimental or beneficial. For example, elevated levels of MDSC decrease immune responsiveness in patients with toxoplasmosis (Voisin, Buzoni-Gatel, Bout, \& Velge-Roussel, 2004) and trypanosomiasis (Goñi, Alcaide, \& Fresno, 2002). MDSCs are also elevated in mice with antigen-induced autoimmune enterocolitis, where adoptive transfer of additional MDSCs reduces disease symptoms, suggesting a protective role for MDSCs (Haile et al., 2008). Likewise, mice with experimental autoimmune encephalomyelitis have elevated levels of immune-suppressive MDSCs in their spleens and blood, which are likely to be beneficial in limiting autoreactivity (Zhu et al., 2007). Elevated levels of MDSCs are also found in the serum of patients with sepsis where they polarize immunity toward an antibody-promoting Type 2 response (Delano et al., 2007). Whether the MDSCs are beneficial or detrimental in sepsis is unclear.

Both stress and aging are also associated with increased MDSC levels. For example, postsurgery traumatic stress in mice is accompanied by increased levels of splenic MDSCs that suppress $T$ cell proliferation by an ARG1-dependent mechanism (Makarenkova, Bansal, Matta, Perez, \& Ochoa, 2006). Psychological stress in breast cancer patients further elevates circulating MDSCs (Mundy-Bosse, Thornton, Yang, Andersen, \& Carson, 2011). MDSCs also increase with aging as shown in a study of adults ages 19-59, 61-76 (seniors), and 67-99 (elderly). The elderly cohort had the highest levels of MDSCs as well as increased serum levels of IL-6 and IL-1 $\beta$ (Verschoor et al., 2013). Studies in aging mice similarly show increases in MDSCs (Grizzle et al., 2007; Hanson et al., 2009).

MDSCs have also been implicated in driving asthma, an allergy caused by a hyper Th2 response that disrupts the normal Th1/Th2 balance. Children with asthma have elevated serum levels of MDSCs and IL-10, and reduced levels of IL-12 (Zhang, Luan, et al., 2013). Since MDSCs produce IL-10 which decreases macrophage production of IL-12 (Sinha, Clements, Bunt, et al., 2007), MDSCs are likely increasing the severity of disease by exacerbating polarization toward a type 2 response. In contrast, in a mouse asthma model, MDSCs appear to reduce disease because injection of tumor-derived MDSCs 
restored the Th1/Th2 balance by reducing the type 2 cytokine IL-4 and increasing the type 1 cytokine IFN- $\boldsymbol{\gamma}$ (Song et al., 2014).

MDSCs may play a beneficial role in obesity, which is considered a chronic low-grade inflammatory disease. Obese individuals have elevated levels of MDSCs in their peripheral tissues. These MDSCs counterbalance some of the detrimental effects of obesity by promoting insulin sensitivity and reducing inflammation. This latter effect occurs because MDSCs in adipose tissue skew macrophages toward an anti-inflammatory M2 phenotype (Xia et al., 2011), possibly by their production of IL-10. However, MDSCs may be detrimental in obese individuals undergoing vaccination, since mice with diet-induced obesity and elevated levels of MDSC displayed decreased antigen-specific T cell responses following vaccination (Chen et al., 2015).

Because of their immunosuppressive potency, MDSCs have been tested as therapeutic agents for autoimmune diseases or when tolerance is required. For example, adoptive transfer of bone marrow-generated MDSCs has been used to combat graft-versus-host disease (Highfill et al., 2010), ameliorate experimental autoimmune encephalomyelitis (Ioannou et al., 2012), aid in the retention of allogeneic islet grafts (Chou et al., 2012), and induce Tregs to protect against type 1 diabetes (Yin et al., 2010). MDSCs may also be needed in mice to maintain maternal-fetal tolerance during the development of allogeneic fetuses (P. Sinha \& S. Ostrand-Rosenberg, unpublished). The presence of elevated levels of immune-suppressive MDSCs in both mice and women pregnant with allogeneic embryos further supports the concept that MDSCs contribute to maternal-fetal tolerance and suggests that reduced levels of MDSCs in pregnant women could lead to miscarriage (Köstlin et al., 2014).

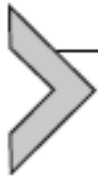

\section{THERAPEUTIC TARGETING OF MDSCS}

Because of their central role in immune suppression, many investigators have focused on neutralizing MDSCs in individuals with cancer. Strategies include targeting MDSC suppressive mechanisms, inducing MDSCs to differentiate into nonsuppressive mature APCs, blocking development of MDSCs, and killing of MDSCs. Table 1 lists recent approaches. Older therapies are reviewed in Wesolowski, Markowitz, and Carson (2013). It should be noted that none of these approaches universally neutralize MDSCs. 
Table 1 Recently Reported Experimental Therapies Targeting MDSC Development, Viability, or Function

\begin{tabular}{|c|c|c|c|}
\hline Therapy & Mechanism & Model & Effect \\
\hline $\begin{array}{l}{ }^{\mathrm{a}} 2 \mathrm{aG} 4 \text { (phosphatidyl- } \\
\text { serine antibody) }\end{array}$ & $\begin{array}{l}\text { Reverses immunosuppressive } \\
\text { effects by phosphatidyl-serine }\end{array}$ & $\begin{array}{l}\text { LNCaP and PC3 prostate cancers } \\
\text { (SCID mice) }\end{array}$ & $\begin{array}{l}\text { Induces MDSC differentiation into } \\
\text { M1 macrophages and DC; reduces } \\
\text { MDSC numbers in tumor }\end{array}$ \\
\hline $\begin{array}{l}\text { b5-AZA (5- } \\
\text { azacytidine) }\end{array}$ & Inhibits DNA methyltransferase & $\begin{array}{l}\text { TRAMP-C } 2 \text { prostate adenoma } \\
\text { and TC-1/A } 9 \text { pancreatic adenoma } \\
\text { (C57BL/6 mice) }\end{array}$ & $\begin{array}{l}\text { Reduces MDSC ARG1 expression, } \\
\text { VEGF production, and suppressive } \\
\text { activity; reduces MDSC } \\
\text { accumulation. }\end{array}$ \\
\hline $\begin{array}{l}{ }^{\mathrm{c}} 5-\mathrm{AZA}+\mathrm{ENT} \\
\text { (entinostat) }\end{array}$ & ENT is a class I HDAC inhibitor & $\begin{array}{l}\text { CT26 colon carcinoma and } 4 \mathrm{~T} 1 \\
\text { mammary carcinoma (BALB/c } \\
\text { mice) }\end{array}$ & $\begin{array}{l}\text { ENT causes apoptosis of PMN- } \\
\text { MSDC in vitro, while 5-AZA has no } \\
\text { effect on MDSCs; causes rejection of } \\
\text { tumor when 5-AZA + ENT is used in } \\
\text { combination with } \alpha \text { PD- } 1+\alpha C \text { CTA- } 4 \\
\text { immunotherapy }\end{array}$ \\
\hline${ }^{\mathrm{d}}$ ABT-737 & $\begin{array}{l}\text { Inhibitor of } \mathrm{Bcl}-2, \mathrm{Bcl}-\mathrm{xL} \text {, and } \\
\text { Bcl-w }\end{array}$ & $\begin{array}{l}\text { CT26 colon carcinoma and } 4 \mathrm{~T} 1 \\
\text { mammary carcinoma (BALB/c } \\
\text { mice) }\end{array}$ & $\begin{array}{l}\text { Increases MDSC susceptibility to } \\
\text { FASL-mediated apoptosis; increases } \\
\text { apoptosis of MDSC in vivo, not in vitro; } \\
\text { decreases MDSC accumulation }\end{array}$ \\
\hline${ }^{\mathrm{e}}$ Antaxinib & VEGFR antagonist & $\begin{array}{l}\text { RENCA renal cell carcinoma } \\
\text { (BALB/c mice) }\end{array}$ & $\begin{array}{l}\text { Inhibits STAT3 in MDSC; decreases } \\
\text { MDSC ROS and ARG1; increases } \\
\text { MDSC apoptosis }\end{array}$ \\
\hline $\begin{array}{l}{ }^{\mathrm{f}} \mathrm{ATRA} \text { (all-trans } \\
\text { retinoic acid) }\end{array}$ & Agonist for retinoic acid receptor & SCLC patients & $\begin{array}{l}\text { Causes apoptosis of PMN-MDSCs; } \\
\text { differentiates MO-MDSCs to } \\
\text { macrophages and DCs; reduces } \\
\text { MDSCs in SCLC patients; enhanced } \\
\text { the number of responders to p53 } \\
\text { vaccine }\end{array}$ \\
\hline
\end{tabular}

\begin{tabular}{|c|c|c|c|}
\hline${ }^{\mathrm{g}} \mathrm{CD} 16 \mathrm{XCD} 33 \mathrm{BiKE}$ & Targets NK cells to $\mathrm{CD} 33^{+}$cells & $\begin{array}{l}\text { Myelodysplastic syndrome (MDS) } \\
\text { patients }\end{array}$ & $\begin{array}{l}\text { Induces NK cell-mediated killing of } \\
\text { MDSCs }\end{array}$ \\
\hline $\begin{array}{l}{ }^{\mathrm{h}} \text { Corosolic acid } \\
\text { (Triterpenoid from } \\
\text { apple pomace) }\end{array}$ & $\begin{array}{l}\text { Blocks activation of STAT3 and } \\
\text { NF- } \kappa \text { B; inhibits polarization of } \\
\text { macrophages to M } 2 \text { phenotype }\end{array}$ & LM85 osteosarcoma (C3H mice) & Reduces MDSC suppressive activity \\
\hline${ }^{\mathrm{i}}$ Dopamine & $\begin{array}{l}\text { Signals through D1-like DA } \\
\text { receptors which inhibited } \\
\text { MO-MDSC decreasing NO }\end{array}$ & $\begin{array}{l}\text { LLC and B16 melanoma (C57BL/ } \\
6 \text { mice) }\end{array}$ & Reduces MDSC suppressive activity \\
\hline $\begin{array}{l}{ }^{\mathrm{j}} \text { Gemcitabine }+ \\
\text { Capecitabine }\end{array}$ & $\begin{array}{l}\text { Gemcitabine is a nucleoside } \\
\text { analog. Capecitabine is a prodrug } \\
\text { that is enzymatically converted to } \\
\text { fluorouracil }\end{array}$ & Pancreatic cancer patients & $\begin{array}{l}\text { No direct effect on MDSCs alone, but } \\
\text { reduces MDSCs in patients receiving } \\
\text { GM-CSF as an adjuvant for GV1001 } \\
\text { (GV1001 is a telomerase vaccine) }\end{array}$ \\
\hline $\begin{array}{l}{ }^{\mathrm{k}} \text { Gemcitabine } \\
\text { + Rosiglitazone }\end{array}$ & $\begin{array}{l}\text { Rosiglitazone activates PPAR } \gamma \text {, } \\
\text { thereby acting as an anti- } \\
\text { inflammatory agent }\end{array}$ & $\begin{array}{l}\text { Panc02 pancreatic carcinoma } \\
\text { (C57BL/6 mice) }\end{array}$ & $\begin{array}{l}\text { Rosiglitazone reduces early MDSC } \\
\text { accumulation; combination therapy } \\
\text { reduces late-stage MDSC } \\
\text { accumulation }\end{array}$ \\
\hline $\mathrm{c} J 32$ & PI3K inhibitor & $\begin{array}{l}\text { 4T1 mammary carcinoma } \\
\text { (BALB/c mice) }\end{array}$ & $\begin{array}{l}\text { Causes PMN-MDSC apoptosis; no } \\
\text { effect on tumor growth alone; causes } \\
\text { tumor rejection in mice when used in } \\
\text { combination with } \alpha \mathrm{PD}-1+\alpha \text { CTLA- } 4 \\
\text { immunotherapy }\end{array}$ \\
\hline $\begin{array}{l}{ }^{1} \text { Lenalidomide } \\
\text { (thalidomide } \\
\text { derivative) }\end{array}$ & $\begin{array}{l}\text { Inhibits NF- } \kappa \mathrm{B}, \mathrm{COX}-2 \text { activity, } \\
\text { and angiogenesis; has } \\
\text { immunomodulatory effects }\end{array}$ & A20 lymphoma (BALB/c mice) & $\begin{array}{l}\text { Reduces MDSCs in vivo, but does not } \\
\text { alter MDSC levels in naïve mice }\end{array}$ \\
\hline
\end{tabular}


Table 1 Recently Reported Experimental Therapies Targeting MDSC Development, Viability, or Function-cont'd

Therapy Mechanism Model Effect

\begin{tabular}{|c|c|c|c|}
\hline${ }^{\mathrm{m}} \mathrm{MI}-319$ & HDM2 inhibitor & $\begin{array}{l}\text { Human renal cell carcinoma (nude } \\
\text { mice) }\end{array}$ & $\begin{array}{l}\text { Reverses sunitinib-induced MDSC } \\
\text { infiltration into tumor (sunitinib is a } \\
\text { RTK inhibitor and reduces } \\
\text { angiogenesis) }\end{array}$ \\
\hline $\begin{array}{l}{ }^{\mathrm{n}} \text { Polyphenon } \\
\mathrm{E} \text { (green tea extract) }\end{array}$ & Unknown mechanism & $\begin{array}{l}\text { Neuroblastoma mouse models: } \\
\text { TH-MYCN transgenic mice, } \\
\text { human SHSY } 5 Y \text { (NOD/SCID } \\
\text { mice), Neuro } 2 \mathrm{~A} \text { (A/J mice) }\end{array}$ & $\begin{array}{l}\text { Differentiates MO-MDSCs into } \\
\text { PMN-MDSCs; reduces suppressive } \\
\text { activity of MO-MDSCs; decreases } \\
\text { ARG1 in MDSCs }\end{array}$ \\
\hline${ }^{\circ}$ SAR 131675 & Inhibits VEGFR-3 tyrosine kinase & $\begin{array}{l}\text { 4T1 mammary carcinoma } \\
\text { (BALB/c mice) }\end{array}$ & $\begin{array}{l}\text { Reduces the number of MDSCs in } \\
\text { tumor; promotes M1 macrophages }\end{array}$ \\
\hline${ }^{\mathrm{P}}$ Silibinin & Anti-inflammatory flavonoid & $\begin{array}{l}\text { 4T1 mammary carcinoma } \\
\text { (BALB/c mice) }\end{array}$ & $\begin{array}{l}\text { Reduces tumor volume, increases } \\
\text { survival of tumor-bearing mice; } \\
\text { decreases total number of MDSCs }\end{array}$ \\
\hline${ }^{\mathrm{q}}$ Tadalafil & PDE5 inhibitor & Human HNSCC patients & $\begin{array}{l}\text { Lowers MDSCs and Treg numbers; } \\
\text { increases tumor-specific CD }{ }^{+} \mathrm{T} \text { cells } \\
\text { in a dose-dependent manner }\end{array}$ \\
\hline $\begin{array}{l}{ }^{\mathrm{I}} \mathrm{TCBA} \\
\text { (tetrabromocinnamic } \\
\text { acid) }\end{array}$ & Restores Notch signaling & $\begin{array}{l}\text { EL4 lymphoma (C57BL/6 mice), } \\
\text { CT26 colon carcinoma and } \\
\text { MethA sarcoma (BALB/c mice) }\end{array}$ & $\begin{array}{l}\text { Induces differentiation of MDSCs to } \\
\text { DCs }\end{array}$ \\
\hline${ }^{s}$ Vemurafenib & $\begin{array}{l}\text { Inhibitor of } \mathrm{B}-\mathrm{RAF}^{\mathrm{V} 600 \mathrm{E}} \text {, a } \\
\text { mutation leading to constitutive } \\
\text { activation of MAP kinase pathway }\end{array}$ & Cutaneous melanoma patients & $\begin{array}{l}\text { Inhibits the release of soluble factors } \\
\text { from melanoma cells involved in the } \\
\text { generation of MO-MDSC in vitro; } \\
\text { decreases MO-MDSC in vivo }\end{array}$ \\
\hline
\end{tabular}

${ }^{\mathrm{t}}$ Withaferin A (extract Antioxidant with antitumor from ashwagandha effects; inhibits Notch signaling plant)
4T1 mammary carcinoma (BALB/c mice)
Decreases MDSC production of ROS and IL-10; decreases MDSC suppressive activity; reduces MDSC accumulation

${ }^{2}$ Yin, Huang, Lynn, and Thorpe (2013).

${ }^{\mathrm{b}}$ Mikyskova et al. (2014).

${ }^{\mathrm{C}} \mathrm{Kim}$ et al. (2014).

${ }^{\mathrm{d}} \mathrm{Hu}$ et al. (2013).

eYuan et al. (2014).

${ }^{f}$ Iclozan, Antonia, Chiappori, Chen, and Gabrilovich (2013).

${ }^{\mathrm{g}}$ Gleason et al. (2014).

${ }^{\text {h}}$ Horlad et al. (2013)

${ }^{\mathrm{i}} \mathrm{Wu}$ et al. (2015).

${ }^{\mathrm{j}}$ Annels et al. (2014).

${ }^{k}$ Bunt, Mohr, Bailey, Grandgenett, and Hollingsworth (2013).

${ }^{1}$ Sakamaki et al. (2014).

mPanka, Liu, Geissler, and Mier (2013).

${ }^{\text {n}}$ Santilli et al. (2013).

${ }^{\circ}$ Espagnolle et al. (2014).

${ }^{\mathrm{P}}$ Forghani, Khorramizadeh, and Waller (2014).

${ }^{\mathrm{q}}$ Weed et al. (2015)

${ }^{\mathrm{r}}$ Cheng et al. (2014).

sSchilling et al. (2013).

'Sinha and Ostrand-Rosenberg (2013). 


\section{CONCLUSIONS}

MDSCs encompass a range of immature immune-suppressive myeloid cells. Their suppressive activity and accumulation are induced by many inflammatory mediators with unique and redundant signaling pathways. MDSCs inhibit antitumor immunity through several mechanisms including (i) depletion of the local environment of the amino acids L-Arg, L-Trp, and L-Cys, which inhibits T cell activation and/or proliferation; (ii) secretion of NO, PNT, and ROS, which causes T cell apoptosis, inhibits peptide recognition by T cells, and inhibits T cell activation; (iii) induction of immunosuppressive Tregs; and (iv) impairment of $\mathrm{T}$ cell trafficking to lymph nodes. MDSCs also alter the inflammatory milieu by inducing inflammatory Th17 cells, participating in cross talk with macrophages, tumor cells, and MCs which promotes a protumor environment that enhances tumor-cell growth, invasion, and metastasis. Therefore, neutralizing MDSCs is an obvious strategy to enhance natural antitumor immunity and boost the efficacy of immunotherapies.

The concept of activating a patient's immune system to destroy their endogenous cancer cells has been a goal of immunotherapy for many years. Unfortunately, many cancer immunotherapy clinical trials have failed to show therapeutic efficacy. MDSCs may be responsible for at least some of these failures since they are present in many cancer patients, and have the ability to prevent $\mathrm{T}$ cell activation. Analysis of blood samples from nonresponder patients indicated a correlation between lack of response and MDSC levels (Kimura et al., 2013). Regardless of the outcome of such studies, it is likely that cancer immunotherapies involving in vivo activation or proliferation of tumor-reactive $\mathrm{T}$ cells will require adjunctive treatment that neutralizes MDSCs.

Accumulation and suppressive potency of MDSCs are regulated by a complex milieu of inflammatory mediators. Environmental conditions such as hypoxia and inflammation act through similar signaling networks. These networks converge on common transcription factors such as STAT3 and $\mathrm{NF}-\kappa \mathrm{B}$ and regulate additional transcription factors, miRNAs, and proteins that mediate MDSC accumulation and suppression. Since multiple ligands initiate signaling through these pathways, MDSC regulation is highly redundant. This redundancy allows for the development of MDSCs under a broad range of conditions and may explain why MDSCs are so widespread in cancer patients. The redundancy also complicates therapeutic approaches for neutralizing MDSCs, since different inducers compensate for each other. 
The past decade has seen remarkable progress in recognizing MDSCs as key players that inhibit antitumor immunity and facilitate tumor progression. Advances in understanding the mechanisms that drive MDSC accumulation and function have also been extensive. Hopefully, these studies will lead to the development of therapeutic strategies that are universally effective in neutralizing or eliminating MDSCs in cancer patients.

\section{REFERENCES}

Adeegbe, D., Serafini, P., Bronte, V., Zoso, A., Ricordi, C., \& Inverardi, L. (2011). In vivo induction of myeloid suppressor cells and CD4(+)Foxp3(+) T regulatory cells prolongs skin allograft survival in mice. Cell Transplantation, 20, 941-954.

Alizadeh, D., Trad, M., Hanke, N. T., Larmonier, C. B., Janikashvili, N., Bonnotte, B., et al. (2014). Doxorubicin eliminates myeloid-derived suppressor cells and enhances the efficacy of adoptive T-cell transfer in breast cancer. Cancer Researdh, 74, 104-118.

Almand, B., Clark, J. I., Nikitina, E., van Beynen, J., English, N. R., Knight, S. C., et al. (2001). Increased production of immature myeloid cells in cancer patients: A mechanism of immunosuppression in cancer. Joumal of Immunology, 166, 678-689.

Annels, N. E., Shaw, V. E., Gabitass, R. F., Billingham, L., Corrie, P., Eatock, M., et al. (2014). The effects of gemcitabine and capecitabine combination chemotherapy and of low-dose adjuvant GM-CSF on the levels of myeloid-derived suppressor cells in patients with advanced pancreatic cancer. Cancer Immunology, Immunotherapy, 63, $175-183$.

Balkwill, F., \& Mantovani, A. (2001). Inflammation and cancer: Back to Virchow? Lancet, $357,539-545$.

Bergers, G., Brekken, R., McMahon, G., Vu, T. H., Itoh, T., Tamaki, K., et al. (2000). Matrix metalloproteinase-9 triggers the angiogenic switch during carcinogenesis. Nature Cell Biology, 2, 737-744.

Beury, D. W., Parker, K. H., Nyandjo, M., Sinha, P., Carter, K. A., \& Ostrand-Rosenberg, S. (2014). Cross-talk among myeloid-derived suppressor cells, macrophages, and tumor cells impacts the inflammatory milieu of solid tumors. Journal of Leukocyte Biology, 96, $1109-1118$.

Bianchi, M. E., \& Manfredi, A. A. (2007). High-mobility group box 1 (HMGB1) protein at the crossroads between innate and adaptive immunity. Immunological Reviews, 220, $35-46$.

Bogdan, C. (2015). Nitric oxide synthase in innate and adaptive immunity: An update. Trends in Immunology, 36, 161-178.

Boldin, M. P., Taganov, K. D., Rao, D. S., Yang, L., Zhao, J. L., Kalwani, M., et al. (2011). miR-146a is a significant brake on autoimmunity, myeloproliferation, and cancer in mice. The Joumal of Experimental Mediane, 208, 1189-1201.

Brandau, S., Trellakis, S., Bruderek, K., Schmaltz, D., Steller, G., Elian, M., et al. (2011). Myeloid-derived suppressor cells in the peripheral blood of cancer patients contain a subset of immature neutrophils with impaired migratory properties. Journal of Leukocyte Biology, 89, 311-317.

Bresnick, A. R., Weber, D. J., \& Zimmer, D. B. (2015). S100 proteins in cancer. Nature Revieus. Cancer, 15, 96-109.

Brimnes, M. K., Vangsted, A. J., Knudsen, L. M., Gimsing, P., Gang, A. O., Johnsen, H. E., et al. (2010). Increased level of both CD4+FOXP3 + regulatory $\mathrm{T}$ cells and CD14+HLA-DR ${ }^{-}$/low myeloid-derived suppressor cells and decreased level of dendritic cells in patients with multiple myeloma. Scandinavian Journal of Immunology, 72, 540-547. 
Bronte, V., Chappell, D. B., Apolloni, E., Cabrelle, A., Wang, M., Hwu, P., et al. (1999). Unopposed production of granul ocyte-macrophage colony-stimulating factor by tumors inhibits CD8 $+\mathrm{T}$ cell responses by dysregulating antigen-presenting cell maturation. Journal of Immunology, 162, 5728-5737.

Bronte, V., Serafini, P., De Santo, C., Marigo, I., Tosello, V., Mazzoni, A., et al. (2003). IL-4-induced arginase 1 suppresses alloreactive $\mathrm{T}$ cells in tumor-bearing mice. Joumal of Immunology, 170, 270-278.

Bronte, V., \& Zanovello, P. (2005). Regulation of immune responses by L-arginine metabolism. Nature Revieus. Immunology, 5, 641-654.

Bunt, S. K., Clements, V. K., Hanson, E. M., Sinha, P., \& Ostrand-Rosenberg, S. (2009). Inflammation enhances myeloid-derived suppressor cell cross-talk by signaling through Toll-like receptor 4. Journal of Leukocyte Biology, 85, 996-1004.

Bunt, S. K., Mohr, A. M., Bailey, J. M., Grandgenett, P. M., \& Hollingsworth, M. A. (2013). Rosiglitazone and Gemcitabine in combination reduces immune suppression and modulates T cell populations in pancreatic cancer. Cancer Immunology, Immunotherapy, 62, $225-236$.

Bunt, S. K., Sinha, P., Clements, V. K., Leips, J., \& Ostrand-Rosenberg, S. (2006). Inflammation induces myeloid-derived suppressor cells that facilitate tumor progression. Joumal of Immunology, 176, 284-290.

Bunt, S. K., Yang, L., Sinha, P., Clements, V. K., Leips, J., \& Ostrand-Rosenberg, S. (2007). Reduced inflammation in the tumor microenvironment delays the accumulation of myeloid-derived suppressor cells and limits tumor progression. Cancer Research, 67, 10019-10026.

Burchert, A., Cai, D., Hofbauer, L. C., Samuelsson, M. K., Slater, E. P., Duyster, J., et al. (2004). Interferon consensus sequence binding protein (ICSBP; IRF-8) antagonizes BCR/ABL and down-regulates bcl-2. Blood, 103, 3480-3489.

Burke, M., Choksawangkam, W., Edwards, N., Ostrand-Rosenberg, S., \& Fenselau, C. (2014). Exosomes from myeloid-derived suppressor cells carry biologically active proteins. Journal of Proteome Reseanh, 13, 836-843.

Cekic, C., Day, Y. J., Sag, D., \& Linden, J. (2014). Myeloid expression of adenosine A2A receptor suppresses $\mathrm{T}$ and $\mathrm{NK}$ cell responses in the solid tumor microenvironment. Canaer Reseanch, 74, 7250-7259.

Chalmin, F., Ladoire, S., Mignot, G., Vincent, J., Bruchard, M., Remy-Martin, J. P., et al. (2010). Membrane-associated Hsp72 from tumor-derived exosomes mediates STAT3dependent immunosuppressive function of mouse and human myeloid-derived suppressor cells. The Journal of Clinical Investigation, 120, 457-471.

Chatterjee, S., Das, S., Chakraborty, P., Manna, A., Chatterjee, M., \& Choudhuri, S. K. (2013). Myeloid derived suppressor cells (MDSCs) can induce the generation of Th17 response from naive CD4 + T cells. Immunobiology, 218, 718-724.

Chen, S., Akbar, S. M., Miyake, T., Abe, M., Al-Mahtab, M., Furukawa, S., et al. (2015). Diminished immune response to vaccinations in obesity: Role of myeloid-derived suppressor and other myeloid cells. Obesity Research \& Clinical Practice, 9, 35-44.

Cheng, P., Corzo, C. A., Luetteke, N., Yu, B., Nagaraj, S., Bui, M. M., et al. (2008). Inhibition of dendritic cell differentiation and accumulation of myeloid-derived suppressor cells in cancer is regulated by $\mathrm{S} 100 \mathrm{~A} 9$ protein. The Journal of Experimental Medicine, 205, 2235-2249.

Cheng, P., Kumar, V., Liu, H., Youn, J. I., Fishman, M., Sherman, S., et al. (2014). Effects of notch signaling on regulation of myeloid cell differentiation in cancer. Cancer Researdh, $74,141-152$.

Chomoguz, O., Grmai, L., Sinha, P., Artemenko, K. A., Zubarev, R. A., \& OstrandRosenberg, S. (2011). Proteomic pathway analysis reveals inflammation increases myeloid-derived suppressor cell resistance to apoptosis. Molecular $\mathcal{E}$ Cellular Proteomics, 10, M110.002980. 
Chou, H. S., Hsieh, C. C., Charles, R., Wang, L., Wagner, T., Fung, J. J., et al. (2012). Myeloid-derived suppressor cells protect islet transplants by B7-H1 mediated enhancement of T regulatory cells. Transplantation, 93, 272-282.

Corzo, C. A., Condamine, T., Lu, L., Cotter, M. J., Youn, J. I., Cheng, P., et al. (2010). HIF-1 $\alpha$ regulates function and differentiation of myeloid-derived suppressor cells in the tumor microenvironment. The Journal of Expenimental Medicine, 207, 2439-2453.

Corzo, C. A., Cotter, M. J., Cheng, P., Cheng, F., Kusmartsev, S., Sotomayor, E., et al. (2009). Mechanism regulating reactive oxygen species in tumor-induced myeloidderived suppressor cells. Joumal of Immunology, 182, 5693-5701.

Cui, T. X., Kryczek, I., Zhao, L., Zhao, E., Kuick, R., Roh, M. H., et al. (2013). Myeloidderived suppressor cells enhance stemness of cancer cells by inducing microRNA101 and suppressing the corepressor CtBP2. Immunity, 39, 611-621.

Danelli, L., Frossi, B., Gri, G., Mion, F., Guarnotta, C., Bongiovanni, L., et al. (2015). Mast cells boost myeloid-derived suppressor cell activity and contribute to the development of tumor-favoring microenvironment. Cancer Immunology Reseanh, 3, 85-95.

Delano, M. J., Scumpia, P. O., Weinstein, J. S., Coco, D., Nagaraj, S., Kelly-Scumpia, K. M., et al. (2007). MyD88-dependent expansion of an immature GR-1(+)CD11b(+) population induces $\mathrm{T}$ cell suppression and $\mathrm{Th} 2$ polarization in sepsis. The Journal of Experimental Medicine, 204, 1463-1474.

Diaz-Montero, C. M., Salem, M. L., Nishimura, M. I., Garrett-Mayer, E., Cole, D. J., \& Montero, A. J. (2009). Increased circulating myeloid-derived suppressor cells correlate with clinical cancer stage, metastatic tumor burden, and doxorubicin-cyclophosphamide chemotherapy. Cancer Immunology, Immunotherapy, 58, 49-59.

Dolcetti, L., Peranzoni, E., Ugel, S., Marigo, I., Fernandez Gomez, A., Mesa, C., et al. (2010). Hierarchy of immunosuppressive strength among myeloid-derived suppressor cell subsets is determined by GM-CSF. European Joumal of Immunology, 40, $22-35$.

Dumitru, C. A., Moses, K., Trellakis, S., Lang, S., \& Brandau, S. (2012). Neutrophils and granulocytic myeloid-derived suppressor cells: Immunophenotyping, cell biology and clinical relevance in human oncology. Cancer Immunology, Immunotherapy, 61, 1155-1167.

Dunn, G. P., Bruce, A. T., Ikeda, H., Old, L. J., \& Schreiber, R. D. (2002). Cancer immunoediting: From immunosurveillance to tumor escape. Nature Immunology, 3, 991-998.

El Gazzar, M., \& McCall, C. E. (2012). MicroRNAs regulatory networks in myeloid lineage development and differentiation: Regulators of the regulators. Immunology and Cell Biology, 90, 587-593.

Elkabets, M., Ribeiro, V. S., Dinarello, C. A., Ostrand-Rosenberg, S., Di Santo, J. P., Apte, R. N., et al. (2010). IL-1beta regulates a novel myeloid-derived suppressor cell subset that impairs NK cell development and function. Eurpean Joumal of Immunology, 40, 3347-3357.

Enuslanov, E., Daurkin, I., Ortiz, J., Vieweg, J., \& Kusmartsev, S. (2010). Pivotal advance: Tumor-mediated induction of myeloid-derived suppressor cells and M2-polarized macrophages by altering intracellular $\mathrm{PGE}_{2}$ catabolism in myeloid cells. Journal of Leukocyte Biology, 88, 839-848.

Enuslanov, E., Neuberger, M., Daurkin, I., Perrin, G. Q., Algood, C., Dahm, P., et al. (2012). Circulating and tumor-infiltrating myeloid cell subsets in patients with bladder cancer. Intermational Journal of Cancer, 130, 1109-1119.

Espagnolle, N., Barron, P., Mandron, M., Blanc, I., Bonnin, J., Agnel, M., et al. (2014). Specific inhibition of the VEGFR-3 tyrosine kinase by SAR131675 reduces peripheral and tumor associated immunosuppressive myeloid cells. Cancers, 6, 472-490.

Ezernitchi, A. V., Vaknin, I., Cohen-Daniel, L., Levy, O., Manaster, E., Halabi, A., et al. (2006). TCR zeta down-regulation under chronic inflammation is mediated by myeloid 
suppressor cells differentially distributed between various lymphatic organs. Joumal of Immunology, 177, 4763-4772.

Farren, M. R., Carlson, L. M., \& Lee, K. P. (2010). Tumor-mediated inhibition of dendritic cell differentiation is mediated by down regulation of protein kinase $\mathrm{C}$ beta II expression. Immunologic Reseanh, 46, 165-176.

Fletcher, M., Ramirez, M. E., Sierra, R. A., Raber, P., Thevenot, P., Al-Khami, A. A., et al. (2015). L-Arginine depletion blunts antitumor T-cell responses by inducing myeloidderived suppressor cells. Cancer Research, 75, 275-283.

Forghani, P., Khorramizadeh, M. R., \& Waller, E. K. (2014). Silibinin inhibits accumulation of myeloid-derived suppressor cells and tumor growth of murine breast cancer. Cancer Medicine, 3, 215-224.

Fridlender, Z. G., Sun, J., Kim, S., Kapoor, V., Cheng, G., Ling, L., et al. (2009). Polarization of tumor-associated neutrophil phenotype by TGF-beta: "N1" versus "N2" TAN. Cancer Cell, 16, 183-194.

Fukumura, D., Kashiwagi, S., \& Jain, R. K. (2006). The role of nitric oxide in tumour progression. Nature Reviews. Cancer, 6, 521-534.

Gabitass, R. F., Annels, N. E., Stocken, D. D., Pandha, H. A., \& Middleton, G. W. (2011). Elevated myeloid-derived suppressor cells in pancreatic, esophageal and gastric cancer are an independent prognostic factor and are associated with significant elevation of the Th2 cytokine interleukin-13. Cancer Immunology, Immunotherapy, 60, 1419-1430.

Gabriele, L., Phung, J., Fukumoto, J., Segal, D., Wang, I. M., Giannakakou, P., et al. (1999). Regulation of apoptosis in myeloid cells by interferon consensus sequence-binding protein. The Journal of Experimental Medicine, 190, 411-421.

Gabrilovich, D. I., Bronte, V., Chen, S. H., Colombo, M. P., Ochoa, A., OstrandRosenberg, S., et al. (2007). The terminology issue for myeloid-derived suppressor cells. Cancer Researdh, 67, 425, author reply 426.

Gabrilovich, D., Ishida, T., Oyama, T., Ran, S., Kravtsov, V., Nadaf, S., et al. (1998). Vascular endothelial growth factor inhibits the development of dendritic cells and dramatically affects the differentiation of multiple hematopoietic lineages in vivo. Blood, 92, 4150-4166.

Gabrilovich, D. I., Velders, M. P., Sotomayor, E. M., \& Kast, W. M. (2001). Mechanism of immune dysfunction in cancer mediated by immature Gr-1+ myeloid cells. Journal of Immunology, 166, 5398-5406.

Garrity, T., Pandit, R., Wright, M. A., Benefield, J., Keni, S., \& Young, M. R. (1997). Increased presence of CD34 + cells in the peripheral blood of head and neck cancer patients and their differentiation into dendritic cells. Intemational Journal of Cancer, 73, 663-669.

Gehad, A. E., Lichtman, M. K., Schmults, C. D., Teague, J. E., Calarese, A. W., Jiang, Y., et al. (2012). Nitric oxide-producing myeloid-derived suppressor cells inhibit vascular E-selectin expression in human squamous cell carcinomas. The Journal of Investigative Dermatology, 132, 2642-2651.

Gleason, M. K., Ross, J. A., Warlick, E. D., Lund, T. C., Verneris, M. R., Wiemik, A., et al. (2014). CD16xCD33 bispecific killer cell engager (BiKE) activates NK cells against primary MDS and MDSC CD33 + targets. Blood, 123, 3016-3026.

Goñi, O., Alcaide, P., \& Fresno, M. (2002). Immunosuppression during acute Trypanosoma cruzi infection: Involvement of Ly6G $(\mathrm{Gr} 1(+)) \mathrm{CD} 11 \mathrm{~b}(+)$ immature myeloid suppressor cells. International Immunology, 14, 1125-1134.

Grizzle, W. E., Xu, X., Zhang, S., Stockard, C. R., Liu, C., Yu, S., et al. (2007). Age-related increase of tumor susceptibility is associated with myeloid-derived suppressor cell mediated suppression of $\mathrm{T}$ cell cytotoxicity in recombinant inbred BXD12 mice. Mechanisms of Ageing and Development, 128, 672-680. 
Haile, L. A., von Wasielewski, R., Gamrekelashvili, J., Krüger, C., Bachmann, O., Westendorf, A. M., et al. (2008). Myeloid-derived suppressor cells in inflammatory bowel disease: A new immunoregulatory pathway. Gastroenterology, 135, 871-881, 881.e1-5.

Hanson, E. M., Clements, V. K., Sinha, P., Ilkovitch, D., \& Ostrand-Rosenberg, S. (2009). Myeloid-derived suppressor cells down-regulate L-selectin expression on CD4+ and CD8 + T cells Journal of Immunology, 183, 937-944.

He, D., Li, H., Yusuf, N., Elmets, C. A., Li, J., Mountz, J. D., et al. (2010). IL-17 promotes tumor development through the induction of tumor promoting microenvironments at tumor sites and myeloid-derived suppressor cells. Journal of Immunology, 184, 2281-2288.

Highfill, S. L., Rodriguez, P. C., Zhou, Q., Goetz, C. A., Koehn, B. H., Veenstra, R., et al. (2010). Bone marrow myeloid-derived suppressor cells (MDSCs) inhibit graft-versushost disease (GVHD) via an arginase-1-dependent mechanism that is up-regulated by interleukin-13. Blood, 116, 5738-5747.

Hix, L. M., Karavitis, J., Khan, M. W., Shi, Y. H., Khazaie, K., \& Zhang, M. (2013). Tumor STAT1 transcription factor activity enhances breast tumor growth and immune suppression mediated by myeloid-derived suppressor cells. The Journal of Biological Chemistry, $288,11676-11688$.

Hoechst, B., Ormandy, L. A., Ballmaier, M., Lehner, F., Kruger, C., Manns, M. P., et al. (2008). A new population of myeloid-derived suppressor cells in hepatocellular carcinoma patients induces CD4(+)CD25(+)Foxp3(+) T cells. Gastroenterology, 135, 234-243.

Horlad, H., Fujiwara, Y., Takemura, K., Ohnishi, K., Ikeda, T., Tsukamoto, H., et al. (2013). Corosolic acid impairs tumor development and lung metastasis by inhibiting the immunosuppressive activity of myeloid-derived suppressor cells. Molecular Nutrition $\mathcal{E}$ Food Reseanch, 57, 1046-1054.

Hu, X., Bardhan, K., Paschall, A. V., Yang, D., Waller, J. L., Park, M. A., et al. (2013). Deregulation of apoptotic factors $\mathrm{Bcl}-\mathrm{xL}$ and $\mathrm{Bax}$ confers apoptotic resistance to myeloid-derived suppressor cells and contributes to their persistence in cancer. The Journal of Biological Chemistry, 288, 19103-19115.

Huang, B., Pan, P. Y., Li, Q., Sato, A. I., Levy, D. E., Bromberg, J., et al. (2006). Gr-1+CD115 + immature myeloid suppressor cells mediate the development of tumor-induced $\mathrm{T}$ regulatory cells and $\mathrm{T}$-cell anergy in tumor-bearing host. Cancer Researdh, 66, 1123-1131.

Huang, A., Zhang, B., Wang, B., Zhang, F., Fan, K. X., \& Guo, Y. J. (2013). Increased CD14(+)HLA-DR (-/low) myeloid-derived suppressor cells correlate with extrathoracic metastasis and poor response to chemotherapy in non-small cell lung cancer patients. Cancer Immunology, Immunotherapy, 62, 1439-1451.

Iclozan, C., Antonia, S., Chiappori, A., Chen, D. T., \& Gabrilovich, D. (2013). Therapeutic regulation of myeloid-derived suppressor cells and immune response to cancer vaccine in patients with extensive stage small cell lung cancer. Cancer Immunology, Immunotherapy, 62, 909-918.

Ilkovitch, D., \& Lopez, D. M. (2009). The liver is a site for tumor-induced myeloid-derived suppressor cell accumulation and immunosuppression. Cancer Reseanch, 69, 5514-5521.

Ioannou, M., Alissafi, T., Lazaridis, I., Deraos, G., Matsoukas, J., Gravanis, A., et al. (2012). Crucial role of granulocytic myeloid-derived suppressor cells in the regulation of central nervous system autoimmune disease. Joumal of Immunology, 188, 1136-1146.

Jablonska, J., Leschner, S., Westphal, K., Lienenklaus, S., \& Weiss, S. (2010). Neutrophils responsive to endogenous IFN-beta regulate tumor angiogenesis and growth in a mouse tumor model. The Joumal of Clinical Investigation, 120, 1151-1164.

Karin, M. (2006). Nuclear factor-kappaB in cancer development and progression. Nature, $441,431-436$. 
Kim, K., Skora, A. D., Li, Z., Liu, Q., Tam, A. J., Blosser, R. L., et al. (2014). Eradication of metastatic mouse cancers resistant to immune checkpoint blockade by suppression of myeloid-derived cells. Proceedings of the National Acadeny of Sciences of the United States of America, 111, 11774-11779.

Kimura, T., McKolanis, J. R., Dzubinski, L. A., Islam, K., Potter, D. M., Salazar, A. M., et al. (2013). MUC1 vaccine for individuals with advanced adenoma of the colon: A cancer immunoprevention feasibility study. Cancer Prevention Reseanch (Philadelphia, PA), 6, $18-26$.

Köstlin, N., Kugel, H., Spring, B., Leiber, A., Marmé, A., Henes, M., et al. (2014). Granulocytic myeloid derived suppressor cells expand in human pregnancy and modulate T-cell responses. European Journal of Immunology, 44, 2582-2591.

Kowanetz, M., Wu, X., Lee, J., Tan, M., Hagenbeek, T., Qu, X., et al. (2010). Granulocyte-colony stimulating factor promotes lung metastasis through mobilization of Ly6G+Ly6C + granulocytes. Proceedings of the National Academy of Sciences of the United States of America, 107, 21248-21255.

Kujawski, M., Kortylewski, M., Lee, H., Hermann, A., Kay, H., \& Yu, H. (2008). Stat3 mediates myeloid cell-dependent tumor angiogenesis in mice. The Joumal of Clinical Investigation, 118, 3367-3377.

Kusmartsev, S., Eruslanov, E., Kübler, H., Tseng, T., Sakai, Y., Su, Z., et al. (2008). Oxidative stress regulates expression of VEGFR1 in myeloid cells: Link to tumorinduced immune suppression in renal cell carcinoma. Journal of Immunology, 181, 346-353.

Kusmartsev, S., \& Gabrilovich, D. I. (2005). STAT1 signaling regulates tumor-associated macrophage-mediated T cell deletion. Journal of Immunology, 174, 4880-4891.

Levring, T. B., Hansen, A. K., Nielsen, B. L., Kongsbak, M., von Essen, M. R., Woetmann, A., et al. (2012). Activated human CD4+ T cells express transporters for both cysteine and cystine. Saientific Reports, 2, 266.

Li, W., Wu, K., Zhao, E, Shi, L., Li, R., Zhang, P., et al. (2013). HMGB1 recruits myeloid derived suppressor cells to promote peritoneal dissemination of colon cancer after resection. Biochemical and Biophysical Research Communications, 436, 156-161.

Li, L., Zhang, J., Diao, W., Wang, D., Wei, Y., Zhang, C. Y., et al. (2014). MicroRNA-155 and MicroRNA-21 promote the expansion of functional myeloid-derived suppressor cells. Journal of Immunology, 192, 1034-1043.

Lieschke, G. J., Grail, D., Hodgson, G., Metcalf, D., Stanley, E., Cheers, C., et al. (1994). Mice lacking granul ocyte colony-stimulating factor have chronic neutropenia, granulocyte and macrophage progenitor cell deficiency, and impaired neutrophil mobilization. Blood, 84, 1737-1746.

Lin, Y., Gustafson, M. P., Bulur, P. A., Gastineau, D. A., Witzig, T. E., \& Dietz, A. B. (2011). Immunosuppressive CD14+HLA-DR(low)/- monocytes in B-cell nonHodgkin lymphoma. Blood, 117, 872-881.

Liu, Y., Lai, L., Chen, Q., Song, Y., Xu, S., Ma, F., et al. (2012). MicroRNA-494 is required for the accumulation and functions of tumor-expanded myeloid-derived suppressor cells via targeting of PTEN. Journal of Immunology, 188, 5500-5510.

Liu, C., Yu, S., Kappes, J., Wang, J., Grizzle, W. E., Zinn, K. R., et al. (2007). Expansion of spleen myeloid suppressor cells represses NK cell cytotoxicity in tumor-bearing host. Blood, 109, 4336-4342.

Liu, Q., Zhang, M., Jiang, X., Zhang, Z., Dai, L., Min, S., et al. (2011). miR-223 suppresses differentiation of tumor-induced $\mathrm{CD} 11 \mathrm{~b}^{+} \mathrm{Gr} 1^{+}$myeloid-derived suppressor cells from bone marrow cells. International Joumal of Cancer, 129, 2662-2673.

Lu, T., \& Gabrilovich, D. I. (2012). Molecular pathways: Tumor-infiltrating myeloid cells and reactive oxygen species in regulation of tumor microenvironment. Clinical Cancer Research, 18, 4877-4882. 
Lu, T., Ramakrishnan, R., Altiok, S., Youn, J. I., Cheng, P., Celis, E., et al. (2011). Tumorinfiltrating myeloid cells induce tumor cell resistance to cytotoxic $\mathrm{T}$ cells in mice. The Journal of Clinical Investigation, 121, 4015-4029.

MacDonald, K. P., Rowe, V., Clouston, A. D., Welply, J. K., Kuns, R. D., Ferrara, J. L., et al. (2005). Cytokine expanded myeloid precursors function as regulatory antigenpresenting cells and promote tolerance through IL-10-producing regulatory T cells. Journal of Immunology, 174, 1841-1850.

Makarenkova, V. P., Bansal, V., Matta, B. M., Perez, L. A., \& Ochoa, J. B. (2006). CD11b+/ Gr-1 + myeloid suppressor cells cause $\mathrm{T}$ cell dysfunction after traumatic stress. Journal of Immunology, 176, 2085-2094.

Mao, Y., Sarhan, D., Steven, A., Seliger, B., Kiessling, R., \& Lundqvist, A. (2014). Inhibition of tumor-derived prostaglandin-e2 blocks the induction of myeloid-derived suppressor cells and recovers natural killer cell activity. Clinical Cancer Researdh, 20, 4096-4106.

Marigo, I., Bosio, E., Solito, S., Mesa, C., Fernandez, A., Dolcetti, L., et al. (2010). Tumorinduced tolerance and immune suppression depend on the $\mathrm{C} / \mathrm{EBPbeta}$ transcription factor. Immunity, 32, 790-802.

Markiewski, M. M., DeAngelis, R. A., Benencia, F., Ricklin-Lichtsteiner, S. K., Koutoulaki, A., Gerard, C., et al. (2008). Modulation of the antitumor immune response by complement. Nature Immunology, 9, 1225-1235.

Martin, R. K., Saleem, S. J., Folgosa, L., Zellner, H. B., Damle, S. R., Nguyen, G. K., et al. (2014). Mast cell histamine promotes the immunoregulatory activity of myeloid-derived suppressor cells. Joumal of Leukocyte Biology, 96, 151-159.

Mazzoni, A., Bronte, V., Visintin, A., Spizzer, J. H., Apolloni, E., Serafini, P., et al. (2002). Myeloid suppressor lines inhibit T cell responses by an NO-dependent mechanism. Journal of Immunology, 168, 689-695.

Melani, C., Chiodoni, C., Forni, G., \& Colombo, M. P. (2003). Myeloid cell expansion elicited by the progression of spontaneous mammary carcinomas in c-erbB-2 transgenic BALB/c mice suppresses immune reactivity. Blood, 102, 2138-2145.

Melani, C., Sangaletti, S., Barazzetta, F. M., Werb, Z., \& Colombo, M. P. (2007). Aminobiphosphonate-mediated MMP-9 inhibition breaks the tumor-bone marrow axis responsible for myeloid-derived suppressor cell expansion and macrophage infiltration in tumor stroma. Cancer Reseanh, 67, 11438-11446.

Mikyskova, R., Indrova, M., Vlkova, V., Bieblova, J., Simova, J., Parackova, Z., et al. (2014). DNA demethylating agent 5-azacytidine inhibits myeloid-derived suppressor cells induced by tumor growth and cyclophosphamide treatment. Journal of Leukocyte Biology, 95, 743-753.

Molon, B., Ugel, S., Del Pozzo, F., Soldani, C., Zilio, S., Avella, D., et al. (2011). Chemokine nitration prevents intratumoral infiltration of antigen-specific $\mathrm{T}$ cells. The Journal of Experimental Mediane, 208, 1949-1962.

Montero, A. J., Diaz-Montero, C. M., Kyriakopoulos, C. E., Bronte, V., \& Mandruzzato, S. (2012). Myeloid-derived suppressor cells in cancer patients a clinical perspective. Journal of Immunothenapy, 35, 107-115.

Morales, J. K., Kmieciak, M., Knutson, K. L., Bear, H. D., \& Manjili, M. H. (2010). GM-CSF is one of the main breast tumor-derived soluble factors involved in the differentiation of CD11b-Gr1- bone marrow progenitor cells into myeloid-derived suppressor cells. Breast Cancer Researd and Treatment, 123, 39-49.

Mostafa, M. H., Sheweita, S. A., \& O'Connor, P. J. (1999). Relationship between schistosomiasis and bladder cancer. Clinical Microbiology Revieus, 12, 97-111.

Movahedi, K., Guilliams, M., Van den Bossche, J., Van den Bergh, R., Gysemans, C., Beschin, A., et al. (2008). Identification of discrete tumor-induced myeloid-derived suppressor cell subpopulations with distinct $\mathrm{T}$ cell-suppressive activity. Blood, 111, 4233-4244. 
Mucha, J., Majchrzak, K., Taciak, B., Hellmen, E., \& Krol, M. (2014). MDSCs mediate angiogenesis and predispose canine mammary tumor cells for metastasis via IL-28/IL28RA (IFN-lambda) signaling. PLoS One, 9, e103249.

Mundy-Bosse, B. L., Thornton, L. M., Yang, H. C., Andersen, B. L., \& Carson, W. E. (2011). Psychological stress is associated with altered levels of myeloid-derived suppressor cells in breast cancer patients. Cellular Immunology, 270, 80-87.

Munn, D. H., Sharma, M. D., Baban, B., Harding, H. P., Zhang, Y., Ron, D., et al. (2005). GCN2 kinase in T cells mediates proliferative arrest and anergy induction in response to indoleamine 2,3-dioxygenase. Immunity, 22, 633-642.

Nefedova, Y., Cheng, P., Gilkes, D., Blaskovich, M., Beg, A. A., Sebti, S. M., et al. (2005). Activation of dendritic cells via inhibition of Jak2/STAT3 signaling. Joumal of Immunology, 175, 4338-4346.

Nefedova, Y., Huang, M., Kusmartsev, S., Bhattacharya, R., Cheng, P., Salup, R., et al. (2004). Hyperactivation of STAT3 is involved in abnormal differentiation of dendritic cells in cancer. Joumal of Immunology, 172, 464-474.

Noman, M. Z., Desantis, G., Janji, B., Hasmim, M., Karray, S., Dessen, P., et al. (2014). PD-L1 is a novel direct target of HIF-1alpha, and its blockade under hypoxia enhanced MDSC-mediated $\mathrm{T}$ cell activation. The Journal of Experimental Medicine, $211,781-790$.

Obermajer, N., Muthuswamy, R., Lesnock, J., Edwards, R. P., \& Kalinski, P. (2011). Positive feedback between PGE2 and COX2 redirects the differentiation of human dendritic cells toward stable myeloid-derived suppressor cells. Blood, 118, 5498-5505.

Okazaki, T., Ebihara, S., Asada, M., Kanda, A., Sasaki, H., \& Yamaya, M. (2006). Granulocyte colony-stimulating factor promotes tumor angiogenesis via increasing circulating endothelial progenitor cells and Gr1+CD11b + cells in cancer animal models. International Immunology, 18, 1-9.

Ortiz, M. L., Kumar, V., Martner, A., Mony, S., Donthireddy, L., Condamine, T., et al. (2015). Immature myeloid cells directly contribute to skin tumor development by recruiting IL-17-producing CD4+ T cells. The Journal of Experimental Mediane, 212, 351-367.

Ostrand-Rosenberg, S., \& Sinha, P. (2009). Myeloid-derived suppressor cells: Linking inflammation and cancer. Joumal of Immunology, 182, 4499-4506.

OuYang, L. Y., Wu, X. J., Ye, S. B., Zhang, R. X., Li, Z. L., Liao, W., et al. (2015). Tumorinduced myeloid-derived suppressor cells promote tumor progression through oxidative metabolism in human colorectal cancer. Journal of Translational Mediane, 13, 47.

Pan, P. Y., Ma, G., Weber, K. J., Ozao-Choy, J., Wang, G., Yin, B., et al. (2010). Immune stimulatory receptor CD40 is required for $\mathrm{T}$-cell suppression and $\mathrm{T}$ regulatory cell activation mediated by myeloid-derived suppressor cells in cancer. Cancer Reseanch, 70, 99-108.

Panka, D. J., Liu, Q., Geissler, A. K., \& Mier, J. W. (2013). Effects of HDM2 antagonism on sunitinib resistance, p53 activation, SDF-1 induction, and tumor infiltration by CD11b+/Gr-1 + myeloid derived suppressor cells. Molecular Cancer, 12, 17.

Parker, K., Sinha, P., Hom, L., Clements, V., \& Ostrand-Rosenberg, S. (2014). HMGB1 enhances immune suppression by facilitating the differentiation and suppressive activity of myeloid-derived suppressor cells. Cancer Research, 74, 5723-5733.

Porembka, M. R., Mitchem, J. B., Belt, B. A., Hsieh, C. S., Lee, H. M., Hemdon, J., et al. (2012). Pancreatic adenocarcinoma induces bone marrow mobilization of myeloidderived suppressor cells which promote primary tumor growth. Cancer Immunology, Immunotherapy, 61, 1373-1385.

Punturieri, A., Szabo, E., Croxton, T. L., Shapiro, S. D., \& Dubinett, S. M. (2009). Lung cancer and chronic obstructive pulmonary disease: Needs and opportunities for integrated research. Journal of the National Cancer Institute, 101, 554-559. 
Raber, P. L., Thevenot, P., Sierra, R., Wyczechowska, D., Halle, D., Ramirez, M. E., et al. (2014). Subpopulations of myeloid-derived suppressor cells impair T cell responses through independent nitric oxide-related pathways. Intemational Joumal of Cancer, 134, 2853-2864.

Rodriguez, P. C., Emstoff, M. S., Hernandez, C., Atkins, M., Zabaleta, J., Sierra, R., et al. (2009). Arginase I-producing myeloid-derived suppressor cells in renal cell carcinoma are a subpopulation of activated granulocytes. Cancer Reseanh, 69, 1553-1560.

Rodriguez, P. C., Hernandez, C. P., Morrow, K., Sierra, R., Zabaleta, J., Wyczechowska, D. D., et al. (2010). L-Arginine deprivation regulates cyclin D3 mRNA stability in human $\mathrm{T}$ cells by controlling HuR expression. Joumal of Immunology, 185, 5198-5204.

Rodriguez, P. C., Hemandez, C. P., Quiceno, D., Dubinett, S. M., Zabaleta, J., Ochoa, J. B., et al. (2005). Arginase I in myeloid suppressor cells is induced by COX-2 in lung carcinoma. The Joumal of Experimental Mediane, 202, 931-939.

Rodriguez, P. C., Quiceno, D. G., Zabaleta, J., Ortiz, B., Zea, A. H., Piazuelo, M. B., et al. (2004). Arginase I production in the tumor microenvironment by mature myeloid cells inhibits $\mathrm{T}$-cell receptor expression and antigen-specific $\mathrm{T}$-cell responses. Cancer Research, $64,5839-5849$.

Rodriguez, P. C., Zea, A. H., Culotta, K. S., Zabaleta, J., Ochoa, J. B., \& Ochoa, A. C. (2002). Regulation of T cell receptor CD3zeta chain expression by L-arginine. The Journal of Biological Chemistry, 277, 21123-21129.

Sakamaki, I., Kwak, L. W., Cha, S. C., Yi, Q., Lerman, B., Chen, J., et al. (2014). Lenalidomide enhances the protective effect of a therapeutic vaccine and reverses immune suppression in mice bearing established lymphomas. Leukemia, 28, 329-337.

Saleem, S. J., Martin, R. K., Morales, J. K., Sturgill, J. L., Gibb, D. R., Graham, L., et al. (2012). Cutting edge: Mast cells critically augment myeloid-derived suppressor cell activity. Journal of Immunology, 189, 511-515.

Santhanam, L., Lim, H. K., Miriel, V., Brown, T., Patel, M., Balanson, S., et al. (2007). Inducible NO synthase dependent S-nitrosylation and activation of arginase1 contribute to age-related endothelial dysfunction. Cinculation Research, 101, 692-702.

Santilli, G., Piotrowska, I., Cantilena, S., Chayka, O., D'Alicarnasso, M., Morgenstern, D. A., et al. (2013). Polyphenon [corrected] E enhances the antitumor immune response in neuroblastoma by inactivating myeloid suppressor cells. Clinical Cancer Researdh, 19, 1116-1125.

Schilling, B., Sucker, A., Griewank, K., Zhao, F., Weide, B., Gorgens, A., et al. (2013). Vemurafenib reverses immunosuppression by myeloid derived suppressor cells. Intemational Journal of Cancer, 133, 1653-1663.

Schmielau, J., \& Finn, O. J. (2001). Activated granulocytes and granulocyte-derived hydrogen peroxide are the underlying mechanism of suppression of $\mathrm{t}$-cell function in advanced cancer patients. Cancer Research, 61, 4756-4760.

Serafini, P. (2013). Myeloid derived suppressor cells in physiological and pathological conditions: The good, the bad, and the ugly. Immunologic Research, 57, 172-184.

Serafini, P., Carbley, R., Noonan, K. A., Tan, G., Bronte, V., \& Borrello, I. (2004). Highdose granulocyte-macrophage colony-stimulating factor-producing vaccines impair the immune response through the recruitment of myeloid suppressor cells. Cancer Research, $64,6337-6343$.

Serafini, P., Mgebroff, S., Noonan, K., \& Borrello, I. (2008). Myeloid-derived suppressor cells promote cross-tolerance in B-cell lymphoma by expanding regulatory $\mathrm{T}$ cells. Cancer Researdh, 68, 5439-5449.

Shacter, E., \& Weitzman, S. A. (2002). Chronic inflammation and cancer. Oncology (Williston Park), 16, 217-226, 229; discussion 230-232. 
Shen, P., Wang, A., He, M., Wang, Q., \& Zheng, S. (2014). Increased circulating Lin(-/low) CD33(+) HLA-DR(-) myeloid-derived suppressor cells in hepatocellular carcinoma patients. Hepatology Researdh, 44, 639-650.

Sica, A., \& Mantovani, A. (2012). Macrophage plasticity and polarization: In vivo veritas. The Journal of Clinical Investigation, 122, 787-795.

Sinha, P., Chornoguz, O., Clements, V. K., Artemenko, K. A., Zubarev, R. A., \& OstrandRosenberg, S. (2011). Myeloid-derived suppressor cells express the death receptor Fas and apoptose in response to T cell-expressed FasL. Blood, 117, 5381-5390.

Sinha, P., Clements, V. K., Bunt, S. K., Albelda, S. M., \& Ostrand-Rosenberg, S. (2007). Cross-talk between myeloid-derived suppressor cells and macrophages subverts tumor immunity toward a type 2 response. Journal of Immunology, 179, 977-983.

Sinha, P., Clements, V. K., Fulton, A. M., \& Ostrand-Rosenberg, S. (2007). Prostaglandin E2 promotes tumor progression by inducing myeloid-derived suppressor cells. Cancer Researd, 67, 4507-4513.

Sinha, P., Clements, V. K., \& Ostrand-Rosenberg, S. (2005a). Interleukin-13-regulated M2 macrophages in combination with myeloid suppressor cells block immune surveillance against metastasis. Cancer Reseanh, 65, 11743-11751.

Sinha, P., Clements, V. K., \& Ostrand-Rosenberg, S. (2005b). Reduction of myeloidderived suppressor cells and induction of M1 macrophages facilitate the rejection of established metastatic disease. Journal of Immunology, 174, 636-645.

Sinha, P., Okoro, C., Foell, D., Freeze, H. H., Ostrand-Rosenberg, S., \& Srikrishna, G. (2008). Proinflammatory S100 proteins regulate the accumulation of myeloid-derived suppressor cells. Journal of Immunology, 181, 4666-4675.

Sinha, P., \& Ostrand-Rosenberg, S. (2013). Myeloid-derived suppressor cell function is reduced by Withaferin $A$, a potent and abundant component of Withania somnifera root extract. Cancer Immunology, Immunothenapy, 62, 1663-1673.

Sinha, P., Parker, K. H., Hom, L., \& Ostrand-Rosenberg, S. (2012). Tumor-induced myeloid-derived suppressor cell function is independent of IFN- $\gamma$ and IL-4R $\alpha$. European Journal of Immunology, 42, 2052-2059.

Sionov, R. V., Fridlender, Z G., \& Granot, Z. (2014). The multifaceted roles neutrophils play in the tumor microenvironment. Cancer Microenvionment.

Smith, C., Chang, M. Y., Parker, K. H., Beury, D. W., DuHadaway, J. B., Flick, H. E., et al. (2012). IDO is a nodal pathogenic driver of lung cancer and metastasis development. Cancer Discovery, 2, 722-735.

Song, X., Krelin, Y., Dvorkin, T., Bjorkdahl, O., Segal, S., Dinarello, C. A., et al. (2005). CD11b + / Gr-1 + immature myeloid cells mediate suppression of $\mathrm{T}$ cells in mice bearing tumors of IL-1beta-secreting cells. Journal of Immunology, 175, 8200-8208.

Song, C., Yuan, Y., Wang, X. M., Li, D., Zhang, G. M., Huang, B., et al. (2014). Passive transfer of tumour-derived MDSCs inhibits asthma-related airway inflammation. Scandinavian Journal of Immunology, 79, 98-104.

Srivastava, M. K., Bosch, J. J., Thompson, J. A., Ksander, B. R., Edelman, M. J., \& OstrandRosenberg, S. (2008). Lung cancer patients' CD4(+) T cells are activated in vitro by MHC II cell-based vaccines despite the presence of myeloid-derived suppressor cells. Cancer Immunology, Immunotherapy, 57, 1493-1504.

Srivastava, M. K., Sinha, P., Clements, V. K., Rodriguez, P., \& Ostrand-Rosenberg, S. (2010). Myeloid-derived suppressor cells inhibit T-cell activation by depleting cystine and cysteine. Cancer Researdh, 70, 68-77.

Stewart, T. J., Liewehr, D. J., Steinberg, S. M., Greeneltch, K. M., \& Abrams, S. I. (2009). Modulating the expression of IFN regulatory factor 8 alters the protumorigenic behavior of CD11b+Gr-1 + myeloid cells. Joumal of Immunology, 183, 117-128.

Strober, S. (1984). Natural suppressor (NS) cells, neonatal tolerance, and total lymphoid irradiation: Exploring obscure relationships. Anmual Review of Immunology, 2, 219-237. 
Terabe, M., Matsui, S., Park, J. M., Mamura, M., Noben-Trauth, N., Donaldson, D. D., et al. (2003). Transforming growth factor-beta production and myeloid cells are an effector mechanism through which CD1d-restricted $\mathrm{T}$ cells block cytotoxic T lymphocyte-mediated tumor immunosurveillance: Abrogation prevents tumor recurrence. The Journal of Experimental Medicine, 198, 1741-1752.

Thevenot, P. T., Sierra, R. A., Raber, P. L., Al-Khami, A. A., Trillo-Tinoco, J., Zarreii, P., et al. (2014). The stress-response sensor chop regulates the function and accumulation of myeloid-derived suppressor cells in tumors. Immunity, 41, 389-401.

Tu, S., Bhagat, G., Cui, G., Takaishi, S., Kurt-Jones, E. A., Rickman, B., et al. (2008). Overexpression of interleukin-1beta induces gastric inflammation and cancer and mobilizes myeloid-derived suppressor cells in mice. Cancer Cell, 14, 408-419.

Vakkila, J., \& Lotze, M. T. (2004). Inflammation and necrosis promote tumour growth. Nature Reviews. Immunology, 4, 641-648.

Venereau, E., Casalgrandi, M., Schiraldi, M., Antoine, D. J., Cattaneo, A., De Marchis, F., et al. (2012). Mutually exclusive redox forms of HMGB1 promote cell recruitment or proinflammatory cytokine release. The Joumal of Experimental Mediane, 209, 1519-1528.

Verschoor, C. P., Johnstone, J., Millar, J., Dorrington, M. G., Habibagahi, M., Lelic, A., et al. (2013). Blood CD33(+)HLA-DR(-) myeloid-derived suppressor cells are increased with age and a history of cancer. Joumal of Leukocyte Biology, 93, 633-637.

Voisin, M. B., Buzoni-Gatel, D., Bout, D., \& Velge-Roussel, F. (2004). Both expansion of regulatory GR1 + CD11b+ myeloid cells and anergy of T lymphocytes participate in hyporesponsiveness of the lung-associated immune system during acute toxoplasmosis. Infection and Immunity, 72, 5487-5492.

Vuk-Pavlović, S., Bulur, P. A., Lin, Y., Qin, R., Szumlanski, C. L., Zhao, X., et al. (2010). Immunosuppressive CD14+HLA-DRlow/-monocytes in prostate cancer. Prostate, 70, 443-455.

Waight, J. D., Netherby, C., Hensen, M. L., Miller, A., Hu, Q., Liu, S., et al. (2013). Myeloid-derived suppressor cell development is regulated by a STAT/IRF-8 axis. The Journal of Clinical Investigation, 123, 4464-4478.

Wang, L., Chang, E. W., Wong, S. C., Ong, S. M., Chong, D. Q., \& Ling, K. L. (2013). Increased myeloid-derived suppressor cells in gastric cancer correlate with cancer stage and plasma S100A8/A9 proinflammatory proteins. Joumal of Immunology, 190, 794-804.

Wang, R., \& Green, D. R. (2012). Metabolic checkpoints in activated T cells. Nature Immunology, 13, 907-915.

Wang, L., Yi, T., Kortylewski, M., Pardoll, D. M., Zeng, D., \& Yu, H. (2009). IL-17 can promote tumor growth through an IL-6-Stat3 signaling pathway. The Journal of Experimental Medicine, 206, 1457-1464.

Weed, D. T., Vella, J. L., Reis, I. M., De la Fuente, A. C., Gomez, C., Sargi, Z., et al. (2015). Tadalafil reduces myeloid-derived suppressor cells and regulatory $T$ cells and promotes tumor immunity in patients with head and neck squamous cell carcinoma. Clinical Cancer Research, 21, 39-48.

Wesolowski, R., Markowitz, J., \& Carson, W. E., 3rd. (2013). Myeloid derived suppressor cells-A new therapeutic target in the treatment of cancer. Journal for Immunotherapy of Cancer, $1,10$.

Wu, S., Rhee, K. J., Albesiano, E, Rabizadeh, S., Wu, X., Yen, H. R., et al. (2009). A human colonic commensal promotes colon tumorigenesis via activation of T helper type $17 \mathrm{~T}$ cell responses. Nature Mediane, 15, 1016-1022.

Wu, J., Zhang, R., Tang, N., Gong, Z., Zhou, J., Chen, Y., et al. (2015). Dopamine inhibits the function of Gr-1+CD115+ myeloid-derived suppressor cells through D1-like receptors and enhances anti-tumor immunity. Journal of Leukocyte Biology, 97, 191-200. 
Xia, S., Sha, H., Yang, L., Ji, Y., Ostrand-Rosenberg, S., \& Qi, L. (2011). Gr-1 + CD11b+ myeloid-derived suppressor cells suppress inflammation and promote insulin sensitivity in obesity. The Journal of Biological Chemistry, 286, 23591-23599.

Xin, H., Zhang, C., Herrmann, A., Du, Y., Figlin, R., \& Yu, H. (2009). Sunitinib inhibition of Stat 3 induces renal cell carcinoma tumor cell apoptosis and reduces immunosuppressive cells. Cancer Research, 69, 2506-2513.

Yazawa, T., Shibata, M., Gonda, K., Machida, T., Suzuki, S., Kenjo, A., et al. (2013). Increased IL-17 production correlates with immunosuppression involving myeloidderived suppressor cells and nutritional impairment in patients with various gastrointestinal cancers. Molecular and Clinical Oncology, 1, 675-679.

Ye, J., Livergood, R. S., \& Peng, G. (2013). The role and regulation of human Th17 cells in tumor immunity. The American Joumal of Pathology, 182, 10-20.

Yin, Y., Huang, X., Lynn, K. D., \& Thorpe, P. E. (2013). Phosphatidylserine-targeting antibody induces M1 macrophage polarization and promotes myeloid-derived suppressor cell differentiation. Cancer Immunology Reseanh, 1, 256-268.

Yin, B., Ma, G., Yen, C. Y., Zhou, Z., Wang, G. X., Divino, C. M., et al. (2010). Myeloidderived suppressor cells prevent type 1 diabetes in murine models. Journal of Immunology, $185,5828-5834$.

Youn, J. I., Collazo, M., Shalova, I. N., Biswas, S. K., \& Gabrilovich, D. I. (2012). Characterization of the nature of granulocytic myeloid-derived suppressor cells in tumorbearing mice. Journal of Leukocyte Biology, 91, 167-181.

Youn, J. I., Nagaraj, S., Collazo, M., \& Gabrilovich, D. I. (2008). Subsets of myeloid-derived suppressor cells in tumor-bearing mice. Joumal of Immunology, 181, 5791-5802.

Young, M. R., Petruzzelli, G. J., Kolesiak, K., Achille, N., Lathers, D. M., \& Gabrilovich, D. I. (2001). Human squamous cell carcinomas of the head and neck chemoattract immune suppressive CD34(+) progenitor cells. Human Immunology, 62, $332-341$.

Yu, J., Du, W., Yan, F., Wang, Y., Li, H., Cao, S., et al. (2013). Myeloid-derived suppressor cells suppress antitumor immune responses through IDO expression and correlate with lymph node metastasis in patients with breast cancer. Journal of Immunology, 190, 3783-3797.

Yuan, H., Cai, P., Li, Q., Wang, W., Sun, Y., Xu, Q., et al. (2014). Axitinib augments antitumor activity in renal cell carcinoma via STAT3-dependent reversal of myeloid-derived suppressor cell accumulation. Biomediane $\&$ Pharmacothenapy, 68, 751-756.

Zea, A. H., Rodriguez, P. C., Atkins, M. B., Hernandez, C., Signoretti, S., Zabaleta, J., et al. (2005). Arginase-producing myeloid suppressor cells in renal cell carcinoma patients: A mechanism of tumor evasion. Cancer Reseanch, 65, 3044-3048.

Zea, A. H., Rodriguez, P. C., Culotta, K. S., Hernandez, C. P., DeSalvo, J., Ochoa, J. B., et al. (2004). L-Arginine modulates CD3zeta expression and T cell function in activated human T lymphocytes. Cellular Immunology, 232, 21-31.

Zhang, M., Liu, Q., Mi, S., Liang, X., Zhang, Z, Su, X., et al. (2011). Both miR-17-5p and miR-20a alleviate suppressive potential of myeloid-derived suppressor cells by modulating STAT3 expression. Journal of Immunology, 186, 4716-4724.

Zhang, Y. L., Luan, B., Wang, X. F., Qiao, J. Y., Song, L., Lei, R. R., et al. (2013). Peripheral blood MDSCs, IL-10 and IL-12 in children with asthma and their importance in asthma development. PLoS One, 8, e63775.

Zhang, B., Wang, Z., Wu, L., Zhang, M., Li, W., Ding, J., et al. (2013). Circulating and tumor-infiltrating myeloid-derived suppressor cells in patients with colorectal carcinoma. PLoS One, 8, e57114.

Zhu, B., Bando, Y., Xiao, S., Yang, K., Anderson, A. C., Kuchroo, V. K., et al. (2007). $\mathrm{CD} 1 \mathrm{~b}+\mathrm{Ly}-6 \mathrm{C}(\mathrm{hi})$ suppressive monocytes in experimental autoimmune encephalomyelitis. Journal of Immunology, 179, 5228-5237. 
Zitvogel, L., Apetoh, L., Ghiringhelli, F., \& Kroemer, G. (2008). Immunological aspects of cancer chemotherapy. Nature Revieus. Immunology, 8, 59-73.

Zong, W. X., \& Thompson, C. B. (2006). Necrotic death as a cell fate. Genes $\&$ Development, $20,1-15$.

Zoso, A., Mazza, E. M., Bicciato, S., Mandruzzato, S., Bronte, V., Serafini, P., et al. (2014). Human fibrocytic myeloid-derived suppressor cells express IDO and promote tolerance via Treg-cell expansion. Eunopean Joumal of Immunology, 44, 3307-3319. 
Appendix 4: Cross-talk between myeloid-derived suppressor cells (MDSC), macrophages, and dendritic cells enhances tumor-induced immune suppression 
Review

\title{
Cross-talk between myeloid-derived suppressor cells (MDSC), macrophages, and dendritic cells enhances tumor-induced immune suppression
}

\author{
Suzanne Ostrand-Rosenberg *, Pratima Sinha, Daniel W. Beury, Virginia K. Clements \\ Universtly of Maryiund Bantimore County, Dept. Blologicul Sciences, United States
}

\section{A R TICLE I N F O}

\section{Keywords:}

Tumor immunity

Immune escape

Tumar microenvironment

Tumar-associated macrophages

\begin{abstract}
A B S T R A C T
The tumor microenvironment is a complex milieu of tumor and host cells. Host cells can include tumorreactive T cells capable of killing tumor cells. However, more frequently the tumor and host components interact to generate a highly immune suppressive environment that frustrates T cell cytotoxicity and promotes tumor progression through a variety of immune and non-immune mechanisms. Myeloid-derived suppressor cells (MDSC) are a major host component contributing to the immune suppressive environment. In addition to their inherent immune suppressive function, MDSC amplify the immune suppressive activity of macrophages and dendritic cells via cross-talk. This article will review the cell-cell interactions used by MDSC to inhibit anti-tumor immunity and promote progression, and the role of inflammation in promoting cross-talk between MDSC and other cells in the tumor microenvironment.
\end{abstract}

Q 2012 Published by Elsevier Ltd.
The past decade has seen a large expansion in the number of experimental cancer immunotherapies being tested in clinical trials. Despite isolated responses in some patients, most of these trials have shown minimal impact on the clinical status of the majority of patients. The exceptions have been trials focused on reducing immune suppressive mechanisms that are present in most cancer patients, and include treatment with the mAbs ipilimumab and MDX-1106 which inhibit Cytotoxic T Lymphocyte Activation4 (CTLA-4) [1,2] and Programmed Death-1 (PD-1) [3] inhibitory molecules on T cells, respectively. In conjunction with extensive animal data showing that immune suppression is wide-spread in cancer patients, these clinical trials indicate that successful cancer immunotherapy will necessitate decreasing/eliminating immune suppressive mechanisms, In addition to suppression via molecules such as CTLA-4 and PD-1, myeloid lineage cells constitute a network of immune suppressive cells that are present in most cancer patients and which profoundly inhibit the generation of anti-tumor immunity. This network includes myeloid-derived suppressor cells (MDSC), tumor-associated macrophages (TAMS), and dendritic cells (DC). Each of these cell populations has inherent immune suppressive activity which is enhanced through their interactions with each other. These interactions can be either one-way interactions or mutually beneficial interactions (cross-talk) that are often exacerbated by inflammation in the tumor microenvironment. After a brief description of each of these cell populations, this article

\footnotetext{
- Corresponding author at: UMBC, Dept. Biological Sciences, 1000 Hilltop Circle, Baltimore, MD 21250, United States. Tel." +1 410455 2237: fax: +1 4104553375 .

E-mail address: srosenbeøumbc.edu (S. Ostrand-Rosenberg).
}

doi:10.1016/j.semcancer.2012.01.011

will focus on how interactions and cross-talk between the various myeloid cell populations enhance immune suppression and promote tumor growth.

\section{Myeloid-derived suppressor cells (MDSC)}

MDSC are immune suppressive immature myeloid cells that are elevated in virtually all patients and experimental mice with malignancies. MDSC include two major subpopulations of cells: monocytic and granulocytic (polymorphonuclear) MDSC, as defined by their expression of plasma membrane markers and their content of immune suppressive molecules. They enhance tumor growth through both non-immune and immune suppressive mechanisms. Their principle non-immune mechanism is the promotion of angiogenesis [4]. They inhibit both adaptive and innate antitumor immunity through a variety of diverse mechanisms, (i) MDSC inhibit $T$ cell activation and function by producing reactive oxygen and nitrogen species (ROS and RNS) which down-regulate or dissociate the $\mathrm{CD} 3$-associated $\zeta$ chain from the T cell receptor (TcR) [5,6], by disrupting signaling through the IL-2 receptor [7], and by preventing antigen/MHC peptide recognition by nitrating the TCR [8] and MHC class I [9] molecules, (ii) MDSC deplete their environment of arginine [10] and L-cysteine [11], amino acids required for $\mathrm{T}$ cell activation and proliferation, (iii) MDSC disrupt T cell migration to lymph nodes by releasing ADAM 17 which downregulates the homing receptor CD62L. (L-selectin) on T cells [12] and they inhibit intratumoral migration of effector $\mathrm{CD} 8^{+} \mathrm{T}$ cells by peroxynitrite modification of the chemoattractant CCL.2 [13]. (iv) MDSC promote the expansion of natural T regulatory cells and drive the development of induced T regulatory cells through their 
production of IL-10, TGF $\beta$, IFN $\gamma$ and through CD40-CD40L interactions [14-16].

In addition to these mechanisms which directly affect $\mathrm{CD} 4^{+}$ and $\mathrm{CD}^{+} \mathrm{T}$ cells, MDSC also use diverse mechanisms to suppress innate immunity. They inhibit natural killer cell cytotoxicity and inhibit NK cell production of IFN $\gamma$ through a cell-contactdependent mechanism [17-20] that involves membrane-bound TGF $\beta$ [17] or recognition of the NK cell receptors NKp30 [21] or NKG2D [17]. Innate anti-tumor immunity is also impacted by the effects of NKT cells on MDSC. There are two types of NKT cells: NKT I or inducible NKT (iNKT) cells facilitate tumor rejection [22] while NKT II cells promote tumor growth [23]. IL-13 production by NKT II cells increases the accumulation of $\operatorname{MDSC}[23,24]$.

\section{Tumor-associated macrophages}

In healthy individuals macrophages are key cells that promote host survival by regulating adaptive immunity, promoting wound healing, and eliminating infectious agents (reviewed in [25]). Similar to MDSC, macrophages are a diverse population of myeloid cells and facilitate tumor progression via both immunological and nonimmunological mechanisms. They form a continuous spectrum of cells that range in phenotype from M1-like or classically activated macrophages to M2-like or alternatively-activated macrophages [26]. M1-like macrophages are typically activated by IFN $\gamma$ and lipopolysaccharide, and are characterized by their high expression of IL-12 and low expression of IL-10. This cytokine profile promotes the development of a type $1 \mathrm{~T}$ cell response which facilitates anti-tumor immunity. In addition, M1-like macrophages can be tumoricidal, In contrast, M2-like macrophages are activated by IL_-4, IL-13, IL-10, and glucocorticoid hormones, produce high levels of IL-10 and low levels of IL-12, and promote tumor progression. Recent studies have identified up to seven phenotypically distinct macrophage subpopulations within tumors, demonstrating the complexity of this cell population [27]. Macrophage phenotype is driven by their local tissue microenvironment and the tumor microenvironment strongly polarizes macrophages towards an M2-like phenotype, giving rise to so-called "tumor associated macrophages" (TAMs). In addition to their immune suppressive activity. TAMs also promote tumor progression through multiple non-immune mechanisms including facilitating angiogenesis [28], promoting tumor cell invasion and metastasis [29], and protecting tumor cells from chemotherapy-induced apoptosis [30].

\section{Dendritic cells}

The major function of dendritic cells (DC) is to process and present antigen for the activation of $\mathrm{CD}^{+}$and $\mathrm{CD} 8^{+} \mathrm{T}$ cells. Endocytosis of antigen by immature DC drives DC maturation and the subsequent presentation of antigen to T cells. However, the tumor microenvironment systemically perturbs this process by increasing the accumulation of immature DC and decreasing DC maturation [31]. As a result, DC fail to activate tumor-reactive T cells and/or become tolerogenic. Defective dendritic cell function has been found in many patients with a variety of cancers, as well as in most mice with transplanted or spontaneous tumors [31-37]. Multiple conditions and factors within the tumor microenvironment, including hypoxia, lactic acid build-up, and adenosine accumulation, cause DCabnormalities (reviewed in [38]). As discussed below, many of these same conditions also foster cross-talk between the myeloid cell populations.

\section{Bidirectional cross-talk between MDSC and macrophages exacerbates immune suppression}

In individuals with tumors, the accumulation and suppressive activity of MDSC and TAMs is initiated by factors produced by tumor cells. Many of these factors act directly on MDSC and TAMs. However, interactions between MDSC and macrophages further exacerbate suppression by these cells by altering cytokine production and expression of cell surface molecules important for cellular function (Fig. 1).

Tumoricidal M1-like macrophages have a phenotype of IL$12^{\text {hi }}$ IL- $10^{\text {lo }}$ and are activated by LPS and IFN $\gamma$. However, co-culture of LPS and IFN $\gamma$-activated peritoneal macrophages with tumorinduced MDSC for $16 \mathrm{~h}$ down-regulates macrophage production of IL-12 by $>80 \%$ [39]. Similar to MDSC suppression of T cell activation [7,40,41], MDSC-mediated down-regulation of IL-12 requires MDSC-macrophage cell contact. MDSC produce high levels of IL-10 and IL-10 is a key cytokine for regulating IL-12 transcription [42]. Co-cultures of tumor-induced MDSC from IL-10-deficient mice with wild type macrophages confirmed that MDSC production of IL-10 mediated IL-12 down-regulation. Interestingly, macrophages themselves exacerbate their polarization towards an M2 phenotype because they stimulate MDSC to synthesize more IL-10 [39].

Increased MDSC production of IL-10 and decreased macrophage production of IL-12 are also likely to impact $\mathrm{CD} 4^{+} \mathrm{T}$ cells and natural killer (NK) cells, IL-10 drives the differentiation of type $2 \mathrm{CD} 4^{+}$ $\mathrm{T}$ (Th2) cells, while IL-12 induces differentiation of type $1 \mathrm{CD}^{+}$ $\mathrm{T}$ (Th1) cells and NK cells. Increased production of IL-10 and the absence of IL-12 therefore favor the development of Th 2 cells and a decrease in NK cells. Th2 cells counter-act the development of cytotoxic CD $8+$ T cells (CTL). They also produce IL -4 which contributes to the development of tumor-associated macrophages [43]. Because IL-10 is also a potent inducer of CD4 ${ }^{+} \mathrm{T}$ regulatory cells (Tregs) [44], MDSC-produced IL-10 is also likely to facilitate the development of Tregs.

MDSC and macrophage bidirectional cross-talk also alters macrophage expression of MHC class II molecules. Although not as efficient as DC, macrophages are effective antigen presenting cells and cross-prime and cross-present both MHC class I and MHC class II-restricted peptides. Co-culture studies with tumor-induced mouse MDSC and macrophages demonstrated that MDSC significantly reduced macrophage expression of MHC II molecules, MHC II down-regulation required MDSC-macrophage cell-to-cell contact and experiments with IL-10-deficient MDSC indicated that the reduction was mediated by IL-10 produced by MDSC (Clements and Ostrand-Rosenberg, unpublished results). IL-10 may downregulate MHCII by increasing transcription of March 1, a ligase that ubiquitinates the cytoplasmic tail of MHC II molecules in monocytes [45-47]. The net result is that macrophages are less effective antigen presenting cells, further diminishing $T$ cell activation and enhancing immune suppression/tolerance.

Studies with human monocytes/macrophages and $\mathrm{T}$ cells from mice with Letshmanta donovant have demonstrated that IL-10 and IL-12 are at least partially regulated through the phosphatidylinositol-3-kinase (PI3K) - mammaliam target of rapamycin (mTOR) pathway. In these reports, rapamycin, an inhibitor of the mTOR pathway, reduced IL-10 production in macrophages [48] and in Th2 CD4 $4^{+} \mathrm{T}$ cells [49], and increased IL-12 production in Th2 $\mathrm{CD}^{+} \mathrm{T}$ cells [49]. These findings raised the possibility that rapamycin may inhibit macrophageMDSC cross-talk and reduce MDSC production of IL-10 and restore macrophage production of IL-12. MDSC-macrophage cocultures treated with rapamycin produce less IL-10 and more IL-12. However, rapamycin is only effective if both MDSC and macrophages are present, indicating that the drug is not acting directly on MDSC to inhibit IL-10 production or 


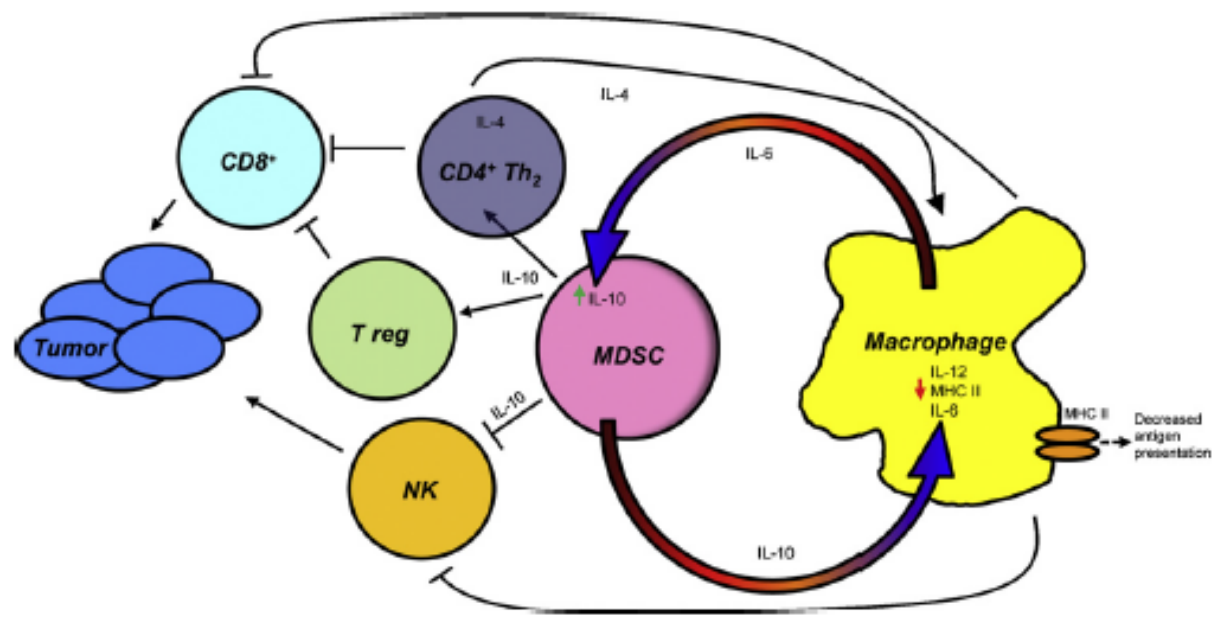

Fig. 1. Cross-talk between MDSC and macrophages polarizes immunity towards a type 2 response that promotes tumor progression. MDSC and macrophages interact with each other through a variety of soluble mediates and cell contact-dependent mechanisms that enhance the suppressive activity of each cell type. Cross-talk results in increased production of IL-10, decreased production of IL-12 and IL-6, and down-regulation of macrophage MHC IL. The down-stream effects are the activation of CDA Th2 and T regulatory cells and decreased antigen presentation which impair cytotoxic CD8+ T cell activity and impairment of NK cell cytotoxicity. Green arrows indicate molecules that are increased; small red arrows indicate molecules that are decreased; large orange/red semi-circular arrows indicate donor to recipient cell effects.

directly on macrophages to promote IL-12 production (Clements and Ostrand-Rosenberg, unpublished results). These findings suggest that rapamycin or other mTOR inhibitors, may be useful therapeutic agents to diminish MDSC-macrophage crosstalk.

The bidirectional nature of MDSC-macrophage interactions significantly amplifies the levels of IL-10 and decreases the levels of IL-12. Therefore, in the tumor microenvironment where both MDSC and macrophage co-exist, IL-10 and IL-12 levels are most likely dramatically increased or decreased, respectively, relative to the cytokine level of either cell population by itself, As a result, MDSC-macrophage bidirectional cross-talk has the potential to further enhance immune suppression.

\section{Inflammation exacerbates bidirectional cross-talk between MDSC and macrophages}

The accumulation of MDSC as well as the immune suppressive mechanisms used by MDSC are exacerbated by chronic inflammation [50-53], and inflammation also increases cross-talk between MDSC and macrophages (Fig. 2). The effect of inflammation on MDSC-macrophage cross-talk was demonstrated using two approaches to increase the inflammatory milieu, In one approach, tumor cells were transfected with the gene encoding IL- $1 \beta$ so the tumor microenvironment contained heightened levels of IL-1 $\beta$ which is upstream of many additional pro-inflammatory mediators, In a second approach MDSC were generated in IL-1 receptor antagonist-deficient (IL-1 $\mathrm{Ra}^{-1-}$ ) mice, In the absence of IL-1Ra, mice cannot attenuate IL-1 $\beta$ signaling and therefore have heightened inflammation. MDSC induced under conditions of high IL-1B ("inflammatory" MDSC) synthesize more IL-10 than MDSC induced in less inflammatory settings ("conventional" MDSC), and the presence of macrophages further increases the production of IL-10 by inflammatory MDSC [54]. This increase in IL-10 is due to macrophage production of IL-6 since co-cultures of MDSC and IL-6-deficient macrophages contain less IL-10 than co-cultures of MDSC and wild type macrophages (Beury and Ostrand-Rosenberg. unpublished results). Since IL-1 $\beta$ is a key regulator of IL-6 [55].
IL-1 3 most likely increases MDSC production of IL-10 by increasing macrophage and MDSC synthesis of IL-6 which in turn increases MDSC production of IL-10.

In addition to IL-1 $\beta$, pro-inflammatory bioactive lipids also increase MDSC-macrophage cross-talk to promote immune suppression, Prostaglandin E2 $\left(\mathrm{PGE}_{2}\right)$, a product of arachidonic acid metabolism, binds to all four prostanoid receptors (EP-1, $-2,-3$, and -4 ) and Butaprost, a PGE 2 analogue that only binds to EP2, both drive the differentiation of MDSC from c-kit' hematopoietic progenitor cells [56]. PGE $_{2}$ and Butaprost also increase MDSC production of IL-10 in the presence of macrophages, In contrast to the effects of IL-1B, this cross-talk-mediated increase in IL-10 does not require MDSC-macrophage cell-to-cell contact, indicating that strictly soluble factors are responsible (Clements and OstrandRosenberg, unpublished data)

At a mechanistic level, the increase in IL-10 is mediated by signaling through MDSC-expressed TLR4 because MDSC from TLR4-deficient mice do not have higher levels of IL-10. Interestingly, macrophage-induced up-regulation of IL-10 by MDSC also requires signaling through TL.R4 on macrophages since TLR4deficient macrophages are unable to increase MDSC production of IL-10,

The MDSC-macrophage cross-talk experiments described above were performed in the presence of lipopolysaccharide (LPS), a known activator of macrophages. Signaling through TLR4 typically involves the binding of LPS to the L.PS binding protein, which subsequently transfers LPS to the membrane-bound receptor CD14. CD14 then complexes with TLR4 to initiate TLR4 signaling and down-stream activation of NFkB [57]. CD14 levels are increased on inflammatory MDSC during cross-talk with macrophages and this increase is TL.R4-dependent because TL.R4-deficient inflammatory MDSC do not display elevated levels of CD14 [54]. Therefore, inflammation most likely increases CD14 levels which may make MDSC more responsive to LPS and other TLR4 activating ligands, thereby driving MDSC production of IL-10 and the resulting in immune suppression.

As a result of their increased production of IL-10, inflammatory MDSC are significantly more efficient at down-regulating 


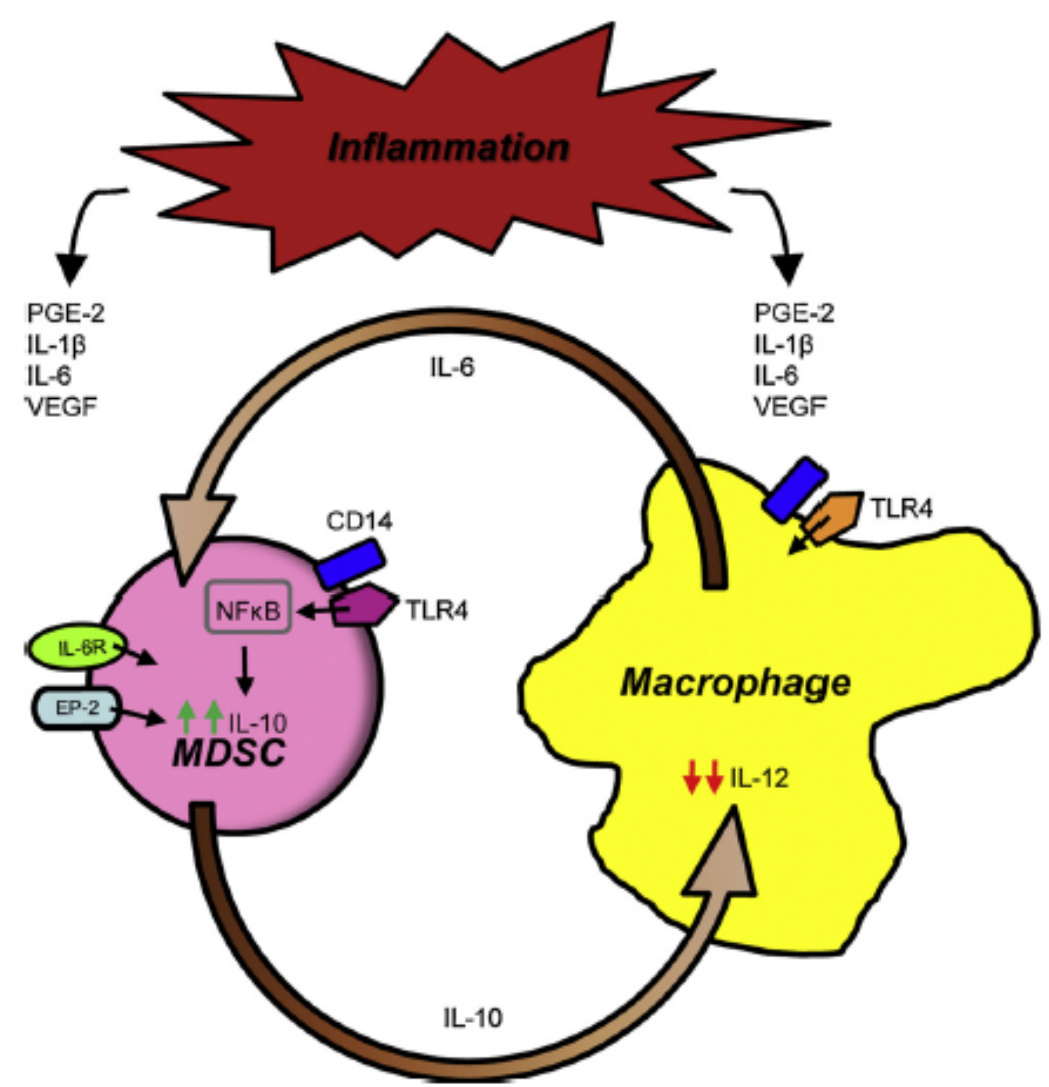

Fig. 2. Inflammation enhances MDSC-macrophage cross-talk. Tumor and stromal cells within the tumor microenvironment secrete a variety of inflammatory mediators. For example, tumar cells produce PGE2 which activates MDSC through the EP receptors and COX2 which promotes the production of PCE2 from arachidonic acid. Tumar cells and MDSC produce VECF, IL-6 and S100A8/A9 proteins which bind to their respective receptors on MDSC, as well as IL-1 $\beta$ which acts via Il-6. These mediators accentuate the cross-talk between MDSC and macrophages and further increase MDSC production of IL-10 and decrease macrophage production of IL-12. Inflammation-increased MDSC production of IL-10 is TLR4-dependent and involves up-regulation of CD14.

macrophage production of IL-12. These findings demonstrate direct role of inflammation in promoting $\mathrm{M} 2$ polarization of macrophages and thereby promoting immune evasion,

\section{Inflammation increases MDSC-NK cell cross-talk}

In addition to their cross-talk with other myeloid cells, MDSC also impact NK cells and reduce their suppressive activity [18]. and inflammation increases these effects in a unidirectional fashion [20]. NK cell differentiation is characterized by the expression of CD27 on immature NK cells and increasing expression of CD1 $1 \mathrm{~b}$ and KLRG-1 as NK cells mature [58]. Inflammation, via IL-1B, decreases the levels of CD27 on immature $\mathrm{CD} 27^{+} \mathrm{NK}$ cells in the bone marrow, and eliminates CD11 $\mathrm{b}^{+} \mathrm{KLRG}-1^{+}$NK cells in the spleen. Inflammation also decreases NK cell expression of the NK cell activating receptor NKG2D, presumably making it more difficult to activate NK cells, and reduces NK cytotoxic activity. These effects are mediated by a Ly6Clow subpopulation of granulocytic MDSC which are preferentially expanded by IL-1 13 [20].

In contrast to the Ly6Clow MDSC population of the previous paragraph, monocytic MDSC have been reported to express the NK activating ligand Rae1 and activate NK cells through the NK receptor NKG2D. NK cells activated by MDSC subsequently kil
MDSC [59]. This report and that of [20] each used only one tumor. Therefore, not enough studies have been completed using different tumor systems to determine if NK cell suppression and activation are characteristic of granulocytic Ly6Clow and monocytic MDSC respectively, or if these findings are limited to the MDSC induced by the particular tumors used in these reports.

\section{MDSC-macrophage cross-talk reduces inflammation}

Within the tumor microenvironment tumor cells and stromal cells, including MDSC and macrophages, generate a proinflammatory environment. Different tumor cells produce a variety of pro-inflammatory mediators including prostaglandins, cyclooxygenases, IL-6, TNF $\alpha$, as well as many other mediators [60]. Although inflammation drives MDSC accumulation and suppressive potency [53], and MDSC themselves produce inflammatory mediators [61,62], MDSC also reduce inflammation through their production of the anti-inflammatory cytokine IL-10, MDSC further decrease inflammation by down-regulating macrophage production of the pro-inflammatory cytokine IL-6. This down-regulation requires MDSC-macrophage cell-to-cell contact, and is enhanced by rapamycin treatment (Beury, Clements, and Ostrand-Rosenberg. 


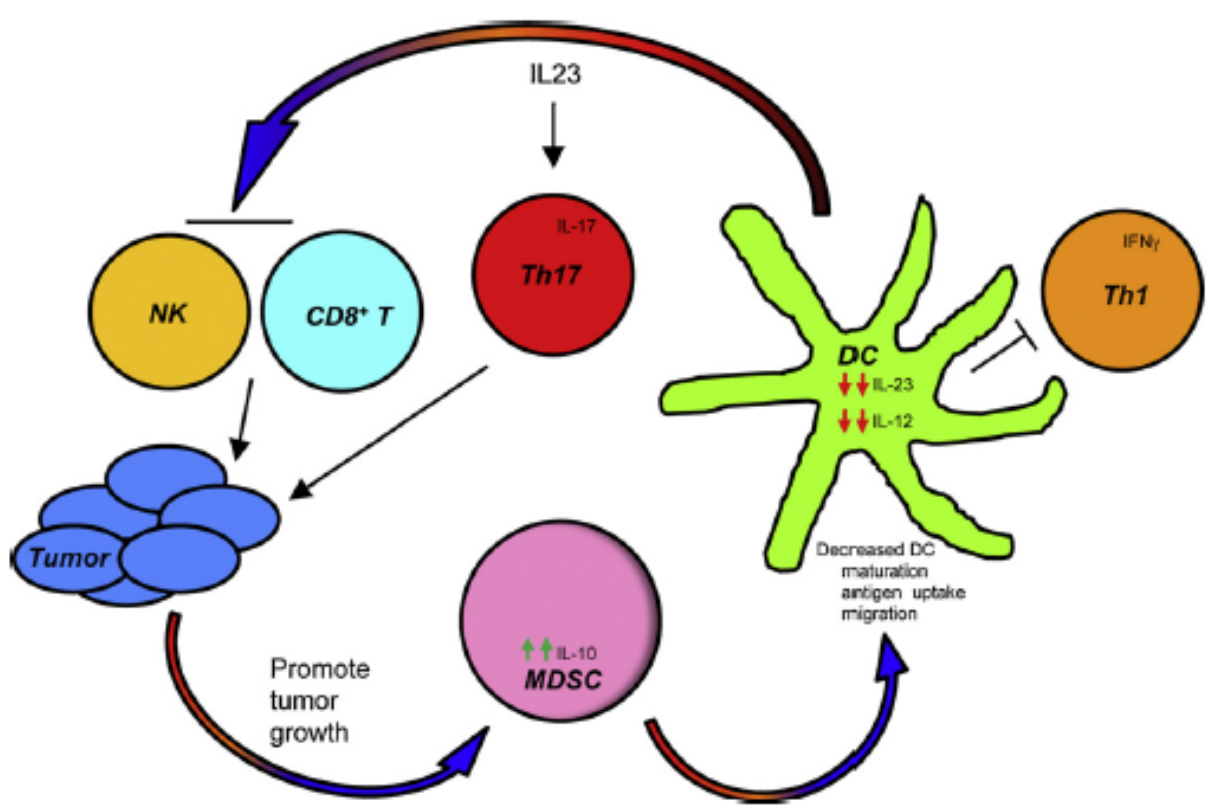

Fig. 3. Cross-talk between MDSC and dendritic cells impairs DC function and promotes tumor progression. Tumor-associated DC produce IL-23 which reduces tumor infiltration of CDS* T cells and suppresses NK cell cytotoxicity, thereby promoting tumor growth. IL-23 also induces Th17 cells that secrete IL-17 which supports tumor progression. Increased tumor burden facilitates the accumulation of MDSC which in turn decrease DC maturation, antigen uptake, migration, IL-23, IL-12 and T cell IFNy production, thereby limiting the activation of CDS-mediated anti-tumor immunity.

unpublished results), consistent with the concept that IL-6 is at least partially regulated by mTOR [63].

The ability of MDSC-macrophage cross-talk to both promote and reduce inflammation may at first appear to be contradictory. However, these dual functions may exist as mechanisms to homeostatically balance the tumor microenvironment. Chronic inflammation is needed to promote the development of MDSC and TAMs; however, acute inflammation activates adaptive T cell immunity with the potential to mediate tumor rejection. By modulating the inflammatory tumor microenvironment, MDSC-macrophage cross-talk reduces the opportunity to activate tumor-reactive $\mathrm{T}$ cells and thereby provides an environment for immune escape and continued tumor progression.

\section{MDSC-DC cross-talk contributes to DC dysfunction}

In contrast to MDSC-macrophage interactions, there is less information on cross-talk between MDSC and DC. As discussed above, in many cancer patients the numbers of mature DC are reduced and DC function is deficient. Although multiple factors are likely to contribute to DC dysfunction, evidence is accumulating that MDSC-DC cross-talk may at least be partially responsible. In vitro studies in which mouse MDSC were differentiated from ckit' bone marrow progenitor cells in the presence of IL-4, GM-CSF, and $\mathrm{PGE}_{2}$ demonstrated that the numbers of mature DC decreased proportionately to the increasing numbers of MDSC [56]. The differentiation of murine DC was similarly reduced when mixtures of murine myeloid cells were treated with LPS and IFN $\gamma$ [64]. IL10 produced by hepatocellular carcinoma-induced MDSC has also been shown to decrease DC production of IL-12 [65]. Since MDSC and DC share a common progenitor cell, the reduction in mature DC observed in cancer patients may be due to the skewing of the common MDSC/DC progenitor towards the preferential differentiation of MDSC at the expense of DC (Fig. 3).

Recent in vitro studies assessing the effects of MDSC from cancer patients on DC differentiation further support a role for MDSC-DC cross-talk. Studies with MDSC from melanoma patients demonstrated that MDSC impaired DC maturation by reducing antigen uptake, preventing migration of immature and mature DC blocking the ability of DC to induce IFN $\gamma$-producing $\mathrm{T}$ cells, and skewing DC cytokine production towards an anti-inflammatory phenotype [66]. DC production of the pro-inflammatory cytokine IL-23 and its downstream induction of Th17 cells may contribute to the effects of MDSC on DC. IL-23 closely resembles IL-12; however, it drives a divergent immunological pathway [67]. Whereas IL-12 induces IFN $\gamma$-producing Th1 cells which mediate cytotoxic responses and tumor rejection, IL-23 promotes tumor progression. IL-23 mediates these effects by driving the proliferation and inflammatory function of Th17 cells [68], which suppress immune surveillance and promote metastasis by impacting adaptive [69] and innate [70] immunity. To determine if MDSC impact IL-17 levels and DC production of IL-23, bone marrow-derived DC from healthy mice were co-cultured with MDSC and the DC subsequently sorted and incubated with transgenic $\mathrm{CD} 4^{+} \mathrm{T}$ cells and cognate peptide. IL-23 (p19/p40) levels were significantly reduced and IL-17 (IL-17A and IL-17AF) levels were modestly reduced in the presence of MDSC-conditioned DC relative to cultures with non-MDSC-conditioned DC (Sinha and Ostrand-Rosenberg. unpublished results). Because IL-23 and IL-17 promote tumor progression, these findings suggest that MDSC may reduce tumor progression by limiting IL-23 and IL-17 production. Therefore, the two studies identify apparently opposing roles for MDSC. These apparent differences could be due to the different species and subpopulations of MDSC used in the two studies since Poschke et al, used monocytic MDSC from melanoma patients 
and peripheral blood-derived human DC, while our study used murine granulocytic MDSC and bone-marrow-derived murine DC. In addition, Poschke et al, did not assess IL-23 or IL-17 levels. However, these findings may also represent the schizophrenic nature of MDSC as seen in their ability to limit tumor progression by activating NK cells [59]. Further studies are needed to clarify the role of MDSC in regulating DC function, and the complexity of the tumor microenvironment with respect to the milieu of cytokines and chemokines is likely to make this task difficult,

\section{Conclusions}

The tumor microenvironment includes diverse host cells that are chemoattracted and induced by tumor-produced factors to generate a highly immune suppressive environment. This review has described some of the host cell cross-talk between MDSC, macrophages, and DC that results in suppressing anti-tumor immunity. Because we are just beginning to understand the complexity of the tumor microenvironment, it is likely there are additional interactions that further promote tumor progression through currently unknown immunological and non-immunological mechanisms. Cross-talk between MDSC, macrophages, and DC promotes synergy amongst these cells and thereby amplifies the immune suppressive effects of the individual cell populations. In addition, induction of one population favors the development of the other populations, and chronic inflammation further increases suppressive potency. As a result, MDSC, macrophages, and DC in the tumor microenvironment are inextricably interconnected such that functions of one population are impacted by the quantity of the other populations. This co-dependency benefits the tumor, but also implies that therapies that down-regulate one population may also reduce the immune suppressive activity of other cell populations. This outcome is most likely applicable to MDSC and macrophages since these two myeloid populations directly impact each other in a reciprocal fashion via their production of IL-10 and IL-6, respectively. Reducing MDSC quantity or function is also likely to increase T cell activation by macrophages since macrophage levels of MHC II will be restored. Antigen presentation by DC is also likely to improve since reduced numbers of MDSC will eliminate the competition between MDSC and DC and promote the expansion and maturation of immunocompetent DC. Whether therapies that uniquely target DC will have down-stream effects on MDSC and macrophages remains unclear, since DC have not as yet been shown to impact MDSC or macrophage development or function. Regardless of whether DC affect MDSC and macrophages, future studies should be aimed at developing therapies that interfere with MDSC-macrophage-DC interactions such that the potent synergistic activity of these cells is neutralized and immune competence can be restored.

\section{Acknowledgements}

The authors thank Ms, Lakshmi Gorrepati for performing the initial IL-23 experiments and Jonathan Weiss for suggesting the rapamycin and mTOR experiments. Original studies were supported by NIH R01CA115880, R01CA84232 (SOR), and American Cancer Society IRG-97-153-07 (PS). DWB is supported by a pre-doctoral fellowship from the DOD Breast Cancer Program (W81XWH-11-1-0115).

\section{References}

[1] Trial watch: ipilimumab success in melanoma provides boost far cancer immunatherapy. Nat Rev Drug Discov 2010;9:584.
[2] Hodi FS, O'Day SJ, McDermatt DF, Weber KW, Sosman JA, Haanen JB, et al. Improved survival with ipilimumab in patients with metastatic melanoma. N Engl J Med 2010:363:711-23.

[3] Brahmer JR, Drake CG, Wollner 1, Powderly JD, Picus ], Sharfman WH, et al.Phase I study of single-agent anti-programmed death-1 (MDX-1106) in refractory solid tumors: safety, clinical activity, pharmacodynamics, and immunologic correlates. J Clin Oncol 2010:28:316 -75.

[4] Yang L, DeBusk LM, Fukuda K, Fingleton B, Green-Jarvis B, Shyr Y, et al. Expansion of myeloid immune suppressor $\mathrm{Gr}+\mathrm{CD} 11 \mathrm{~b}+\mathrm{cellls}$ in tumor-bearing host directly promotes tumar angiogenesis. Cancer Cell 2004:6:409-21.

[5] Ezemitchi AV, Vaknin I, Cohen-Daniel L, Levy O, Manaster E, Halabi A, et al. TCR zeta down-regulation under chronic inflammation is mediated by myeloid suppressar cells differentially distributed between various lymphatic organs. ) Immunol 2006:177:4763-72.

[6] Nagaraj S, Schrum AG, Cho HI, Celis E, Gabrilovich DL. Mechanism of T cell tolerance induced by myeloid-derived suppressor cells. I Immunol 2010:184:3106-16.

[7] Mazzoni A, Bronte V, Visintin A, Spitzer JH, Apolloni E, Seraftni P, et al. Myeloid suppressar lines inhibit $\mathrm{T}$ cell responses by an NO-dependent mechanism. ] 168:689-95.

[8] Nagaraj S, Gupta K, Pisarev V, Kinarsky L, Sherman S, Kang L, et al. Aitered recognition of antigen is a mechanism of CD8 + T cell tolerance in cancer. $\mathrm{Nat}$ Med 2007; $13: 828-35$

[9] LuT, Ramakrishnan R, AltiokS, Youn JL, Cheng P, Celis E, et al. Tumor-inflitrating myeloid cells induce tumor cell resistance to cytotoxic T cells in mice. J Clin Invest 2011:121:4015-29.

[10] Rodriguez PC, Quiceno DC, Zabaleta f, Ortiz B, Zea AH, Piazvelo MB, et al. Arginase 1 production in the tumor microenvironment by mature myeloid cells inhibits T-cell receptor expression and antigen-specinc T-cell responses. Cancer Res 2004:64-5839-49.

[11] Srivastava MK, Sinha P. Clements VK, Rodriguez P, Ostrand-Rosenberg S Myeloid-derived suppressar cells inhibit T-cell activation by depleting cystine and cysteine. Cancer Res 2010:70:68-77.

[12] Hanson EM, Clements VK, Sinha P, Ilkowitch D, Ostrand-Rosenberg S. Myeloidderived suppressor cells down-regulate 1 -selectin expression on $\mathrm{CD} 4+$ and CDS + T cells. J Immunol 2009; 183:937-44.

[13] Molan B, Ugel S, Del Porzo F, Soldani C, Zilio S, Avella D, et al. Chemakine nitration prevents intratumoral inflitration of antigen-speciffc T cells. J Exp nitration prevents intratum
Med 201 1:208:1949-62.

[14] Huang B, Pan PY, Li Q, Sato Al, Levy DE, Bromberg ], et al. Gr-1+CD115+ immature myeloid suppressor cells mediate the development of tumor induced T regulatory cells and T-cell anergy in tumor-bearing host. Cancer Res 2006:66:1123-31

[15] Pan PY, Ma G, Weber KJ, Ozao-Choy J, Wang G, Yin B, et al. Immune stimulatory receptor $\mathrm{CD} 40$ is required for T-Cell suppression and T regulatory cel activation mediated by myeloid-derived suppressor cells in cancer. Cancer Res 2010:70:99-108.

[16] Seranni P, Mgebrot S, Noonan K, Borrello L. Myeloid-derived suppressor cells promote cross-tolerance in B-cell lymphoma by expanding regulatory $\mathrm{T}$ cells. Cancer Res 2008:68:5439-49.

[17] Li H, Han Y, Guo Q, Zhang M, Cao X. Cancer-expanded myeloid-derived suppressor cells induce anergy of NK cells through membrane-bound TGF-beta 1. Jimmunol 2009; 182:240-9.

[18] Liu C, Yu S, Kappes J. Wang J. Grizzle WE, Zinn KR, et al. Expansion of spleen myeloid suppressor cells represses NK cell cytotoxicity in tumor-bearing host. Blood 2007;109:4336-42.

[19] Suzuki E, Kapoor V, Jassar AS, Kaiser LR, Albelda SM. Gemcitabine selectively eliminates splenic $\mathrm{Gr}-1+/ \mathrm{CD} 11 \mathrm{~b}+$ myeloid suppressor cells in tumor-bearing animals and enhances antitumor immune activity. Cin Cancer Res 2005:11:6713-21.

[20] Elkabets M, Ribeiro VS, Dinarello CA, Ostrand-Rosentberg S, Di Santo IP, Apte RN, et al. Il-1beta regulates a novel myeloid-derived suppressor cell subRN, et al. Il-1beta regulates a novel myeloid-derived suppressor cell sub-
set that impars NK cell development and function. Eur J Immunol 2010;40: set that impai $3347-57$.

[21] Hoechst B, Voigtlaender T, Ormandy L, Gamrekelashivili J. Zhao F, Wedemeyer $\mathrm{H}$, et al. Myeloid derived suppressor cells inhibit natural killer cells in patients with hepatocellular carcinoma via the NKp30 receptor. Hepatology 2009:50:799-807

[22] Stewart T], Smyth M], Fernando G], Frazer IH, Leggatt GR. Inhibition of early tumor growth requires J alpha 18-positive (natural killer T) cells. Cancer Res 2003:63:3058-60.

[23] Terabe M, Swann J, Ambrosino E, Sinha P, Takaku S, Hayakawa Y, et al. A nonclassical non-Valpha14jalpha18 CDId-restricted (type iI) NKT cell is sumcient for down-regulation of tumor immunosurveillance.J Exp Med 2005:202: $1627-33$.

[24] Terabe M, Matsui S, Park JM, Mamura M, Noben-Trauth N, Donaldson DD, et al. Transforming growth factor-beta production and myeloid cells are an effector mechanism through which CDid-restricted T cells block cytotoxic $T$ lymphocyte-mediated tumor immunosurveillance: abrogation prevents tumor recurrence. J Exp Med 2003: 198: 1741-52.

[25] Mosser DM, Edwards JP. Exploring the full spectrum of macrophage activation. Mosser DM, Edwards JP. Exploring
Nat Rev Immunol 2008:8:958-69.

[26] Mantovani A, Sica A, Allavena P, Garlanda C, Locati M. Tumor-associated macrophages and the related myeloid-derived suppressor cells as paradigm of the diversity of macrophage activation. Hum Immunol 2009:70: $325-30$ 
[27] Movahedi K, Laoui D, Cysemans C, Baeten M, Stange C, Van den Bossche J, et al. Different tumor microenvironments contain functionally distinct subsets of macrophages derived from Ly6C(high) manocytes. Cancer Res 2010:70-5728-39.

[28] Lin EY, Li JF, Gnatovskiy L, Deng Y, Zhu L, Grzesik DA, et al. Macrophages regulate the angiogenic switch in a mause model of breast cancer. Cancer Res 2005:65:11238-46. [29] Qian B, Deng Y, Im JH, Muschel RJ, Zou Y, Li J., et al. A distinct macrophage
population mediates metastatic breast cancer cell extravasation, establishiment and growth. Pl.oS One 2009:4:e6562.

[30] Zheng Y, Cai Z, Wang S, Zhang X, Qian J. Hong S, et al. Macrophages are an abundant component of myeloma microenvironment and protect myeloma cells from chemotherapy drug-induced apoptosis. Blood 2009:114:3625-8.

[31] Gabrilovich D. Mechanisms and functional signincance of tumour-induced dendritic-cell defects. Nat Rev Immunol 2004:4:941-52

[32] Bellone G, Carbone A, Smirne C, Scirelli T, Burfolino A, Novarino A, et al. Cooperative induction of a tolerogenic dendritic cell phenotype by cytokines secreted ative induction of a tolerogenic dendritic cell phenotype by cy
by pancreatic carcinoma cells. J Immunol 2006:177:3448-60.

[33] Lee BN, Follen M, Rodriquez G, Shen DY, Malpica A, Shearer WT, et al. Deflciencies in myeloid antigen-presenting cells in women with cervical squampus intraepithelial lesions. Cancer 2006:107:999-1007.

[34] Ormandy LA, Farber A, Cantz T, Petrykowska S, Wedemeyer H, Horning M, et al. Direct ex vivo analysis of dendritic cells in patients with hepatocellular

carcinoma. World J Gastroenterol 2006:12:3275-82.
[35] Perrot L, Blanchard D, Freymond N, Isaac S, Guibert B, Pacheco Y, et al. Dendritic cells infltrating human non-small cell lung cancer are blocked at immature stage. J Immunol 2007:178:2763-9.

[36] Pinzon-Charry A, Ho CS, Maxwell T, MoGuckin MA, Schmidt C, Furnival C, et al. Numerical and functional defects of blood dendritic cells in early- and latestage breast cancer. Br J Cancer 2007:97:1251-9.

[37] Pinzon-Charry A, Maxwell T, Lopez JA. Dendritic cell dysfunction in cancer: a mechanism for immunosuppression. Immunat Cell Bial 2005:83:451-61.

[38] Gabrilovich D, Ostrand-Rosenberg S, Bronte V. Coordinated regulation of myeloid cells by tumors. Nat Rev Immunol, in press.

[39] Sinha P, Clements VK, Bunt SK, Albelda SM, Ostrand-Rosenberg S. Cross-talk between myeloid-derived suppressor cells and macrophages subverts tumor immunity toward a type 2 response. J Immunol 2007; 179:977-83.

[40] Kusmartsev S, Nefedova Y, Yoder D, Gabrillwich DL. Antigen-specinc inhibition or CDS $+\mathrm{T}$ cell response by immature myeloid cells in cancer is mediated by reactive oxygen species. J immunol 2004:172-989-99.

[41] Sinha P, Clements VK, Ostrand-Rosenberg S. Reduction of myeloid-derived suppressor cells and induction of M1 macrophages facilitate the rejection of established metastatic disease. J Immunol 2005:174:636-45.

[42] Cao S, Liu L, Chesi M, Bergsagel PL, Ho IC, Donnelly RP, et al. Differential regulation of IL-12 and IL-10 gene expression in macrophages by the basic leucine zipper transcription factor C-Maf flbrosarcoml. J Immunol 2002:169:5715-25.

[43] DeNardo DG garreto J日, Andreu P, Vasquez L, Tawnk D, Kolhatkar N, et al $\mathrm{CD} 4(+) \mathrm{T}$ cells regulate pulmonary metastasis of mammary carcinomas by enhancing protumor properties of macrophages. Cancer Cell 2009:16:91-102.

[44] Murai M, Turovskaya O, Kim G, Madan R, Karp CL. Cheroutre H, et al. Interleukin 10 acts on regulatory $T$ cells to maintain expression of the transcription factor Foxp 3 and suppressive function in mice with colitis. Nat Immuno 2009: 10:1178-84.

[45] Thibodeau J.,Bourgeois-Daigneault MC, Huppe G, Tremblay ], Aumont A, Houde $\mathrm{M}$, et al. Interleukin-10-induced MARCH 1 mediates intracellular sequestration of MHC class II in monocytes. Eur J Immunol 2008; 38: 1225-30.

[46] Shin J5, Ebersold M, Pypaert M, Delamarre L., Hartley A, Mellman L. Surface expression of MHC class II in dendritic cells is controlled by regulated ubiquiexpression of MHC class if in dendritic
tination. Nature 2005:444:115-8.

[47] van Niel G, Wubbolts R, Ten Broeke T, Buschow SI, Ossendorp FA, Melief C], et al Dendritic cells regulate exposure of MHC class II at their plasma membrane by oliguubiquitination. Immunity 2006:25:885-94.

[45] Baker AK, Wang R. Mackman N, Luyendyk IP. Raparmycin enhances LPS induction of tissue factor and tumor necrosis factor-alpha expression in macrophages by reducing II-10 expression. Mol Immunal 2009:46:2249-55.
[49] Ohtani M, Nagai S, Kondo S, Mizuno S, Nakamura K, Tanabe M, et al.Mammalian target of rapamycin and glycogen synthase kinase 3 differentially regulate lipopolysaccharide-induced interleukin- 12 production in dendritic cells. Blood 2008:112:635-43

[50] Bunt SK, Sinha P, Clements VK, Leips f., Ostrand-Rosenberg S. Inflammation induces myeloid-derived suppressor cells that facilitate tumor progression. I immunol 2006:176:284-90.

[51] Bunt SK, Yang L. Sinha P. Clements VK, Leips ], Ostrand-Rosenberg S. Reduced inflammation in the tumor microenvironment delays the accumulation of myeloid-derived suppressor cells and limits tumor progression. Cancer Res 2007:67:10019-26.

[52] Song X, Krelin Y, Dvorkin T, Bjorkdahl O, Segal S, Dinarello CA, et al. CD1 1b+/Gr$1+$ immature myeloid cells mediate suppression of $\mathrm{T}$ cells in mice bearing tumors of 1L-1 beta-secreting cells. J Immunol 2005:175:8200-8.

[53] Ostrand-Rosenberg S, Sinha P. Myeloid-derived suppressor cells: linking inflammation and cancer. J Immunol 2009: 182:4499-505.

[54] Bunt SK, Clements VK, Hanson EM, Sinha P, Ostrand-Rosenberg S. Inflammation enthances myeloid-derived suppressor cell cross-t

[55] Cahill CM, Royers JT. Interleukin (II) I beta induction of IL-6 is mediated by a novel phosphatidylinositol 3-kinase-dependent AKT/kappaB kinase alpha a nothway targeting activator protein-1.J Biol Chem 2008:283:25900-12.

[56] Sinha P, Clements VX, Fulton AM, Ostrand-Rosenberg S. Prostaglandin E2 promotes tumor progression by inducing myeloid-derived suppressar cells. Cancer Res 2007:67:4507-13.

[57] Jiang Q, Akashi S, Miyake K, Petty HR. Lipopolysaccharide induces physical proximity between CD14 and toll-like receptor 4 (TLR4) prior to nuclear translocation of NF-kappa B. J Immunol 2000: 165:3541-4.

[58] Huntington ND, Vosshenrich CA, Di Santo JP. Developmental pathways that CA. Di sin to 2007:7:703-14.

[59] Nausch N, Galani IE, Schlecker E, Cerwenka A. Mononuclear myeloid-derived suppressor cells express RAE-1 and activate natural killer cells. Blood 2008:112:4080-9.

[60] BalkwillF, Charles KA, Mantovani A. Smoldering and polarized inflammation in the initiation and promotion of malignant disease. Cancer Cell 2005:7:211-7.

61] Cheng P, Corzo CA, Luetteke N, Yu B, Nagaraj S, Bui MM, et al. Inhibition of dendritic cell differentiation and accumulation of myeloid-derived suppressor cells in cancer is regulated by $5100 \mathrm{~A} 9$ protein. J Exp Med 2008:205:2235-49.

[62] Sinha P, Okoro C, Foell D, Freeze HH, Ostrand-Rosenberg S, Srikrishna G.
Proinflammatory 5100 proteins regulate the accumulation of myeloid-derived Proinflammatory 5100 proteins regulate the acc

[63] Schaefter V, Arbabi S, Garcia IA, Knoll ML, Cuschieri J, Bulger EM, et al. Role of the mTOR pathway in LPS-activated monocytes: influence of hypertonic saline. J Surg Res 2011:171:769-76.

[64] Greifenberg V, Ribechini E, Rossner S, Lutz MB. Myeloid-derived suppressor Cell activation by combined LPS and IFN-gamma treatment impairs DC development. Eur J Imimunol 2009:39:2865-76.

[65] Hu CE, Gan J, Zhang RD, Cheng YR, Huang G]. Up-regulated myeloid-derived suppressar cell contributes to hepatocellular carcinoma development by impairing dendritic cell function. Scand J Gastroenterol 2011:46:156-64.

[66] Poschke L, Mao Y, Adamson L, Salazar-Onfray F, Masucci G, Kiessling R. Myeloidderived suppressor cells impair the quality of dendritic cell vaccines. Cancer Immunol Immunother 2011.

[67] McKenzie BS, Kastelein RA, Cua D. Understanding the IL-23-1L-17 immune pathway. Trends Immunol 2006:Z7:17-23.

[68] Langrish CL, Chen Y, Blumenschein WM, Mattson J, Basham B, Sedgwick JD, et al. IL-23 drives a pathogenic T cell population that induces autoimmune et al. IL-23 drives a pathogenic T cell popt
inflammation. J Exp Med 2005:201:233-40.

[6] Langowski JL, Zhang X, Wu L, Mattson JD, Chen T, Smith K, et al. II.-23 promotes turnour incidence and growth. Nature 2006;442:461-5.

[70] Teng MW, Andrews DM, Mclaughlin N, von Scheidt B, Ngiow SF, Moller A, et al. IL-23 suppresses innate immune response independently of IL-17A during carcinogenesis and metastasis. Proc Natl Acad Sci U S A 2010:10: $8328-33$. 


\section{Glossary}

ADAM17- A disintegrin and metalloproteinase 17

APC - Antigen presenting cell

ARG1 - Arginase 1

ASC - Alanine-serine-cysteine transporter

Bcl3 - B-cell lymphoma 3-encoded protein

Bv8 - Prokineticin 2

CAT2B - Cationic amino acid transporter 2B

CCL - Chemokine (C-C motif) ligand

CCR - Chemokine (C-C motif) receptor

$\mathrm{CD}-$ Cluster of differentiation

CDDO-Me - Methyl 2-cyano-3,12-dioxooleana-1,9(11)dien-28-oate

CDK4 - Cyclin-dependent kinase 4

C/EBP - CCAAT-enhancer-binding protein

$\mathrm{CHOP}-\mathrm{C} / \mathrm{EBP}$ homologous protein

COX2 - Cyclooxygenase-2

CTL - Cytotoxic T lymphocytes 
CTLA4 - Cytotoxic T-lymphocyte-associated protein 4

CXCL - Chemokine (C-X-C motif) ligand

CXCR - Chemokine (C-X-C motif) receptor

DAMP - Damage-associated molecular pattern molecule

DCFDA - Dichlorofluorescein diacetate

eIF - Eukaryotic initiation factor

ER - Endoplasmic reticulum

ERK - Extracellular signal-regulated kinase

Etv3 - Ets Variant 3

Fas - Fas cell surface death receptor

GCN - General control nonderepressible

GM-CSF - Granulocyte macrophage colony-stimulating factor

Gr1 - Granulocyte marker 1

HDAC1 - Histone deacetylase 1

HIF-1 $\alpha$ - Hypoxia-inducible factor-1 alpha

HEV - High endothelial venules

HMGB1 - High Mobility Group Box protein 1

IDO - Indoleamine 2,3-dioxygenase 
IFN $\gamma$ - Interferon gamma
IL - Interleukin

LAP - Liver-enriched activator protein

LIP - Liver-enriched inhibitory protein

Ly6C - Lymphocyte antigen 6 complex, locus C

Ly6G - Lymphocyte antigen 6 complex, locus G

JAK - Janus kinase

Keap1 - Kelch-like ECH-associated protein 1

Mac - Macrophage

Maf - Musculoaponeurotic fibrosarcoma oncogene homolog

MAPK - Mitogen-activated protein kinases

MCF - Mean channel fluorescence

Neh - Nrf2-ECH homology domain

MIP-1 $\alpha$ - Macrophage inflammatory protein 1 alpha

MIP-2 - Macrophage inflammatory protein -2

MDSC - Myeloid-derived suppressor cells

MHC - Major histocompatibility complex

MMP - Matrix metallopeptidases 
NF- $\kappa \mathrm{B}$ - Nuclear factor kappa-light-chain-enhancer of activated B cells

$\mathrm{NK}$ - Natural killer cells

NKT - Natural killer T cells

$\mathrm{NO}$ - Nitric oxide

NOS2 - Inducible nitric oxide synthase

NOS3 - Endothelial nitric oxide synthase

NOX2 - NADPH oxidase

Nrf2 - Nuclear factor (erythroid-derived 2)-like 2

PD-1 - Programmed cell death 1

PD-L1 - Programed death-ligand 1

PD-L2 - Programed death-ligand 1

$\mathrm{PGE}_{2}-$ Prostaglandin E2

PI3 - phosphatidylinositide 3-kinases

RAG-2 - Recombination activating gene 2

RAGE - Receptor for advanced glycation endproducts

ROS - Reactive oxygen species

SBNO2 - Strawberry notch homolog 2

SHIP-1 - Phosphatidylinositol-3,4,5-trisphosphate 5-phosphatase 1 
SOCS3 - Suppressor of cytokine signaling 3

STAT - Signal transducer and activator of transcription

TAM - Tumor associated macrophage

TAP - Transporter associated with antigen processing

TCCM - Tumor-conditioned cell media

TCR $-\mathrm{T}$ cell receptor

TGF $\beta$ - Transforming growth factor beta

TLR - Toll-Like Receptor

TME - Tumor microenvironment

$\mathrm{TNF} \alpha$ - tumor necrosis factor alpha

Treg - Regulatory T cells

TYK2 - Non-receptor tyrosine-protein kinase

TRAIL-DR5 - Tumor-necrosis factor-related apoptosis-death receptor 5

VEGF - Vascular endothelia growth factor

$\mathrm{x}_{\mathrm{c}}{ }^{-}-$Cystine/glutamate antiporter

xCT - Solute carrier family 7 (anionic amino acid transporter light chain, $\mathrm{x}_{\mathrm{c}}^{-}$system), member 11

Zfp36 - Zinc finger protein 36 homolog 
